\author{
Universidade de São Paulo - USP \\ Escola de Engenharia de São Carlos - EESC \\ Departamento de Engenharia Elétrica
}

\title{
ASPECTOS DE MODELAGEM NUMÉRICA DE TRANSISTORES DE FIOS QUÂNTICOS
}

Rafael Vinicius Tayette da Nobrega

Dissertação de mestrado apresentada à Escola de Engenharia de São Carlos, Departamento de Engenharia Elétrica da Universidade de São Paulo, como parte dos requisitos para obtenção do título de Mestre em Ciências, Programa de Engenharia Elétrica - Área de concentração: Telecomunicações.

Orientador: Prof. Dr. Murilo Araujo Romero

São Carlos 


\section{AUTORIZO A REPRODUÇĂO E DIVULGAÇÄO TOTAL OU PARCIAL DESTE TRABALHO, POR QUALQUER MEIO CONVENCIONAL OU ELETRONNICO, PARA FINS DE ESTUDO E PESQUISA, DESDE QUE CITADA A FONTE.}

Ficha catalográfica preparada pela Seção de Tratamento da Informação do Serviço de Biblioteca - EESC/USP

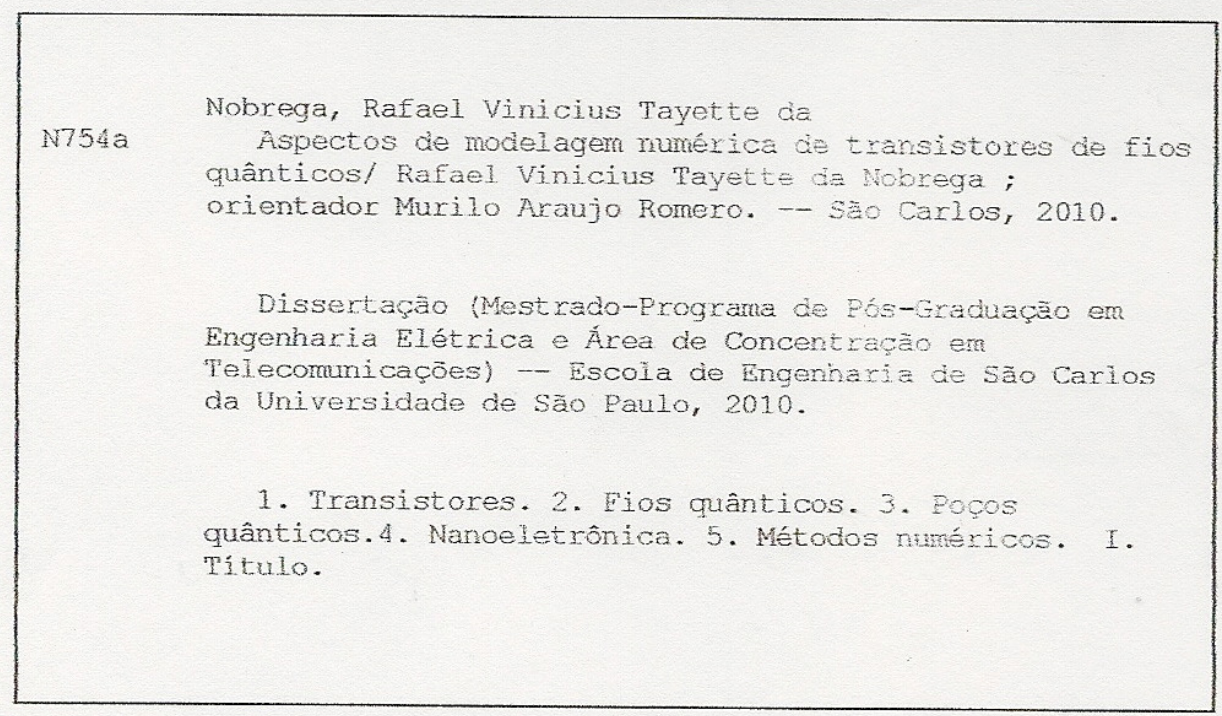




\section{FOLHA DE JULGAMENTO}

Candidato: Bacharel RAFAEL VINICIUS TAYETTE DA NOBREGA.

Dissertação defendida e julgada em 22/07/2010 perante a Comissão Julgadora:

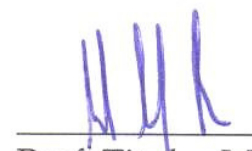

Prof. Titular MURILO ARAUJO ROMERO - (Orientador)

(Escola de Engenharia de São Carlos/USP)

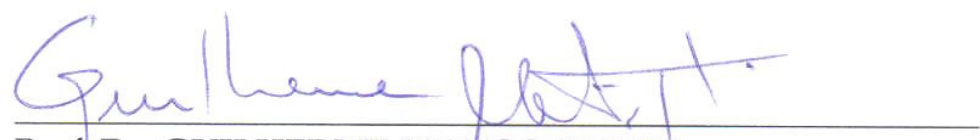

Prof. Dr. GUILHERME MATOS SIPAHI

(Instituto de Física de São Carlos/USP)

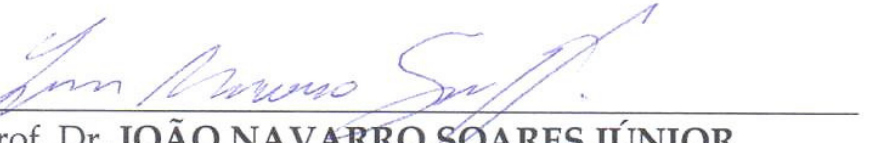

Prof. Dr. JOÃO NAVARRO SOARES JÚNIOR

(Escola de Engenharia de Sáo Carlos/USP)
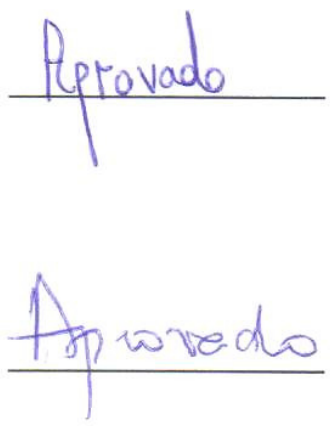

APROVADO

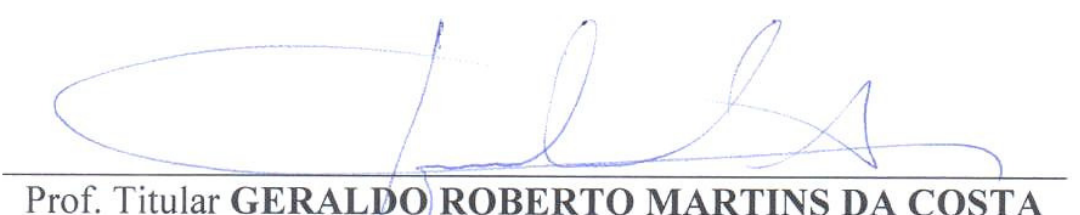

Prof. Titular GERALDO ROBERTO MARTINS DA COSTA

Coordenador do Programa de Pós-Graduação em Engenharia Elétrica e Presidente da Comissão de Pós-Graduação 

"Aprendi que a dureza é a escola adequada para a excelência"

Merlim 



\section{Agradecimentos}

A Deus por estar em minha vida e tornar todos os meus sonhos possíveis e realizáveis.

À FAPESP, Fundação de Amparo à Pesquisa do Estado de São Paulo, pelo fomento a minha pesquisa (Processo N. 2008/52828-4).

Ao meu Orientador Prof. Dr. Murilo Araujo Romero e a minha Co-Orientadora Prof. Dra. Regiane Ragi, pela imensa credibilidade e pela grande orientação. Muito Obrigado!

Agradeço a minha família, em especialmente meu Pai, minha Mãe e minha Irmã por todo o apoio e a confiança depositada em mim desde o começo, tornando possível este sonho. Agradeço também a minha Vó pela a sustentação na fé, tornando toda a minha jornada bem mais fácil. Agradeço, principalmente, minha namorada Mariana que desde sempre acreditou em mim e me ajudou de diversas formas, viabilizando assim a conquista de mais este degrau em minha carreira acadêmica. Amo todos vocês!

A minha família sancarlense, companheiros da República Zero-KCal: Ulysses, Pumba, Zoio, Lixo, Zini, Boy e H.

Aos amigos da Telecom-EESC/USP: Ulysses, Getúlio, Anderson, Alex, Pedro, Daniel, Helvécio, Lorena e Mariana.

A todos os meus colegas de turma de Física-04 da UFSCar.

A todos que direta ou indiretamente colaboraram de uma forma ou de outra com a concretização deste sonho. Peço perdão àqueles que omiti. 



\section{Resumo}

Esta dissertação discute o desenvolvimento de modelos analíticos e numéricos para as características elétricas de transistores de fios quânticos. Sendo assim, realizou-se um estudo implementando uma sequência de formalismos e ferramentas computacionais para solução autoconsistente das equações de Schrödinger e Poisson para poços e fios quânticos. Com a utilização deste método numérico pode-se determinar os auto-estados, os níveis de energias e as densidades eletrônicas de portadores livres, dentre outros parâmetros relevantes para dispositivos de fio quântico.

Adicionalmente, realizou-se um estudo analítico das heteroestruturas semicondutoras de interesse para a área de dispositivos de dimensionalidade reduzida. Este estudo levou a obtenção de resultados referentes ao desenvolvimento de modelos teóricos para as características elétricas de dispositivos baseados no mecanismo de tunelamento ressonante. Os resultados obtidos para a característica corrente-tensão (I-V) nas heteroestruturas investigadas foram contrastados satisfatoriamente com os encontrados na literatura. Este ferramental analítico foi então aplicado para computar o coeficiente de transmissão eletrônico de um diodo de fio quântico com tunelamento ressonante.

Palavras-chave: transistores, fios quânticos, poços quânticos, nanoeletrônica, métodos numéricos. 


\begin{abstract}
This dissertation discusses the development of analytical and numerical models for the electrical characteristics of quantum wire transistors. A study is carried out, implementing a sequence of formalisms and computational tools for the self-consistent solution of the equations of Schrödinger and Poisson in quantum wells and quantum wires. By using this numerical formulation it is possible to determine the eigenstates, energy levels and free-carrier electronic density, among other relevant parameters for quantum wire devices.

In addition, we also conducted an analytical study concerning semiconductor heetrostrucures of interest for reduced dimensionality devices applications. This study led to results regarding the development of theoretical models for the electrical characteristics of devices based on the resonant tunneling mechanism. The results obtained for the current-voltage (I-V) characteristics in the investigated heterostructures were satisfactorily compared to those available at the published literature and this analytical tool was then used to compute the electronic transmission coefficient in a resonant tunneling quantum wire diode.
\end{abstract}

Keywords: transistors, quantum wires, quantum wells, nanoelectronics, numerical methods. 


\section{Sumário}

Lista de Figuras $\quad$ vii

Lista de Tabelas $\quad$ xiii

1 Introdução 1

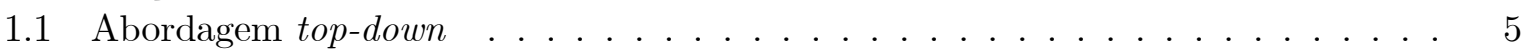

1.2 Abordagem bottom-up . . . . . . . . . . . . . . . . . . . 12

2 Solução auto-consistente das equações de Schrödinger-Poisson para sistemas de fios quânticos $\quad \mathbf{1 6}$

2.1 Solução de sistemas envolvendo poços quânticos . . . . . . . . . . . . . . . . 16

2.1.1 Condições de contorno . . . . . . . . . . . . . . . . . . . . . . . 19

2.1.2 Método Split-Operator . . . . . . . . . . . . . . . . . . . . . . . 21

2.1.3 Estruturas de poços quânticos investigadas . . . . . . . . . . . . . 25

2.2 Solução de sistemas envolvendo fios quânticos . . . . . . . . . . . . . . . . 28

2.2.1 Desenvolvimento do cálculo da densidade eletrônica de $\mathrm{n}(\mathrm{x}, \mathrm{y})$. . . . . . . 32

2.2.2 Solução da Equação de Poisson não-linear pelo Método de Relaxação . . . 33

2.2 .3 Estruturas de fios quânticos investigadas . . . . . . . . . . . . . . . 34

3 Análise do Transporte Eletrônico pelo Formalismo da Matriz Transferência 44

3.1 Fórmula de densidade de corrente de Tsu e Esaki . . . . . . . . . . . . . . . . . . 44

3.1.1 Densidade de corrente J no limite de baixas temperaturas . . . . . . . . . 50

3.2 Cálculo do coeficiente de transmissão pelo Método da Matriz Transferência para

múltiplas barreiras de potencial com e sem polarização aplicada . . . . . . . . . . 53

3.2.1 Uma barreira de potencial com polarização externa aplicada . . . . . . . . . 53

3.2.2 Generalização para múltiplas barreiras de potencial . . . . . . . . . . . . . 69

3.2.3 Resultados de múltiplas barreiras com polarização externa aplicada . . . . 71

3.2.4 Resultados de múltiplas barreiras sem polarização externa aplicada . . . . 76

3.3 Cálculo da densidade de corrente para heteroestruturas de duas e três barreiras . 83

3.4 Diodo de fio quântico com tunelamento ressonante . . . . . . . . . . . . . 86

3.4.1 Cálculo do $\mathrm{T}(\mathrm{E})$ para o diodo de tunelamento ressonante . . . . . . . . . 91

4 Conclusão $\quad 95$

$\begin{array}{ll}\text { Referências Bibliográficas } & 97\end{array}$

A Métodos de Diferenças Finitas Não-Uniforme (MDFNU) 105 
B Fundamentos de mecânica quântica para solução de poços e barreiras de potencial 


\section{Lista de Figuras}

1.1 Diagrama esquemático de uma super-rede de dopagem modulada mostrando as camadas em que os elétrons ficam confinados, o gás de elétrons bidimensional (2D).

1.2 Geometria do dispositivo apresentando uma estrutura de "split-gate" sobre uma heteroestrutura convencional de AlGaAs/GaAs, em que os eletrodos de contato são produzidos, e pelos quais pode-se polarizar o dispositivo com relação ao substrato. Pode-se imaginar uma situação, como ilustrada à direita, em que a tensão aplicada aos contatos, $V_{c}$ remova por completo o canal 2-DEG, abaixo do contato metálico, podendo em seguida variar a largura do fio além da distância $d$. . . . .

1.3 Nesta estrutura a corrosão provocada sobre a superfície da heteroestrutura é capaz de provocar o confinamento eletrônico, dando origem a um fio quântico. . . . . . .

1.4 Seção transversal de um HEMT de fio quântico, proposto por Islam et al na Ref.

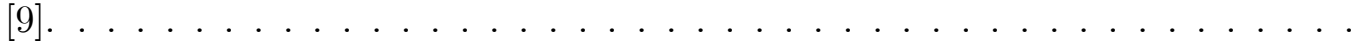

1.5 Esquema da seção transversal de um transistor de tunelamento ressonante de efeito de campo de duplo poço quântico (fora de escala). A área compreendida pela linha seccionada amarela representa o implante de $\mathrm{n}^{+}-\mathrm{Si}$. O tunelamento é representado pela seta dourada, enquanto que a flecha verde representa o caminho da corrente no poço quântico (QW) de GaAs. Dispositivo proposto por Yang et al na Ref. $[18] . \ldots \ldots \ldots \ldots \ldots \ldots . . \ldots \ldots$

1.6 Representação esquemática dos passos de fabricação dos transistores de tunelamento ressonante de nanocoluna. a) crescimento das camadas via MBE, metalização e litografia de feixe eletrônico. b) Definição da nanocoluna por "etching". c) Planarização com várias camadas de HSQ (hydrogen silsesquioxan). d) Etching de plasma isolando a camada de HSQ e litografia de feixe eletrônico para formar o gate. e) Segunda planarização com várias camadas de HSQ. f) Exposição dos contatos do topo da nanocoluna e o gate. g) Esquema das heteroestruturas com uma dupla barreira (AlAs), um poço quântico (GaAs) e regiões de contatos. Esquemas retirados do trabalho de Wensorra, Ref. [27]. . . . . . . . . . . . . 10

1.7 a) Visão esquemática do transistor de tunelamento ressonante de nanocoluna. Micrografias SEM do RTT de nanocoluna: b) vista de fora e c) vista de cima. Micrografias e esquemas retirados do trabalho de Wensorra, Ref. [27]. . . . . . . 11 
1.8 Ilustração esquemática do mecanismo VLS (vapor-liquide-solid) de crescimento de nanofios de InAs. O crescimento do cristal é ilustrado basicamente em dois passos: o primeiro passo envolve o sistema vapor-liquído, no qual a transferência de material da fase de vapor ocorre diretamente na gota de $\mathrm{Au}$ em fase líquida, com a finalidade de supersaturar a gota líquida com o material do cristal de InAs. Já o segundo passo ocorre no sistema líquido-sólido através da precipitação do material contido na liga de $\mathrm{Au}$, que atua como um catalisador e um guia para o crescimento do material na interface líquido-sólido. Esquema retirado do livro de M. Meyyappan e M. K. Sunkara, Ref. [32]. . . . . . . . . . . . . . . . .

1.9 Síntese de um fio quântico de heteroestruturas, onde o círculo dourado representa a liga de ouro, conhecida como catalisador, a) crescimento de um fio quântico semicondutor homogêneo de InAs; b) crescimento de um segundo fio quântico semicondutor de InP; c) repetição dos passos anteriores para a criação de um fio quântico de heteroestruturas semicondutoras. . . . . . . . . . . . . .

1.10 Exemplos de dispositivos construídos por técnicas de fabricação dentro da abordagem "bottom-up": a) Microscopia eletrônica mostrando um fio quântico de InAs com duas barreiras de InP, igualmente espaçadas, retirado do trabalho de Thelander et al, na Ref. [31].b) Esquema de um transistor de fio quântico planar com heteroestrutura, onde uma barreira de $50 \mathrm{~nm}$ de InAsP foi introduzida no fio quântico de InAs, retirado do trabalho de Wernesson et al, na Ref. [34]. . . .

1.11 a) Micrografia eletrônica de nanoestruturas de GaAs, [35], fabricados por técnicas pertencentes a abordagem "top-down". b) Micrografia eletrônica de fios quânticos de InAs, [34], fabricado por técnicas pertencentes a abordagem "bottom-up". . .

2.1 Representação esquemática de um contato Schottky e uma sequência de camadas semicondutoras, que compõem um HEMT, para exemplificar o estudo das condições de contorno. À esquerda tem-se a estrutura de camadas, e à direita, o perfil de potencial para esta estrutura. Nela é possível ver a formação de um canal condutor de elétrons entre as camadas de AlGaAs e GaAs. . . . . . . . . .

2.2 Fluxograma representando o procedimento numérico empregado para a solução do problema auto-consistente. . . . . . . . . . . . . . . .

2.3 Diagrama esquemático da estrutura de camadas da heteroestrutura semicondutora de dopagem modulada $\mathrm{GaAs} / \mathrm{Al}_{0,3} \mathrm{Ga}_{0,7} \mathrm{As} . \ldots \ldots \ldots \ldots$. . . . . . . .

2.4 Perfil de potencial de condução em eV em função da posição na heteroestrutura GaAs $/ \mathrm{Al}_{0,3} \mathrm{Ga}_{0,7} \mathrm{As}$, onde a linha vermelha representa o trabalho desenvolvido e a linha preta o trabalho proposto por Tan et al, na Ref. [47]. . . . . . . . . . .

2.5 População eletrônica por unidade de volume em função da posição na heteroestrutura de GaAs $/ \mathrm{Al}_{0,3} \mathrm{Ga}_{0,7} \mathrm{As}$, proposta por Tan et al, na Ref. [47]. . . . . . . . . .

2.6 Diagrama esquemático da estrutura de camadas das heteroestrutura semicondutoras que constituem o HEMT. . . . . . . . . . . . . . . . . .

2.7 Perfil de banda de condução em meV em função da distância à porta, para as tensões: a) $V_{g s}=-0,5 \mathrm{~V}$ e b) $V_{g s}=-1,5 \mathrm{~V}$, à uma temperatura de $300 \mathrm{~K}$, onde a linha vermelha representa o trabalho desenvolvido aqui e a linha preta representa o trabalho de Vinter na Ref. [69]. . . . . . . . . . . . . . . . . . . .

2.8 População eletrônica por unidade de volume em função da posição em relação à porta, para as tensões: a) $V_{g s}=-0,5 V$ e b) $V_{g s}=-1,5 V \ldots \ldots \ldots$ 
2.9 Diagrama esquemático de transistores de fios quânticos sobre uma heteroestrutura convencional AlGaAs/GaAs com uma estrutura: a) de split-gate e b) corrosão provocando o confinamento eletrônico.

2.10 Representação esquemática da região de solução do cálculo auto-consistente das equações de Schrödinger e de Poisson. Na figura vêem-se contatos que podem polarizar a estrutura. A equação de Schrödinger e de Poisson é resolvida no interior da região marcada pelo retângulo tracejado, que estabelece também as condições de contorno, como o dispositivo mostrado na Fig. (2.9-a). . . . . . . .

2.11 Representação esquemática da região de solução do cálculo auto-consistente das equações de Schrödinger e de Poisson, para estruturas como a da Figura (2.9-b).

2.12 Representação esquemática da estrutura de camadas semicondutoras representando uma mesa rasa, "shallow mesa", a qual é capaz de produzir um confinamento eletrostático lateral, devido ao "etching". A figura é retirada da Referência [13].

2.13 À esquerda, o perfil da banda de condução para o sistema apresentado na Fig.(2.12), e à direita a concentração de elétrons na região do fio quântico, ambos ao longo da direção x. . . . . . . . . . . . . . . . . . . . . .

2.14 À esquerda pode-se observar o perfil de potencial ao longo da direção y, indicada por Snider pela letra x, na Ref. [13] para uma distância de 1500 Å. À direita, está mostrado o resultado obtido a partir da formulação apresentada na Seção (2.2). .

2.15 Geometria do dispositivo proposto na Ref. [72].

2.16 À esquerda, perfil de potencial na temperatura de $4,2 \mathrm{~K}$, próximo à interface de AlGaAs obtida na Ref. [72]. À direita, os resultados obtidos a partir das simulações do método proposto na Seção (2.2).

2.17 À esquerda é mostrado o resultado obtido por Laux et al [72] para a densidade eletrônica no canal unidimensional; à direita é mostrado um resultado obtido a partir da formulação proposta neste trabalho, devendo ser salientado que muitos parâmetros não são totalmente conhecidos para confrontar diretamente os resultados. Ainda assim, pode-se dizer que as simulações obtidas são satisfatórias. . .

2.18 Geometria do dispositivo usado para se obter a relação entre a tensão Vc aplicada aos eletrodos de contato e a concentração eletrônica na interface AlGaAs/GaAs.

2.19 Perfil de potencial lateral, à esquerda, e concentração eletrônica na interface AlGaAs/GaAs em função da tensão $V_{C}$ aplicada aos eletrodos do dispositivo. . . .

2.20 À esquerda é mostrado a densidade eletrônica por unidade de comprimento do fio $\left(\mathrm{n}_{s}\right)$ em função da tensão $V_{c}$ aplicada, e à direita, tem-se a tensão de pico alcançada para cada $V c \ldots \ldots \ldots \ldots \ldots \ldots \ldots$

2.21 a) Um diagrama esquemático de um transistor de fio quântico; b) resultado para a densidade eletrônica controlada por uma tensão de gate, $V_{g}$ para um $V_{c}$ fixo.

3.1 a) Perfil de potencial sem a aplicação de polarização. b) Perfil de potencial com uma tensão aplicada $V \ldots \ldots \ldots \ldots \ldots \ldots$. . . . . . . . . . . . 46

3.2 Perfil de potencial de uma barreira de potencial com polarização aplicada. . . . . 54

3.3 Perfil de potencial de uma barreira de potencial finito com polarização aplicada, utilizando as divisões da barreira inclinada, método denominado "Step Approximation", Ref. [79]. . . . . . . . . . . . . . . . . . . .

3.4 Logaritmo do coeficiente de transmissão em função da tensão aplicada em função do número $N$ de retângulos, usando Método dos Retângulos, para $E=0,05 \mathrm{eV}$, de acordo com a Ref. [54]. . . . . . . . . . . . . . . . . . . 
3.5 Esquema de uma barreira de potencial com polarização aplicada: a) representa uma visualização ampliada da região com a inclinação do potencial, região, da barreira; b) região paralela ao eixo x. . . . . . . . . . . . . . . . .

3.6 Esquema de uma barreira de potencial com polarização aplicada, divida em $N$ retângulos, evidenciando os comprimentos: $L_{1}, L_{2}$ e $L_{3}$. . . . . . . . . . . . . . 69

3.7 Representação de $n$-barreira de potencial com polarização aplicada. . . . . . . 70

3.8 Representação de $n$-barreira de potencial sem polarização aplicada. . . . . . . . . 71

3.9 Logaritmo do coeficiente de transmissão em função da tensão aplicada de uma barreira de potencial unidimensional. Na figura a linha preta, representa o resultado obtido neste trabalho para uma única barreira com polarização aplicada e a linha vermelha representa a Ref. [54]. . . . . . . . . . . . . . . . .

3.10 Representação de uma dupla bareira de potencial com polarização aplicada. . . .

3.11 Coeficiente de transmissão em função da energia de uma dupla barreira: a) Tensão aplicada: 0,16V. b) Tensão aplicada: 0,4V. Em ambas as figuras: a linha preta, representa o nosso resultado para uma dupla barreira com polarização aplicada e a linha vermelha representa a Ref. [55]. . . . . . . . . . . . . . . . . .

3.12 Representação de uma tripla barreira de potencial com polarização aplicada. . .

3.13 Logaritmo do coeficiente de transmissão em função da energia de uma tripla barreira: a) Tensão aplicada de: 0,16V. b) Tensão aplicada de: 0,4V. Em ambas as figuras: a linha preta, representa o nosso resultado para uma tripla barreira com polarização aplicada e a linha vermelha representa a Ref. [55]. . . . . . . . .

3.14 Coeficiente de transmissão em função da energia de uma quadrúpla barreira, para uma tensão aplicada de $0,3 \mathrm{~V}$. A linha preta representa o resultado deste trabalho para o caso da quadrupla barreira de potencial com polarização aplicada e a linha vermelha representa o resultado da Ref. [55]. . . . . . . . . . . . . . . .

3.15 Representação da barreira de potencial finito de comprimento $x_{2}-x_{1}$ e altura $V_{0} .77$

3.16 Coeficiente de transmissão através de uma barreira de potencial em função da energia da partícula incidente na barreira com o comprimento de $20 \AA$ e potencial de $0,347 \mathrm{eV}$, onde a linha preta representa o resultado do formalismo desenvolvido na Seção (3.2.4) e a linha vermelha representa o trabalho proposto por Brennan na Ref. [48]. . . . . . . . . . . . . . . . . . . . .

3.17 Coeficiente de transmissão através de uma barreira de potencial em função da energia da partícula incidente na barreira com o comprimento de $10 \AA$ e potencial de 1,0 eV, onde a linha preta representa o resultado do formalismo desenvolvido na Seção (3.2.4) e a linha vermelha representa o trabalho proposto por Brennan na Ref. [48]. . . . . . . . . . . . . . . . . . . . . . .

3.18 Representação do problema da barreira dupla de potencial finito, de altura $V_{0}$.

3.19 Coeficiente de transmissão através de uma dupla barreira de potencial em função da energia da partícula incidente. As barreiras apresentam comprimento de $50 \AA$ e altura $0,3 \mathrm{eV}$, e o poço quântico um comprimento de $60 \AA$. A linha em preto representa o resultado obtido através do formalismo desenvolvido na Seção (3.2.4) e a linha em vermelho representa o resultado obtido por Dresselhaus na Ref. [54].

3.20 Coeficiente de transmissão através de uma dupla barreira de potencial em função da energia da partícula incidente. As barreiras apresentam comprimento de $20 \AA$ e altura $0,5 \mathrm{eV}$, e o poço quântico um comprimento de 50 Å. A linha em preto representa o resultado obtido através do formalismo desenvolvido na Seção (3.2.4) e a linha em vermelho representa o resultado obtido por Tsu e Esaki na Ref. [53]. 
3.22 Coeficiente de transmissão através de uma tripla barreira de potencial em função da energia da partícula incidente. As barreiras apresentam comprimento de $20 \AA$ e altura $0,5 \mathrm{eV}$, e o poço quântico um comprimento de $50 \AA$. A linha em preto representa o resultado obtido através do formalismo desenvolvido na Seção (3.2.4) e a linha em vermelho representa o resultado obtido por Vatannia na Ref. [55]. .

3.23 Coeficiente de transmissão através de uma tripla barreira de potencial em função da energia da partícula incidente. As barreiras apresentam comprimento de $20 \AA$ e altura $0,5 \mathrm{eV}$, e o poço quântico um comprimento de $50 \AA$. A linha em preto representa o resultado obtido através do formalismo desenvolvido na Seção (3.2.4) e a linha em vermelho representa o resultado obtido por Tsu e Esaki na Ref. [53].

3.24 Coeficiente de transmissão através de uma quíntupla barreira de potencial em função da energia da partícula incidente. As barreiras apresentam comprimento de $20 \AA$ e altura $0,5 \mathrm{eV}$, e o poço quântico um comprimento de $50 \AA$. A linha em preto representa o resultado obtido através do formalismo desenvolvido na Seção (3.2.4) e a linha em vermelho representa o resultado obtido por Tsu e Esaki na Ref. [53].

3.25 Perfil de potencial de uma heteroestutura semicondutora de duas e três barreiras, no qual as barreiras tem $20 \AA$ e os poços $50 \AA$, e a altura da barreira $0,5 \mathrm{eV}$, de acordo com a Ref. [53]. . . . . . . . . . . . . . . . .

3.26 Densidade de corrente de tunelamento em função da tensão aplicada para a heteroestrutura semicondutora de duas barreiras, com a largura da barreira de 20 $\AA$ e altura $0,5 \mathrm{eV}$ e a largura do poço de $50 \AA$, no qual considerou-se a energia incidente do elétron com o valor de $0,005 \mathrm{eV}$. A linha preta representa o resultado obtido neste trabalho e a linha vermelha representa o trabalho proposto por Forrest e Meijer na Ref. [56].

3.27 Densidade de corrente de tunelamento em função da tensão aplicada para a heteroestrutura semicondutora de três barreiras, com a largura da barreira de $20 \AA$ e altura $0,5 \mathrm{eV}$ e a largura do poço de $50 \AA$, no qual considerou-se a energia incidente do elétron com o valor de $0,005 \mathrm{eV}$. A linha preta representa o resultado obtido neste trabalho e a linha vermelha representa o trabalho proposto por Forrest e Meijer na Ref. [56]. . . . . . . . . . . . . . . . . . .

3.28 a) Representação esquemática do diodo de tunelamento ressonante de fio quântico.

b) Representação da dupla barreira de potencial formada a partir das heteroestruturas inseridas no diodo de fio quântico, em função da distância para os casos: sem polarização aplicada e c) com polarização aplicada. . . . . . . . . . . . .

3.29 Coeficiente de transmissão eletrônico do diodo de fio quântico com tunelamento ressonante em função do raio do fio quântico. . . . . . . . . . . . . . . . .

A.1 Representação esquemática da discretização não-uniforme na direção $x . \ldots .106$

B.1 Representação do poço retangular de potencial finito simétrico de comprimento $a$ e altura $V_{0}$, dividido em três regiões: Região 1,2 e $3 . \ldots \ldots \ldots 111$

B.2 Representação das funções de ondas $(\Psi(x))$ para $n=1,2$ e $3 \ldots \ldots$. . . . . 115

B.3 Representação das densidades de probabilidade $\left(|\Psi(x)|^{2}\right)$ para $n=1,2$ e $3 . \quad$. . 116

B.4 Esquema das bandas de energia para um sistema de heteroestrutura semicondutora mostrando os efeitos da quantização espacial e a energia de um fóton emitido após uma recombinação elétron-lacuna. . . . . . . . . . . . . . . . . . . . 119 
B.5 Poço quadrado de potencial finito assimétrico de comprimento L, dividido em três regiões: Região 1,2 e $3 . \ldots \ldots \ldots$. . . . . . . . . . . . . 120

B.6 Esquema do estado acessível do poço de potencial finito assimétrico. . . . . . . . 122

B.7 Representação do poço de potencial triangular, dividido em duas regiões: Região

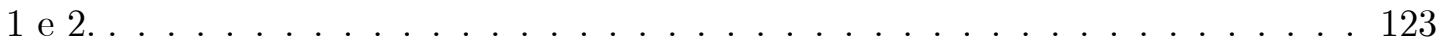

B.8 Comportamento das duas funções de Airy: $A i(x)$ e $B i(x) \ldots \ldots \ldots \ldots$ 


\section{Lista de Tabelas}

2.1 Parâmetros usados no dispositivo estudado por Vinter ～. . . . . . . . . . . . 29

3.1 Parâmetros usados para obtenção da densidade de corrente J para as estruturas mostradas na Fig. (2.34) e $(2.35) \ldots \ldots$. . . . . . . . . . . . 85

3.2 Tunelamento eletrônico em função do raio $\mathrm{R}$ do fio quântico de InAs com barreiras de InP. . . . . . . . . . . . . . . . . . . . . . . . . . . 93 


\section{Lista de Símbolos}

$\begin{array}{ll}V_{C} & \text { tensão no contato } \\ n(x) & \text { densidade eletrônica } \\ E_{k} & \text { energia na } k \text {-ésima sub-banda } \\ J & \text { densidade de corrente } \\ \mathbf{T}(E, V) & \text { coeficiente de transmissão eletrônico } \\ \hbar & \text { constante de Planck dividido por } 2 \pi \\ q & \text { carga elementar do elétron } \\ a(x) & \text { parâmetro de rede } \\ m^{*}(x) & \text { massa efetiva } \\ V_{e f}(x) & \text { potencial efetivo } \\ V(x) & \text { potencial de Hartree } \\ V_{x c}(x) & \text { potencial de correlação e troca } \\ a_{0}^{*} & \text { raio de Bohr efetivo } \\ \varepsilon(x) & \text { permeabilidade elétrica } \\ C_{1} & \text { potencial de deformação na banda de condução } \\ \epsilon_{x x}, \epsilon_{y y} \text { e } \epsilon_{z z} & \text { componentes de strain } \\ \kappa(x) & \text { constante dielétrica do semicondutor } \\ \rho(x) & \text { densidade de carga } \\ N_{d}^{+}(x) & \text { densidade de doadores ionizados } \\ N_{d}(x) & \text { densidade de impurezas doadores } \\ g & \text { degenerescência do nível doador } \\ E_{d} & \text { energia do nível doador } \\ E_{F} & \text { energia do nível de Fermi } \\ g(E) & \text { densidade de estados do sistema } \\ f(E) & \text { func̃o distribuição de Fermi-Dirac } \\ N_{a}^{-}(x) & (x)\end{array}$




\begin{tabular}{|c|c|}
\hline$\Psi(x)$ & funções de onda \\
\hline$q \phi_{B}$ & altura da barreira de potencial \\
\hline$E_{C}$ & banda de condução \\
\hline$E_{V}$ & banda de valência \\
\hline$p_{0}$ & concentração intrínseca de lacunas na banda de valência \\
\hline$K_{B}$ & constante de Boltzmann \\
\hline$T$ & temperatura absoluta \\
\hline$N_{V}$ & densidade de estados efetiva da banda de valência \\
\hline$m_{l}^{*}$ & massa efetiva das lacunas \\
\hline$E_{g}$ & energia de banda proibida \\
\hline$H$ & Hamiltoniano \\
\hline$V_{g s}$ & tensão porta-fonte \\
\hline$F_{j}(x)$ & integral de Fermi-Dirac completa \\
\hline$L$ & comprimento da super-rede \\
\hline$V_{0}$ & altura da barreira \\
\hline$m_{0}$ & massa do elétron livre \\
\hline $\mathbf{k}$ & vetor de onda \\
\hline$E_{l}$ & energia longitudinal \\
\hline$E_{t}$ & energia transversal \\
\hline$I$ & amplitude da onda incidente \\
\hline$R$ & amplitude da onda refletida \\
\hline$\tau$ & amplitude da onda transmitida \\
\hline$A$ & constante de normalização \\
\hline$\rho(\mathbf{k})$ & densidade de ondas incidentes \\
\hline$A i$ & funções de Airy de primeiro tipo \\
\hline$B i$ & funções de Airy de segundo tipo \\
\hline $\mathbf{W}$ & Wronskiano \\
\hline $\mathbf{W}^{*}$ & conjugado do Wronskiano \\
\hline$m$ & número quântico magnético \\
\hline$n_{r}$ & número quântico radial \\
\hline
\end{tabular}


xvi

$J_{m} \quad$ função de Bessel

$\chi_{n_{r} m}$ raíz da função de Bessel

$F \quad$ campo elétrico

$E_{p h}$ energia do fóton

$E_{G}$ energia de banda proibida do material 


\section{Capítulo 1}

\section{Introdução}

A invenção do transistor em 1947 causou um impacto enorme no nosso cotidiano e no desenvolvimento econômico mundial, a ponto de ser considerada uma das mais importantes invenções do século XX. Em pouco mais de 20 anos, o transistor levou à extinção de toda uma era tecnológica baseada nas válvulas, que até então compunham todos os aparelhos eletroeletrônicos disponíveis, tais como rádios, televisões, radares, dentre outros. Sem dúvida alguma, a fabricação de transistores baseados em materiais semicondutores possibilitou o surgimento de aparelhos com menores dimensões, peso e consumo de energia, mais rápidos e eficientes, diferentemente das válvulas, abrindo uma nova era tecnológica e comercial, culminando no surgimento do computador de mesa - desktop.

Já na década de 60 a eletrônica possuia um vasto repertório de dispositivos e fenômenos físicos conhecidos, com muitos aspectos teóricos já bem estabelecidos para produzir muitos produtos interessantes. Por exemplo, aproveitando o caráter dual e a natureza quântica dos dispositivos eletrônicos tem-se, em 1958, o surgimento do primeiro dispositivo concebido e construído levando em conta estas características: o diodo túnel de Leo Esaki [1], que observou o efeito de resistência negativa em junções p-n altamente dopadas, originando, assim, uma nova classe de dispositivos que exibiam o fenômeno quântico de tunelamento. Este dispositivo proporciona uma alta velocidade acompanhada de um baixo consumo de potência, oferecendo assim uma promessa futura para o desenvolvimento de dispositivos ultra-rápidos. Do ponto de vista tecnológico, efeitos quânticos podem ser convenientemente usados para se construir dispositivos eletrônicos e/ou optoeletrônicos. Especificamente, há três manifestações importantes de efeitos quânticos que podem ser empregadas para se projetar dispositivos, são elas : 
- Quantização dos níveis de energia,

- Tunelamento quântico e

- Interferência quântica.

$\mathrm{Na}$ realidade, pode-se usar uma ou mais das propriedades acima para a produção de dispositivos com características úteis. Mais recentemente, foi criado o conceito de dispositivos quânticos, que se refere exatamente a todos os dispositivos cuja a base de funcionamento fundamenta-se em algum dos efeitos quânticos citados acima. Em geral, dispositivos que utilizam efeitos de quantização para seu funcionamento são dispositivos de alto impacto tecnológico, e objeto de grande interesse de investigação científica atual, pois oferecem possibilidade de serem absorvidos na eletrônica convencional [2]-[4].

No entanto, na prática, a implementação de muitas dessas possibilidades esbarrou inicialmente num problema muito significativo. A tecnologia do início da era eletrônica não estava desenvolvida o suficiente para aproveitar todo o conhecimento teórico até então conquistado. De fato, este cenário somente começou a ser modificado a partir de meados da década de 70 , quando muitas implementações se tornaram possíveis, devido ao desenvolvimento das técnicas de crescimento de materiais, dos quais destaca-se o MBE (Molecular Beam Epitaxy) e o MOCVD (Metalorganic Chemical Vapor Deposition), que visam a fabricação de estruturas de heterojunções de semicondutores dos grupos (III)-(V), entre outros. O domínio destas técnicas possibilitou o crescimento epitaxial de finas camadas de semicondutores compostos com a capacidade de controle de espessura das mesmas em escala atômica, inserindo os dopantes apenas nas regiões desejadas, sendo possível construir diferentes heteroestruturas semicondutoras, tais como sistemas AlGaAs/GaAs, InAlAs/InGaAs, Si/Ge, com alto grau de pureza, permitindo a implementação de dispositivos para várias funções. Foi possível, assim, construir uma grande variedade de microestruturas com dimensões comparáveis ao comprimento de onda de de Broglie dos elétrons, revelando, desta forma, a natureza quântica presente nestes sistemas e possibilitando a investigação da física em sistemas com dimensões reduzidas, da ordem de dezenas de Angstrons, nas quais os efeitos quânticos tornam-se relevantes.

Prontamente, heteroestruturas de dopagem modulada tais como a mostrada na Figura (1.1) e propostas teoricamente por Esaki e Tsu [5], em 1969, conhecidas como superredes, tornaram-se experimentalmente possíveis. O interesse por tais heteroestruturas reside no fato dessa construção separar os elétrons de condução das impurezas ionizadas, consequentemente 
reduzindo o espalhamento Coulombiano, e aumentando a mobilidade dos portadores de carga, confinados em poços quânticos na escala de aproximadamente $10 \mathrm{~nm}$. Nestas condições, essas estruturas formam estados eletrônicos acoplados, induzindo inúmeros efeitos quânticos, tais como confinamento quântico dos elétrons, efeito Hall quântico, efeito Stark quântico-confinado, oscilações de Bloch, e emissão e detecção de fótons com base em transições inter sub-banda de elétrons. Um outro fenômeno significativo que pode ser verificado, provindo da física resultante das múltiplas camadas periódicas de heteroestruturas semicondutoras com camadas ultrafinas, é o fenômeno da resistência negativa [5]. Nesta classe de dispositivos estão os dispositivos de tunelamento ressonante, notadamente, os RTDs ("Resonant Tunneling Diode") [6], os quais são caracterizados pela não linearidade na característica corrente-tensão e pela diminuição do tempo de resposta, utilizados em circuitos oscilantes nos quais o efeito da resistência negativa compensa a perda ôhmica, com aplicações em circuitos lógicos e dispositivos de memória. Sendo assim, estruturas de tunelamento apresentam atualmente um elevado grau de maturidade tecnológica, em que um grande número delas já operam a temperatura ambiente [7].

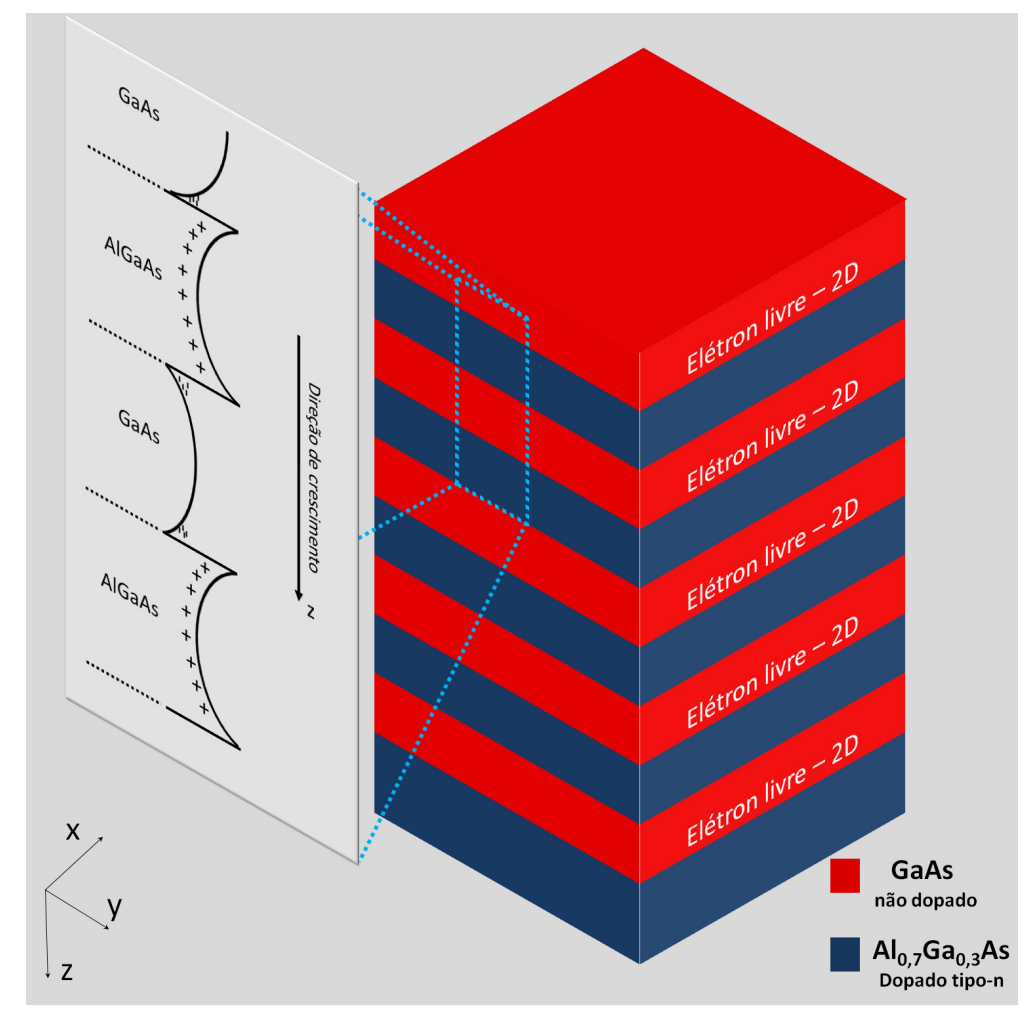

Figura 1.1: Diagrama esquemático de uma super-rede de dopagem modulada mostrando as camadas em que os elétrons ficam confinados, o gás de elétrons bidimensional (2D). 
De outro lado, a possibilidade de se confinar elétrons espacialmente em camadas extremamente finas de forma controlada usando heteroestruturas semicondutoras permitiu também a construção e desenvolvimento dos transistores de efeito de campo ("field-effect-transistorFET), os quais apresentaram um ganho significativo nas propriedades de transporte dos sistemas 2-DEG ("2 - Dimensional Electron Gas") quando se empregou o esquema de dopagem modulada, em que a camada de inversão é quantizada na direção de crescimento das camadas epitaxiais. Desta forma, já no início de 1990, os "High Electron-Mobility Transistors- HEMTs, outra denominação para FETs baseados nesse princípio, demonstraram amplificação de potência bem acima de $100 \mathrm{GHz}$ com excelentes características de ruído.

Como alternativa, restringindo-se mais uma vez os graus de liberdade do elétron no gás de elétrons bidimensional, 2-DEG, em outra direção, valendo-se de toda a tecnologia atual de fabricação de estruturas de dimensionalidade reduzida, pode-se fabricar um gás de elétrons unidimensional (1-DEG). Estas estruturas são geralmente chamadas de fios quânticos. Do ponto de vista teórico, o conceito de fio quântico foi introduzido por Sakaki em 1980 [8], mas não foi senão na década de 90 que essas estruturas se tornaram tecnologicamente possíveis, com o desenvolvimento das técnicas de crescimento de materiais e de litografia sub-mícron para fabricação de dispositivos. Nestas estruturas o confinamento do elétron é bidimensional, enquanto o movimento na terceira direção é livre, ocorrendo supressão do espalhamento elástico por impurezas nos fios, fato mostrado teoricamente por Sakaki em 1980 [8], resultando na alta mobilidade do elétron num sistema unidimensional (1D), e contribuindo desta maneira para a produção de dispositivos ultra-rápidos [9], [10] e [11].

O objetivo primordial desta dissertação é estudar dispositivos de fio quântico empregando o mecanismo de tunelamento ressonante. Para isso será realizado um levantamento bibliográfico para compreender o estado da arte dos dispositivos encontrados na literatura.

Neste ponto, devido ao altíssimo desenvolvimento tecnológico atuais, não podemos deixar de mencionar que, de fato, a micro e a nanoeletrônica atual apresentam dois tipos de abordagens para produção de dispositivos de fios quânticos, conhecidas como "top-down"e "bottomup", as quais foram primeiramente sugeridas pelo Foresight Institute em 1989, com o objetivo de entender as diferenças entre o processo de fabricação molecular e o processo tradicional [12].

No processo de fabricação convencional, o qual designamos por "top-down", o dispositivo é produzido a partir de um material volumétrico ("bulk"), e alterado convenientemente até produzir a nanoestrutura desejada, caracterizando assim uma abordagem determinística [12], 
com grande complexidade e exatidão no processo de fabricação. Há uma variedade de processos que são utilizados para se produzir o material inicial que dará origem ao dispositivo desejado, tais como litografia, corrosão, etc.

Por outro lado, processos de fabricação denominados "bottom-up", representam uma abordagem estocástica [12], ou seja, as nanoestruturas, crescidas átomo por átomo, não possuem uma ordenação uniforme, obrigando assim um desenvolvimento de alto nível dos instrumentos de visualização e manipulação, os quais permitirão a construção dos componentes dos dispositivos desejados.

\subsection{Abordagem top-down}

No âmbito da abordagem "top-down"realizou-se um levantamento bibliográfico acerca da evolução dos dispositivos semicondutores, dentro do escopo deste trabalho, desde meados dos anos 90 até os dispositivos atuais. Primeiramente vamos discutir o trabalho de Snider et al [13], de 1990, um trabalho essencialmente teórico, no qual propõem um transistor de fio quântico construído a partir do confinamento produzido por heteroestruturas semicondutoras (2-DEG), seguido por um segundo confinamento (1-DEG) produzido por contatos metálicos distantes entre si de uma distância $d$, compondo assim uma estrutura de eletrodos chamada "split-gate", depositada sobre uma heteroestrutura convencional de AlGaAs/GaAs e dando origem a contatos Schottky, como mostrado na Fig.(1.2), à esquerda. Por meio do contato Schottky, o dispositivo pode ser polarizado com uma tensão $V_{c}$ com respeito ao substrato, de modo a remover o canal 2-DEG por baixo do contato metálico, como indicado na Fig.(1.2), à direita. Vale observar que, nesta situação, a largura do fio pode variar continuamente através da variação da tensão aplicada no contato, $V_{c}$, por remoção dos portadores de carga entre os dois contatos metálicos. As características eletrônicas da estrutura, tais como perfil de potencial $V(x, y)$, concentração de portadores $n(x, y)$, e níveis de energia $\varepsilon_{i}$, são obtidos a partir do desenvolvimento do cálculo auto-consistente das equações de Schrödinger e Poisson para fios quânticos, num formalismo que considera a quantização dos níveis de energia em duas dimensões.

Outra proposta encontrada na literatura [14], para a fabricação de heteroestruturas de fios quânticos a partir de heterojunções semicondutoras, é baseada em variações das técnicas de litografia e de "etching", as quais produzem corrosões no material semicondutor, como mostrado na Fig.(1.3), resultando em um confinamento eletrostático, onde a largura do fio quântico pro- 

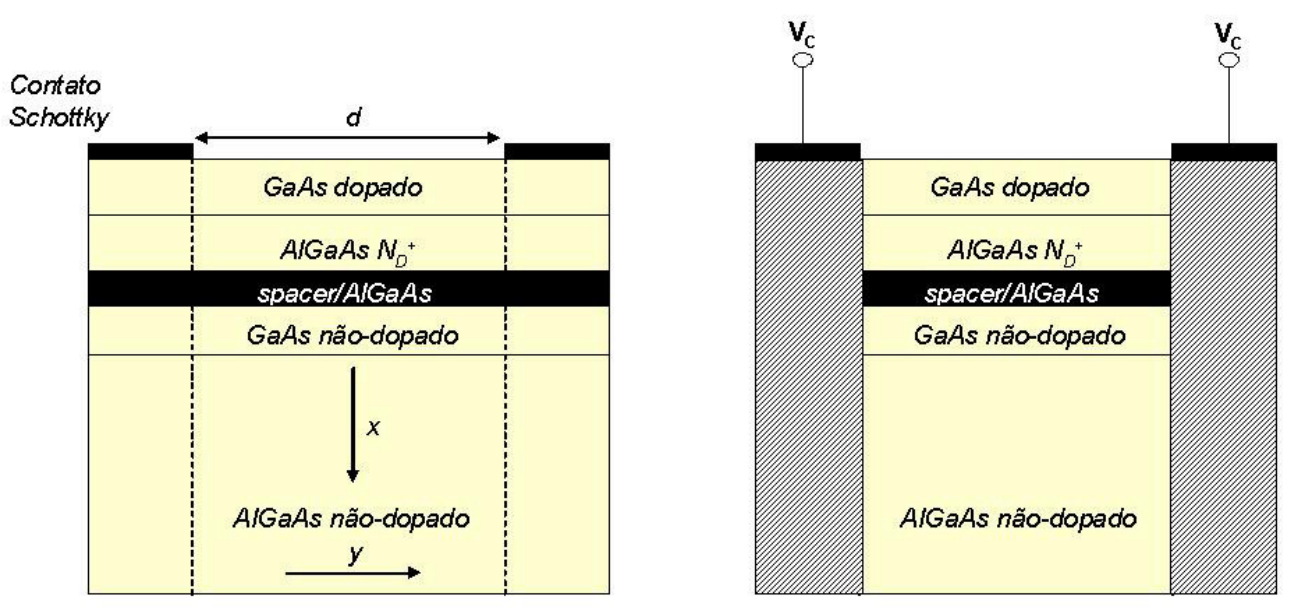

Figura 1.2: Geometria do dispositivo apresentando uma estrutura de "split-gate" sobre uma heteroestrutura convencional de AlGaAs/GaAs, em que os eletrodos de contato são produzidos, e pelos quais pode-se polarizar o dispositivo com relação ao substrato. Pode-se imaginar uma situação, como ilustrada à direita, em que a tensão aplicada aos contatos, $V_{c}$ remova por completo o canal 2-DEG, abaixo do contato metálico, podendo em seguida variar a largura do fio além da distância $d$.

duzido depende essencialmente dos parâmetros $d_{p}$ e $w_{d}$, mostrados na Fig.(1.3), estabelecidos previamente na fabricação.

Como pode ser observado, a partir do domínio das técnicas de crescimento e de nanolitografia podem-se construir várias heteroestruturas com diversos sistemas de materiais e dopagens, para fabricação de inúmeros dispositivos quânticos, de dimensões nanométricas.

Ainda nesta linha, Islam et al. [9] em 1996, propõem um HEMT de fio quântico, como mostrado esquematicamente na Fig.(1.4). A heteroestrutura, ao longo da direção x, consiste de uma camada de $\mathrm{Al}_{0,7} \mathrm{Ga}_{0,3}$ As não-dopada, de espessura da ordem de $300 \AA$, depositada sobre um substrato de GaAs, seguida por uma camada de 140 A de GaAs, onde será produzido o poço quântico, uma camada espaçadora de $40 \AA$ de $\mathrm{Al}_{0,7} \mathrm{Ga}_{0,3} \mathrm{As}$ não-dopada, e uma camada de $\mathrm{Al}_{0,7} \mathrm{Ga}_{0,3}$ As dopada tipo $\mathrm{n}$, de cerca de uns $1000 \AA$. Na direção z, existem dois contatos laterais, que podem ser obtidos através de técnicas de corrosão e de implantação de íons, os quais proporcionam o confinamento unidimensional. Desta forma, os elétrons ficam então confinados nas direções x e z, movendo-se livremente na direção y, resultando no gás de elétrons unidimensional (1-DEG), levando a uma altíssima mobilidade de portadores eletrônicos.

Islam et al. [9] apresentaram um modelo analítico auto-consistente para a predição 


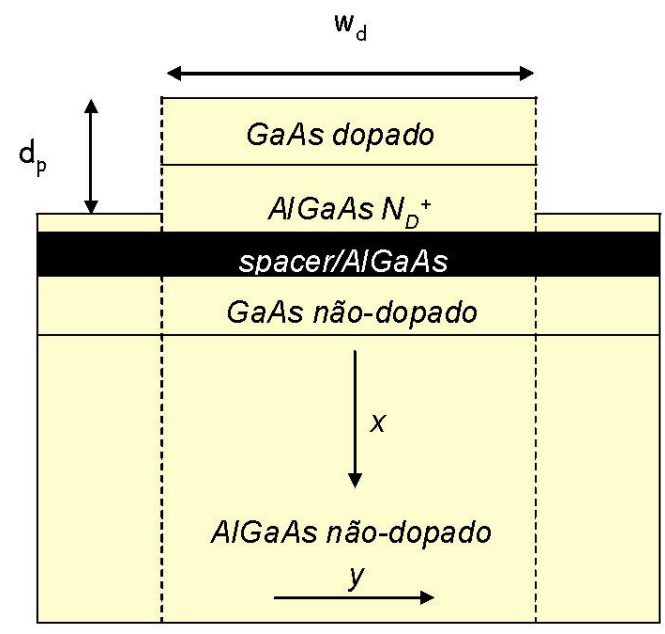

Figura 1.3: Nesta estrutura a corrosão provocada sobre a superfície da heteroestrutura é capaz de provocar o confinamento eletrônico, dando origem a um fio quântico.

das caracterísitcas elétricas de HEMTs de fio quântico, incluindo a densidade de portadores no canal 1DEG, como também as funções de ondas eletrônica, dentro e fora do fio quântico e os níveis de energias. Na direção x, o confinamento produzido na heterointerface AlGaAs/GaAs foi modelado por um poço de potencial triangular, enquanto que, na direção y, o confinamento foi modelado no âmbito da aproximação retangular, produzida pelo confinamento eletrostático gerado pelo potencial longitudinal de inserção dos contatos, mostrados na Fig.(1.4).

Como usual, a concentração do gás de elétrons unidimensional é controlada pela aplicação da tensão de porta, gerando assim, a relação de controle de carga. Pela implantação dos contatos de fonte e dreno, pode ser obtido o efeito transistor na estrutura de gás de elétron unidimensional, assim resultando em um HEMT fio quântico, como mostrado na Fig.(1.4). Nesse trabalho, os autores ainda obtém a característica corrente-tensão, I-V, e a transcondutância, as quais são obtidas estendendo-se os modelos de HEMTs convencionais para os HEMTs de fios quânticos. Em particular, encontra-se que a transcondutância em transistores de fio quântico é, pelo menos, duas vezes maior do que nos dispositivos 2D.

Em linhas gerais, a família de transistores de fios quânticos como os propostos por Islam et al. [9] encontram inúmeras aplicações e são ainda estudados [15],[16], para vários outros sistemas de materiais, levando-se também em conta vários outros efeitos que, dependendo da estrutura, podem tornar-se significativos.

Tratando essencialmente da melhoria da performance dos dispositivos semicondutores, 


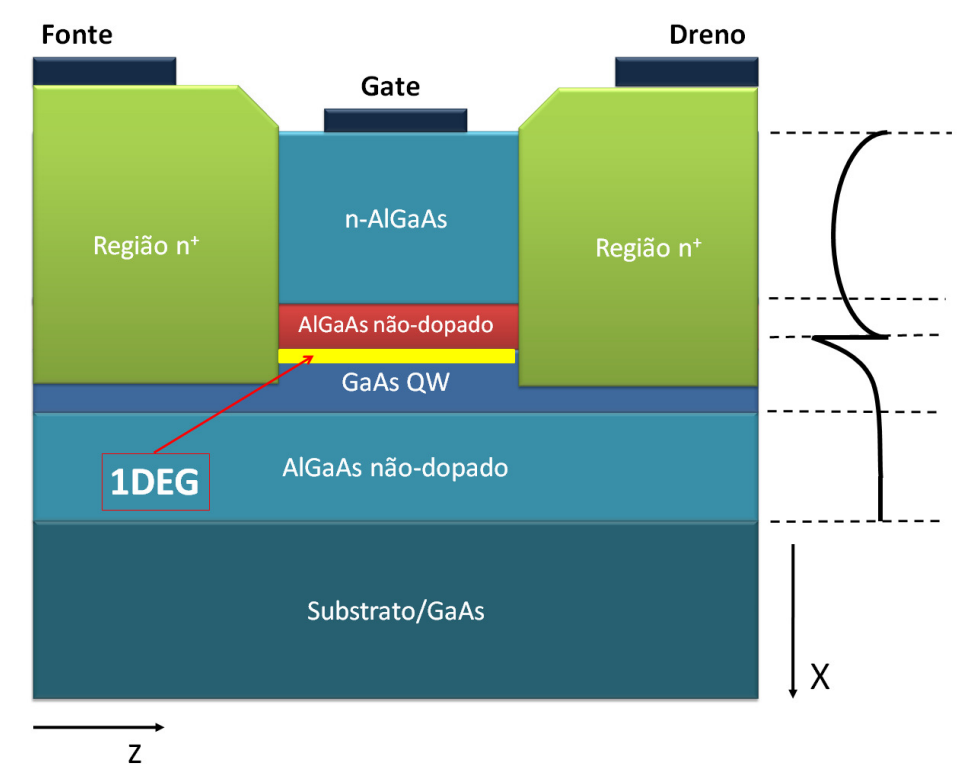

Figura 1.4: Seção transversal de um HEMT de fio quântico, proposto por Islam et al na Ref. $[9]$.

vê-se na literatura inúmeras propostas [17] de unir as vantagens dos sistemas que empregam fios quânticos, notadamente a comprovada supressão do espalhamento de fônons [8], com as propriedades únicas das estruturas de tunelamento ressonante. Trata-se de uma interessante combinação que vem de encontro ao crescente interesse por dispositivos de operação ultra-rápida, e que apresentam resistência diferencial negativa.

Neste sentido, Yang et al [18], em 1989, discutem as características de um transistor de tunelamento ressonante de efeito de campo de duplo poço quântico, como mostrado na Fig.(1.5). O dispositivo é obtido por fotolitografia padrão e técnicas de corrosão, e assemelha-se ao dispositivo proposto por Islam et al. [9], senão pela sequência de camadas semicondutoras que agora incorpora uma estrutura de tunelamento ressonante e a ausência dos contatos laterais que produzem o confinamento unidimensional. A heteroestrutura de duplo poço quântico é crescida sobre um substrato de $\mathrm{n}^{+}$-GaAs por epitaxia de feixe molecular. Em seqüência, uma camada "buffer" de GaAs não dopada com espessura de $2 \mu m$, é crescida sobre o substrato, seguida por uma barreira de $\mathrm{Al}_{0,37} \mathrm{Ga}_{0,63} \mathrm{As}$ não dopado de espessura de $8000 \AA$, um poço quântico de $\mathrm{n}^{+}$GaAs $\left(1,0 \times 10^{18} / \mathrm{cm}^{3}\right)$ de espessura de $200 \AA$, uma barreira não dopada de $\mathrm{Al}_{0,37} \mathrm{Ga}_{0,63} \mathrm{As}$ de $30 \AA$, outro poço quântico de GaAs não dopado de $70 \AA$, mais uma barreira não dopada de 
$\mathrm{Al}_{0,37} \mathrm{Ga}_{0,63} \mathrm{As}$ de $30 \AA$, uma camada de $\mathrm{n}^{+}$-GaAs $\left(1,0 \times 10^{18} / \mathrm{cm}^{3}\right)$ com espessura de $1500 \AA$ e, por fim, uma camada no topo da heteroestrutura, de $500 \AA$ de $n^{+}-\operatorname{In}_{0,45} \mathrm{Ga}_{0,55} \mathrm{As}$, denominada "cap layer". Para finalizar o dispositivo, é utilizado a fotolitografia padrão para definir a região do dreno, e a deposição de $\mathrm{Ti} / \mathrm{Pt} / \mathrm{Au}$ para formar os demais contatos: dreno, fonte, gate do topo e gate de baixo.

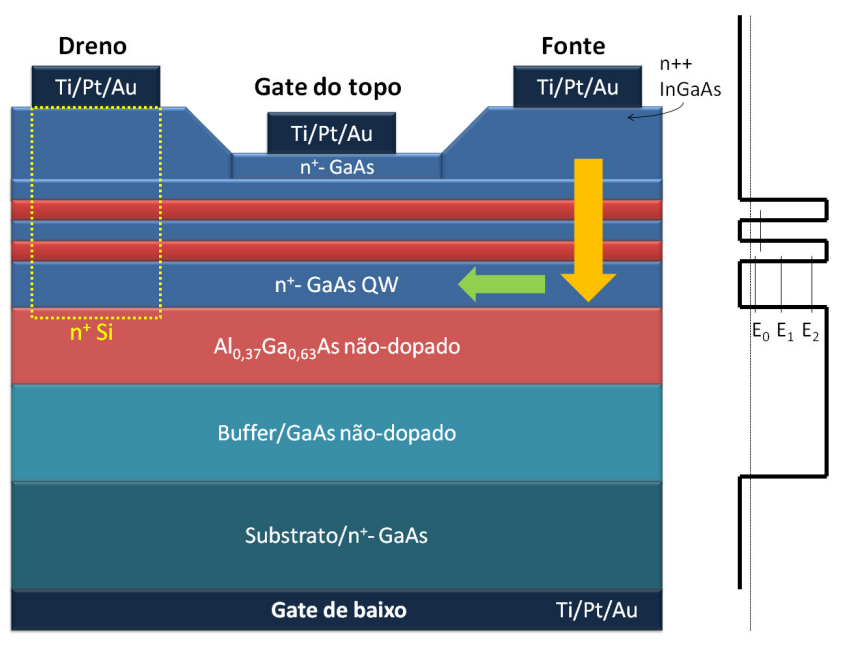

Figura 1.5: Esquema da seção transversal de um transistor de tunelamento ressonante de efeito de campo de duplo poço quântico (fora de escala). A área compreendida pela linha seccionada amarela representa o implante de $\mathrm{n}^{+}-\mathrm{Si}$. O tunelamento é representado pela seta dourada, enquanto que a flecha verde representa o caminho da corrente no poço quântico (QW) de GaAs. Dispositivo proposto por Yang et al na Ref. [18].

A estrutura proposta por Yang et al [18] é similar ao tunneling transfer field-effect transistor [19], trabalho pioneiro na área de dispositivos que apresentam transcondutância negativa, efeito que surge em dispositivos onde a corrente oscila, apresentando inúmeros picos. Yang et al [18] demonstraram que o transistor proposto apresenta propriedades muito interessantes, revelando particularidades na transcondutância, tais como presença de picos, oscilações e transcondutância negativa.

Conforme já mencionado, a grande vantagem de se construir transistores baseados em tunelamento quântico é que podem operar em velocidades maiores do que os convencionais. Se no esquema da Figura (1.5) for realizado um confinamento eletrostático através de contatos metálicos, como mostrado no esquema proposto por Islam et al [9], sem dúvida, poderá ser 
verificado uma melhoria ainda maior no desempenho do dispositivo, uma vez que, as estruturas formadas por fios quânticos minimizam o espalhamento elástico.

Outros dispositivos incorporando estruturas ressonantes e exibindo o fenômeno de diferencial de transcondutância negativa foram reportados [20]-[23]. De fato, o interesse por estruturas de tunelamento ressonante vai além de estruturas convencionais, tais como FETs e transistores bipolares, havendo diversos trabalhos explorando as características das estruturas de tunelamento para produzir dispositivos com características únicas [24]-[26].

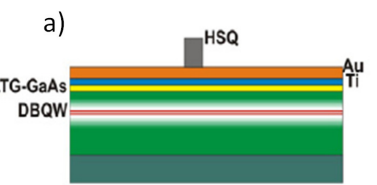

b)

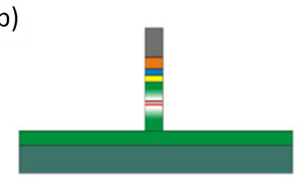

c)

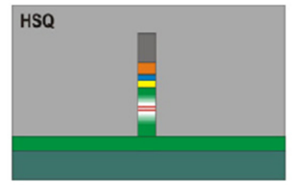

d)

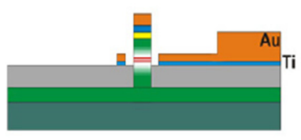

e)

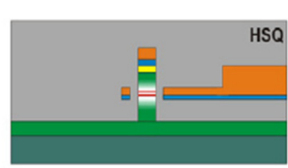

f)

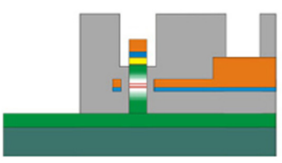

g)

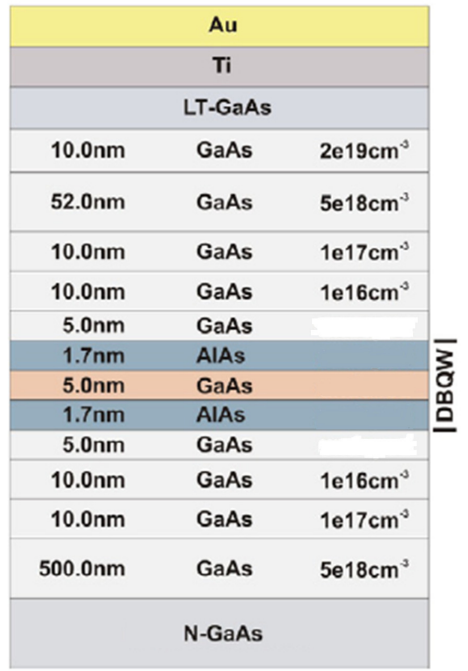

Figura 1.6: Representação esquemática dos passos de fabricação dos transistores de tunelamento ressonante de nanocoluna. a) crescimento das camadas via MBE, metalização e litografia de feixe eletrônico. b) Definição da nanocoluna por "etching". c) Planarização com várias camadas de HSQ (hydrogen silsesquioxan). d) Etching de plasma isolando a camada de HSQ e litografia de feixe eletrônico para formar o gate. e) Segunda planarização com várias camadas de HSQ. f) Exposição dos contatos do topo da nanocoluna e o gate. g) Esquema das heteroestruturas com uma dupla barreira (AlAs), um poço quântico (GaAs) e regiões de contatos. Esquemas retirados do trabalho de Wensorra, Ref. [27].

O dispositivo proposto por Wensorra et al, Ref. [27] em 2009, é um transistor de tunelamento ressonante de nanocoluna, mostrado nas Figs.(1.6) e (1.7). Entende-se por nanocoluna, nanoestruturas de confinamento quântico unidimensional, tais como os fios quânticos.

Na Ref. [27], Wensorra et al., como pode ser verificado na Fig.(1.6), desenvolvem o transistor de tunelamento ressonante de nanocoluna, onde uma dupla barreira é introduzida entre as camadas espaçadoras de GaAs e camadas tipo-n de GaAs com um aumento gradual 

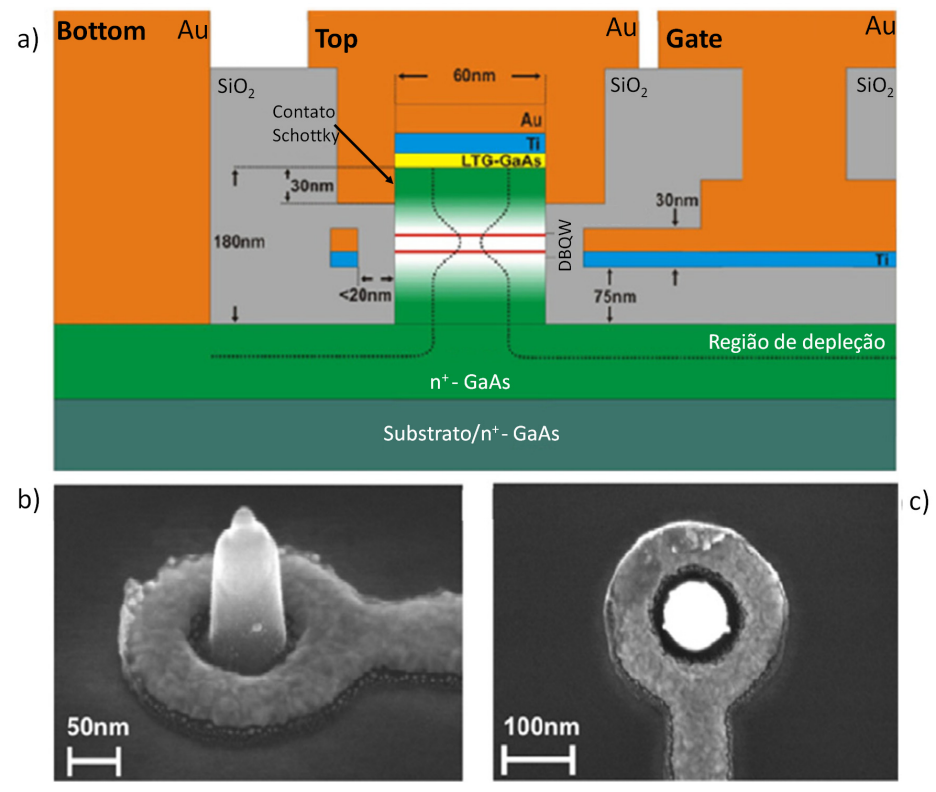

Figura 1.7: a) Visão esquemática do transistor de tunelamento ressonante de nanocoluna. Micrografias SEM do RTT de nanocoluna: b) vista de fora e c) vista de cima. Micrografias e esquemas retirados do trabalho de Wensorra, Ref. [27].

das dopagens até o topo e a base respectivamente. O crescimento epitaxial termina com uma camada altamente dopada $\left(n^{++}=2 \times 10^{19} \mathrm{~cm}^{-3}\right)$ de $10 \mathrm{~nm}$ de espessura, uma camada de GaAs crescida a baixa temperatura (LTG) e por fim a metalização com Ti/Au que é depositada ao término do crescimento das heteroestruturas, formando assim um excelente contato ôhmico para este nanodispositivo. Posteriormente, é efetuado um "etching", com o intuito de formar a nanocoluna com diâmetros entre $30 \mathrm{~nm}$ e $1 \mu \mathrm{m}$, definidas pela litografia de feixe de elétrons usando o hydrogen silsesquioxan (HSQ), "high-resolution inorganic negative electron-beam resist", mostrado na Fig.(1.6-a). HSQ é um material que depois de exposto aos elétrons, tem uma estrutura amorfa similar ao $\mathrm{SiO}_{2}$. A definição da nanocoluna é o próximo passo, mostrado na Fig.(1.6-b) onde uma fina camada de Au é depositada via "sputtering"com íons de $\mathrm{Ar}+$. Na seqüência, HSQ é usado para planarização e isolação física da nanocoluna, Fig.(1.6-c), subsequentemente é construído um gate metálico em torno da nanocoluna, consistindo de camadas de $5 \mathrm{~nm}$ de Ti e $15 \mathrm{~nm}$ de Au, como mostrado na Fig.(1.6-d). Depois da fabricação do gate, uma segunda planarização com várias camadas de HSQ é realizada, Fig.(1.6-e). Litografia óptica é utilizada para abrir janelas para expor os contatos e o gate, Fig.(1.6-f). Por fim os contatos são processados. Já na Fig.(1.7) tem-se o esquema da seção transversal do dispositivos após o processo de fabricação. Com a obtenção do transistor de tunelamento ressonante de nanocoluna, 
tem-se a noção da evolução dos dispositivos semicondutores que estão agregados no âmbito da abordagem "top-down".

\section{$1.2 \quad$ Abordagem bottom-up}

Do ponto de vista da outra abordagem denominada "bottom-up", os fios quânticos semicondutores foram primeiramente explorados por Hiruma et al. em 1991 [28], que realizou o crescimento controlado e a caracterização elétrica e óptica de um grande conjunto de fios quânticos semicondutores. Desde então houve um interesse ainda maior por sistemas unidimensionais, devido ao crescente número de aplicações em dispositivos de alta velocidade de processamento aliado com o baixo consumo de potência [29].
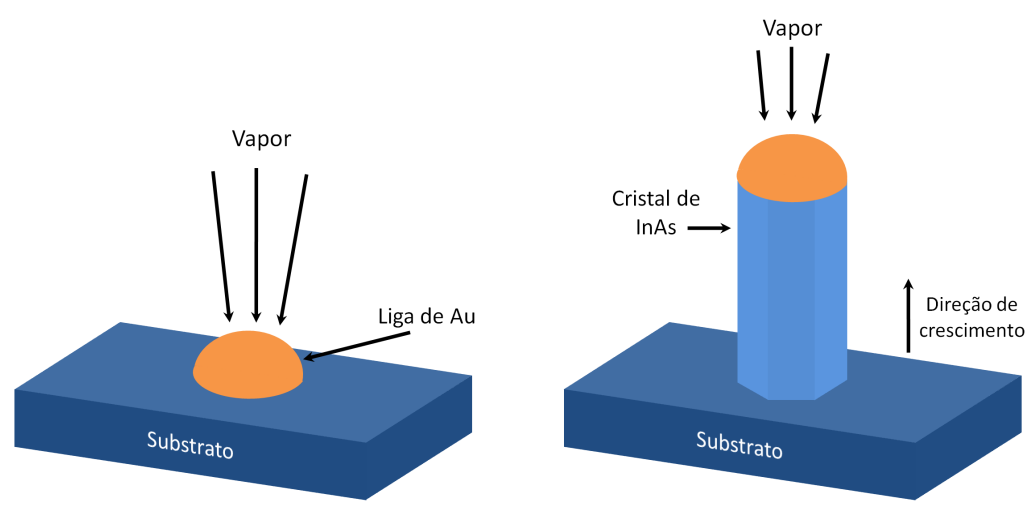

Figura 1.8: Ilustração esquemática do mecanismo VLS (vapor-liquide-solid) de crescimento de nanofios de InAs. O crescimento do cristal é ilustrado basicamente em dois passos: o primeiro passo envolve o sistema vapor-liquído, no qual a transferência de material da fase de vapor ocorre diretamente na gota de Au em fase líquida, com a finalidade de supersaturar a gota líquida com o material do cristal de InAs. Já o segundo passo ocorre no sistema líquido-sólido através da precipitação do material contido na liga de Au, que atua como um catalisador e um guia para o crescimento do material na interface líquido-sólido. Esquema retirado do livro de M. Meyyappan e M. K. Sunkara, Ref. [32].

Sendo assim, Ohlsson et al. em 2001, Ref. [30], descreve o processo de fabricação de um fio quântico semicondutor crescido sobre um substrato de GaAs usando epitaxia de feixe químico à partir de partículas de ouro com tamanho selecionado. Seguindo o processo de fabricação de Ohlsson, Thelander et al., Ref. [31], em 2004, constrói os fios quânticos homôgeneos de InAs, de 
acordo com o processo descrito na Fig.(1.8), detalhado no M. Meyyappan e M. K. Sunkara, Ref. [32], e posteriormente introduz barreiras de potencial de InP, no qual o processo de construção de fios quânticos com heteroestruturas é esquematizado na Fig.(1.9) que ilustra o trabalho desenvolvido por Gudiksen et al, Ref. [33]. É viabilizada então a construção de dispositivos de fios quânticos com heteroestruturas semicondutoras de tunelamento ressonante, como fios quânticos homogêneos de InAs, fios quânticos de InAs com uma barreira de InP, dispositivos de fios quânticos de InAs com dupla barreira de InP e transistores de um único elétron (SET). Alguns destes dispositivos podem ser analisados na Fig.(1.10).

a)

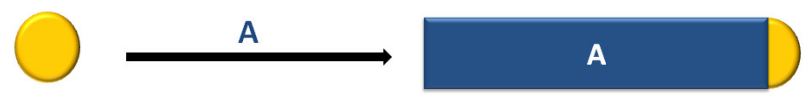

b)

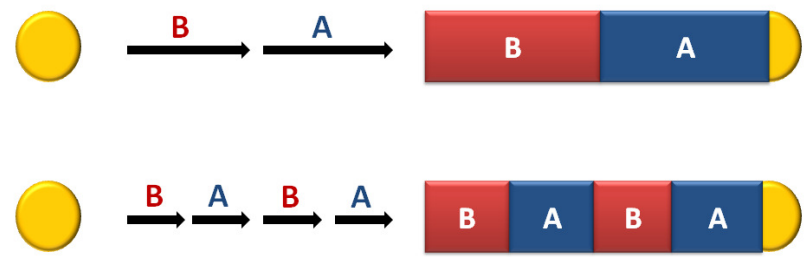

Figura 1.9: Síntese de um fio quântico de heteroestruturas, onde o círculo dourado representa a liga de ouro, conhecida como catalisador, a) crescimento de um fio quântico semicondutor homogêneo de InAs; b) crescimento de um segundo fio quântico semicondutor de InP; c) repetição dos passos anteriores para a criação de um fio quântico de heteroestruturas semicondutoras.

Portanto, estágio atual de maturidade tecnológica permite produzir tais dispositivos de fios quânticos com tunelamento ressonante tanto usando a abordagem "top-down"[35]-[41] como a "bottom-up"[42]-[46]. Micrografias de nanoestruturas construídas pelas diferentes abordagens estão mostradas na Fig.(1.11).

Sendo assim, esta dissertação, voltada para a modelagem de transistores de fio quântico com tunelamento ressonante, foca em dois métodos muito utilizados na literatura para descrever dispositivos semicondutores, a saber, um método numérico, obtido a partir da solução autoconsistente das equações de Schrödinger e Poisson [13], [47], e um método analítico baseado na Matriz Transferência [48], [49]. Especificamente, para compreender melhor os mecanismos de transporte e as propriedades elétricas dos dispositivos quânticos de interesse, passamos a descrever, a seguir, como está organizado este trabalho. 
a)

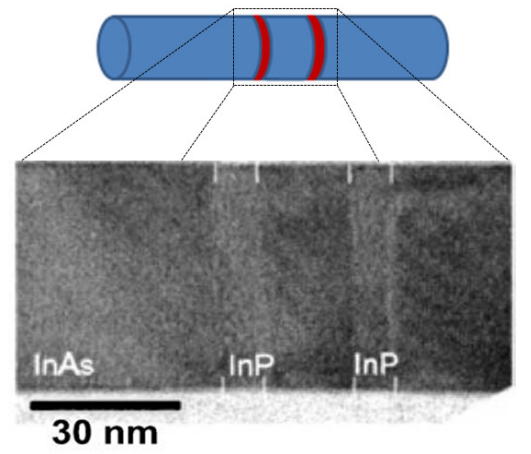

b)

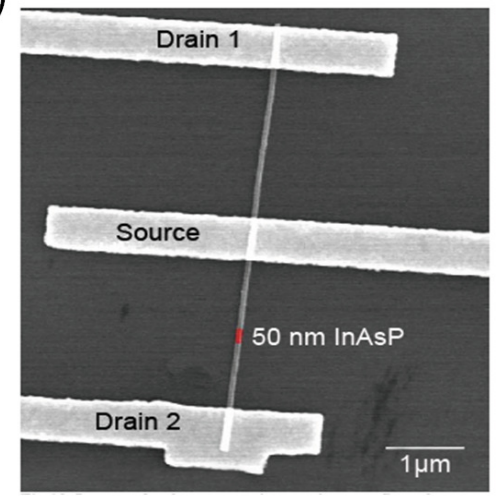

Figura 1.10: Exemplos de dispositivos construídos por técnicas de fabricação dentro da abordagem "bottom-up": a) Microscopia eletrônica mostrando um fio quântico de InAs com duas barreiras de InP, igualmente espaçadas, retirado do trabalho de Thelander et al, na Ref. [31].b) Esquema de um transistor de fio quântico planar com heteroestrutura, onde uma barreira de 50 nm de InAsP foi introduzida no fio quântico de InAs, retirado do trabalho de Wernesson et al, na Ref. [34].

No Capítulo 2 é desenvolvido um formalismo numérico baseado na solução autoconsistente das equações de Schrödinger-Poisson para resolver sistemas envolvendo poços e fios quânticos. A ferramenta computacional que implementa tal formalismo foi desenvolvida pelo nosso grupo de pesquisa [50]-[52], e, neste capítulo, é utilizada para se encontrar os auto-estados, os níveis de energia, e as densidades eletrônicas relacionadas a transistores de fios quânticos produzidos usando-se técnicas "top-down", a partir de técnicas de litografia e corrossão em estruturas do tipo "split-gate".

No Capítulo 3 realizou-se um estudo analítico de transporte de corrente em heteroestruturas semicondutoras tipo super-redes, contendo uma seqüência arbitrária de barreiras e poços quânticos, com e sem a aplicação de campo elétrico externo, tendo como base os trabalhos de Tsu e Esaki [53]. Estes trabalhos pioneiros mostraram que estas heteroestruturas exibiam o fenômeno de resistência negativa [5], calculando a expressão para a densidade de corrente, J, fluindo através da estrutura quando uma tensão é aplicada.

Tais cálculos exigem o conhecimento prévio do coeficiente de transmissão, ou da probabilidade de tunelamento, T, dos elétrons através das heteroestruturas, para uma correta compreensão do fenômeno de transporte, que, nesta situação em especial, tem no tunelamento um papel fundamental. O cálculo do coeficiente de transmissão dos elétrons para tais sistemas é re- 

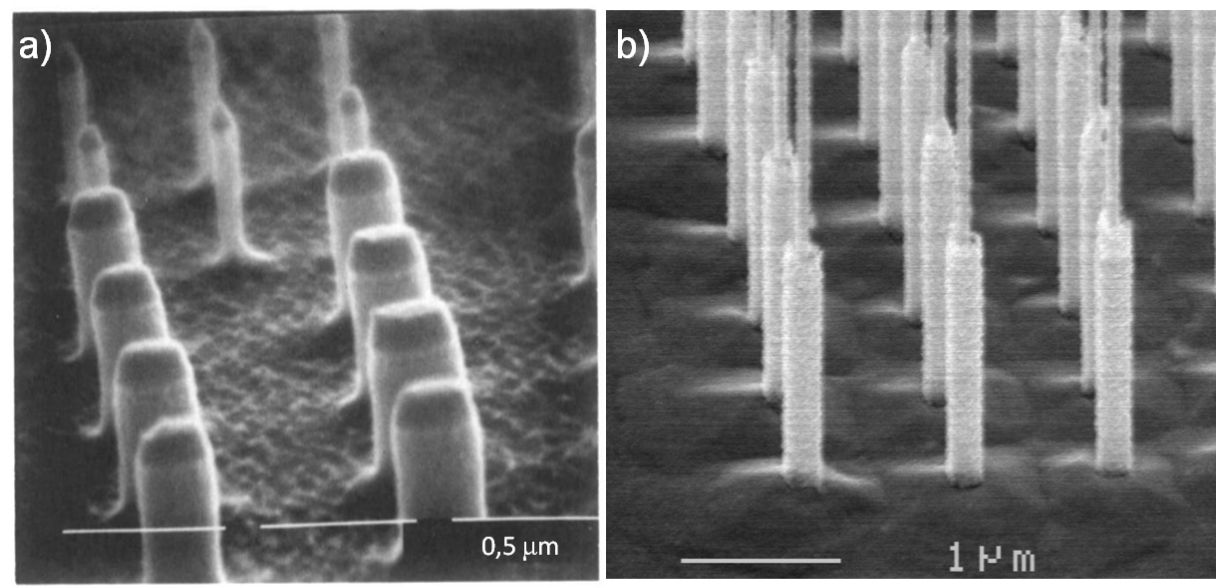

Figura 1.11: a) Micrografia eletrônica de nanoestruturas de GaAs, [35], fabricados por técnicas pertencentes a abordagem "top-down". b) Micrografia eletrônica de fios quânticos de InAs, [34], fabricado por técnicas pertencentes a abordagem "bottom-up".

alizado usando-se o Método da Matriz Transferência (MMT), que, neste capítulo, é apresentado em detalhes.

A partir do conhecimento do coeficiente de transmissão é possível obter as curvas (I-V) para várias estruturas de interesse. Neste trabalho contrastamos nossos resultados com resultados encontrados na literatura [48], [53], [54], [55] e [56]. Por fim, o Método da Matriz Transferência é adequadamente adaptado para descrever também a heteroestruturas de simetria cilíndrica, realizando-se assim um estudo de transporte de corrente em um diodo de fio quântico de tunelamento ressonante.

No Capítulo 4, são apresentadas as conclusões deste trabalho. 


\section{Capítulo 2}

\section{Solução auto-consistente das}

\section{equações de Schrödinger-Poisson}

\section{para sistemas de fios quânticos}

Neste capitulo é apresentado um formalismo numérico para a solução de problemas envolvendo poços e fios quânticos, que é extremamente útil para se estudar as propriedades elétricas de transistores de efeito de campo baseados nestas estruturas. A análise numérica é auto-consistente, pois inter-relaciona o problema estático de se encontrar o potencial gerado através de uma distribuição de carga, com o problema de se obter a densidade de probabilidade das funções de onda dos elétrons que populam os estados quânticos associados ao potencial. Inicialmente é analisado apenas situações que envolvem poços quânticos, e finalmente, esse estudo é estendido para abranger também as estruturas que contém fios quânticos. Algumas características elétricas de transistores de fios quânticos são discutidas no final desse capítulo.

\subsection{Solução de sistemas envolvendo poços quânticos}

Uma solução mais exata para o cálculo das propriedades elétricas das super-heteroestru turas propostas no Capítulo 1, representada pela Fig.(1.4), pode ser obtida numericamente, através da solução auto-consistente das equações de Schrödinger e de Poisson, levando-se em conta uma série de efeitos que na aproximação analítica são geralmente desprezados. Nesta 
situação, pode-se escrever a equação de Schrödinger unidimensional de um elétron como

$$
\left[-\frac{\hbar^{2}}{2 a(x)} \frac{d}{d x}\left(\frac{a^{2}(x)}{m^{*}(x)} \frac{d}{d x} \frac{1}{a(x)}\right)+V_{e f}(x)\right] \Psi_{i}(x)=E_{i} \Psi_{i}(x)
$$

levando em conta, tanto a possível variação do parâmetro de rede $a(x)$ dos materiais envolvidos [57], como também a variação da massa efetiva $m^{*}(x)$ dos elétrons em cada material. Observe que, quando $a(x)$ e $m^{*}(x)$ são constantes com a posição, o primeiro termo da Eq.(2.1), o operador energia cinética recai no operador energia cinética usual $\left(-\hbar^{2} /\left(2 m^{*}\right)\right) d^{2} / d x^{2}$. O potencial efetivo $V_{e f}(x)$ representa o potencial percebido pelo elétron, e pode ser representado por

$$
V_{e f}(x)=\Delta E_{c}(x)-q V(x)+V_{x c}(x)+C_{1}\left(\epsilon_{x x}+\epsilon_{y y}+\epsilon_{z z}\right)
$$

em que $\Delta E_{C}$ a descontinuidade na banda de condução provocada pela junção de materiais com valores distintos de energia de banda proibida, e $V(x)$ é o termo de Hartree devido ao potencial eletrostático. Para garantir a correção do formalismo, também foi incluído o termo de correlação e troca $V_{x c}(x)[58,59]$

$$
V_{x c}(x)=\frac{-q^{2}}{\varepsilon(x) a_{0}^{*}(x) \pi \alpha r_{S}(x)}\left[1+0,7734 \ln \left(1+X(x)^{-1}\right)\right]
$$

onde

$$
\alpha=\left(\frac{4}{9 \pi}\right)^{\frac{1}{3}}, \quad r_{S}(x)=\left[\frac{4 \pi\left(a_{0}^{*}(x)\right)^{3} n(x)}{3}\right]^{\frac{-1}{3}}, \quad X(x)=\frac{r_{S}(x)}{21}, \quad a_{0}^{*}(x)=\frac{4 \hbar^{2} \pi \varepsilon(x)}{m^{*} q^{2}}
$$

no qual $a_{0}^{*}$ é o raio de Bohr efetivo, $n(x)$ é a densidade eletrônica, vide Eq.(2.12) e $\varepsilon(x)$ é a permeabilidade elétrica dos materiais [60]. Em casos que tem-se o "strain"causado pelo descasamento de rede, como por exemplo, devido a inserção de uma camada pseudomórfica de InGaAs na heterojunção $A l_{1-x} G a_{x} A s / G a A s$ pode ser levado em conta através do último termo do lado direito da Eq.(2.2). Neste termo, $C_{1}$ é o potencial de deformação na banda de condução e $\epsilon_{x x}$, $\epsilon_{y y}$ e $\epsilon_{z z}$ são componentes de "strain"[50].

A equação de Poisson, a qual produz o termo de Hartree, $V(x)$, é dada por

$$
\frac{d}{d x}\left(\kappa(x) \frac{d}{d x}\right) V(x)=\frac{\rho(x)}{\varepsilon_{0}}
$$

a qual leva em conta a variação da constante dielétrica do semicondutor $\kappa(x)$ em termos da posição. Por outro lado, a deposição de dopantes doadores na estrutura cristalina de um semi- 
condutor ocasiona o aparecimento de níveis de energia permitidos ao elétron na banda de energias proibidas. Esses níveis doadores correspondem a estados eletrônicos localizados na banda proibida. A densidade de doadores ionizados é dada por

$$
N_{d}^{+}(x)=\frac{N_{d}(x)}{1+g \cdot e^{\left(E_{F}-E_{d}\right) / k_{B} T}},
$$

onde $N_{d}(x)$ é a densidade de impurezas doadoras, $g$ é a degenerescência do nível doador, devido ao spin, portanto de valor $2, E_{d}$ a energia do nível doador, $E_{F}$ é a energia do nível de Fermi e $k_{B}$ é a constante de Boltzmann. A densidade de elétrons pode ser encontrada através da relação

$$
n_{i}=\int_{0}^{\infty} g(E) f(E) d E
$$

onde $g(E)$ é a densidade de estados do sistema considerado e $f(E)$ representa a função distribuição de Fermi-Dirac,

$$
f(E)=\frac{1}{1+\exp \left[\left(E-E_{F}\right) / k_{B} T\right]}
$$

para um sistema 2DEG, tem-se a densidade de estados

$$
g(E)=\frac{m^{*}}{\pi \hbar^{2}}
$$

substituindo as Eqs.(2.8) e (2.9) na Eq.(2.7), tem-se

$$
n_{i}=\frac{m^{*}}{\pi \hbar^{2}} \int_{E_{i}}^{\infty} \frac{1}{1+\exp \left[\left(E-E_{F}\right) / k_{B} T\right]} d E
$$

Para estruturas quantizadas uma vez que as sub-bandas já tenham sido determinadas, a distribuição de densidade de elétrons livres $n(x)$ pode ser calculada através da expressão,

$$
n(x)=\sum_{i} \Psi_{i}^{*}(x) \Psi_{i}(x) n_{i}
$$

substituindo a Eq.(2.10) na Eq.(2.11), tem-se

$$
n(x)=\frac{m^{*}}{\pi \hbar^{2}} k_{B} T \sum_{i}\left|\Psi_{i}(x)\right|^{2} \ln \left[1+\exp \left(\left(E_{F}-E_{i}\right) / k_{B} T\right)\right]
$$

em que $E_{F}$ é a energia de Fermi, a qual é considerada constante por todo o sistema na condição de equilíbrio, e tomada igual à zero. Assim, a densidade de carga pode então ser escrita como

$$
\rho(x)=-q\left(N_{d}^{+}(x)-N_{a}^{-}(x)-n(x)+p(x)\right) .
$$


onde $q$ é a carga elementar do elétron, $N_{d}^{+}(x)$ é a densidade de doadores ionizados, dada pela Eq.(2.6), $N_{a}^{-}(x)$ é a densidade não-intencional de aceitadores ionizados, $n(x)$ é a densidade de elétrons livres na banda de condução, dada pela Eq.(2.11), e $p(x)$ a densidade de lacunas livres. Nos dispositivos de interesse nesse trabalho e de acordo com a Eq.(2.13), despreza-se a densidade de carga de lacunas livres $p(x)$, uma vez que nos semicondutores tipo-n, $p(x)$ é muito pequeno quando comparada com as outras quantidades envolvidas. Observe que a energia na i-ésima sub-banda $E_{i}$, e as respectivas funções de onda $\Psi_{i}(x)$, que descrevem os efeitos do potencial do sistema sobre os elétrons são obtidas resolvendo-se a Eq.(2.1). Portanto, as equações de Schrödinger e Poisson passam a formar um sistema de equações diferenciais acopladas, que devem ser resolvidas de forma auto-consistente.

Com o desenvolvimento da solução auto-consistente de Schrödinger-Poisson unidimensional apresentada nessa Seção, pode-se estudar diversos tipos de dispositivos envolvendo heteroestruturas semicondutoras como, diodo de tunelamento ressonante (RTD) [61] e [62], estruturas tipo HEMT (High-Electron Mobility Transistor), dentre outros [50]-[52]. Para isso precisamos descrever as condições de contorno das tais estruturas de interesse, conforme desenvolvido detalhadamente na sub-seção (2.1.1).

\subsubsection{Condições de contorno}

Na Fig.(2.1), observa-se um contato Schottky depositado sobre uma sequência de camadas semicondutoras. A energia potencial na porta, na posição da barreira Schottky, estabelece uma condição de contorno, $-q V(z=0)=q \phi_{B}$, onde $q \phi_{B}$ é a altura da barreira de potencial. Porém, para sistemas submetidos a um potencial elétrico externo, a condição de contorno tornase $-q V(z=0)=q \phi_{B}-q V_{g s}$.

Por outro lado, no corpo do semicondutor ("bulk"), a energia potencial deve ter o valor da energia da banda de condução, tomada com relação ao nível de Fermi. De fato, a posição da banda de condução com respeito ao nível de Fermi no corpo do semicondutor, $E_{C}-E_{F}$, está intrinsicamente relacionada com a densidade de dopagem não-intencional de aceitadores no GaAs e pode ser calculada [63] a partir da concentração de lacunas na banda de valência:

$$
p_{0}=N_{V} \exp \left(-\frac{\left(E_{F}-E_{V}\right)}{k_{B} T}\right)
$$

Neste caso, $p_{0} \simeq N_{a}$, com 


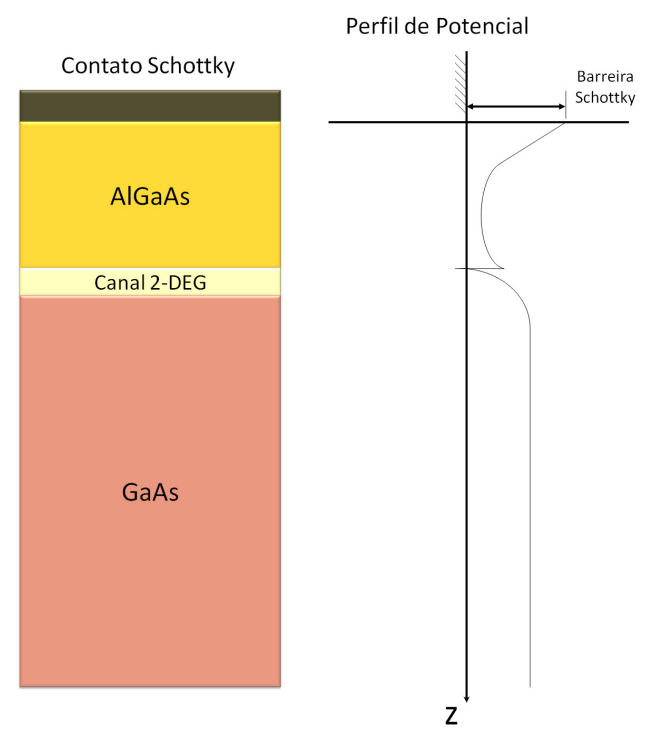

Figura 2.1: Representação esquemática de um contato Schottky e uma sequência de camadas semicondutoras, que compõem um HEMT, para exemplificar o estudo das condições de contorno. À esquerda tem-se a estrutura de camadas, e à direita, o perfil de potencial para esta estrutura. Nela é possível ver a formação de um canal condutor de elétrons entre as camadas de AlGaAs e GaAs.

$$
N_{V}=2\left(\frac{2 \pi m_{l}^{*} k_{B} T}{\hbar^{2}}\right)
$$

a densidade de estados efetiva da banda de valência, e $m_{l}^{*}$ a massa efetiva das lacunas no GaAs [64]. A Eq.(2.14) pode ser reescrita como

$$
E_{F}-E_{V}=k_{B} T \ln \left(\frac{N_{a}}{N_{V}}\right)
$$

Por outro lado, sabe-se que a energia de banda proibida, $E_{g}$, é dada por

$$
E_{g}=E_{C}-E_{F}
$$

Sendo assim, somando-se as Eqs.(2.16) e (2.17) chega-se ao resultado

$$
-q V\left(z_{b u l k}\right)=E_{C}-E_{F}=E_{g}-k_{B} T \ln \left(\frac{N_{a}}{N_{V}}\right)
$$

Finalmente, a condição de contorno para a função de onda, estabelece que esta deve ser zero tanto no substrato quanto na barreira Schottky, que é, portanto, tomada infinita para efeitos de solução da equação de Schrödinger. 
Por intermédio das condições de contorno da estrutura de interesse, podemos agora passar a descrever o método Split-Operator que será descrito detalhadamente na sub-seção (2.1.2).

\subsubsection{Método Split-Operator}

Vamos agora resolver as Eqs.(2.1) e (2.5) seguindo o método de diferenças não-uniforme (MDFNU), que é apresentado detalhadamente no Apêndice A, salientando que o uso de variáveis não-uniforme requer um tratamento mais cuidadoso que o caso convencional da discretização uniforme, uma vez que a discretização não-uniforme destrói a simetria do operador Hamiltoniano, tornando-se muito mais difícil calcular $\Psi$. Como alternativa a este problema, foi introduzido em 1982 por Fleit e Fleck [65] um esquema de propagação bastante eficiente e preciso para se resolver a equação de Schrödinger. A técnica de split-operator (SO) [66], que visa encontrar os auto-estados da equação de Schrödinger, foi proposta originalmente para se determinar modos de guias de onda ópticos, onde as equações são muito similares à equação de Schrödinger. Este método foi bem sucedidamente aplicado à problemas de mecânica quântica, e tem por base a equação de Schrödinger dependente do tempo

$$
i \hbar \frac{\partial \Psi(\vec{r}, t)}{\partial t}=H \Psi(\vec{r}, t)
$$

A função de onda é obtida como usual

$$
\Psi(\vec{r}, t+d t)=\exp \left(\frac{-i}{\hbar} \int_{t}^{t+d t} H d t\right) \Psi(\vec{r}, t)
$$

Sendo assim, como o Hamiltoniano é função do tempo a Eq.(2.20) pode ser escrita como

$$
\Psi(\vec{r}, t+d t)=\exp \left(-\frac{i H d t}{\hbar}\right) \Psi(\vec{r}, t)
$$

O Hamiltoniano que aparece na Eq.(2.20) pode ser reescrito muito simplesmente da forma

$$
H=\frac{p^{2}}{2 m}+V(\vec{r})
$$

Então, 


$$
\Psi(\vec{r}, t+d t)=\exp \left[-\frac{i d t}{\mathrm{~h}}\left(\frac{V(\vec{r})}{2}+\frac{p^{2}}{2 m}+\frac{V(\vec{r})}{2}\right)\right] \Psi(\vec{r}, t)
$$

O método de SO o qual foi detalhado na referência Ref. [66] consiste em "quebrar" o operador exponencial da forma mostrada abaixo

$$
\Psi(\vec{r}, t+d t)=\exp \left(-\frac{i d t V(\vec{r})}{2 \hbar}\right) \exp \left(-\frac{i d t p^{2}}{2 m \hbar}\right) \exp \left(-\frac{i d t V(\vec{r})}{2 \hbar}\right) \Psi(\vec{r}, t)
$$

o que pode ser considerado adequado se algumas condições forem levadas em conta:

$$
e^{A+B+A}=e^{A} e^{B} e^{A} \Longleftrightarrow[A,[A, B]]=[B,[A, B]]=0
$$

O erro introduzido nesta expressão é devido à não comutatividade dos operadores energia cinética e energia potencial, da ordem $\left(d t^{3}\right)$ [66]. Observe que, uma vez que na Eq. (2.24) cada operador é unitário, a norma deve ser conservada. O procedimento é semelhante ao proposto por Degani et al [66] para o caso do formalismo de confinamento unidimensional. Assim, chamamos

$$
\xi(\vec{r}, t+d t)=\exp \left(-\frac{i d t V(\vec{r})}{2 \hbar}\right) \Psi(\vec{r}, 0)
$$

em que $\Psi(\vec{r}, 0)$ a função de onda inicial e $V(\vec{r})$ são escolhas tentativas. Aplicando-se o operador exponencial a direita da Eq. $(2.24)$ na $\xi(\vec{r}, t+d t)$, pode-se escrever

$$
\eta(\vec{r}, t+d t)=\exp \left(-\frac{i d t p^{2}}{2 m \hbar}\right) \xi(\vec{r}, t+d t)
$$

Para evitar uma normalização constante [67], encontrou-se outro operador, unitário, que tenha a mesma expansão que este até a maior ordem possível, considerando que o operador exponencial pode ser expandido como, onde $\kappa=-\frac{i d t p^{2}}{2 m \hbar}$

$$
\frac{1+\frac{\kappa}{2}}{1-\frac{\kappa}{2}}=\left(1+\frac{\kappa}{2}\right)\left(1-\frac{\kappa}{2}\right)^{-1}
$$

Expandindo a parcela $\left(1-\frac{\kappa}{2}\right)^{-1}$, temos

$$
\frac{1+\frac{\kappa}{2}}{1-\frac{\kappa}{2}}=\left(1+\frac{\kappa}{2}\right)\left[1+(-1)\left(-\frac{\kappa}{2}\right)+\frac{(-1)(-2)}{2 !}\left(\frac{-\kappa}{2}\right)^{2}+\frac{(-1)(-2)(-3)}{3 !}\left(\frac{-\kappa}{2}\right)^{3}+\ldots\right]
$$


no qual podemos escrever a Eq.(2.29)

$$
\frac{1+\frac{\kappa}{2}}{1-\frac{\kappa}{2}}=1+\kappa+\frac{\kappa^{2}}{2}+\ldots
$$

onde a Eq.(2.30) pode ser igualada a

$$
e^{\kappa}=1+\kappa+\frac{\kappa^{2}}{2 !}+\frac{\kappa^{3}}{3 !}+\ldots
$$

implicando assim, um erro proporcional a $\left(d t^{2}\right)$ [67]. Sabendo que

$$
\frac{1+\frac{\kappa}{2}}{1-\frac{\kappa}{2}}=e^{\kappa}
$$

substituindo a Eq.(2.32) na Eq.(2.27), tem-se

$$
\left(1-\frac{i d t p^{2}}{4 m \hbar}\right)=\frac{\eta(\vec{r}, t+d t)}{\xi(\vec{r}, t+d t)}\left(1+\frac{i d t p^{2}}{4 m \hbar}\right)
$$

Rearranjando a Eq.(2.33)

$$
\eta(\vec{r}, t+d t)\left(1+\frac{i d t p^{2}}{4 m \hbar}\right)=\left(1-\frac{i d t p^{2}}{4 m \hbar}\right) \xi(\vec{r}, t+d t)
$$

onde $p=-i \hbar \frac{\partial}{\partial x}$

$$
\eta(\vec{r}, t+d t)\left[1+\frac{i d t}{4 m \hbar}\left(-i \mathrm{\hbar} \frac{\partial}{\partial x}\right)^{2}\right]=\left[1-\frac{i d t}{4 m \hbar}\left(-i \mathrm{\hbar} \frac{\partial}{\partial x}\right)^{2}\right] \xi(\vec{r}, t+d t)
$$

manipulando a Eq.(2.35), obtem-se

$$
\eta(\vec{r}, t+d t)\left[1-\frac{i \hbar d t}{4 m}\left(\frac{\partial^{2}}{\partial x^{2}}\right)\right]=\left[1+\frac{i \hbar d t}{4 m}\left(\frac{\partial^{2}}{\partial x^{2}}\right)\right] \xi(\vec{r}, t+d t)
$$

chamando $\epsilon=\frac{i \hbar d t}{2 m}$, tem-se

$$
\eta(\vec{r}, t+d t)\left[1-\frac{\epsilon}{2}\left(\frac{\partial^{2}}{\partial x^{2}}\right)\right]=\left[1+\frac{\epsilon}{2}\left(\frac{\partial^{2}}{\partial x^{2}}\right)\right] \xi(\vec{r}, t+d t)
$$

A derivada segunda no esquema de diferenças finita não-uniforme pode ser escrita como

$$
f_{i}^{\prime \prime}=\frac{2 f_{i-1}}{\Delta_{i-1}\left(\Delta_{i-1}+\Delta_{i}\right)}-\frac{2 f_{i}}{\Delta_{i-1} \Delta_{i}}+\frac{2 f_{i+1}}{\Delta_{i}\left(\Delta_{i-1}+\Delta_{i}\right)}
$$

Substituindo o esquema da Eq.(2.38) na Eq.(2.37) encontramos 


$$
\begin{aligned}
\eta_{i}-\frac{\epsilon}{2} \frac{\partial^{2} \eta_{i}}{\partial x^{2}} & =\eta_{i}-\frac{\epsilon}{2}\left(\frac{2 \eta_{i-1}}{\Delta_{i-1}\left(\Delta_{i-1}+\Delta_{i}\right)}-\frac{2 \eta_{i}}{\Delta_{i-1} \Delta_{i}}+\frac{2 \eta_{i+1}}{\Delta_{i}\left(\Delta_{i-1}+\Delta_{i}\right)}\right)= \\
& =\xi_{i}+\frac{\epsilon}{2} \frac{\partial^{2} \xi_{i}}{\partial x^{2}}=\xi_{i}+\frac{\epsilon}{2}\left(\frac{2 \xi_{i-1}}{\Delta_{i-1}\left(\Delta_{i-1}+\Delta_{i}\right)}-\frac{2 \xi_{i}}{\Delta_{i-1} \Delta_{i}}+\frac{2 \xi_{i+1}}{\Delta_{i}\left(\Delta_{i-1}+\Delta_{i}\right)}\right)
\end{aligned}
$$

Chamando

$$
-\Theta_{i}=\xi_{i}+\frac{\epsilon}{2}\left(\begin{array}{c}
\frac{2 \xi_{i-1}}{\Delta_{i-1}\left(\Delta_{i-1}+\Delta_{i}\right)} \\
-\frac{2 \xi_{i}}{\Delta_{i-1} \Delta_{i}}+\frac{2 \xi_{i+1}}{\Delta_{i}\left(\Delta_{i-1}+\Delta_{i}\right)}
\end{array}\right)
$$

A Eq.(2.39) resulta

$$
\eta_{i}-\frac{\epsilon}{2}\left(\begin{array}{c}
\frac{2 \eta_{i-1}}{\Delta_{i-1}\left(\Delta_{i-1}+\Delta_{i}\right)} \\
-\frac{2 \eta_{i}}{\Delta_{i-1} \Delta_{i}}+\frac{2 \eta_{i+1}}{\Delta_{i}\left(\Delta_{i-1}+\Delta_{i}\right)}
\end{array}\right)=-\Theta_{i}
$$

Manipulando a Eq.(2.41)

$$
\eta_{i}-\frac{\eta_{i-1}}{\Delta_{i-1}\left(\Delta_{i-1}+\Delta_{i}\right)} \epsilon+\frac{\eta_{i}}{\Delta_{i-1} \Delta_{i}} \epsilon-\frac{\eta_{i+1}}{\Delta_{i}\left(\Delta_{i-1}+\Delta_{i}\right)} \epsilon=-\Theta_{i}
$$

Simplificando a Eq.(2.42)

$$
\frac{\epsilon}{\Delta_{i}\left(\Delta_{i-1}+\Delta_{i}\right)} \eta_{i+1}-\left(1+\frac{\epsilon}{\Delta_{i-1} \Delta_{i}}\right) \eta_{i}+\frac{\epsilon}{\Delta_{i-1}\left(\Delta_{i-1}+\Delta_{i}\right)} \eta_{i-1}=\Theta_{i}
$$

a qual pode ser escrita na forma de uma equação matricial,

$$
\sum_{j} M_{i, j} \eta_{j}=\Theta_{j}
$$

onde

$$
M_{i, j}=\left\{\begin{array}{cc}
-\left(1+\frac{\epsilon}{\Delta_{i-1} \Delta_{i}}\right) & , \text { se } j=i \\
\frac{\epsilon}{\Delta_{i}\left(\Delta_{i-1}+\Delta_{i}\right)} & , \text { se } j=i+1 \\
\frac{\epsilon}{\Delta_{i-1}\left(\Delta_{i-1}+\Delta_{i}\right)} & , \text { se } j=i-1 \\
0 \quad, \text { outros valores de } j
\end{array}\right.
$$

em que $M_{i, j}$ é uma matriz tridiagonal e pode ser resolvida usando-se o conhecido algoritmo de Thomas [68]. Analogamente, aplica-se o operador

$$
\Psi(\vec{r}, t+d t)=\exp \left(-\frac{i d t V(\vec{r})}{2 \hbar}\right) \eta(\vec{r}, t+d t)
$$


Tendo $\eta(\vec{r}, t+d t)$, basta então multiplicá-lo pela $\exp \left(-\frac{i d t V(\vec{r})}{2 \hbar}\right)$ que se obtem $\Psi(\vec{r}, t+d t)$ que é a função de onda num instante $t+d t$. Tendo-se tal função no instante anterior está determinada a evolução temporal de $\Psi(t)$. Ao contrário da propagação em tempo real, a propagação em tempo imaginário exige que as funções de onda sejam normalizadas a cada evolução temporal $\Delta t=-i \tau$, e a função de onda sempre evolui para o estado fundamental do sistema descrito pelo Hamiltoniano $\mathbf{H}$ independente do tempo [67]. Vamos então normalizar as funções de onda e ortagonalizá-las. A normalização é obtida através da definição

$$
|\tilde{\Psi}\rangle=\frac{|\Psi\rangle}{\sqrt{\langle\Psi \mid \Psi\rangle}}
$$

onde $\sqrt{\langle\Psi \mid \Psi\rangle}$ é a norma de $|\Psi\rangle$. Assim sendo, vamos calcular a norma segundo a definição

$$
\langle\Psi \mid \Psi\rangle=\int \Psi^{*}(x) \Psi(x) d x
$$

Obtida a normalização, devemos impor a ortogonalidade das auto-funções através do método de Gram-Schmidt, em que

$$
\Psi_{i}^{\perp}(x, t)=\Psi_{i}(x, t)-\sum_{j<i}\left\langle\Psi_{j}^{\perp}(x, t) \mid \Psi_{i}(x, t)\right\rangle \Psi_{j}^{\perp}(x, t)
$$

Sendo assim, os auto-estados da equação de Schrödinger podem agora ser encontrados, bastando-se conhecer as condições de contorno que a função de onda deve satisfazer.

Resumindo no fluxograma apresentado na Fig.(2.2) o procedimento numérico empregado para a solução do problema auto-consistente.

\subsubsection{Estruturas de poços quânticos investigadas}

O formalismo desenvolvido na Seção (2.1), já foi bem sucedidamente demonstrado para heteroestruturas semicondutoras, Ref. [50]. Neste trabalho esse formalismo é aferido com a investigação de dispositivos da família dos HEMTs. A primeira estrutura a ser investigada é uma heterojunção de $G a A s / A l_{0,3} G a_{0,7} A s$ com dopagem modulada, que foi proposta por Tan et al, na Ref. [47]. A estrutura utilizada é mostrada na Fig.(2.3), onde a heteroestrutura foi crescida sobre um substrato de GaAs. Especificamente, uma camada de GaAs com $5000 \AA$ de espessura foi crescida sobre o substrato, seguida por uma fina camada de AlGaAs intrínseco de $50 \AA$ de espessura, uma camada de AlGaAs com $N_{D}=1 \times 10^{18} \mathrm{~cm}^{-3}$ e espessura de $200 \AA$ e por fim, uma camada de GaAs com $N_{D}=1 \times 10^{18} \mathrm{~cm}^{-3}$ e espessura de $150 \AA$. O método numérico 


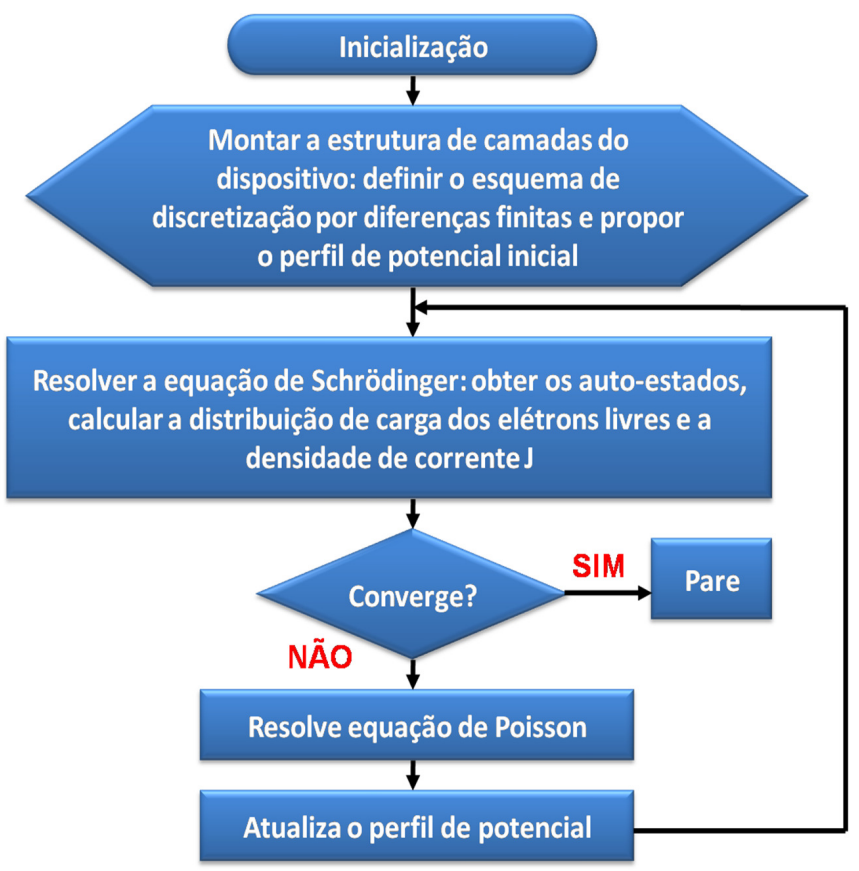

Figura 2.2: Fluxograma representando o procedimento numérico empregado para a solução do problema auto-consistente.

apresentado na Seção (2.1) foi utilizado para contrastar sua solução com os resultados obtidos na Ref. [47]. Com isso, pode-se construir o perfil de banda de condução para a heteroestrutura, mostrada na Fig.(2.3), esquematizada na Fig.(2.4). Adicionalmente, a densidade eletrônica, também pode ser encontrada e é mostrada na Fig.(2.5).

De posse, dos resultados obtidos nas Figs.(2.4) e (2.5), pode-se verificar a validade da solução numérica auto-consistente de Schrödinger e Poisson, pois, os resultados aqui obtidos estão em pleno acordo com o trabalho de Tan et al, na Ref. [47].

Outra estrutura a ser discutida neste trabalho são as heterojunções de um transistor de alta mobilidade eletrônica - HEMT (High electron mobility transistor) convencional de AlGaAs/GaAs, proposta por B. Vinter, Ref. [69], que propôs num trabalho pioneiro a solução auto-consistente das equações de Schrödinger e Poisson em dispositivos de heterojunção. Os nossos resultados são contrastados com o trabalho de Vinter [69], comprovando assim a sua validade. A Fig.(2.6) representa a estrutura em questão, que apresenta uma camada de $A l_{x} G a_{1-x} A s$ dopada do tipo-n de espessura $d_{2}$ com uma concentração de doadores $N_{D}=1,3 \times 10^{18} \mathrm{~cm}^{-3}$, 


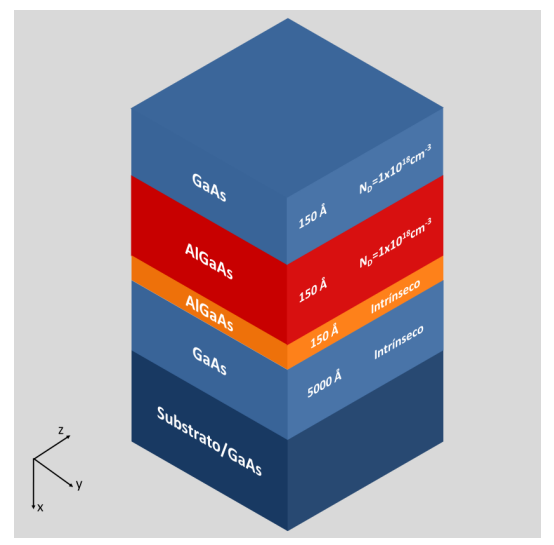

Figura 2.3: Diagrama esquemático da estrutura de camadas da heteroestrutura semicondutora de dopagem modulada GaAs $/ \mathrm{Al}_{0,3} \mathrm{Ga}_{0,7}$ As.

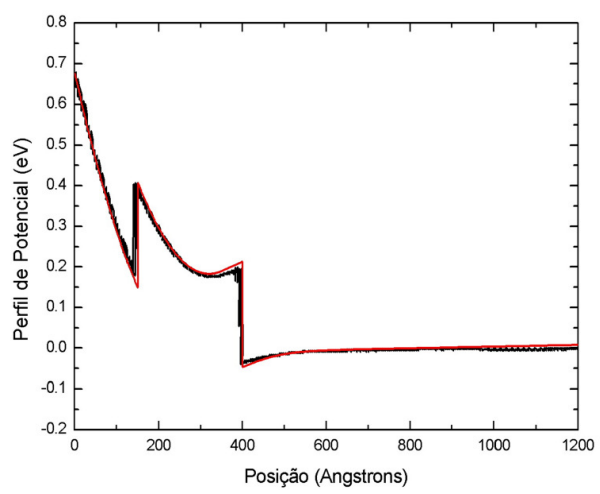

Figura 2.4: Perfil de potencial de condução em eV em função da posição na heteroestrutura GaAs $/ \mathrm{Al}_{0,3} \mathrm{Ga}_{0,7} \mathrm{As}$, onde a linha vermelha representa o trabalho desenvolvido e a linha preta o trabalho proposto por Tan et al, na Ref. [47].

seguida por uma camada de $A l_{x} G a_{1-x} A s$ não-dopada de espessura $d_{i}$ e uma camada de GaAs não-dopada de espessura $d_{1}$ crescida sobre um substrato semi-isolante de GaAs. Os demais parâmetros utilizados na solução numérica encontra-se na Tabela (2.1).

Com o auxílio do método numérico discutido anteriormente, pode-se obter os resultados para o perfil de banda de condução considerando duas tensões distintas, $V_{g s}=-1,5 \mathrm{~V}$ e $V_{g s}=$ $-0,5 \mathrm{~V}$. Os resultados mostrados nas Figs.(2.7-a) e (2.7-b), estão em pleno acordo com os resultados obtidos por Vinter na Ref. [69] para uma temperatura de $300 \mathrm{~K}$.

Comparando as Figs.(2.8-a) e (2.8-b) pode-se verificar que para tensões de porta muito negativas, neste caso $V_{g s}=-1,5 \mathrm{~V}$, a camada de $A l G a A s$ praticamente não apresentará elétrons livres, enquanto que, tensões um pouco maiores, neste caso $V g s=-0,5 V$, verifica-se a presença 


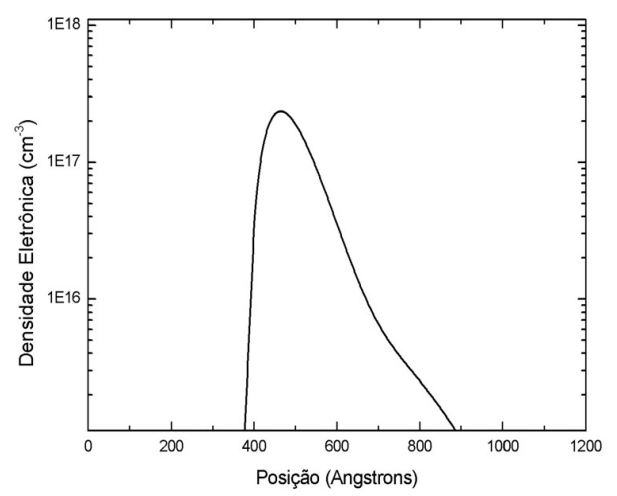

Figura 2.5: População eletrônica por unidade de volume em função da posição na heteroestrutura de GaAs $/ \mathrm{Al}_{0,3} \mathrm{Ga}_{0,7} \mathrm{As}$, proposta por Tan et al, na Ref. [47].

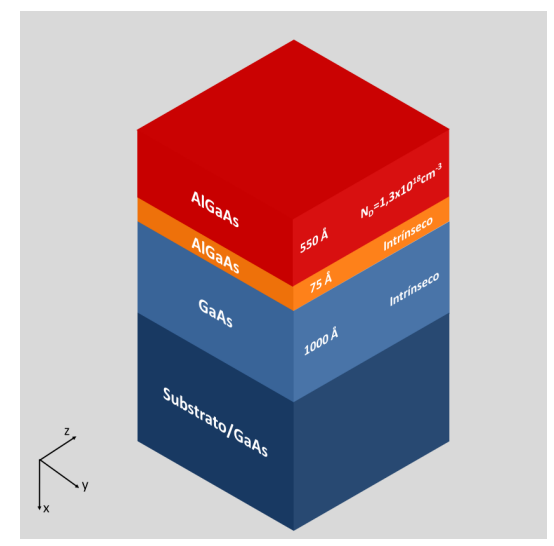

Figura 2.6: Diagrama esquemático da estrutura de camadas das heteroestrutura semicondutoras que constituem o HEMT.

de muitos elétrons livres, podendo ocorrer uma corrente parasita significativa ao longo da camada de AlGaAs, o que degrada o desempenho deste dispositivo.

\subsection{Solução de sistemas envolvendo fios quânticos}

A partir dos cálculos desenvolvidos na Seção (2.1), pretende-se fazer uma extensão da solução de poços quânticos para a solução de sistemas envolvendo fios quânticos.

Agora é necessário fazer todas as extensões corretas das equações unidimensionais descritas na Seção (2.1), para as equações bidimensionais. Sendo assim, considere a equação bidimensional de Schrödinger independente do tempo, 


\begin{tabular}{|c|c|c|}
\hline$\Delta E_{C}$ & Descontinuidade na banda de condução $(\mathrm{meV})$ & 260 \\
\hline$E_{B}$ & Energia do nível doador $(\mathrm{meV})$ & 50 \\
\hline$x$ & Concentração de $\mathrm{Al}$ no AlGaAs & 0,26 \\
\hline$d_{2}$ & Espessura de AlGaAs dopado $(\AA)$ & 550 \\
\hline$d_{i}$ & Espessura de AlGaAs não-dopado $(\AA)$ & 75 \\
\hline$d_{1}$ & Espessura de GaAs não-dopado $(\mu \mathrm{m})$ & 1 \\
\hline$N_{D}$ & Densidade de doadores $\left(10^{18} \mathrm{~cm}^{-3}\right)$ & 1,3 \\
\hline
\end{tabular}

Tabela 2.1: Parâmetros usados no dispositivo estudado por Vinter
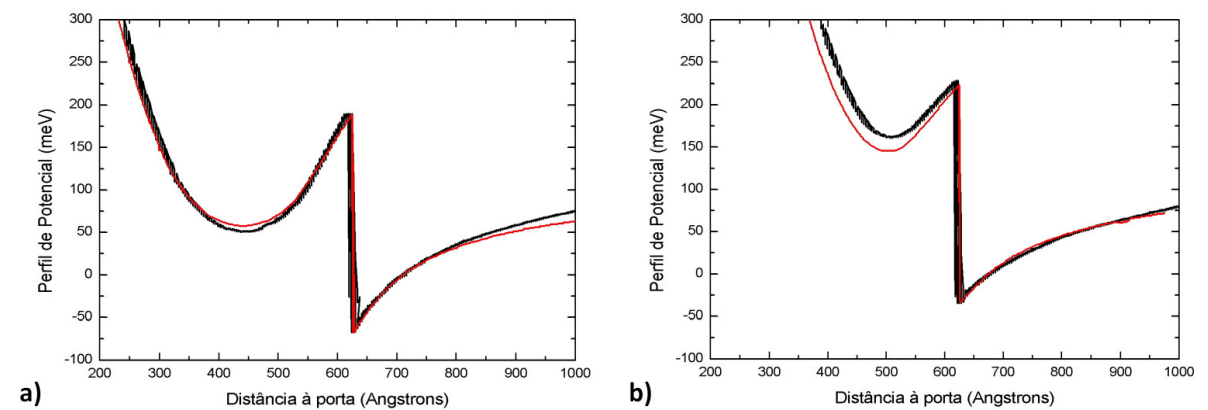

Figura 2.7: Perfil de banda de condução em meV em função da distância à porta, para as tensões: a) $V_{g s}=-0,5 \mathrm{~V}$ e b) $V_{g s}=-1,5 \mathrm{~V}$, à uma temperatura de $300 \mathrm{~K}$, onde a linha vermelha representa o trabalho desenvolvido aqui e a linha preta representa o trabalho de Vinter na Ref. [69].

$$
\begin{gathered}
-\frac{\hbar^{2}}{2 a(x, y)} \frac{d}{d x}\left(\frac{a^{2}(x, y)}{m^{*}(x, y)} \frac{d}{d x} \frac{1}{a(x, y)}\right) \Psi(x, y)-\frac{\hbar^{2}}{2 a(x, y)} \frac{d}{d y}\left(\frac{a^{2}(x, y)}{m^{*}(x, y)} \frac{d}{d y} \frac{1}{a(x, y)}\right) \Psi(x, y)+ \\
+V_{e f}(x, y) \Psi(x, y)=E \Psi(x, y)
\end{gathered}
$$

na qual empregaremos o esquema de diferenças-finitas não uniforme nas direções $x$ e $y$ para calcular as sub-bandas de energia e auto-funções do sistema bidimensional. Na Eq.(2.47), $x$ é a direção perpendicular a camada epitaxial, e a direção y é a direção entre contatos, como mostrado na Fig.(2.9). Observe que a Eq.(2.47), assim como a Eq.(2.1), também leva em conta a dependência da massa efetiva e da constante de rede [50] no operador energia cinética. $\mathrm{O}$ potencial efetivo $V_{e f}$ é dado por

$$
V_{e f}(x, y)=\Delta E_{C}(x, y)-q V(x, y)+C_{1}\left(\epsilon_{x x}+\epsilon_{y y}+\epsilon_{z z}\right)+V_{x c}(x, y)
$$

Analogamente, no formalismo bidimensional a equação de Poisson, a qual produz o 

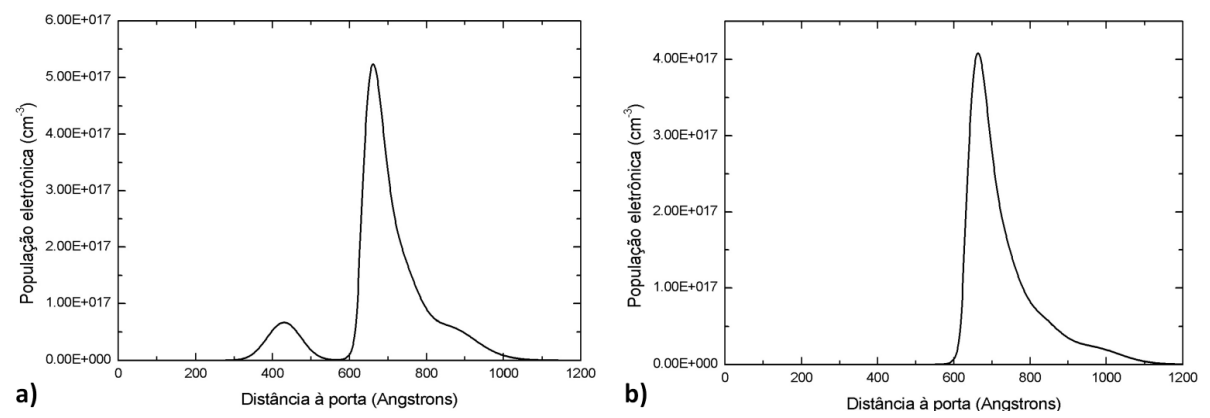

Figura 2.8: População eletrônica por unidade de volume em função da posição em relação à porta, para as tensões: a) $V_{g s}=-0,5 \mathrm{~V}$ e b) $V_{g s}=-1,5 \mathrm{~V}$.

a)

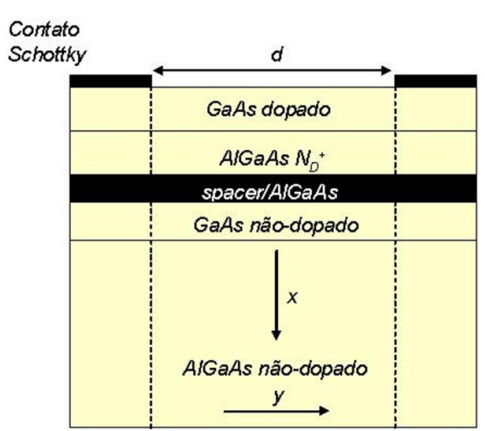

b)

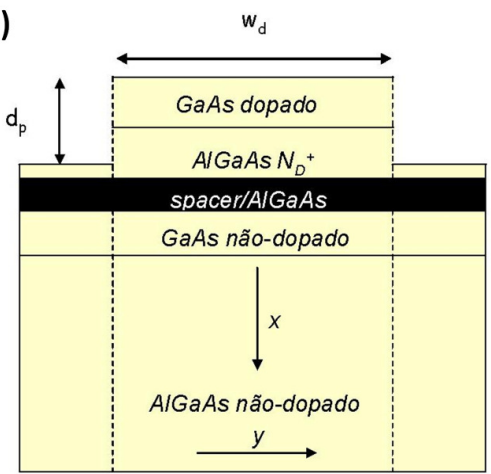

Figura 2.9: Diagrama esquemático de transistores de fios quânticos sobre uma heteroestrutura convencional AlGaAs/GaAs com uma estrutura: a) de split-gate e b) corrosão provocando o confinamento eletrônico.

termo de Hartree, $V(x, y)$ da Eq.(2.48), é dada por

$\frac{d}{d x}\left(\varepsilon_{0} \kappa(x, y) \frac{d}{d x}\right) V(x, y)+\frac{d}{d y}\left(\varepsilon_{0} \kappa(x, y) \frac{d}{d y}\right) V(x, y)=-\frac{q}{\epsilon_{0}}\left(N_{d}^{+}(x, y)-N_{a}^{-}(x, y)-n(x, y)\right)$

Com isso, pode-se escrever a densidade eletrônica $n(x, y)$ em termos das funções de onda eletrônicas $\Psi(x, y)[13]$,

$$
n(x, y)=\sum_{k=1}^{m} \Psi_{k}^{*}(x, y) \Psi_{k}(x, y) \int_{E_{k}}^{\infty} \frac{\sqrt{2 m^{*}}}{\pi \hbar \sqrt{E-E_{k}}} \frac{d E}{\left[1+\exp \left(\left(E-E_{k}\right) / k_{B} T\right)\right]}
$$

onde $m^{*}$ é a massa efetiva do elétron no canal 1-DEG, $k_{B}$ é a constante de Boltzmann, $T$ é a temperatura absoluta, $E_{F}$ é o nível da energia de Fermi e $E_{k}$ representa o $k$-ésimo auto-valor de energia. A soma é realizada sobre todas as sub-bandas $k$ 's. 
O nível de Fermi $E_{F}$ é assumido ser o zero de energia, e a condição usual de neutralidade de carga é levada em conta na formulação acima. Vale também observar que, as condições de contorno na função de onda estabelecem que esta deva ser zero tanto no substrato quanto na barreira Schottky. Sendo conhecidas as condições de contorno, a solução auto-consistente das Eqs.(2.47) e (2.49) fornece a distribuição de carga e todas as demais quantidades de interesse.

Para efeitos práticos, uma das quantidades mais importantes para determinação do desempenho do dispositivo, é a densidade eletrônica $n(x, y)$, dada pela Eq.(2.50). A densidade de elétrons $n(x, y)$ na estrutura pode ser calculada conhecendo-se os autovalores de energia $\left(E_{k}\right)$ da equação de Schröndinger em conjunto com as funções de onda $\left(\Psi_{k}(x, y)\right)$, como mostrado na Eq.(2.50), em que a soma deve ser levada em conta sobre todas as sub-bandas $k$ consideradas relevantes no problema.

Fazendo-se uma mudança de variáveis na Eq.(2.50) e chamando

$$
x=\frac{E-E_{k}}{k_{B} T}
$$

a integral da Eq.(2.50) pode ser reescrita como

$$
\begin{gathered}
\int_{E_{k}}^{\infty} \frac{1}{\sqrt{E-E_{k}}} \frac{d E}{\left[1+\exp \left(\frac{E-E_{k}}{k_{B} T}\right)\right]}=\int_{a=\frac{E-E_{k}}{k_{B} T}}^{\infty} \frac{1}{\sqrt{k_{B} T x}} \frac{k_{B} T d x}{1+e^{x}} \\
=\sqrt{k_{B} T} \int_{a}^{\infty} \frac{1}{\sqrt{x}} \frac{d x}{1+e^{x}}
\end{gathered}
$$

Substituindo a Eq.(2.52) na Eq.(2.50) obtém-se

$$
n(x, y)=\frac{\sqrt{2 m^{*} k_{B} T}}{\pi \hbar} \sum_{k=1}^{m} \Psi_{k}^{*}(x, y) \Psi_{k}(x, y) \int_{\frac{E-E_{k}}{k_{B} T}}^{\infty} \frac{1}{\sqrt{x}} \frac{d x}{1+e^{x}}
$$

Chamando $n_{k}$ a densidade eletrônica em cada sub-banda $k$

$$
n_{k}=\frac{\sqrt{2 m^{*} k_{B} T}}{\pi \hbar} \int_{a}^{\infty} \frac{1}{\sqrt{x}} \frac{d x}{1+e^{x}}
$$

a Eq.(2.50) finalmente pode ser reescrita como

$$
n(x, y)=\sum_{k=1}^{m} \Psi_{k}^{*}(x, y) \Psi_{k}(x, y) n_{k}
$$

A integral na Eq.(2.52) é frequentemente obtida usando-se a definição de integral de Fermi-Dirac completa, 


$$
F_{j}(x)=\frac{1}{\Gamma(j+1)} \int_{0}^{\infty} \frac{t^{j}}{1+\exp (t-x)} d t
$$

para algum valor tabelado. Tal procedimento foi utilizado por Trellakis et al [70] e Snider et al [47]. Há também, vários algoritmos propostos na literatura para este cálculo, dos quais empregamos aquele da Ref. [68].

\subsubsection{Desenvolvimento do cálculo da densidade eletrônica de $\mathrm{n}(\mathrm{x}, \mathrm{y})$}

Usando-se o Teorema do Valor Médio (TVM) para integrais do cálculo fundamental, tem-se que

$$
\int_{a}^{b} h(x) g(x) d x=h(\xi) \int_{a}^{b} g(x) d x
$$

para algum $\xi \in[a, b]$, com $f(x)=h(x) \cdot g(x)$, sendo $h(x)$ e $g(x)$ funções contínuas e diferenciáveis no intervalo $[a, b]$ e $g(x)$ não muda de sinal em $[a, b]$. Desta forma, pode-se escrever a integral da Eq.(2.50) como

$$
\int_{a}^{b=\infty} \frac{1}{\sqrt{x}} \frac{d x}{1+e^{x}}=\frac{1}{\sqrt{\xi}} \int_{a}^{\infty} \frac{d x}{1+e^{x}}
$$

a constante $\xi$ pode ser encontra usando-se ainda o $T V M$, aplicando-se a definição

$$
f^{\prime}(\xi)=\frac{f(b)-f(a)}{b-a}
$$

Calculando-se o lado direito da Eq.(2.59) resulta

$$
f^{\prime}(\xi)=\frac{\left[\sqrt{b}\left(1+e^{b}\right)\right]^{-1}-\left[\sqrt{a}\left(1+e^{a}\right)\right]^{-1}}{b-a}
$$

e tomando-se o limite

$$
\lim _{b \rightarrow \infty}\left[f^{\prime}(\xi)\right]=0
$$

A Eq.(2.59) torna-se

$$
f^{\prime}(\xi)=0
$$

Por outro lado, calculando-se a derivada da função f no ponto $x=\xi$ obtém-se 


$$
f^{\prime}(\xi)=\frac{-\xi^{\frac{-3}{2}}\left(1+e^{\xi}\right)^{-1}}{2}-\xi^{\frac{-1}{2}} e^{\xi}\left(1+e^{\xi}\right)^{-2}=0
$$

Como $\xi$ não é facilmente encontrada através de um procedimento analítico simples, recorremos à solução deste problema através do Método de Newton, e em poucas iterações é possível obter o valor de $\xi$ com a precisão desejada.

Assim, obtém-se

$$
\xi=0,84540
$$

e

$$
\frac{1}{\sqrt{\xi}}=1,08759
$$

A integral na Eq.(2.58) é conhecida

$$
\int_{\frac{E_{k}-E_{F}}{k_{B} T}}^{\infty} \frac{d x}{1+e^{x}}=\ln \left(1+\exp \left(\frac{E_{F}-E_{k}}{k_{B} T}\right)\right)
$$

Substituindo a Eq.(2.61) e Eq.(2.62) na Eq.(2.58) e esta na Eq.(2.54) pode-se obter a densidade eletrônica $\mathrm{n}(\mathrm{x}, \mathrm{y})$, como sendo

$$
n(x, y)=\frac{\sqrt{2 m^{*} k_{B} T}}{\pi \hbar} \frac{1}{\sqrt{\xi}} \sum_{k=1}^{m} \Psi_{k}^{*}(x, y) \Psi_{k}(x, y) \ln \left(1+\exp \left(\frac{E_{F}-E_{k}}{k_{B} T}\right)\right)
$$

reduzindo-a a um cálculo semelhante ao efetuado no formalismo unidimensional.

\subsubsection{Solução da Equação de Poisson não-linear pelo Método de Relaxação}

A equação de Poisson bidimensional foi resolvida usando-se o método de relaxação no esquema FTCS (Forward Time Centered Space), com discretização não-uniforme e empregandose a Eq.(2.38) para a derivada segunda no esquema de discretização não-uniforme, assim:

$$
\left[a_{i, j}^{x} V_{i-1, j}^{n}+b_{i, j}^{x} V_{i, j}^{n}+c_{i, j}^{x} V_{i+1, j}^{n}\right]+\left[a_{i, j}^{y} V_{i, j-1}^{n}+b_{i, j}^{y} V_{i, j}^{n}+c_{i, j}^{y} V_{i, j+1}^{n}\right]=\frac{\rho_{i, j}}{\varepsilon_{0}}
$$

Os índices $i$ e $j$ identificaram a posição na malha bidimensional, $i$ significa o índice na direção $x$ (direção de crescimento), e $j$ indica a direção $y$, e $a_{i, j}^{x}, b_{i, j}^{x}, c_{i, j}^{x} \ldots$ são os coeficientes obtidos a partir da aplicação da Eq.(2.38), na Eq.(2.37). Em seguida, introduz-se uma 
dependência artificial no tempo somente para a execução do algoritmo. A equação de Poisson discretizada é descrita como segue,

$$
V_{i, j}^{n+1}=\frac{1}{\left(b_{i, j}^{x}+b_{i, j}^{y}\right)} \times\left[\frac{\rho}{\varepsilon_{0}}\left(1+d t\left(b_{i, j}^{x}+b_{i, j}^{y}\right)\right)-B^{*}\right]
$$

com

$$
B^{*}=\left[a_{i, j}^{x} V_{i-1, j}+b_{i, j}^{x} V_{i, j}+c_{i, j}^{x} V_{i+1, j}\right]+\left[a_{i, j}^{y} V_{i, j-1}+b_{i, j}^{y} V_{i, j}+c_{i, j}^{y} V_{i, j+1}\right]
$$

e

$$
\rho=q\left[N_{d}^{+}(x, y)-N_{a}^{-}(x, y)+n(x, y)\right]
$$

Este procedimento é aplicado iterativamente para calcular a evolução no potencial, primeiramente para se obter o potencial inicial, e depois em cada passo do cálculo da função de onda na Eq.(2.24).

A condição de contorno para a equação de Poisson é dada pelo potencial de superfície, $V_{s}$, ou quando houver contatos Schottky, pela tensão aplica à porta, $V_{g}$, como mostrado na Fig.(2.10). Por outro lado, se a região considerada for o semicondutor "bulk", a condição de contorno a ser considerada é aquela dada pela posição da banda de condução com respeito ao nível de Fermi, com a presença de densidade de dopante ionizado não-intencional, $N_{a}^{-}$.

Dependendo da geometria do dispositivo, como aquela mostrada na Fig.(2.9-b) pode-se usar o esquema da Fig.(2.11) para se diminuir o esforço computacional. Vale salientar que o desenho da Fig.(2.11) não se encontra em escala, uma vez que a região tracejada do retângulo é muito menor do que as regiões laterais.

A escolha do método de relaxação para o cálculo da equação bidimensional de Poisson, deve-se do fato de se tratar de um problema de condição de contorno, e a solução deste tipo de equação diferencial é dada por funções harmônicas. Tendo em vista que o teorema de unicidade das funções harmônicas, [71] garante que há apenas uma função harmônica que satisfaça uma dada condição de contorno, tem-se que esse método é bastante adequado.

\subsubsection{Estruturas de fios quânticos investigadas}

A primeira estrutura investigada neste trabalho, utilizando a modelagem bidimensional, é aquela proposta por Snider et al [13], composta por uma estrutura de $A l G a A s / G a A s$, mostrada 


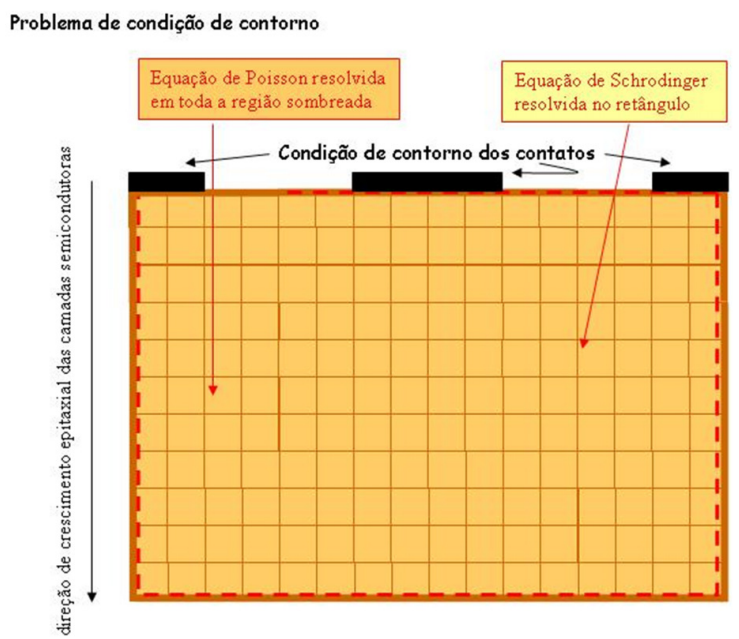

Figura 2.10: Representação esquemática da região de solução do cálculo auto-consistente das equações de Schrödinger e de Poisson. Na figura vêem-se contatos que podem polarizar a estrutura. A equação de Schrödinger e de Poisson é resolvida no interior da região marcada pelo retângulo tracejado, que estabelece também as condições de contorno, como o dispositivo mostrado na Fig. (2.9-a).

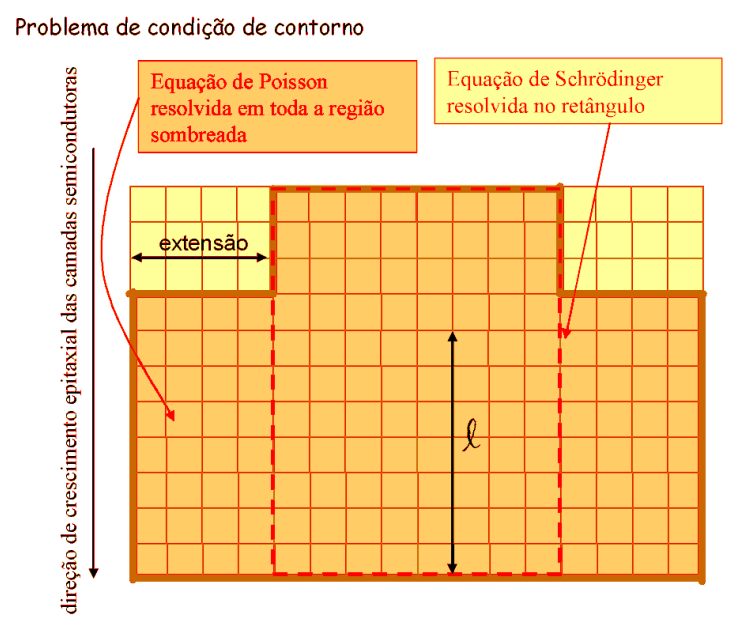

Figura 2.11: Representação esquemática da região de solução do cálculo auto-consistente das equações de Schrödinger e de Poisson, para estruturas como a da Figura (2.9-b). 
na Fig.(2.12). Trata-se de um trabalho de modelagem numérica de uma estrutura específica. Os resultados obtidos por Snider foram comparados com aqueles obtidos usando-se a formulação desenvolvida neste trabalho, para o caso bidimensional, comprovando sua validade. A estrutura analisada apresenta uma camada não-intencionalmente dopada de $A l_{1-x} G a_{x} A s$ crescida sobre um substrato semi-isolante, com uma dopagem de aceitadores $N_{a} \simeq 10^{14} \mathrm{~cm}^{-3}$, seguida por uma camada de poço quântico de GaAs com largura de $150 \AA$, uma camada espaçadora de $A l_{1-x} G a_{x} A s$ não-dopada, com uma largura de $50 \AA$, e sobre esta camada uma outra de $A l_{1-x} G a_{x} A s$ não-dopada com $N_{d}=10^{18} \mathrm{~cm}^{-3}$ e largura de $200 \AA$, e finalmente, uma última camada de GaAs não-dopado com largura de $150 \AA$ e $N_{d}=10^{18} \mathrm{~cm}^{-3}$.

\begin{tabular}{|c|c|c|c|}
\hline \multirow{2}{*}{$\begin{array}{r}150 \AA \\
1\end{array}$} & \multicolumn{2}{|c|}{$\longleftarrow$ Comprimento da "mesa" $\rightarrow$} & \multirow{2}{*}{$\underset{x}{\longrightarrow}$} \\
\hline & GaAs & $N_{0}=1 \times 10^{1818} \mathrm{~cm}^{3}$ & \\
\hline $200 \AA$ & AlGaAs & $x=0,3$ & $N_{0}=1 \times 10^{13} \mathrm{~cm}^{3}$ \\
\hline $50 \AA ̊$ & AlGaAs & $x=0,3$ & Intrinseco \\
\hline $150 \AA$ & GaAs & & Intrinseco \\
\hline & AlGaAs & $x=0,3$ & Intrinseco \\
\hline
\end{tabular}

Figura 2.12: Representação esquemática da estrutura de camadas semicondutoras representando uma mesa rasa, "shallow mesa", a qual é capaz de produzir um confinamento eletrostático lateral, devido ao "etching". A figura é retirada da Referência [13].

A solução numérica das Eqs.(2.47) e (2.49) constituem um problema de condição de contorno, como já apontado na Seção (2.2), em que a equação de Poisson é resolvida sobre a superfície inteira, ilustrada na Fig.(2.11), levando-se em conta a condição de contorno de superfície livre, como sendo a altura da barreira da superfície $V_{s}$ fixada pelo nível de Fermi ("Fermi-level pinning").

Os valores usados para o potencial de superfície no $G a A s$ é $V_{s}=0,6 \mathrm{eV}$ e para o $A l G a A s$ é $V_{s}=0,7 \mathrm{eV}$. Esses valores são escolhidos baseando-se em experimentos com estruturas 2-DEG [13]. A equação de Schrödinger é resolvida na região limitada da estrutura a qual contém elétrons, a fim de reduzir o esforço computacional. As funções de onda devem ser zero nas regiões de fronteira.

A discretização não-uniforme permite uma maior discretização na região de interesse, ou seja, na região onde existe uma concentração significativa de elétrons (região de confinamento), 
é feito $\Delta x=1 \sim 2 \AA$ e $\Delta y=10 \sim 20 \AA$. Por outro lado, nas regiões desprovidas de elétrons $\Delta x$ e $\Delta y$ podem assumir valores bem maiores.

Snider et al [13] não utilizam o esquema de discretização não-uniforme, por isso o esforço computacional é muito superior ao proposto por este método, e também não utilizam o método de "split-operator", o que aumenta consideravelmente o tempo computacional.

Utilizando o método numérico discutido na Seção (2.2) foi obtido o resultado mostrado na Fig.(2.13) para o perfil da banda de condução $V(x, 0)$ e a distribuição de elétrons $n(x, 0)$, para uma largura da mesa de $1500 \AA$ e temperatura de $4 \mathrm{~K}$.
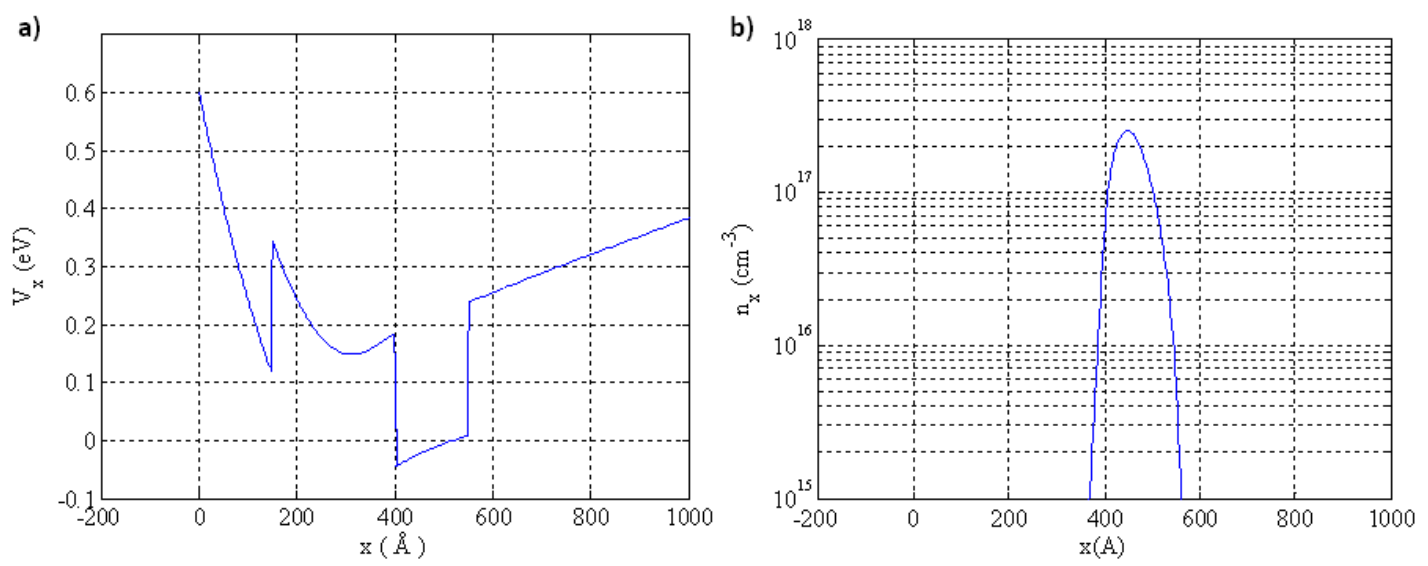

Figura 2.13: À esquerda, o perfil da banda de condução para o sistema apresentado na Fig.(2.12), e à direita a concentração de elétrons na região do fio quântico, ambos ao longo da direção x.

O resultado obtido na Fig.(2.13) pode ser comparado com aquele obtido por Snider et al [13], onde pode-se identificar a mesma posição para o pico da distribuição eletrônica na direção x, o qual se dá entre 50 e $60 \AA$ A, a partir da formação do poço quântico, confinados pelas barreiras das camadas de AlGaAs.

Estudando-se o sistema sem a formação da mesa, o que equivaleria a calcular o sistema unidimensional somente, estudado largamente na Ref. [50], nota-se que, mais de $200 \mathrm{meV}$ de confinamento, obtido no perfil de potencial da Fig.(2.13) provém da estrutura de mesa proposta na Fig.(2.12). Pode-se comparar também a banda de condução lateral, ao longo da direção y.

Como pode ser visto nas Figs.(2.13) e (2.14), os resultados obtidos através do método descrito neste trabalho estão em pleno acordo com aqueles obtidos na Ref. [13].

Para um aprofundamento da discussão de validação da ferramenta computacional apresentada na Seção (2.2), foi analisada outra estrutura, proposta por Laux et al, na Ref. [72], a 

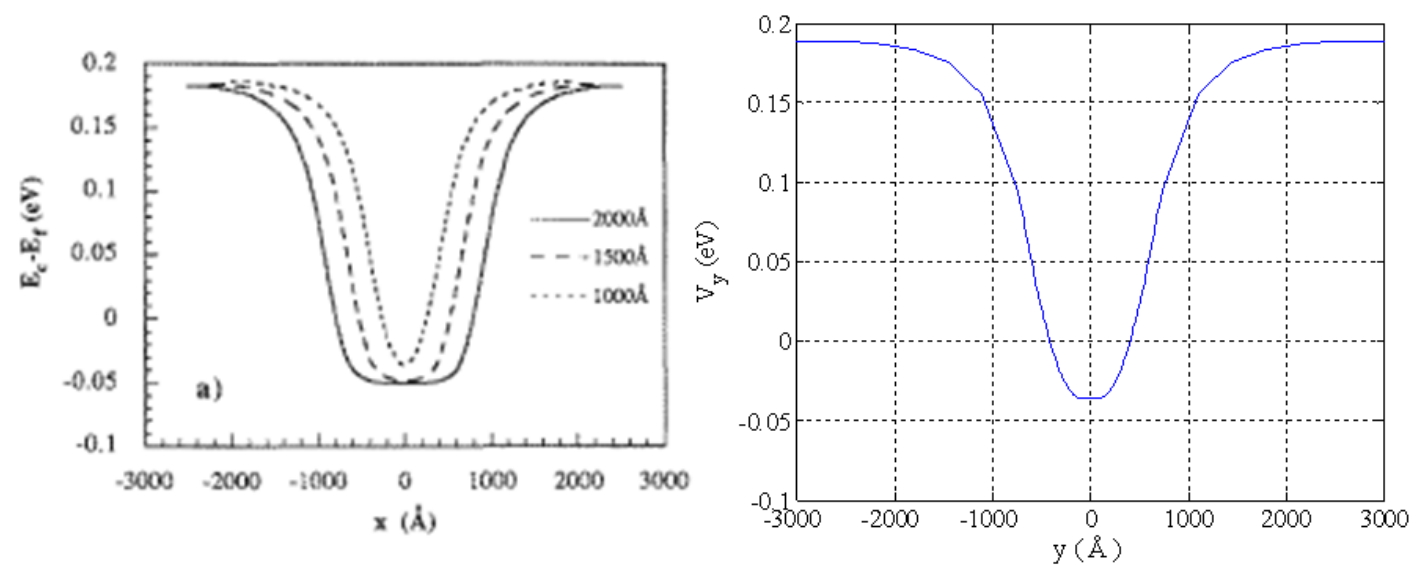

Figura 2.14: À esquerda pode-se observar o perfil de potencial ao longo da direção y, indicada por Snider pela letra x, na Ref. [13] para uma distância de 1500 Å. À direita, está mostrado o resultado obtido a partir da formulação apresentada na Seção (2.2).

qual é mostrada na Fig.(2.15).

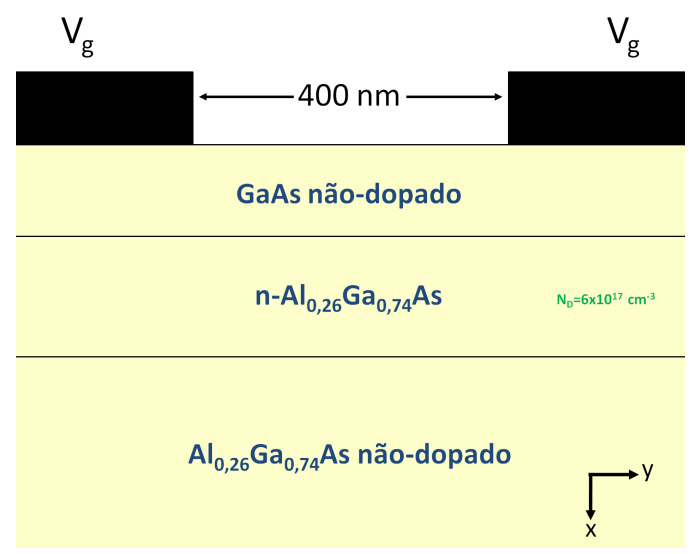

Figura 2.15: Geometria do dispositivo proposto na Ref. [72].

A estrutura considerada agora apresenta uma estrutura do tipo "split-gate" sobre uma heteroestrutura convencional de GaAs na Fig.(2.16), e foi extraída da Ref. [72].

Primeiramente, foram obtidos os resultados para o perfil de potencial lateral da estrutura mostrada na Fig.(2.15), para a temperatura de 4,2 K, e ao longo da direção y, próximo à interface $A l G a A s / G a A s$, no ponto $x=5,6 n m$.

Analisando os resultados obtidos através destas simulações pode-se concluir que é possível, para uma tensão negativa adequada no eletrodo de contato extinguir completamente a den- 

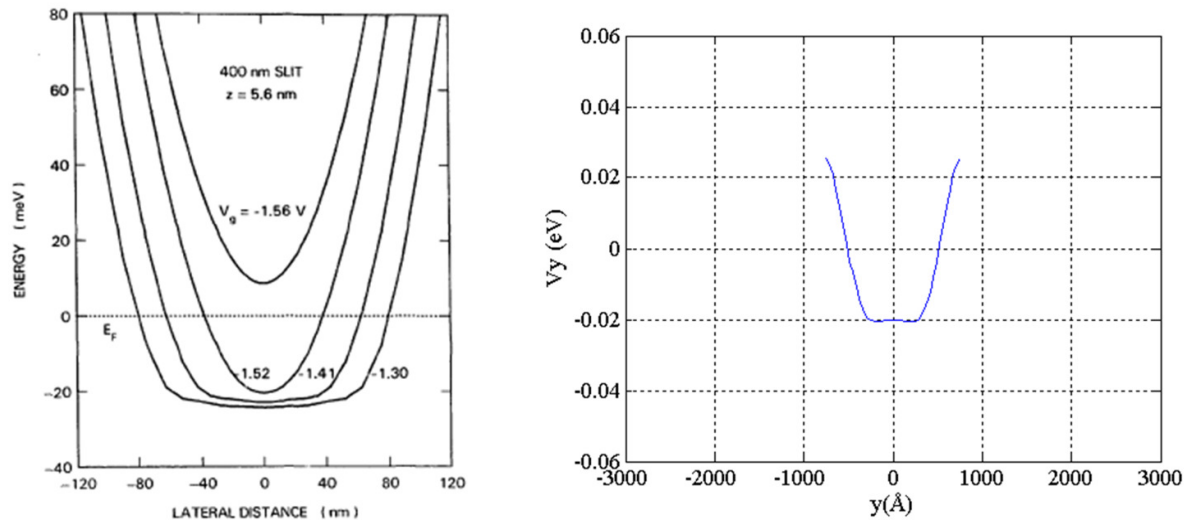

Figura 2.16: À esquerda, perfil de potencial na temperatura de 4,2 K, próximo à interface de AlGaAs obtida na Ref. [72]. À direita, os resultados obtidos a partir das simulações do método proposto na Seção $(2.2)$.

sidade eletrônica abaixo do eletrodo, e que, o canal formado sob a região sem "gate" (chamada "gate opening") também pode se depletar de elétrons, desde que o "gate opening" seja menor do que $0,5 \mu \mathrm{m}$, para esta estrutura. Os resultados mostrados na Ref. [72] são para um "gate opening" de $4 \mu \mathrm{m}$.

Observa-se que o potencial é aproximadamente parabólico quando há pouca ou nenhuma carga no canal, e que à medida que acumula cargas no poço, o perfil de potencial apresenta um perfil parabólico com um fundo chato. A densidade de elétrons também foi verificada para os mesmos parâmetros usados na obtenção da Fig.(2.17), para seis valores diferentes de tensão de "gate".

Uma forma característica ou um comportamento oscilatório surge na densidade de elétrons quando sucessivas sub-bandas contribuem para a densidade de carga do canal, confirmando, desta maneira a natureza 1D da estrutura. Estas oscilações talvez estejam associadas com as fortes oscilações encontradas em sistemas eletrônicos quasi-unidimensionais estudados por diversos autores [73]-[74].

De fato, o interesse nesse tópico foi renovado recentemente devido ao grande número de trabalhos teóricos e experimentais sobre oscilações observadas na condutância e transcondutância de sistemas eletrônicos quasi-unidimensionais, representando um consenso que as oscilações confirmam a natureza unidimensional do sistema.

De acordo com Crook et al [73] estas oscilações estão relacionadas com a densidade de probabilidade unidimensional 1D, a qual ele observou usando microscopia de varredura. 

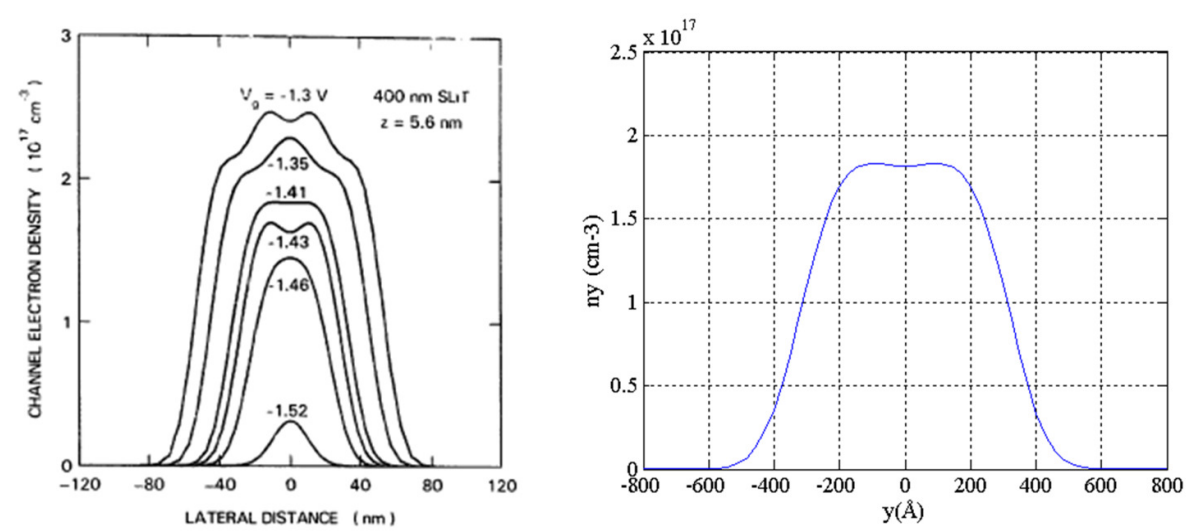

Figura 2.17: À esquerda é mostrado o resultado obtido por Laux et al [72] para a densidade eletrônica no canal unidimensional; à direita é mostrado um resultado obtido a partir da formulação proposta neste trabalho, devendo ser salientado que muitos parâmetros não são totalmente conhecidos para confrontar diretamente os resultados. Ainda assim, pode-se dizer que as simulações obtidas são satisfatórias.

Também, Pleutin et al [74] estudando também um sistema unidimensional, porém um fio de moléculas conectadas por pontes metálicas, observou oscilações ocorrendo no sistema eletrônico do fio.

Como foi mostrado na Seção (2.2) o formalismo apresentado permite estudar diversas geometrias diferentes, bastando, portanto, adaptar os parâmetros adequadamente para se obter os resultados desejados. Nesta seção, procura-se mostrar a possibilidade de se usar esta ferramenta computacional para investigação de transistores baseados em fios quânticos, simulando o comportamento da concentração de elétrons no canal condutor em função da tensão aplicada aos eletrodos, $V_{c}$.

A estrutura de camadas do dispositivo proposto é a mesma usada por Laux et al [72], e é mostrado na Fig.(2.18).

Aplicando-se uma tensão $V_{c}$ nos eletrodos de "gate" do dispositivo da Fig.(2.18) obtémse as curvas da Fig.(2.19) para o perfil de potencial lateral $V_{y}$, e a concentração eletrônica $n_{y}$.

Com os resultados obtidos através da formulação proposta pode-se encontrar uma curva para a concentração eletrônica por unidade de comprimento do fio $\left(n_{s}\right)$, mostrado na Fig.(2.20), à esquerda, e também a concentração eletrônica de pico em função de tensão aplicada aos eletrodos, mostrada à direita.

Utilizando estas curvas podem-se conhecer as características elétricas do dispositivo. Pode-se também, propor outros "designs" para se estudar o comportamento elétrico, estruturas 


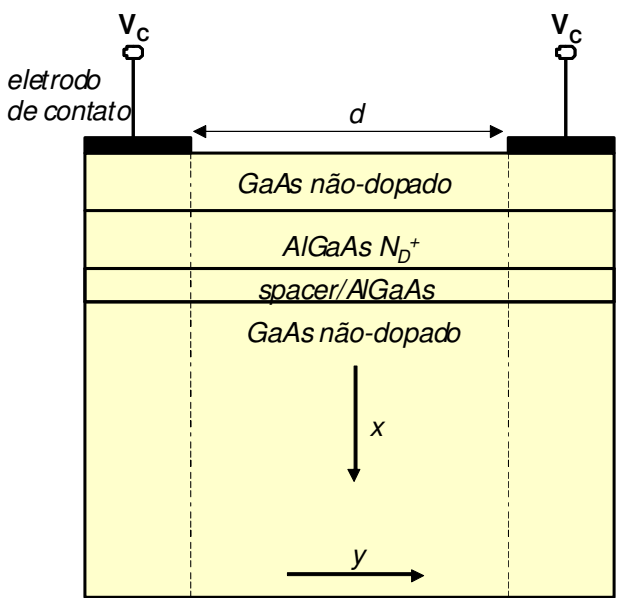

Figura 2.18: Geometria do dispositivo usado para se obter a relação entre a tensão Vc aplicada aos eletrodos de contato e a concentração eletrônica na interface AlGaAs/GaAs.

como o mostrado na Fig.(2.21), constituindo um transistor de fio quântico, que é o objetivo principal deste trabalho.

Neste capítulo, desenvolvemos o foco principal deste trabalho, que é o estudo numérico de heteroestruturas semicondutoras bidimensionais que apresentam um gás de eletrônico unidimensional (1DEG) formado a partir do confinamento eletrostático lateral de um gás eletrônico bidimensional (2DEG). A comparação dos resultados obtidos estão em pleno acordo com as disponíveis na literatura, fornecendo um concordância adequada, onde o formalismo proposto neste capítulo é uma ferramenta computacional bastante útil para investigar tanto estruturas unidimensionais quanto bidimensionais, compostas por dispositivos que empregam fios quânticos.

Tendo realizado todo o estudo numérico concernente a dispositivos de fios quânticos tais como, proposto por Snider et al, Ref. [13] Scherer et al, Ref. [14] indicados nas Figs.(1.2), (1.3), dentro da abordagem "top-down", deslocamos parte do nosso interesse para compreender e modelar também estruturas de fios quânticos do tipo mostrado na Fig.(1.10), usualmente fabricadas a partir das técnicas pertencentes a abordagem "bottom-up", que incorporam estruturas de tunelamento ressonante. Por essa razão, o próximo capítulo dedica-se a encontrar uma generalização para o cálculo de tunelamento ressonante supondo um estrutura contendo múltiplas barreiras de potencial. 

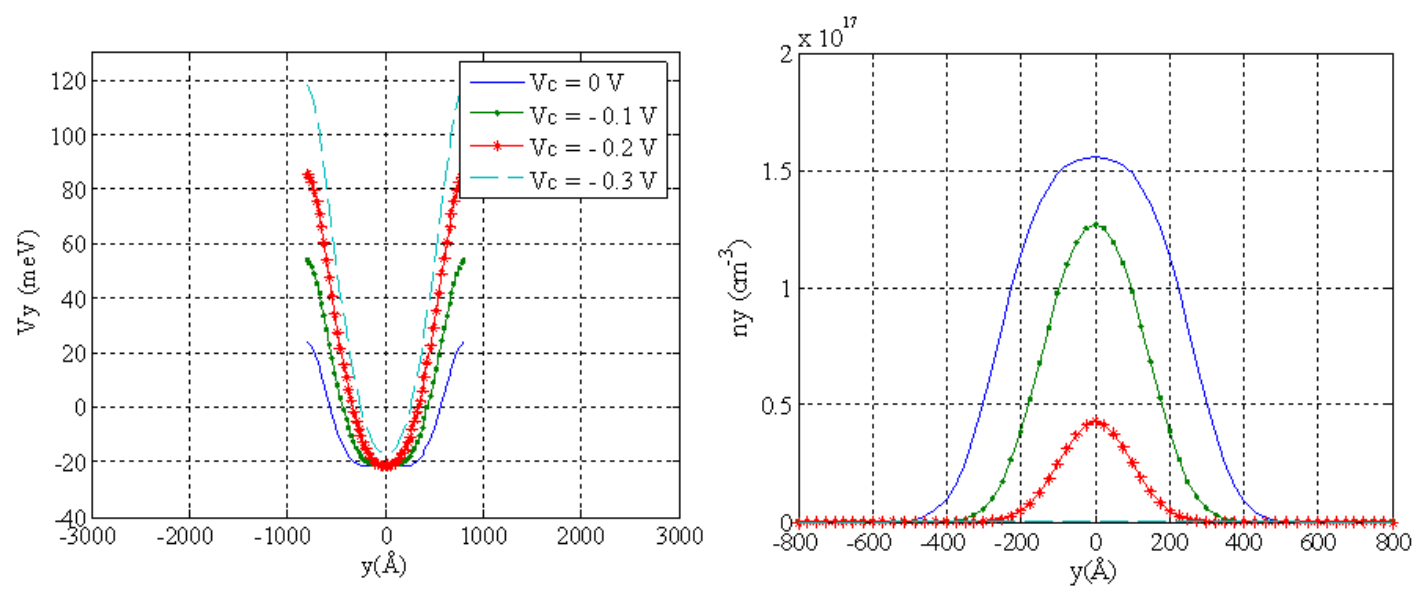

Figura 2.19: Perfil de potencial lateral, à esquerda, e concentração eletrônica na interface AlGaAs/GaAs em função da tensão $V_{C}$ aplicada aos eletrodos do dispositivo.
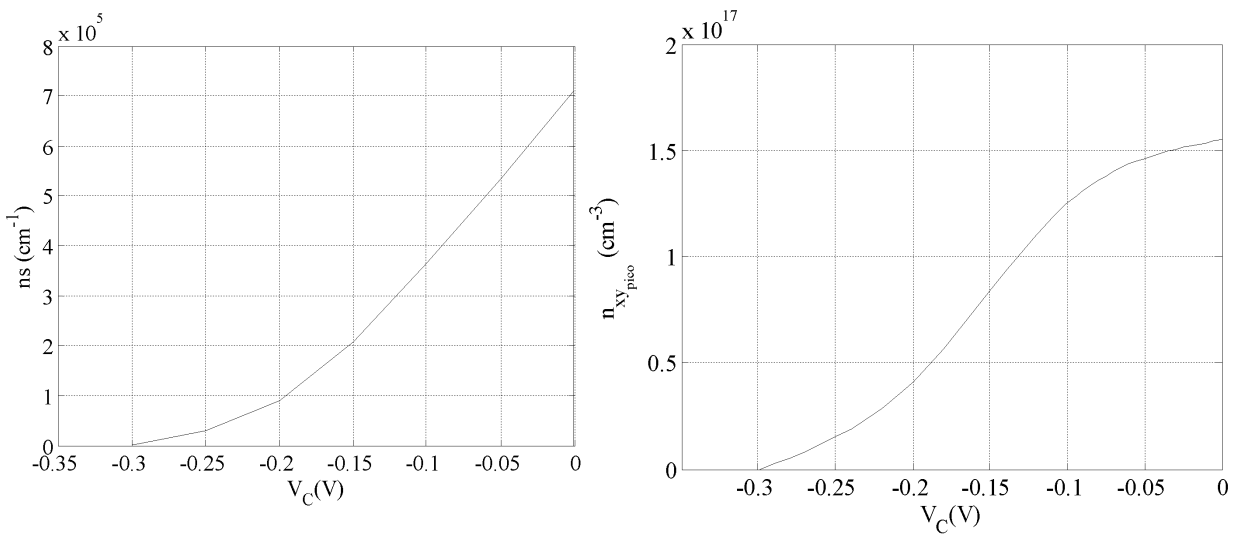

Figura 2.20: À esquerda é mostrado a densidade eletrônica por unidade de comprimento do fio $\left(\mathrm{n}_{s}\right)$ em função da tensão $V_{c}$ aplicada, e à direita, tem-se a tensão de pico alcançada para cada $V c$. 

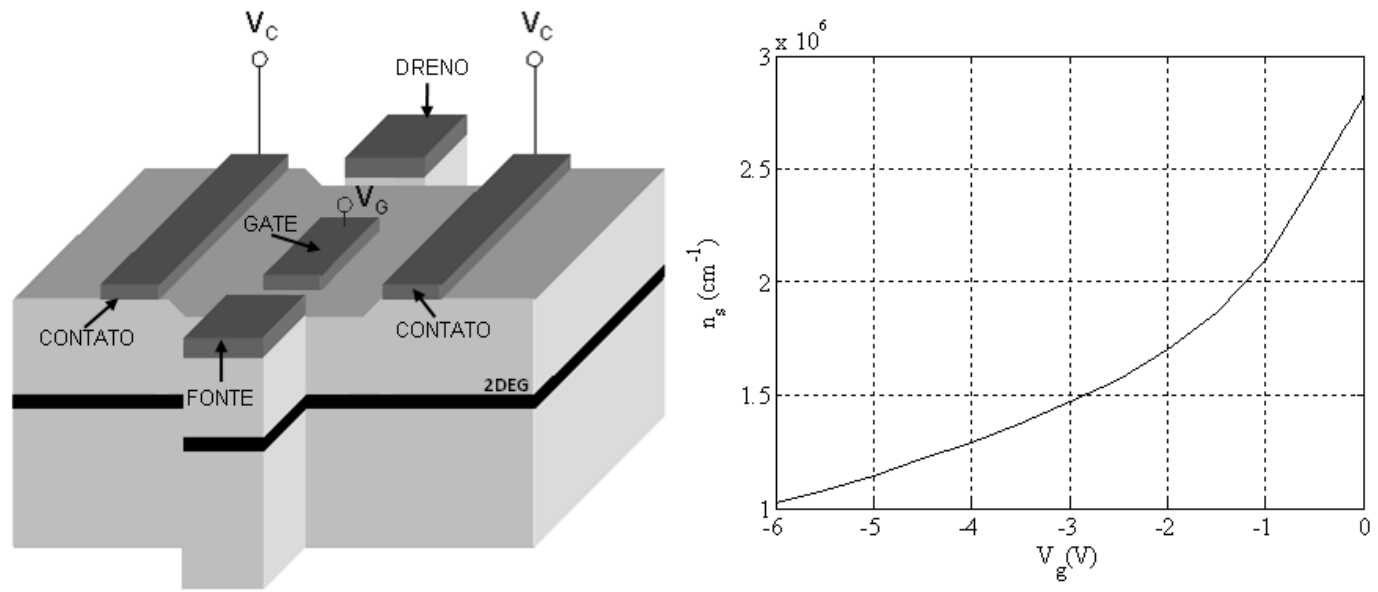

Figura 2.21: a) Um diagrama esquemático de um transistor de fio quântico; b) resultado para a densidade eletrônica controlada por uma tensão de gate, $V_{g}$ para um $V_{c}$ fixo. 


\section{Capítulo 3}

\section{Análise do Transporte Eletrônico pelo Formalismo da Matriz Transferência}

Neste capitulo é apresentado inicialmente um estudo analítico de transporte de corrente em heteroestruturas semicondutoras tipo super-rede [53], contendo uma sequência arbitrária de barreiras e poços quânticos. Estas heteroestruturas semicondutoras apresentam o fenômeno de tunelamento ressonante, amplamente aplicados em dispositivo de semicondutores volumétricos ("bulk"), são aqui estendidos para modelar diodos de fio quântico de tunelamento ressonante. O método empregado neste capítulo é o usual método da matriz transferência (MMT), o qual será convenientemente adaptado para poder ser utilizado em estruturas que apresentam simetria cilíndrica, tais como os fios quânticos mostrados na Fig.(1.10) [31].

\subsection{Fórmula de densidade de corrente de Tsu e Esaki}

Os sólidos em geral, são baseados em arranjos e estruturas ordenadas de um mesmo material com simetria translacional, e a teoria que os descreve, a teoria de bandas, assume que estas estruturas tem periodicidade infinita.

Por outro lado, as super-redes semicondutoras consistem em uma seqüência de camadas de diferentes materiais semicondutores, por exemplo, GaAs e $\mathrm{Ga}_{1-x} \mathrm{Al}_{x} \mathrm{As}$, arranjadas periodicamente, formando uma super-heteroestrutura infinita do tipo GaAs/ $\mathrm{Ga}_{1-x} \mathrm{Al}_{x} \mathrm{As} .$. GaAs/Ga $/{ }_{1-x} \mathrm{Al}_{x} \mathrm{As} \ldots \mathrm{GaAs} / \mathrm{Ga}_{1-x} \mathrm{Al}_{x} \mathrm{As}$. 
Então, para se produzir uma super-rede é necessário repetir uma seqüência de barreiras e poços quânticos acoplados, através da sobreposição adequada de camadas de materiais semicondutores convenientemente escolhidos, a fim de se obter as propriedades desejadas.

A partir de tal construção, ocorre uma quebra de simetria na periodicidade de um material sólido, como descrito pela teoria de bandas, introduzindo também, uma quebra na coerência das funções de onda que descreve o cristal, isto é, nas funções de Bloch. Consequentemente, haverá uma interpenetração das funções de onda das barreiras com a dos poços quânticos. Essas interações entre as funções de onda podem ser descritas a partir da mecânica quântica, e dão origem à mini-bandas de energia, tanto para os elétrons como para as lacunas, ao longo da super-rede, afetando os fenômenos de transporte de cargas através de toda a estrutura.

Note que é usual denominar super-rede uma seqüência infinita de poços quânticos acoplados de largura $L$, separados por barreiras de espessuras $V_{0}$. Em nosso trabalho, preferimos não usar o termo super-rede, e sim super-heteroestrutura, uma vez, que os dispositivos propostos empregam um segmento de uma super-rede, posto que, interfaces entre as superredes e os eletrodos são inevitáveis.

A fabricação de tais super-heteroestruturas é obtida a partir de técnicas de crescimento epitaxial, que são muito relevantes na área de dispositivos eletrônicos e optoeletrônicos. Devido as dimensões das estruturas de interesse ser da ordem de nanômetros, o tratamento quântico de tais sistemas se torna relevante e necessário para compreensão do comportamento do sistema, pois manifestações quânticas, tais como tunelamento e confinamento eletrônico, são importantes mecanismos que afetam as propriedades ópticas e de transporte do dispositivo.

Dada a importância do estudo do transporte eletrônico em dispositivos semicondutores de super-heteroestruturas, neste capítulo calculamos inicialmente a densidade de corrente $J$ geral, para uma seqüências de barreiras e poços quânticos, como proposto originalmente por Esaki e Tsu na Ref. [53], a fim de se construir uma ferramenta de cálculo para compreensão do comportamento da característica $\mathbf{I}-\mathbf{V}$ de tais estruturas. Propriedades de transporte significativas foram encontradas nesses sistemas, tais como, condutividade diferencial negativa e oscilações de Bloch [53].

O perfil de potencial de uma super-heteroestrutrura de $G a A s / G a_{1-x} A l_{x} A s$ é ilustrado esquematicamente na Fig.(3.1-a), para o caso de uma super-heteroestrutrura em estado de equilíbrio, sem polarização aplicada. A Fig.(3.1-b), por outro lado, mostra a mesma superheteroestrutrura, no entanto, com uma polarização $V$ aplicada. Observe que, a aplicação de 
uma tensão $V$ entre os terminais dessa super-heteroestrutura, inclina o perfil de potencial, deslocando-o em relação ao nível de Fermi da situação em equilíbrio.

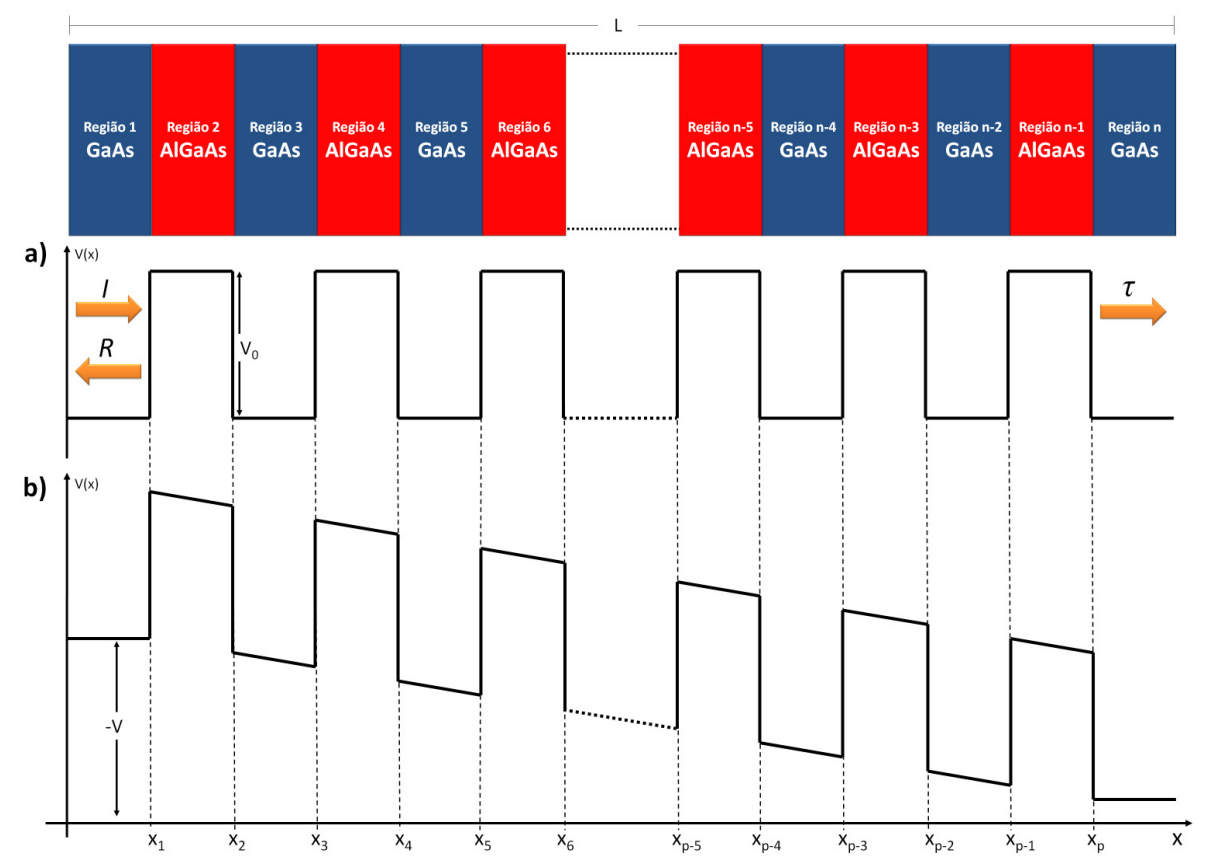

Figura 3.1: a) Perfil de potencial sem a aplicação de polarização. b) Perfil de potencial com uma tensão aplicada $V$.

Estruturas como as mostradas nas Figs.(3.1-a) e (3.1-b) são investigadas usualmente na literatura [53] assumindo-se o modelo do elétron livre mas seguindo algumas restrições. Especificamente, assume-se o modelo do elétron livre, no qual o potencial da rede cristalina é ignorado e os elétrons são considerados livres para se moverem ao longo da rede, com funções de onda do tipo Bloch, e um potencial periódico $V(x)$, como mostrado na Fig.(3.1). Todavia, para tornar o modelo mais realístico, ao invés de considerar a massa do elétron livre, $m_{0}$, costuma-se assumir uma massa efetiva $m^{*}$, para o elétron num determinado material, baseado na aproximação parabólica da energia do elétron $E$ em função do vetor de onda $\mathbf{k}$, segundo a seguinte relação [48]:

$$
\frac{1}{m^{*}}=\left(\frac{1}{\hbar^{2}}\right)\left(\frac{d^{2} E(k)}{d k^{2}}\right)
$$

Assim sendo, em cada camada da estrutura, o elétron possui uma massa efetiva $m^{*}$ diferente, correspondente à camada semicondutora em que se encontra. A equação de Schrödinger, para o sistema unidimensional em consideração, ao longo da direção $x$, a qual representa a direção ao longo das múltiplas barreiras de potencial, pode ser escrita na forma 


$$
-\frac{\hbar^{2}}{2 m} \frac{d^{2}}{d x^{2}} \Psi_{n}+V(x) \Psi_{n}=E \Psi_{n}
$$

onde $E$ é a energia total do elétron, que pode ser representada pela soma das energias transversal e longitudinal do portador de carga.

Portanto, para uma super-heteroestrutura como a mostrada na Fig.(3.1-a), as funções de onda dos elétrons [48], à esquerda, a qual chamaremos de região 1, e à direita, a qual chamaremos de região $\mathbf{n}$, correspondentes às regiões dos contatos, podem ser escritas como

$$
\begin{gathered}
\Psi_{1}=\exp \left(i k_{1} x\right)+R \exp \left(-i k_{1} x\right) \\
\Psi_{n}=\tau \exp \left(i k_{n} x\right)
\end{gathered}
$$

em que $R$ e $\boldsymbol{\tau}$ são as amplitudes de reflexão e transmissão da função de onda que caracteriza o elétron livre.

Vale a pena mencionar que, para que haja fluxo de corrente através da estrutura e consequentemente transporte de carga, o sistema deve ser perturbado por uma polarização externa $V$, deslocando o perfil de potencial $V(x)$ como mostrado na Fig.(3.1-b). Assim, a densidade de corrente total, $J_{\text {total }}$, da estrutura mostrada na Fig.(3.1-b), pode ser obtida através da diferença entre densidades de corrente da região $\mathbf{1}, J_{1}$, e da região $\mathbf{n}, J_{n}$, o que pode ser obtido através da relação

$$
J_{\text {total }}=J_{1}-J_{n}
$$

sendo $J_{1}$ o fluxo em uma direção apenas, elétrons vindo da região $\mathbf{1}$ para a região $\mathbf{n}$ e $J_{n}$ o fluxo de elétrons vindo da região $\mathbf{n}$ para a região $\mathbf{1}$. Nota-se que, na situação de equilíbrio, não há fluxo de cargas, isto é, $J_{\text {total }}=0$.

A partir da teoria de tunelamento em sólidos de Duke [75], a densidade de corrente, $J_{1}$, é dada por

$$
J_{1}=\frac{\int \mathbf{T} \hbar k A^{2} \rho(k) d k}{m^{*}}
$$

onde, $\mathbf{T}$ é a probabilidade de tunelamento da carga através da super-heteroestrutura, $A$ é uma constante de normalização, $\rho(\mathbf{k})$ é a densidade de ondas incidentes, $\mathbf{k}$ é o vetor de onda, e ћ é a constante de Planck dividido por $2 \pi$. 
Adicionalmente, para encontrarmos a densidade de corrente de tunelamento $J_{1}$ nas super-heteroestruturas de interesse precisamos introduzir a energia $E$, a qual representa a energia do elétron incidente, proveniente da região 1 , e $E^{\prime}$, a energia do elétron transmitido, para a região n. Além disso, como estamos estudando sistemas constituídos por semicondutores a função distribuição de Fermi-Dirac, $f_{1}(E)$, deve ser levada em consideração na Eq.(3.5), bem como o número de estados acessíveis do sistema, $\left(1-f_{n}(E)\right)$.

Através de todas as considerações acima, a densidade de corrente de transporte $J_{1}$ será dada por, Ref. [75]

$$
J_{1}=\left(\frac{2 s+1}{8 \pi^{3} \hbar}\right) q \int d k_{l} d^{2} k_{t} f_{1}(E)\left(1-f_{n}(E)\right) \mathbf{T}\left(E_{l}\right)\left(\frac{\partial E_{l}}{\partial k_{l}}\right)
$$

onde $s$ é o spin, $q$ é a carga elementar do elétron, e $\mathbf{T}\left(E_{l}\right)$ é a probabilidade de tunelamento em função da energia longitudinal. A energia total, $E$, pode ser obtida através da soma das energias longitudinal (direção $x$ ), $E_{l}$, e transversal (direção $y$ ), $E_{t}$.

$$
E=E_{l}+E_{t}
$$

sendo que a energia transversal é dada por

$$
E_{t}=\frac{\hbar^{2} k_{t}^{2}}{2 m^{*}}
$$

Tomando-se a derivada da Eq.(3.8) em relação ao vetor de onda na direção transversal, $k_{t}$, obtêm-se

$$
d^{2} k_{t}=\frac{2 m^{*}}{\hbar^{2}} d E_{t}
$$

Por outro lado, diferenciando-se a Eq.(3.7) em função do vetor de onda na direção longitudinal, $k_{l}$, obtêm-se

$$
\frac{\partial E}{\partial k_{l}}=\frac{\partial E_{l}}{\partial k_{l}}
$$

A partir dos resultados encontrados nas Eqs.(3.9) e (3.10) e substituindo na Eq.(3.6) pode-se escrever

$$
J_{1}=\left(\frac{2 s+1}{8 \pi^{3} \hbar}\right) q \int d k_{l}\left(\frac{2 m^{*}}{\hbar^{2}} d E_{t}\right) f_{1}(E)\left(1-f_{n}(E)\right) \mathbf{T}\left(E_{l}\right)\left(\frac{\partial E_{l}}{\partial k_{l}}\right)
$$


rearranjando os termos:

$$
\begin{gathered}
J_{1}=\left(\frac{2 s+1}{8 \pi^{3} \hbar}\right)\left(\frac{2 m^{*} q}{\hbar^{2}}\right) \int\left(d k_{l}\right)\left(d E_{t}\right) f_{1}(E)\left(1-f_{n}(E)\right) \mathbf{T}\left(E_{l}\right)\left(\frac{\partial E_{l}}{\partial k_{l}}\right) \\
J_{1}=\left(\frac{2 s+1}{8 \pi^{3} \hbar}\right)\left(\frac{2 m^{*} q}{\hbar^{2}}\right) \int f_{1}(E)\left(1-f_{n}(E)\right) d E_{t} \mathbf{T}\left(E_{l}\right) d E_{l}
\end{gathered}
$$

Como o sistema considerado trata essencialmente de elétrons, que são férmions, pode-se imediatamente considerar $s=1 / 2$ na Eq.(3.12), de modo que a densidade de corrente $J_{1}$ pode ser reduzida a

$$
J_{1}=\left(\frac{1}{4 \pi^{3}} \frac{m^{*} q}{\hbar^{3}}\right) \int_{0}^{\infty} \int_{0}^{\infty} f_{1}(E)\left(1-f_{n}(E)\right) d E_{t} \mathbf{T}\left(E_{l}\right) d E_{l}
$$

Analogamente, a densidade de corrente $J_{n}$, correspondendo ao fluxo de elétrons provenientes da região $\mathbf{n}$ pode ser escrita como

$$
J_{n}=\left(\frac{1}{4 \pi^{3}} \frac{m^{*} q}{\hbar^{3}}\right) \int_{0}^{\infty} \int_{0}^{\infty} f_{n}(E)\left(1-f_{1}(E)\right) d E_{t} \mathbf{T}\left(E_{l}\right) d E_{l}
$$

Sendo assim, substituindo as Eqs.(3.13) e (3.14) na Eq.(3.4), pode-se obter a corrente total

$$
J_{T}=\left(\frac{1}{4 \pi^{3}} \frac{m^{*} q}{\hbar^{3}}\right) \int_{0}^{\infty} \int_{0}^{\infty}\left[f_{1}(E)-f_{n}(E)\right] d E_{t} \mathbf{T}\left(E_{l}\right) d E_{l}
$$

sabendo que a função distribuição de Fermi-Dirac é

$$
f_{i}(E)=\frac{1}{1+\exp \left(\frac{E_{l}+E_{\mathrm{t}}-E_{F}}{k_{B} T}\right)},
$$

onde $k_{B}$ é a constante de Boltzmann.

Calculando a integral em $d E_{t}$ na Eq.(3.15) e realizando algumas manipulações algébricas, tem-se finalmente a densidade de corrente total, no qual iremos chamar apenas de $J$, obtida por Esaki e Tsu na Ref.[53] é

$$
J=\left[\frac{m^{*} q k_{B} T}{2 \pi^{3} \hbar^{3}}\right] \int_{0}^{\infty} \mathbf{T}\left(E_{l}\right)\left[\ln \left(\frac{1+\exp \left(\frac{E_{F}-E_{l}}{k_{B} T}\right)}{1+\exp \left(\frac{E_{F}-E_{l}-q V}{k_{B} T}\right)}\right)\right] d E_{l}
$$

A integral mostrada na Eq.(3.17) não apresenta uma solução analítica fechada, precisando portanto de um tratamento numérico para ser avaliada. 


\subsubsection{Densidade de corrente J no limite de baixas temperaturas}

Sendo assim, para realizar um estudo preliminar de uma dada estrutura, pode-se investigar inicialmente o comportamento da densidade de corrente de tunelamento expressa na Eq.(3.17) para temperatura baixas, como realizado por Tsu e Esaki na Ref. [53] , na qual toma-se o limite da temperatura tendendo para zero. Considerando esse limite, há algumas aproximações que tornam a integral mais facilmente tratável. Do ponto de vista do cálculo pode-se matematicamente dividir $J$ em dois casos:

O primeiro caso ocorre quando $q V>E_{F}$. Assim, assumindo-se a Eq.(3.17) no limite em que $T \rightarrow 0$ tem-se

$$
J=\lim _{T \rightarrow 0 K}\left\{\left[\frac{m^{*} q k_{B} T}{2 \pi^{3} \hbar^{3}}\right] \int_{0}^{\infty} \mathbf{T}\left(E_{l}\right)\left[\ln \left(\frac{1+\exp \left(\frac{E_{F}-E_{l}}{k_{B} T}\right)}{1+\exp \left(\frac{E_{F}-E_{l}-q V}{k_{B} T}\right)}\right)\right] d E_{l}\right\}
$$

Separando a Eq.(3.18) nos intervalos de integração tem-se

$$
\begin{aligned}
J= & {\left[\frac{m^{*} q K_{B}}{2 \pi^{3} \hbar^{3}}\right] \lim _{T \rightarrow 0 K}\left\{T \int_{0}^{E_{F}} \mathbf{T}\left(E_{l}\right)\left[\ln \left(\frac{1+\exp \left(\frac{E_{F}-E_{l}}{k_{B} T}\right)}{1+\exp \left(\frac{E_{F}-E_{l}-q V}{k_{B} T}\right)}\right)\right] d E_{l}\right\} } \\
& +\left[\frac{m^{*} q K_{B}}{2 \pi^{3} \hbar^{3}}\right] \lim _{T \rightarrow 0 K}\left\{T \int_{E_{F}}^{\infty} \mathbf{T}\left(E_{l}\right)\left[\ln \left(\frac{1+\exp \left(\frac{E_{F}-E_{l}}{k_{B} T}\right)}{1+\exp \left(\frac{E_{F}-E_{l}-q V}{k_{B} T}\right)}\right)\right]\left(d E_{l}\right)\right\}
\end{aligned}
$$

Como $q V>E_{F}$, temos as seguintes aproximações:

$$
\exp \left(\frac{E_{F}-E_{l}-q V}{k_{B} T}\right)<<1 \quad \text { e } \quad \exp \left(\frac{E_{F}-E_{l}}{k_{B} T}\right)>>1
$$

que substituídas na Eq.(3.19) fornecem

$$
\begin{aligned}
J= & {\left[\frac{m^{*} q k_{B}}{2 \pi^{3} \hbar^{3}}\right] \lim _{T \rightarrow 0 K}\left\{T \int_{0}^{E_{F}} \mathbf{T}\left(E_{l}\right)\left[\ln \left(\frac{\exp \left(\frac{E_{F}-E_{l}}{k_{B} T}\right)}{1}\right)\right] d E_{l}\right\}+} \\
& +\left[\frac{m^{*} q k_{B}}{2 \pi^{3} \hbar^{3}}\right] \lim _{T \rightarrow 0 K}\left\{T \int_{E_{F}}^{\infty} \mathbf{T}\left(E_{l}\right) \ln (1) d E_{l}\right\} \\
= & {\left[\frac{m^{*} q k_{B}}{2 \pi^{3} \hbar^{3}}\right] \lim _{T \rightarrow 0 K}\left\{T \int_{0}^{E_{F}} \mathbf{T}\left(E_{l}\right)\left[\ln \left(\exp \left(\frac{E_{F}-E_{l}}{K_{B} T}\right)\right)\right] d E_{l}\right\} }
\end{aligned}
$$

Manipulando a Eq.(3.20) tem-se 


$$
J=\left[\frac{m^{*} q k_{B}}{2 \pi^{3} \hbar^{3}}\right] \lim _{T \rightarrow 0 K}\left\{T \int_{0}^{E_{F}} \mathbf{T}\left(E_{l}\right)\left(\frac{E_{F}-E_{l}}{k_{B} T}\right) d E_{l}\right\}
$$

Por fim

$$
J=\left[\frac{m^{*} q}{2 \pi^{3} \hbar^{3}}\right] \lim _{T \rightarrow 0 K}\left\{k_{B} T \int_{0}^{E_{F}} \mathbf{T}\left(E_{l}\right)\left(\frac{E_{F}-E_{l}}{k_{B} T}\right)\left(d E_{l}\right)\right\}
$$

Finalmente, a densidade de corrente na aproximação de baixas temperaturas para $q V>E_{F}$

$$
J=\left[\frac{m^{*} q}{2 \pi^{3} \hbar^{3}}\right] \int_{0}^{E_{F}} \mathbf{T}\left(E_{l}\right)\left(E_{F}-E_{l}\right) d E_{l}
$$

No segundo caso considerado, toma-se novamente a Eq.(3.17) e assume-se $q V<E_{F}$, na aproximação de baixas temperaturas, assim

$$
\begin{aligned}
J= & {\left[\frac{m^{*} q k_{B}}{2 \pi^{3} \hbar^{3}}\right] \lim _{T \rightarrow 0 K}\left\{T \int_{0}^{E_{F}-q V} \mathbf{T}\left(E_{l}\right)\left[\ln \left(\frac{1+\exp \left(\frac{E_{F}-E_{l}}{k_{B} T}\right)}{1+\exp \left(\frac{E_{F}-E_{l}-q V}{k_{B} T}\right)}\right)\right] d E_{l}\right\}+} \\
& {\left[\frac{m^{*} q k_{B}}{2 \pi^{3} \hbar^{3}}\right] \lim _{T \rightarrow 0 K}\left\{T \int_{E_{F}-q V}^{E_{F}} \mathbf{T}\left(E_{l}\right)\left[\ln \left(\frac{1+\exp \left(\frac{E_{f}-E_{l}}{k_{B} T}\right)}{1+\exp \left(\frac{E_{f}-E_{l}-q V}{k_{B} T}\right)}\right)\right] d E_{l}\right\} }
\end{aligned}
$$

Tomando-se $q V-E_{F}<0$, isto é, $E_{F}-q V>0$. De outro lado, considerando $0<E_{l}<$ $E_{F}-q V$, tem-se

$$
\exp \left(\frac{E_{F}-E_{l}-q V}{K_{B} T}\right)>>1 \quad \text { e } \quad \exp \left(\frac{E_{F}-E_{l}}{K_{B} T}\right)>>1
$$

e $E_{F}-q V<E_{l}<E_{F}$, tem-se

$$
\exp \left(\frac{E_{F}-E_{l}-q V}{K_{B} T}\right)<<1 \quad \text { e } \quad \exp \left(\frac{E_{F}-E_{l}}{K_{B} T}\right)>>1
$$

portanto,

$$
\begin{aligned}
J= & {\left[\frac{m^{*} q k_{B}}{2 \pi^{3} \hbar^{3}} \lim _{T \rightarrow 0 K}\left\{T \int_{0}^{E_{F}-q V} \mathbf{T}\left(E_{l}\right)\left[\ln \left(\frac{\exp \left(\frac{E_{F}-E_{l}}{K_{B} T}\right)}{\exp \left(\frac{E_{F}-E_{l}-q V}{K_{B} T}\right)}\right)\right] d E_{l}\right\}+\right.} \\
& +\left[\frac{m^{*} q k_{B}}{2 \pi^{3} \hbar^{3}}\right] \lim _{T \rightarrow 0 K}\left\{T \int_{E_{F}-q V}^{E_{F}} \mathbf{T}\left(E_{l}\right)\left[\ln \left(\frac{\exp \left(\frac{E_{f}-E_{l}}{K_{B} T}\right)}{1}\right)\right] d E_{l}\right\}
\end{aligned}
$$


Rearranjando a Eq.(3.25)

$$
\begin{aligned}
J= & {\left[\frac{m^{*} q k_{B}}{2 \pi^{3} \hbar^{3}}\right] \lim _{T \rightarrow 0 K}\left\{T \int_{0}^{E_{F}-q V} \mathbf{T}\left(E_{l}\right)\left[\ln \left(\exp \left(\frac{q V}{k_{B} T}\right)\right)\right] d E_{l}\right\}+} \\
& +\left[\frac{m^{*} q k_{B}}{2 \pi^{3} \hbar^{3}}\right] \lim _{T \rightarrow 0 K}\left\{T \int_{E_{F}-q V}^{E_{F}} \mathbf{T}\left(E_{l}\right)\left[\ln \left(\exp \left(\frac{E_{F}-E_{l}}{k_{B} T}\right)\right)\right] d E_{l}\right\}
\end{aligned}
$$

Com isso, tem-se

$$
\begin{aligned}
J= & {\left[\frac{m^{*} q}{2 \pi^{3} \hbar^{3}}\right] \lim _{T \rightarrow 0 K}\left\{k_{B} T \int_{0}^{E_{F}-q V} \mathbf{T}\left(E_{l}\right)\left(\frac{q V}{k_{B} T}\right) d E_{l}\right\}+} \\
& +\left[\frac{m^{*} q}{2 \pi^{3} \hbar^{3}}\right] \lim _{T \rightarrow 0 K}\left\{k_{B} T \int_{E_{F}-q V}^{E_{F}} \mathbf{T}\left(E_{l}\right)\left(\frac{E_{F}-E_{l}}{k_{B} T}\right) d E_{l}\right\}
\end{aligned}
$$

Finalmente, assumindo-se $T \rightarrow 0$, obtém-se

$$
J=\left[\frac{m^{*} q}{2 \pi^{3} \hbar^{3}}\right]\left\{q V \int_{0}^{E_{F}-q V} \mathbf{T}\left(E_{l}\right) d E_{l}+\int_{E_{F}-q V}^{E_{F}} \mathbf{T}\left(E_{l}\right)\left(E_{F}-E_{l}\right) d E_{l}\right\}
$$

O formalismo desenvolvido neste capítulo é baseado no trabalho proposto por Esaki e Tsu, em 1973 [53], e fornece as expressões dadas pelas Eqs.(3.23) e (3.28) no limite de $T \rightarrow 0$. Por outro lado, a Eq.(3.17) fornece a densidade de corrente, $J$ para qualquer temperatura T, a qual exige o cálculo do coeficiente de transmissão, ou a probabilidade de tunelamento, $\mathbf{T}(\mathrm{E})$. Uma modelagem analítica para o cálculo do coeficiente de transmissão, T, dos elétrons para tais sistemas é desenvolvido detalhadamente nas seções seguintes deste capítulo, baseado no Método da Matriz Transferência (MMT) em espaço real. Note que a equação para a densidade de corrente de Tsu-Esaki pode ser adequada para sistemas de dimensionalidade reduzida, bastando para isso escolher convenientemente a densidade de estados pertinente aos sistemas 2D, 1D ou 0D. Conhecendo-se as técnicas descritas nas próximas seções podemos usá-las adequadamente de modo a fornecer uma melhor compreensão acerca das propriedades eletrônicas dos dispositivos de interesse. 


\subsection{Cálculo do coeficiente de transmissão pelo Método da Ma- triz Transferência para múltiplas barreiras de potencial com e sem polarização aplicada}

O foco do nosso trabalho, nesta seção, é o estudo de estruturas semicondutoras que apresentam barreiras de potencial, podendo ser representadas fisicamente por dispositivos que empregam várias camadas semicondutoras dispostas numa sequência arbitrária de barreiras e poços quânticos. Sistemas envolvendo múltiplos poços quânticos nada mais são do que uma extensão natural de casos muito simplificados envolvendo um único poço quântico ou uma única barreira de potencial. Esses casos simples são encontrados nos livros textos de cursos elementares, [76] e [77], e são apresentados no Apêndice B como uma revisão de conceitos fundamentais de mecânica quântica para solução de poços quânticos.

Nesta seção, são investigadas estruturas com e sem polarização, a fim de obter uma generalização para um número arbitrário de barreiras de potencial para ambos os casos (com e sem aplicação de um campo elétrico externo). A abordagem utilizada nesta seção é frequentemente conhecida na literatura como Método da Matriz Transferência (MMT) [48].

\subsubsection{Uma barreira de potencial com polarização externa aplicada}

Inicialmente iremos estudar o cálculo do coeficiente de transmissão, T, para heteroestruturas semicondutoras com um campo elétrico externo aplicado. A aplicação de um campo elétrico inclina o perfil de potencial retangular usado para descrever os casos em equilíbrio, isto é, na ausência de polarização. Uma aproximação usual para a deformação sofrida pela banda de condução no caso de uma única barreira pode ser considerada visualizando-se a Fig.(3.2), a qual mostra um potencial inclinado. O potencial externo modifica as soluções da equação de Schrodinger na região da barreira, que se tornam uma função da tensão $V$ aplicada.

\section{Solução analítica}

Nesta Seção é apresentada a solução analítica do cálculo do coeficiente de transmissão, $\mathbf{T}$, para uma heteroestrutura com uma única barreira de potencial, sujeita a uma diferença de potencial, $V$. A equação de Schrödinger será resolvida em cada região separadamente, sendo posteriomente impostas as condições de contorno apropriadas. 


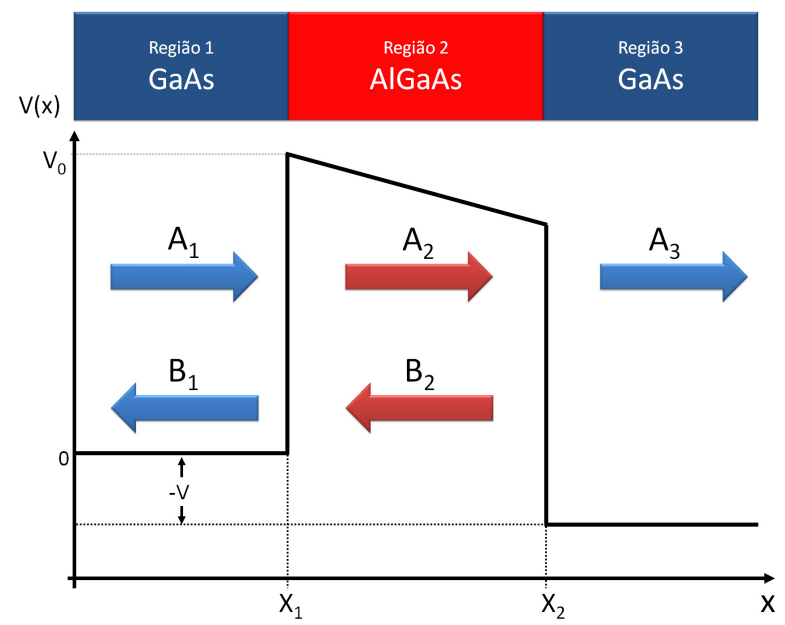

Figura 3.2: Perfil de potencial de uma barreira de potencial com polarização aplicada.

\section{Região 1:}

$$
-\frac{\hbar^{2}}{2 m_{1}^{*}} \frac{d^{2} \Psi_{1}(x)}{d x^{2}}=E \Psi_{1}(x) \rightarrow \frac{d^{2} \Psi_{1}(x)}{d x^{2}}+\frac{2 m_{1}^{*}}{\hbar^{2}} E \Psi_{1}(x)=0
$$

onde $k_{1}=\sqrt{\frac{2 m_{1}^{*}}{\hbar^{2}} E}$

$$
\frac{d^{2} \Psi_{1}(x)}{d x^{2}}+k_{1}^{2} \Psi_{1}(x)=0
$$

Como solução da Eq.(3.29)

$$
\Psi_{1}(x)=C_{1} \exp \left(i k_{1} x\right)+D_{1} \exp \left(-i k_{1} x\right)
$$

\section{Região 2:}

Como pode ser visto na Fig.(3.2), esta região é representada por um potencial inclinado, que de acordo com a geometria do diagrama pode ser facilmente obtida escrevendo-se

$$
V(x)=V_{n}+s_{n} x
$$

no qual

$$
s_{n}=-\frac{q V}{\left(x_{2}-x_{1}\right)}
$$

corresponde à inclinação do potencial. Sendo assim, a equação de Schrödinger pode ser reescrita como

$$
\frac{\hbar^{2}}{2 m^{*}} \frac{d^{2} \Psi_{n}(x)}{d x^{2}}-\left(V_{n}+s_{n} x\right) \Psi_{n}(x)+E \Psi_{n}(x)=0 \rightarrow
$$




$$
\rightarrow \frac{d^{2} \Psi_{n}(x)}{d x^{2}}-\frac{2 m^{*}}{\hbar^{2}}\left[\left(V_{n}+s_{n} x\right)-E\right] \Psi_{n}(x)=0
$$

Rearranjando a equação anterior

$$
\frac{d^{2} \Psi_{n}(x)}{d x^{2}}-\left(\frac{2 m^{*} s_{n}}{\hbar^{2}}\right)\left[x+\left(\frac{V_{n}-E}{s_{n}}\right)\right] \Psi_{n}(x)=0
$$

Fazendo uma mudança de variáveis

$$
\rho_{n}(x)=\left(\frac{2 m^{*} s_{n}}{\hbar^{2}}\right)^{1 / 3}\left(x+\eta_{n}\right)
$$

onde

$$
\eta_{n}=\frac{V_{n}-E}{s_{n}}
$$

Com isso

$$
\begin{gathered}
\frac{d^{2} \Psi_{n}(x)}{d x^{2}}-\left(\frac{2 m^{*} s_{n}}{\hbar^{2}}\right)\left(x+\eta_{n}\right) \Psi_{n}(x)=0 \\
\frac{d^{2} \Psi_{n}(x)}{d x^{2}}-\left[\left(\frac{2 m^{*} s_{n}}{\hbar^{2}}\right)^{2 / 3}\left(\frac{2 m^{*} s_{n}}{\hbar^{2}}\right)^{1 / 3}\left(x+\eta_{n}\right)\right] \Psi_{n}(x)=0
\end{gathered}
$$

Escrevendo as derivadas em relação a nova mudança de variáveis

$$
\begin{gathered}
\frac{d}{d x} \rightarrow \frac{d}{d \rho_{n}} \frac{d \rho_{n}}{d x} \\
\frac{d^{2}}{d x^{2}} \rightarrow\left(\frac{d \rho_{n}}{d x}\right)^{2} \frac{d^{2}}{d \rho_{n}^{2}}=\left(\frac{2 m^{*} s_{n}}{\hbar^{2}}\right)^{2 / 3} \frac{d^{2}}{d \rho_{n}^{2}}
\end{gathered}
$$

Substituindo a Eq.(3.36) na Eq.(3.35)

$$
\left(\frac{2 m^{*} s_{n}}{\hbar^{2}}\right)^{2 / 3} \frac{d^{2} \Psi_{n}}{d \rho_{n}^{2}}-\left(\frac{2 m^{*} s_{n}}{\hbar^{2}}\right)^{2 / 3} \rho_{n} \Psi_{n}=0
$$

Manipulando a equação acima encontra-se a conhecida equação de Airy (equação de Bessel Modificada de ordem 1/3, Ref. [78]), cuja solução está na região 2,

$$
\frac{d^{2} \Psi_{2}}{d \rho_{2}^{2}}-\rho_{2} \Psi_{2}=0
$$


cuja a solução é a combinação linear das funções de Airy, $A i$ e $B i$, solução da região que possui o potencial inclinado devido a presença de um campo elétrico externo.

$$
\Psi_{2}(x)=C_{2} A i\left[\rho_{2}(x)\right]+D_{2} B i\left[\rho_{2}(x)\right]
$$

\section{Região 3:}

$$
-\frac{\hbar^{2}}{2 m_{3}^{*}} \frac{d^{2} \Psi_{3}(x)}{d x^{2}}=(q V+E) \Psi_{3}(x) \rightarrow \frac{d^{2} \Psi_{3}(x)}{d x^{2}}+\frac{2 m_{3}^{*}}{\hbar^{2}}(q V+E) \Psi_{3}(x)=0
$$

onde $k_{3}=\sqrt{\frac{2 m_{3}^{*}}{\hbar^{2}}(q V+E)}$

$$
\frac{d^{2} \Psi_{3}(x)}{d x^{2}}+k_{3}^{2} \Psi_{3}(x)=0
$$

Como solução da Eq.(3.40)

$$
\Psi_{3}(x)=C_{3} \exp \left(i k_{3} x\right)+D_{3} \exp \left(-i k_{3} x\right)
$$

Aplicando as condições de contorno

$$
\begin{gathered}
\Psi_{j}\left(x_{j}\right)=\Psi_{j+1}\left(x_{j}\right) \\
\frac{1}{m_{j}^{*}} \frac{d \Psi_{j}\left(x_{j}\right)}{d x}=\frac{1}{m_{j+1}^{*}} \frac{d \Psi_{j+1}\left(x_{j}\right)}{d x}
\end{gathered}
$$

Com a aplicação das condições de contorno, agora pode-se relacionar as amplitudes das ondas incidentes e transmitidas

$$
\begin{aligned}
{\left[\begin{array}{c}
C_{1} \\
D_{1}
\end{array}\right]=} & {\left[\begin{array}{cc}
\exp \left(i k_{1} x_{1}\right) & \exp \left(-i k_{1} x_{1}\right) \\
\frac{i k_{1}}{m_{1}^{*}} \exp \left(i k_{1} x_{1}\right) & -\frac{i k_{1}}{m_{1}^{*}} \exp \left(-i k_{1} x_{1}\right)
\end{array}\right]^{-1} \times } \\
& \times\left[\begin{array}{cc}
A i\left[\rho_{2}\left(x_{1}\right)\right] & B i\left[\rho_{2}\left(x_{1}\right)\right] \\
\frac{1}{m_{2}^{*}} A i^{\prime}\left[\rho_{2}\left(x_{1}\right)\right] & \frac{1}{m_{2}^{*}} B i^{\prime}\left[\rho_{2}\left(x_{1}\right)\right]
\end{array}\right] \times \\
& \times\left[\begin{array}{cc}
A i\left[\rho_{2}\left(x_{2}\right)\right] & B i\left[\rho_{2}\left(x_{2}\right)\right] \\
\frac{1}{m_{2}^{*}} A i^{\prime}\left[\rho_{2}\left(x_{2}\right)\right] & \frac{1}{m_{2}^{*}} B i^{\prime}\left[\rho_{2}\left(x_{2}\right)\right]
\end{array}\right] \times \\
& \times\left[\begin{array}{cc}
\exp \left(i k_{3} x_{2}\right) & \exp \left(-i k_{3} x_{2}\right) \\
\frac{i k_{3}}{m_{3}^{*}} \exp \left(i k_{3} x_{2}\right) & -\frac{i k_{3}}{m_{3}^{*}} \exp \left(-i k_{3} x_{2}\right)
\end{array}\right]\left[\begin{array}{c}
C_{3} \\
D_{3}
\end{array}\right]
\end{aligned}
$$


Efetuando o cálculo das matrizes inversas da Eq.(3.44), tem-se

$$
\begin{aligned}
{\left[\begin{array}{c}
C_{1} \\
D_{1}
\end{array}\right]=} & {\left[\begin{array}{cc}
\frac{1}{2} \exp \left(-i k_{1} x_{1}\right) & -\frac{i m_{1}^{*}}{2 k_{1}} \exp \left(-i k_{1} x_{1}\right) \\
\frac{1}{2} \exp \left(i k_{1} x_{1}\right) & -\frac{i k_{1}}{m_{1}^{*}} \exp \left(-i k_{1} x_{1}\right)
\end{array}\right] \times } \\
& \times\left[\begin{array}{cc}
A i\left[\rho_{2}\left(x_{1}\right)\right] & B i\left[\rho_{2}\left(x_{1}\right)\right] \\
\frac{1}{m_{2}^{*}} A i^{\prime}\left[\rho_{2}\left(x_{1}\right)\right] & \frac{1}{m_{2}^{*}} B i^{\prime}\left[\rho_{2}\left(x_{1}\right)\right]
\end{array}\right] \times \\
& \times\left[\begin{array}{cc}
\frac{1}{\mathbf{W}\left(A i\left[\rho_{2}\left(x_{2}\right)\right], B i\left[\rho_{2}\left(x_{2}\right)\right]\right)} B i^{\prime}\left[\rho_{2}\left(x_{2}\right)\right] & -\frac{m_{2}^{*}}{\mathbf{W}\left(A i\left[\rho_{2}\left(x_{2}\right)\right], B i\left[\rho_{2}\left(x_{2}\right)\right]\right)} B i\left[\rho_{2}\left(x_{2}\right)\right] \\
-\frac{1}{\mathbf{W}\left(A i\left[\rho_{2}\left(x_{2}\right)\right], B i\left[\rho_{2}\left(x_{2}\right)\right]\right)} A i^{\prime}\left[\rho_{2}\left(x_{2}\right)\right] & \frac{m_{2}^{*}}{\mathbf{W}\left(A i\left[\rho_{2}\left(x_{2}\right)\right], B i\left[\rho_{2}\left(x_{2}\right)\right]\right)} A i\left[\rho_{2}\left(x_{2}\right)\right]
\end{array}\right] \times \\
& \times\left[\begin{array}{cc}
\exp \left(i k_{3} x_{2}\right) & \exp \left(-i k_{3} x_{2}\right) \\
\frac{i k_{3}}{m_{3}^{*}} \exp \left(i k_{3} x_{2}\right) & -\frac{i k_{3}}{m_{3}^{*}} \exp \left(-i k_{3} x_{2}\right)
\end{array}\right]\left[\begin{array}{c}
C_{3} \\
D_{3}
\end{array}\right]
\end{aligned}
$$

onde $\mathbf{W}\left(A i\left[\rho_{2}\left(x_{2}\right)\right], B i\left[\rho_{2}\left(x_{2}\right)\right]\right)$ é o Wronskiano das funções de Airy, ou seja:

$$
\mathbf{W}\left(A i\left[\rho_{2}\left(x_{2}\right)\right], B i\left[\rho_{2}\left(x_{2}\right)\right]\right)=A i\left[\rho_{2}\left(x_{2}\right)\right] B i^{\prime}\left[\rho_{2}\left(x_{2}\right)\right]-A i^{\prime}\left[\rho_{2}\left(x_{2}\right)\right] B i\left[\rho_{2}\left(x_{2}\right)\right]
$$

Com a multiplicação das matrizes tem-se uma matriz resultante denominada $\mathbf{M}$,

$$
\mathbf{M}=\left[\begin{array}{ll}
\mathbf{M}_{11} & \mathbf{M}_{12} \\
\mathbf{M}_{21} & \mathbf{M}_{22}
\end{array}\right]
$$

Relacionando a matriz $\mathbf{M}$ com os coeficientes das funções de onda,

$$
\left[\begin{array}{l}
C_{1} \\
D_{1}
\end{array}\right]=\left[\begin{array}{ll}
\mathbf{M}_{11} & \mathbf{M}_{12} \\
\mathbf{M}_{21} & \mathbf{M}_{22}
\end{array}\right]\left[\begin{array}{c}
C_{3} \\
D_{3}
\end{array}\right]
$$

assumindo que $C_{1}$ seja igual a 1 , e a amplitude $D_{3}$ seja igual a zero, para que não haja reflexão na segunda interface em $x=x_{2}$.

$$
\left[\begin{array}{c}
1 \\
D_{1}
\end{array}\right]=\left[\begin{array}{ll}
\mathbf{M}_{11} & \mathbf{M}_{12} \\
\mathbf{M}_{21} & \mathbf{M}_{22}
\end{array}\right]\left[\begin{array}{c}
C_{3} \\
0
\end{array}\right]
$$

Agora, pode-se relacionar as amplitudes $C_{1}$ e $C_{3}$, e, assim, obter o coeficiente de transmissão

$$
\mathbf{T}=\left(\frac{k_{3} m_{1}^{*}}{k_{1} m_{3}^{*}}\right) \frac{\left|C_{3}\right|^{2}}{\left|C_{1}\right|^{2}}=\left(\frac{k_{3} m_{1}^{*}}{k_{1} m_{3}^{*}}\right) \frac{1}{\mathbf{M}_{11}^{*} \mathbf{M}_{11}}
$$


Após efetuar as multiplicações das matrizes, o que nos importa para o cálculo do coeficiente de transmissão é o elemento $\mathbf{M}_{11}$, que após algumas manipulações algébricas e simplificações, é dado por

$$
\begin{aligned}
\mathbf{M}_{11}= & \frac{\exp \left[i\left(k_{3} x_{2}-k_{1} x_{1}\right)\right]}{2 \mathbf{W}\left(A i\left[\rho_{2}\left(x_{2}\right)\right], B i\left[\rho_{2}\left(x_{2}\right)\right]\right)}\left\{\left[A i\left[\rho_{2}\left(x_{1}\right)\right] B i^{\prime}\left[\rho_{2}\left(x_{2}\right)\right]-B i\left[\rho_{2}\left(x_{1}\right)\right] A i^{\prime}\left[\rho_{2}\left(x_{2}\right)\right]\right]-\right. \\
& \left.-i\left(\frac{1}{k_{1}} \frac{m_{1}^{*}}{m_{2}^{*}}\right)\left[A i^{\prime}\left[\rho_{2}\left(x_{1}\right)\right] B i^{\prime}\left[\rho_{2}\left(x_{2}\right)\right]-B i^{\prime}\left[\rho_{2}\left(x_{1}\right)\right] A i^{\prime}\left[\rho_{2}\left(x_{2}\right)\right]\right]\right\}+ \\
& +\frac{\exp \left[i\left(k_{3} x_{2}-k_{1} x_{1}\right)\right]}{2 \mathbf{W}\left(A i\left[\rho_{2}\left(x_{2}\right)\right], B i\left[\rho_{2}\left(x_{2}\right)\right]\right)}\left(i \frac{k_{3}}{m_{3}^{*}} m_{2}^{*}\right)\left\{\left[-A i\left[\rho_{2}\left(x_{1}\right)\right] B i\left[\rho_{2}\left(x_{2}\right)\right]\right.\right. \\
& \left.+B i\left[\rho_{2}\left(x_{1}\right)\right] A i\left[\rho_{2}\left(x_{2}\right)\right]\right]-i\left(\frac{1}{k_{1}} \frac{m_{1}^{*}}{m_{2}^{*}}\right)\left[B i^{\prime}\left[\rho_{2}\left(x_{1}\right)\right] A i\left[\rho_{2}\left(x_{2}\right)\right]\right. \\
& \left.\left.-A i^{\prime}\left[\rho_{2}\left(x_{1}\right)\right] B i\left[\rho_{2}\left(x_{2}\right)\right]\right]\right\}
\end{aligned}
$$

Para facilitar a visualização, sugere-se algumas mudanças de variáveis:

$$
\begin{aligned}
\delta & =\left[A i\left[\rho_{2}\left(x_{1}\right)\right] B i^{\prime}\left[\rho_{2}\left(x_{2}\right)\right]-B i\left[\rho_{2}\left(x_{1}\right)\right] A i^{\prime}\left[\rho_{2}\left(x_{2}\right)\right]\right] \\
\sigma & =\left[A i^{\prime}\left[\rho_{2}\left(x_{1}\right)\right] B i^{\prime}\left[\rho_{2}\left(x_{2}\right)\right]-B i^{\prime}\left[\rho_{2}\left(x_{1}\right)\right] A i^{\prime}\left[\rho_{2}\left(x_{2}\right)\right]\right] \\
\chi & =\left[-A i\left[\rho_{2}\left(x_{1}\right)\right] B i\left[\rho_{2}\left(x_{2}\right)\right]+B i\left[\rho_{2}\left(x_{1}\right)\right] A i\left[\rho_{2}\left(x_{2}\right)\right]\right] \\
\gamma & =\left[A i^{\prime}\left[\rho_{2}\left(x_{1}\right)\right] B i\left[\rho_{2}\left(x_{2}\right)\right]-B i^{\prime}\left[\rho_{2}\left(x_{1}\right)\right] A i\left[\rho_{2}\left(x_{2}\right)\right]\right]
\end{aligned}
$$

Com isso pode-se reescrever $\mathbf{M}_{11}$,

$$
\mathbf{M}_{11}=\frac{\exp \left[i\left(k_{3} x_{2}-k_{1} x_{1}\right)\right]}{2 \mathbf{W}\left(A i\left[\rho_{2}\left(x_{2}\right)\right], B i\left[\rho_{2}\left(x_{2}\right)\right]\right)}\left\{\left[\delta-i\left(\frac{1}{k_{1}} \frac{m_{1}^{*}}{m_{2}^{*}}\right) \sigma\right]+\left(i \frac{k_{3}}{m_{3}^{*}} m_{2}^{*}\right)\left[\chi+i\left(\frac{1}{k_{1}} \frac{m_{1}^{*}}{m_{2}^{*}}\right) \gamma\right]\right\}
$$

Reagrupando

$$
\mathbf{M}_{11}=\frac{\exp \left[i\left(k_{3} x_{2}-k_{1} x_{1}\right)\right]}{2 \mathbf{W}\left(A i\left[\rho_{2}\left(x_{2}\right)\right], B i\left[\rho_{2}\left(x_{2}\right)\right]\right)}\left\{\delta-i\left(\frac{1}{k_{1}} \frac{m_{1}^{*}}{m_{2}^{*}}\right) \sigma+i\left(\frac{k_{3}}{m_{3}^{*}} m_{2}^{*}\right) \chi-\left(\frac{k_{3}}{k_{1}} \frac{m_{1}^{*}}{m_{3}^{*}}\right) \gamma\right\}
$$

Rearranjando

$$
\mathbf{M}_{11}=\frac{\exp \left[i\left(k_{3} x_{2}-k_{1} x_{1}\right)\right]}{2 \mathbf{W}\left(A i\left[\rho_{2}\left(x_{2}\right)\right], B i\left[\rho_{2}\left(x_{2}\right)\right]\right)}\left\{\left[\delta-\left(\frac{k_{3}}{k_{1}} \frac{m_{1}^{*}}{m_{3}^{*}}\right) \gamma\right]+i\left[\left(\frac{k_{3}}{m_{3}^{*}} m_{2}^{*}\right) \chi-\left(\frac{1}{k_{1}} \frac{m_{1}^{*}}{m_{2}^{*}}\right) \sigma\right]\right\}
$$


Calculando o complexo conjugado do primeiro elemento $\mathbf{M}_{11}^{*}$

$$
\begin{aligned}
\mathbf{M}_{11}^{*}= & \frac{\exp \left[-i\left(k_{3} x_{2}-k_{1} x_{1}\right)\right]}{2 \mathbf{W}^{*}\left(A i\left[\rho_{2}\left(x_{2}\right)\right], B i\left[\rho_{2}\left(x_{2}\right)\right]\right)} \times \\
& \times\left\{\left[\delta-\left(\frac{k_{3}}{k_{1}} \frac{m_{2}^{*}}{m_{3}^{*}} \frac{m_{1}^{*}}{m_{2}^{*}}\right) \gamma\right]^{*}-i\left[\left(\frac{k_{3}}{m_{3}^{*}} m_{2}^{*}\right) \chi-\left(\frac{1}{k_{1}} \frac{m_{1}^{*}}{m_{2}^{*}}\right) \sigma\right]^{*}\right\}
\end{aligned}
$$

Efetuando a multiplicação $\mathbf{M}_{11}^{*} \mathbf{M}_{11}$

$$
\begin{aligned}
\mathbf{M}_{11}^{*} \mathbf{M}_{11}= & \frac{1}{4 \mathbf{W}^{*}\left(A i\left[\rho_{2}\left(x_{2}\right)\right], B i\left[\rho_{2}\left(x_{2}\right)\right]\right) \mathbf{W}\left(A i\left[\rho_{2}\left(x_{2}\right)\right], B i\left[\rho_{2}\left(x_{2}\right)\right]\right)} \times \\
& \times\left\{\left[\delta-\left(\frac{k_{3}}{k_{1}} \frac{m_{2}^{*}}{m_{3}^{*}} \frac{m_{1}^{*}}{m_{2}^{*}}\right) \gamma\right]^{*}-i\left[\left(\frac{k_{3}}{m_{3}^{*}} m_{2}^{*}\right) \chi-\left(\frac{1}{k_{1}} \frac{m_{1}^{*}}{m_{2}^{*}}\right) \sigma\right]^{*}\right\} \times \\
& \times\left\{\left[\delta-\left(\frac{k_{3}}{k_{1}} \frac{m_{1}^{*}}{m_{3}^{*}}\right) \gamma\right]+i\left[\left(\frac{k_{3}}{m_{3}^{*}} m_{2}^{*}\right) \chi-\left(\frac{1}{k_{1}} \frac{m_{1}^{*}}{m_{2}^{*}}\right) \sigma\right]\right\} \\
\mathbf{M}_{11}^{*} \mathbf{M}_{11}= & \frac{1}{4\left|\mathbf{W}\left(A i\left[\rho_{2}\left(x_{2}\right)\right], B i\left[\rho_{2}\left(x_{2}\right)\right]\right)\right|^{2}} \times \\
& \times\left\{\left|\delta-\left(\frac{k_{3}}{k_{1}} \frac{m_{1}^{*}}{m_{3}^{*}}\right) \gamma\right|^{2}+\left|\left(\frac{k_{3}}{m_{3}^{*}} m_{2}^{*}\right) \chi-\left(\frac{1}{k_{1}} \frac{m_{1}^{*}}{m_{2}^{*}}\right) \sigma\right|^{2}\right\}
\end{aligned}
$$

sabendo que $\mathbf{W}\left(A i\left[\rho_{2}\left(x_{2}\right)\right], B i\left[\rho_{2}\left(x_{2}\right)\right]\right)=\pi^{-1}$, Ref. [78], então

$$
\mathbf{M}_{11}^{*} \mathbf{M}_{11}=\frac{\pi^{2}}{4}\left\{\left|\delta-\left(\frac{k_{3}}{k_{1}} \frac{m_{1}^{*}}{m_{3}^{*}}\right) \gamma\right|^{2}+\left|\left(\frac{k_{3}}{m_{3}^{*}} m_{2}^{*}\right) \chi-\left(\frac{1}{k_{1}} \frac{m_{1}^{*}}{m_{2}^{*}}\right) \sigma\right|^{2}\right\}
$$

Assumindo que $m_{1}^{*}=m_{3}^{*}$ e $k_{1}=k_{3}$, tem-se

$$
\mathbf{M}_{11}^{*} \mathbf{M}_{11}=\frac{\pi^{2}}{4}\left\{|\delta-\gamma|^{2}+\left|\left(\frac{k_{3}}{m_{3}^{*}} m_{2}^{*}\right) \chi-\left(\frac{1}{k_{1}} \frac{m_{1}^{*}}{m_{2}^{*}}\right) \sigma\right|^{2}\right\}
$$

Com isso tem-se o coeficiente de transmissão, substituindo a Eq.(3.59) na Eq.(3.49)

$$
\mathbf{T}(E, V)=\frac{1}{\mathbf{M}_{11}^{*} \mathbf{M}_{11}}=\frac{4}{\pi^{2}\left\{|\delta-\gamma|^{2}+\left|\left(\frac{k_{3}}{m_{3}^{*}} m_{2}^{*}\right) \chi-\left(\frac{1}{k_{1}} \frac{m_{1}^{*}}{m_{2}^{*}}\right) \sigma\right|^{2}\right\}}
$$

Enfim, obtém-se o coeficiente de transmissão de uma barreira de potencial com polarização, de forma analítica, em função da tensão aplicada.

Como pode ser visto, o problema que relacionado à uma polarização aplicada, $V$, em uma barreira de potencial, apresenta a solução na região da barreira em termos de combinações 
lineares das funções de Airy: $A i$ e $B i$. Com isso o cálculo do coeficiente de transmissão em função da energia da partícula incidente e da tensão aplicada, $V$, representada pela Eq.(3.60) mostra um resultado dificil de ser manipulado matematicamente, evidenciando que este método tornar-se-a cada vez mais complicado à medida que o número n de barreiras do sistema aumenta. Para superar essa dificuldade é apresentado na Seção (3.2.1) um modelo denominado na literatura por "Step Approximation"[79], o qual diminui enormemente a dificuldade de se encontrar o coeficiente de transmissão, $\mathbf{T}(E, V)$ com relação ao método analítico. O método pode ser estendido para outras super-heteroestruturas desde que as equações utilizadas sejam readaptadas para o número desejado de barreiras.

\section{Solução numérica de acordo com M. Abe et al.[79]}

Devido às dificuldades que se verificam na solução da equação de Schrodinger para estruturas com um número arbitrário de barreiras de potencial sujeitas a aplicação de uma polarização, é apresentada nesta seção uma aproximação conhecida na literatura como "Step Approximation" [79], a qual consite em dividir a região delimitada pela função potencial $V(x)$ em vários retângulos de comprimento $d x$ e altura $V(x)$, particionando a figura como mostrada na Fig.(3.3). Em seguida, pode-se aplicar o Método da Matriz de Transferência (MMT), apresentada anteriormente na Seção (3.2.4), para cada barreira, individualmente, analisando-se a Fig.(3.3).

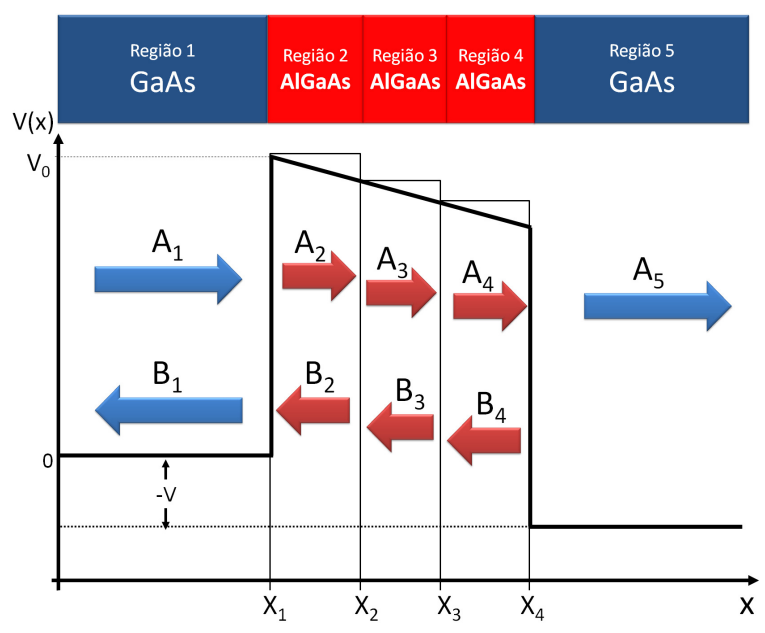

Figura 3.3: Perfil de potencial de uma barreira de potencial finito com polarização aplicada, utilizando as divisões da barreira inclinada, método denominado "Step Approximation", Ref. [79]. 
A Fig.(3.3) mostra cinco regiões, para as quais resolve-se a equação de Schrödinger, individualmente.

\section{Região 1:}

A equação de Schrödinger para a Região 1

$$
\frac{d^{2} \Psi_{1}(x)}{d x^{2}}+\frac{2 m_{1}^{*}}{\hbar^{2}} E \Psi_{1}(x)=0
$$

onde $k_{1}=\sqrt{\frac{2 m_{1}^{*}}{\hbar^{2}} E}$

$$
\frac{d^{2} \Psi_{1}(x)}{d x^{2}}+k_{1}^{2} \Psi_{1}(x)=0
$$

Como solução tem-se

$$
\Psi_{1}(x)=A_{1} \exp \left(i k_{1} x\right)+B_{1} \exp \left(-i k_{1} x\right)
$$

\section{Região 2:}

$$
\frac{d^{2} \Psi_{2}(x)}{d x^{2}}-\frac{2 m_{2}^{*}}{\hbar^{2}}\left(V_{0}-E\right) \Psi_{2}(x)=0 \rightarrow
$$

onde $k_{2}=\sqrt{\frac{2 m_{2}^{*}}{\hbar^{2}}\left(V_{0}-E\right)}$

$$
\frac{d^{2} \Psi_{2}(x)}{d x^{2}}-k_{2}^{2} \Psi_{2}(x)=0
$$

Como solução tem-se

$$
\Psi_{2}(x)=A_{2} \exp \left(k_{2} x\right)+B_{2} \exp \left(-k_{2} x\right)
$$

\section{Região 3:}

$$
\frac{d^{2} \Psi_{3}(x)}{d x^{2}}-\frac{2 m_{3}^{*}}{\hbar^{2}}\left(V_{0}-\frac{q V}{3}-E\right) \Psi_{3}(x)=0 \rightarrow
$$

onde $k_{3}=\sqrt{\frac{2 m_{3}^{*}}{\hbar^{2}}\left(V_{0}-\frac{q V}{3}-E\right)}$

$$
\frac{d^{2} \Psi_{3}(x)}{d x^{2}}+k_{3}^{2} \Psi_{3}(x)=0
$$

Como solução tem-se 


$$
\Psi_{3}(x)=A_{3} \exp \left(k_{3} x\right)+B_{3} \exp \left(-k_{3} x\right)
$$

\section{Região 4:}

$$
\frac{d^{2} \Psi_{4}(x)}{d x^{2}}-\frac{2 m_{4}^{*}}{\hbar^{2}}\left(V_{0}-\frac{2 q V}{3}-E\right) \Psi_{4}(x)=0
$$

onde $k_{4}=\sqrt{\frac{2 m_{4}^{*}}{\hbar^{2}}\left(V_{0}-\frac{2 q V}{3}-E\right)}$

$$
\frac{d^{2} \Psi_{4}(x)}{d x^{2}}-k_{4}^{2} \Psi_{4}(x)=0
$$

Como solução tem-se

$$
\Psi_{4}(x)=A_{4} \exp \left(k_{4} x\right)+B_{4} \exp \left(-k_{4} x\right)
$$

\section{Região 5:}

$$
\frac{d^{2} \Psi_{5}(x)}{d x^{2}}+\frac{2 m_{5}^{*}}{\hbar^{2}}(E+q V) \Psi_{5}(x)=0
$$

onde $k_{5}=\sqrt{\frac{2 m_{5}^{*}}{\mathrm{\hbar}^{2}}(E+q V)}$

$$
\frac{d^{2} \Psi_{5}(x)}{d x^{2}}+k_{5}^{2} \Psi_{5}(x)=0
$$

Como solução tem-se

$$
\Psi_{5}(x)=A_{5} \exp \left(i k_{5} x\right)+B_{5} \exp \left(-i k_{5} x\right)
$$

Quando $E>V_{0}$, então $k_{1}$ e $k_{2}$ são reais e as funções de onda serão ondas planas. Quando $E<V_{0}$, então o $k_{2}$ será puramente imaginário, tem-se funções de onda em decaimento exponencial. As condições de contornos obtidas a partir das Eqs.(3.42) e (3.43) determinam os coeficientes $A$ e $B$ através da matriz $R$

$$
R=\frac{1}{2 k_{1} m_{2}}\left[\begin{array}{cc}
\left(k_{1} m_{2}+k_{2} m_{1}\right) \exp \left[i\left(k_{2}-k_{1}\right) x_{1}\right] & \left(k_{1} m_{2}-k_{2} m_{1}\right) \exp \left[-i\left(k_{2}+k_{1}\right) x_{1}\right] \\
\left(k_{1} m_{2}-k_{2} m_{1}\right) \exp \left[i\left(k_{2}+k_{1}\right) x_{1}\right] & \left(k_{1} m_{2}+k_{2} m_{1}\right) \exp \left[-i\left(k_{2}-k_{1}\right) x_{1}\right]
\end{array}\right]
$$

Aplicando a matriz $R$, nas interfaces, temos:

$$
\left[\begin{array}{c}
A_{1} \\
B_{1}
\end{array}\right]=R_{1} \cdot R_{2} \cdot R_{3} \cdot R_{4}\left[\begin{array}{c}
A_{5} \\
B_{5}
\end{array}\right]
$$




$$
\left[\begin{array}{l}
A_{1} \\
B_{1}
\end{array}\right]=\left[\begin{array}{ll}
\mathbf{M}_{11} & \mathbf{M}_{12} \\
\mathbf{M}_{21} & \mathbf{M}_{22}
\end{array}\right]\left[\begin{array}{l}
A_{5} \\
B_{5}
\end{array}\right]
$$

Assumindo que $B_{5}$ seja igual a zero, considerando que não há reflexão na quarta interface e utilizando o termo da matriz de transferência $\mathbf{M}_{11}$, que corresponde ao primeiro elemento da multiplicação das matrizes $R_{1} \cdot R_{2} \cdot R_{3} \cdot R_{4}$ tem-se

$$
A_{1}=\mathbf{M}_{11} A_{5}
$$

O coeficiente de transmissão, T, obtido através da aproximação do método dos retângulos, usando, número de retângulos, $N=3$ é mostrado na Eq.(3.74)

$$
\begin{gathered}
\mathbf{T}=\left(\frac{k_{5} m_{1}^{*}}{k_{1} m_{5}^{*}}\right) \frac{\left|A_{5}\right|^{2}}{\left|A_{1}\right|^{2}} \\
\mathbf{T}=\left(\frac{k_{5} m_{1}^{*}}{k_{1} m_{5}^{*}}\right) \frac{A_{5}^{*} A_{5}}{A_{1}^{*} A_{1}}=\left(\frac{k_{5} m_{1}^{*}}{k_{1} m_{5}^{*}}\right) \frac{1}{\mathbf{M}_{11}^{*} \mathbf{M}_{11}}
\end{gathered}
$$

Por indução, o resultado obtido para o cálculo do coeficiente de transmissão, T, usandose o número $N$ de retângulos numa estrutura com uma única barreira de potencial, sujeita à aplicação de uma tensão externa, $V$, pode ser escrito como

$$
\mathbf{T}=\left(\frac{k_{2 N-1} m_{1}^{*}}{k_{1} m_{2 N-1}^{*}}\right) \frac{A_{2 N-1}^{*} A_{2 N-1}}{A_{1}^{*} A_{1}}=\left(\frac{k_{2 N-1} m_{1}^{*}}{k_{1} m_{2 N-1}^{*}}\right) \frac{1}{\mathbf{M}_{11}^{*} \mathbf{M}_{11}}
$$

A Fig.(3.4) mostra o comportamento do logarítmo do coeficiente de transmissão fornecido pela Eq.(3.75) para vários valores de N compreendidos entre 1 e 100, num mesmo gráfico. Podese observar que, para $N>30$ o resultado obtido para o coeficiente de transmissão não mais varia significativamente, convergindo para o resultado esperado da Ref. [54]. De fato, pode-se concluir que, a aproximação pelo método do "Step Approximation"simplifica os cálculos computacionais, evitando-se as complicações do tratamento analítico. No entanto, o método precisa ser readaptado para o cálculo do coeficiente de transmissão para o caso de uma estrutura com n barreiras, levando a resultados satisfatórios quando contrastadas com a literatura. Porém, o cálculo computacional vai se tornando mais pesado à medida que o número de barreiras aumentam. Sendo assim, um melhoramento neste método foi proposto inicialmente por Levi [49], que demonstra que a aproximação dos métodos dos retângulos pode ser utilizada em toda a extensão de um perfil de potencial arbitrário $V(x)$, como pode ser visto na próxima seção. 


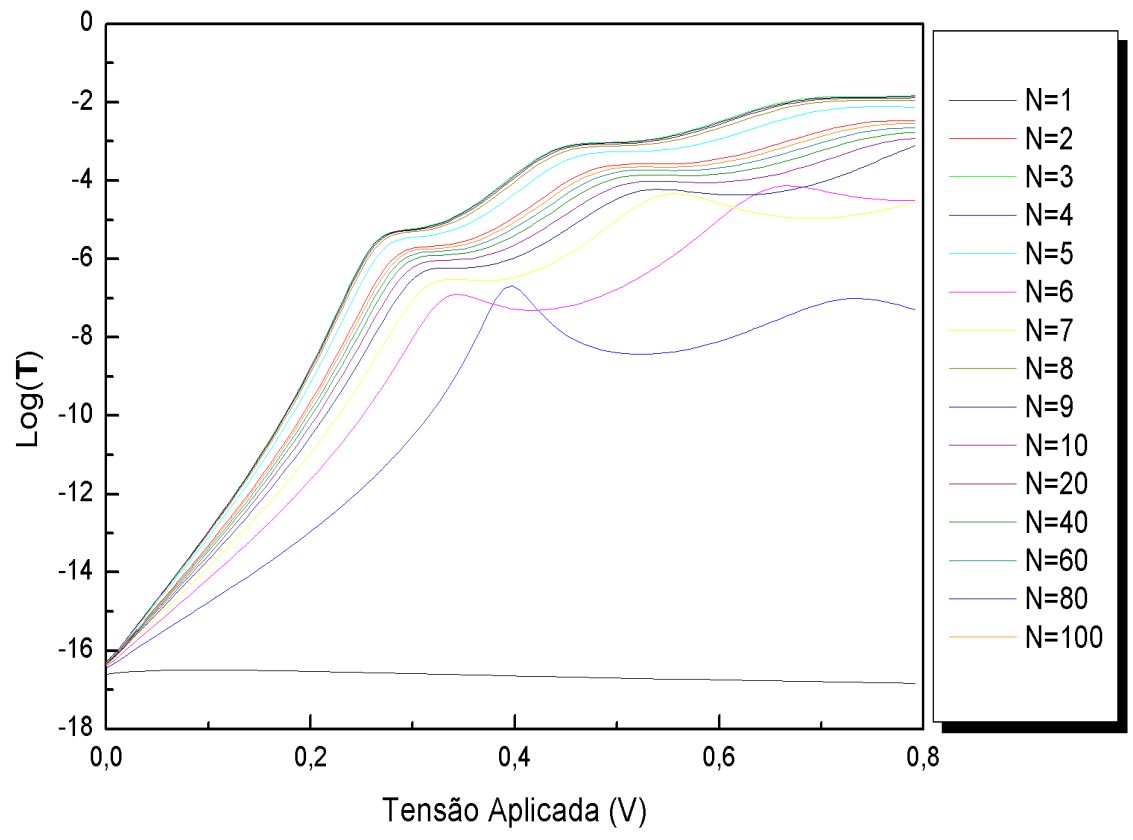

Figura 3.4: Logaritmo do coeficiente de transmissão em função da tensão aplicada em função do número $N$ de retângulos, usando Método dos Retângulos, para $E=0,05 \mathrm{eV}$, de acordo com a Ref. [54].

\section{Solução numérica de acordo com Levi [49]}

Um melhoramento na aproximação utilizada na Seção (3.2.1) pode ser obtido considerandose a utilização do que será denominado neste trabalho de aproximação do método dos retângulos (AMR) por toda extensão do perfil de potencial $V(x)$, como mostrado na Fig.(3.5).

Esta metodologia é usada por Levi na Ref. [49], na qual considera-se não somente a região da barreira, mas todo o perfil de potencial $V(x)$, mostrado na Fig.(3.5), dividindo-o em $\mathrm{N}$ retângulos, onde cada retângulo tem largura $d x=1 \AA$.

Na Fig.(3.5) pode-se distinguir dois tipos de situações para o perfil de potencial, aquela ilustrada no quadrado indicado com a letra (a), e uma outra situação, como a indicada pela letra (b). A situação ilustrada em (a) mostra o pelfil de potencial representado por um potencial inclinado, enquanto que, a situação em (b) mostra o perfil de potencial indicado por uma reta paralela ao eixo $x$. Sendo assim, separando-se ambas as regiões, pode-se inicialmente resolver a equação de Schrödinger na situação (a) da Fig.(3.5), para uma região $j$ qualquer, escrevendo-se 


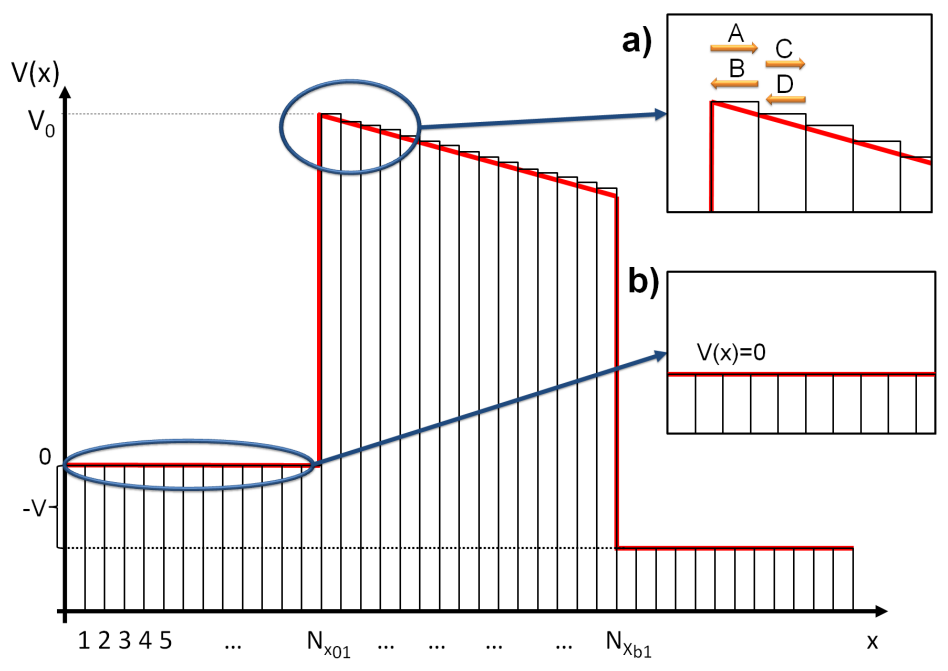

Figura 3.5: Esquema de uma barreira de potencial com polarização aplicada: a) representa uma visualização ampliada da região com a inclinação do potencial, região, da barreira; b) região paralela ao eixo $\mathrm{x}$.

$$
\begin{gathered}
-\frac{\hbar^{2}}{2 m_{j}^{*}} \frac{d^{2} \Psi_{j}(x)}{d x^{2}}+V_{j}(x) \Psi_{j}(x)=E \Psi_{j}(x) \\
\frac{d^{2} \Psi_{j}(x)}{d x^{2}}+\frac{2 m_{j}^{*}}{\hbar^{2}}\left(E-V_{j}(x)\right) \Psi_{j}(x)=0 \\
\frac{d^{2} \Psi_{j}(x)}{d x^{2}}+k_{j}^{2} \Psi_{j}(x)=0
\end{gathered}
$$

no qual,

$$
k_{j}=\sqrt{\frac{2 m_{j}^{*}}{\hbar^{2}}\left(E-V_{j}(x)\right)}
$$

A partir da Eq.(3.77), pode-se escrever a solução da equação de Schrödinger para as regiões $j$ e $j+1$ da forma

$$
\begin{gathered}
\Psi_{j}=A_{j} e^{i k_{j} x}+B_{j} e^{-i k_{j} x} \\
\Psi_{j+1}=C_{j+1} e^{i k_{j+1} x}+D_{j+1} e^{-i k_{j+1} x}
\end{gathered}
$$

Impondo-se as condições de continuidade na interface, em $x=x_{j+1}$, como usual tem-se

$$
\Psi_{j}\left(x=x_{j+1}\right)=\Psi_{j+1}\left(x=x_{j+1}\right)
$$




$$
\frac{1}{m_{j}} \frac{d}{d x} \Psi_{j}\left(x=x_{j+1}\right)=\frac{1}{m_{j+1}} \frac{d}{d x} \Psi_{j+1}\left(x=x_{j+1}\right)
$$

Substituindo as Eqs.(3.79) e (3.80), nas condições (3.81) e (3.82) obtém-se

$$
\begin{gathered}
A_{j} e^{i k_{j} x_{j+1}}+B_{j} e^{-i k_{j} x_{j+1}}=C_{j+1} e^{i k_{j+1} x_{j+1}}+D_{j+1} e^{-i k_{j+1} x_{j+1}} \\
A_{j} e^{i k_{j} x_{j+1}}-B_{j} e^{-i k_{j} x_{j+1}}=\frac{m_{j}}{m_{j+1}} \frac{k_{j+1}}{k_{j}}\left[C_{j+1} e^{i k_{j+1} x_{j+1}}-D_{j+1} e^{-i k_{j+1} x_{j+1}}\right]
\end{gathered}
$$

Agora pode-se escrever as Eqs.(3.83) e (3.84), em forma matricial na posição $x_{j+1}=0$.

$$
\left[\begin{array}{cc}
1 & 1 \\
1 & -1
\end{array}\right]\left[\begin{array}{c}
A_{j} \\
B_{j}
\end{array}\right]=\left[\begin{array}{cc}
1 & 1 \\
\frac{m_{j}}{m_{j+1}} \frac{k_{j+1}}{k_{j}} & -\frac{m_{j}}{m_{j+1}} \frac{k_{j+1}}{k_{j}}
\end{array}\right]\left[\begin{array}{c}
C_{j+1} \\
D_{j+1}
\end{array}\right]
$$

Portanto, a inversa de $\left[\begin{array}{cc}1 & 1 \\ 1 & -1\end{array}\right]$ é $\frac{1}{2}\left[\begin{array}{cc}1 & 1 \\ 1 & -1\end{array}\right]$, agora podemos escrever:

$$
\left[\begin{array}{c}
A_{j} \\
B_{j}
\end{array}\right]=\frac{1}{2}\left[\begin{array}{cc}
1 & 1 \\
1 & -1
\end{array}\right]\left[\begin{array}{cc}
1 & 1 \\
\frac{m_{j}}{m_{j+1}} \frac{k_{j+1}}{k_{j}} & -\frac{m_{j}}{m_{j+1}} \frac{k_{j+1}}{k_{j}}
\end{array}\right]\left[\begin{array}{c}
C_{j+1} \\
D_{j+1}
\end{array}\right]
$$

onde a matriz de propagação entre retângulos no caso da polarização aplicada, será

$$
\left[\begin{array}{c}
A_{j} \\
B_{j}
\end{array}\right]=\mathbf{R}_{j_{r e t}}\left[\begin{array}{c}
C_{j+1} \\
D_{j+1}
\end{array}\right]
$$

no qual $\mathbf{R}_{j_{r e t}}$ é uma matriz $2 \times 2$ que descreve as ondas de propagação entre os retângulos com polarização aplicada.

Então

$$
\mathbf{R}_{j_{\text {ret }}}=\frac{1}{2}\left[\begin{array}{cc}
1+\left(\frac{m_{j}}{m_{j+1}} \frac{k_{j+1}}{k_{j}}\right) & 1-\left(\frac{m_{j}}{m_{j+1}} \frac{k_{j+1}}{k_{j}}\right) \\
1-\left(\frac{m_{j}}{m_{j+1}} \frac{k_{j+1}}{k_{j}}\right) & 1+\left(\frac{m_{j}}{m_{j+1}} \frac{k_{j+1}}{k_{j}}\right)
\end{array}\right]
$$

Este resultado será utilizado mais adiante para descrever toda a estrutura estudada, de modo a finalizar a construção do Método da Matriz de Transferência para barreiras com polarização aplicada.

Para a situação (b) da Fig.(3.5), na qual o potencial é nulo, temos uma região sem polarização, com um comprimento $L_{j}=N-N_{x_{b}}$, em que as soluções da equação de Schrödinger se relacionam através dos seguintes coeficientes

$$
A_{j} e^{i k_{j} L_{j}}=C_{j+1}
$$




$$
B_{j} e^{-i k_{j} L_{j}}=D_{j+1}
$$

Na forma matricial tem-se

$$
\left[\begin{array}{cc}
e^{i k_{j} L_{j}} & 0 \\
0 & e^{-i k_{j} L_{j}}
\end{array}\right]\left[\begin{array}{c}
A_{j} \\
B_{j}
\end{array}\right]=\left[\begin{array}{c}
C_{j+1} \\
D_{j+1}
\end{array}\right]
$$

cuja inversa $\left[\begin{array}{cc}e^{i k_{j} L_{j}} & 0 \\ 0 & e^{-i k_{j} L_{j}}\end{array}\right]$ será

$$
\left[\begin{array}{cc}
e^{-i k_{j} L_{j}} & 0 \\
0 & e^{i k_{j} L_{j}}
\end{array}\right]
$$

Assim a forma matricial (3.91) pode ser escrita, como

$$
\left[\begin{array}{c}
A_{j} \\
B_{j}
\end{array}\right]=\left[\begin{array}{cc}
e^{-i k_{j} L_{j}} & 0 \\
0 & e^{i k_{j} L_{j}}
\end{array}\right]\left[\begin{array}{c}
C_{j+1} \\
D_{j+1}
\end{array}\right]=\mathbf{R}_{j_{\text {livre }}}\left[\begin{array}{c}
C_{j+1} \\
D_{j+1}
\end{array}\right]
$$

em que

$$
\mathbf{R}_{j_{\text {livre }}}=\left[\begin{array}{cc}
e^{-i k_{j} L_{j}} & 0 \\
0 & e^{i k_{j} L_{j}}
\end{array}\right]
$$

Com este resultado pode-se combinar a matriz $\mathbf{R}_{j_{\text {ret }}}$ com a matriz $\mathbf{R}_{j_{\text {livre }}}$, para com isso obter o MMT para barreiras com polarização aplicada.

Multiplicando as matrizes $\mathbf{R}_{j_{r e t}}$ e $\mathbf{R}_{j_{l i v r e}}$, então encontramos a propagação para qualquer região $j$ que será definido pela expressão

$$
\mathbf{R}_{j}=\mathbf{R}_{j_{r e t}} \mathbf{R}_{j_{\text {livre }}}=\left[\begin{array}{cc}
R_{1,1} & R_{1,2} \\
R_{2,1} & R_{2,2}
\end{array}\right]
$$

em que

$$
\mathbf{R}_{j}=\frac{1}{2}\left[\begin{array}{cc}
\left(1+\frac{m_{j}}{m_{j+1}} \frac{k_{j+1}}{k_{j}}\right) e^{-i k_{j} L_{j}} & \left(1-\frac{m_{j}}{m_{j+1}} \frac{k_{j+1}}{k_{j}}\right) e^{-i k_{j} L_{j}} \\
\left(1-\frac{m_{j}}{m_{j+1}} \frac{k_{j+1}}{k_{j}}\right) e^{i k_{j} L_{j}} & \left(1+\frac{m_{j}}{m_{j+1}} \frac{k_{j+1}}{k_{j}}\right) e^{i k_{j} L_{j}}
\end{array}\right]
$$

cujo resultado nos dá a matriz de propagação para a $j$ - ésima região [49]. 
Considerando-se a discretização uniforme com $N$ retângulos de largura de $1 \AA$ e escrevendose a matriz de propagação para cada região separadamente e multiplicando as matrizes, pode-se finalmente formular o MMT:

$$
\mathbf{R}=\mathbf{R}_{\mathbf{1}} \mathbf{R}_{\mathbf{2}} \ldots \mathbf{R}_{\mathbf{j}} \ldots \mathbf{R}_{N}=\prod_{j=1}^{N} \mathbf{R}_{\mathbf{j}}
$$

Agora temos

$$
\left[\begin{array}{l}
A \\
B
\end{array}\right]=\left(\prod_{j=1}^{N} \mathbf{R}_{\mathbf{j}}\right)\left[\begin{array}{l}
C \\
D
\end{array}\right]=\mathbf{R}\left[\begin{array}{l}
C \\
D
\end{array}\right]
$$

Assumindo que a partícula incide à partir da esquerda, tem-se que, $A=1$, e se não houver reflexão na saída da estrutura do lado direito, $D=0$.

$$
\left[\begin{array}{l}
1 \\
B
\end{array}\right]=\left[\begin{array}{ll}
R_{1,1} & R_{1,2} \\
R_{2,1} & R_{2,2}
\end{array}\right]\left[\begin{array}{l}
C \\
0
\end{array}\right]
$$

Neste caso, temos $1=\left(R_{1,1}\right) C$, e o coeficiente de transmissão, $\mathbf{T}$ é simplesmente

$$
\mathbf{T}=|C|^{2}=\left|\frac{1}{R_{1,1}}\right|^{2}
$$

A Eq.(3.99) é utilizada para calcular o coeficiente de transmissão de uma partícula através de uma barreira de potencial unidimensional com polarização aplicada. Para finalizar a formulação do método necessitamos conhecer o potencial $V(x)$ para as barreiras de potencial aplicada um campo elétrico externo.

Para obter o perfil de potencial $V(x)$ utilizou-se uma construção geométrica como mostrada na Fig.(3.6), a qual permite-nos levar em conta a inclinação $\alpha$ do perfil de potencial em cada ponto, dada por

$$
\alpha=-\frac{V}{\left(N_{x_{b}}-N_{x_{0}}\right)}
$$

e considerando o ponto da reta que equivale o potencial

$$
\left(x_{0}, y_{0}\right) \equiv\left(N_{x_{0}}, V_{0}\right)
$$

Usando-se a definição usual de equação da reta 


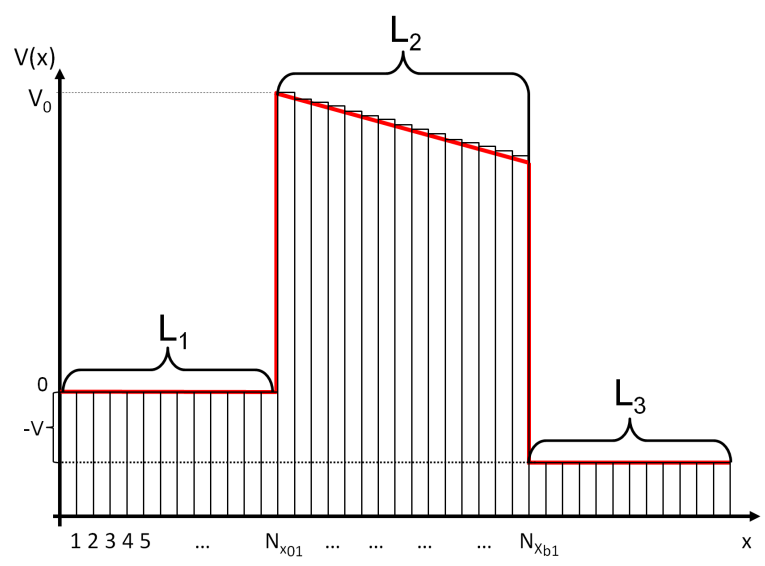

Figura 3.6: Esquema de uma barreira de potencial com polarização aplicada, divida em $N$ retângulos, evidenciando os comprimentos: $L_{1}, L_{2}$ e $L_{3}$.

$$
y-y_{0}=\alpha\left(x-x_{0}\right)
$$

tem-se o potencial $V(x)$ em função de $x$, substituindo as Eqs.(3.100) e (3.101) na Eq.(3.102), escrito como

$$
V(x)=V_{0}-\frac{V}{\left(N_{x_{b}}-N_{x_{0}}\right)}\left(x-N_{x_{0}}\right)
$$

Agora de posse de $V(x)$, pode-se substituir na Eq.(3.78) e com isso pode-se finalizar o formalismo do método da matriz de transferência para barreiras com polarização aplicada.

O melhoramento na construção deste método em relação ao apresentado na Seção (3.2.1), pemite que o método seja usado agora para um perfil de potencial arbitrário $V(x)$, desde que este potencial $V(x)$ seja conhecido.

\subsubsection{Generalização para múltiplas barreiras de potencial}

$\mathrm{Na}$ solução de uma estrutura com $n$ barreiras de potencial com e sem polarização aplicada, necessitamos do Método da Matriz Transferência (MMT), e como já discutido na Seção (3.2.1), é necessário conhecer apenas o potencial $V(x)$. Com isso nas próximas seções discutimos a construção do potencial $V(x)$ para os casos com e sem polarização externa aplicada. 


\section{Múltiplas barreiras de potencial com polarização aplicada}

Na solução de uma estrutura com $n$ barreiras de potencial com polarização aplicada, necessitamos do MMT, e como já discutido na Seção (3.2.1), é necessário conhecer o potencial $V(x)$, para as regiões de interesse do problema, ver Fig.(3.7).

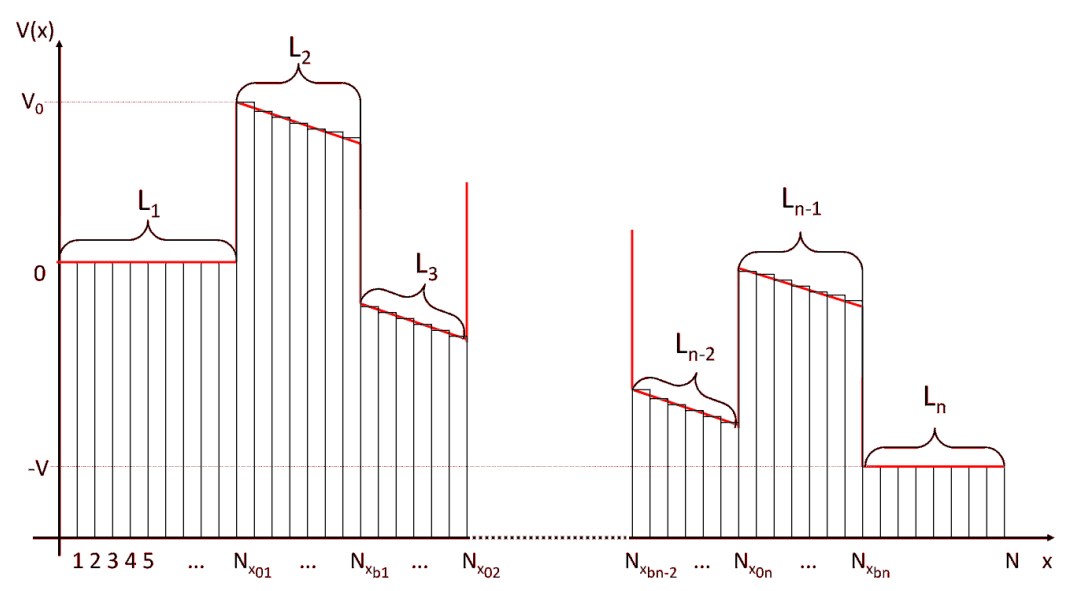

Figura 3.7: Representação de $n$-barreira de potencial com polarização aplicada.

De acordo com a Fig.(3.7), pode-se construir o perfil do potencial $V(x)$ para todas as regiões da estrutura de $n$ barreiras, baseada na Eq.(3.103):

$$
V(x)=\left\{\begin{array}{cc}
0 & 0<x \leqslant N_{x_{01}} \\
V_{0}-\frac{V}{L_{2}}\left(x-N_{x_{01}}\right) & N_{x_{01}}<x \leqslant N_{x_{b 1}} \\
-\frac{V}{L_{3}}\left(x-N_{x_{b 1}}\right) & N_{x_{b 1}}<x \leqslant N_{x_{02}} \\
\cdots & \cdots \\
-\frac{V}{L_{n-2}}\left(x-N_{x_{b n-2}}\right) & N_{x_{b n-2}}<x \leqslant N_{x_{0 n}} \\
V_{0}-\frac{V}{L_{n-1}}\left(x-N_{x_{0 n}}\right) & N_{x_{0 n}}<x \leqslant N_{x_{b n}} \\
-V & N_{x_{b n}}<x \leqslant N
\end{array}\right.
$$

De posse da Eq.(3.104) e da Eq.(3.96) que representam o Método da Matriz Transfe rência utilizando a aproximação do método dos retângulos (AMR), pode-se aplicar este resultado para qualquer número de barreira com polarização aplicada, na próxima Seção iremos analisar a mesma generalização só que agora aplicada as múltiplas barreiras de potencial sem polarização aplicada, para demonstrar a versatilidade do método da matriz transferência apresentado anteriormente. 
Múltiplas barreiras de potencial sem polarização aplicada

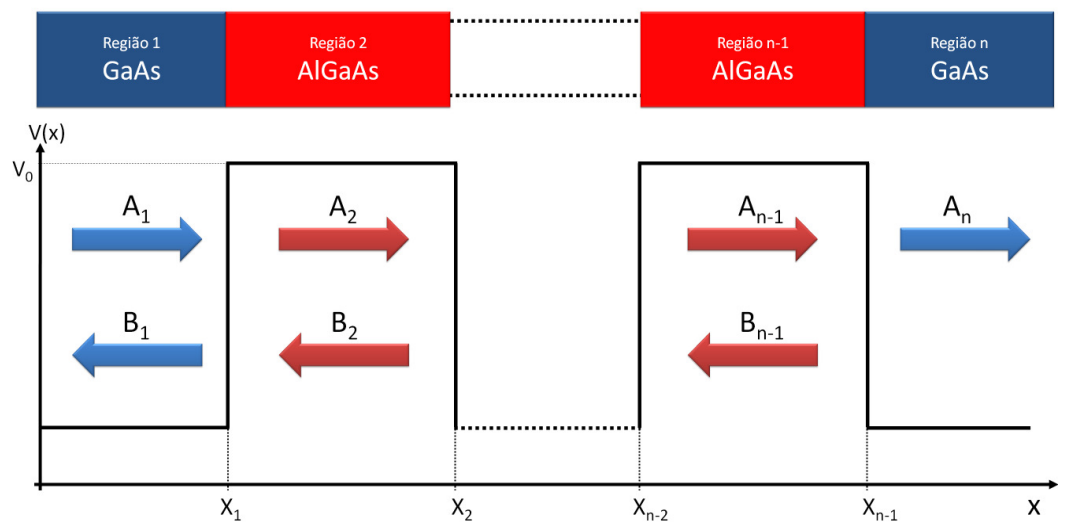

Figura 3.8: Representação de $n$-barreira de potencial sem polarização aplicada.

Esta seção trata essencialmente de validar o MMT empregado em uma estrutura com $n$ barreiras de potencial sem polarização aplicada, como esquematizado na Fig.(3.8), a partir de uma generalização dos resultados obtidos previamente nas Seção (3.2.2). Para isso, tem-se apenas que conhecer o perfil de potencial $V(x)$ sem a polarização externa aplicada,

$$
V(x)=\left\{\begin{array}{cc}
0 & 0<x \leqslant x_{1} \\
V_{0} & x_{1}<x \leqslant x_{2} \\
\cdots & \\
V_{0} & x_{n-2}<x \leqslant x_{n-1} \\
0 & x>x_{n-1}
\end{array}\right.
$$

De posse da Eq.(3.105) e da Eq.(3.99) que representam o Método da Matriz Transfe rência utilizando a aproximação do método dos retângulos (AMR), pode-se aplicar este resultado para qualquer número de barreira sem polarização aplicada.

\subsubsection{Resultados de múltiplas barreiras com polarização externa aplicada}

Utilizando a generalização do MMT para múltiplas barreiras de potencial com polarização externa aplicada, mostrada na Eq.(3.96), pode-se obter o coeficiente de transmissão T, Eq.(3.99), para uma, duas, três e quatro barreiras de potencial com polarização externa aplicada. 


\section{Uma barreira de potencial}

A Eq.(3.99) é utilizada para calcular o coeficiente de transmissão de uma partícula através de uma barreira de potencial unidimensional com polarização aplicada. Com este resultado foi calculado o logarítmo do coeficiente de transmissão em função da tensão aplicada, $V$, para um valor fixo de energia, $E=0,05 \mathrm{eV}$, como realizado por Dresselhaus na Ref. [54]. O resultado obtido para uma barreira com altura $V_{0}=0,2 \mathrm{eV}$, largura da barreira de $100 \AA \mathrm{e}$ $m_{1}^{*}=m_{2}^{*}=0,2 m_{0}$, [54], é mostrada na Fig.(3.9), e está de acordo com a Ref. [54] como pode ser visto na mesma figura.

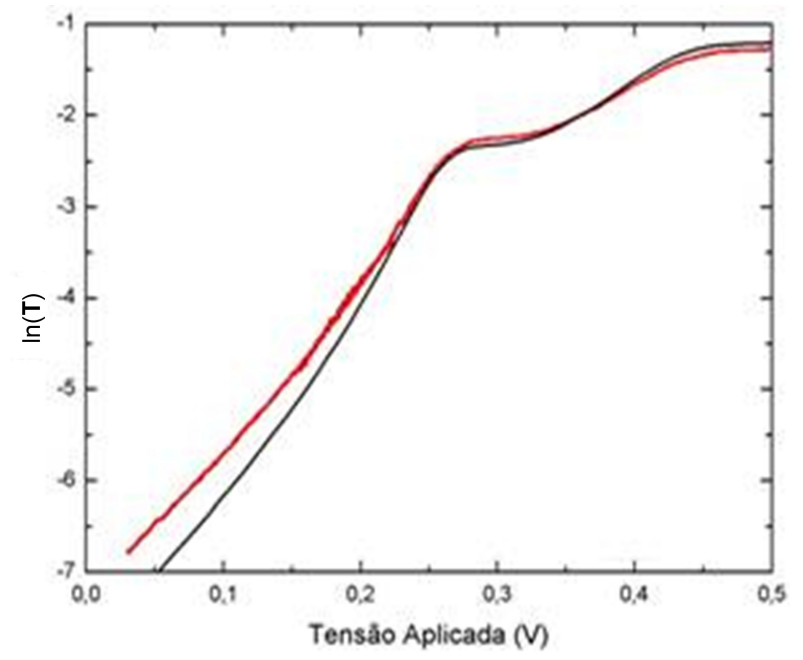

Figura 3.9: Logaritmo do coeficiente de transmissão em função da tensão aplicada de uma barreira de potencial unidimensional. Na figura a linha preta, representa o resultado obtido neste trabalho para uma única barreira com polarização aplicada e a linha vermelha representa a Ref. [54].

\section{Dupla barreira de potencial}

A metodologia usada por Levi [49] pode agora ser utilizada para se calcular o coeficiente de transmissão, T, para uma heteroestrutura com duas barreiras de potencial. No entanto, o método, como apresentado na Seção (3.2.1), requer o conhecimento do perfil de potencial $V(x)$.

A construção do potencial $V(x)$, para duas barreiras, inclui agora um poço de potencial, como pode ser visto na Fig.(3.10), a qual se encontra dividida em N retângulos. 


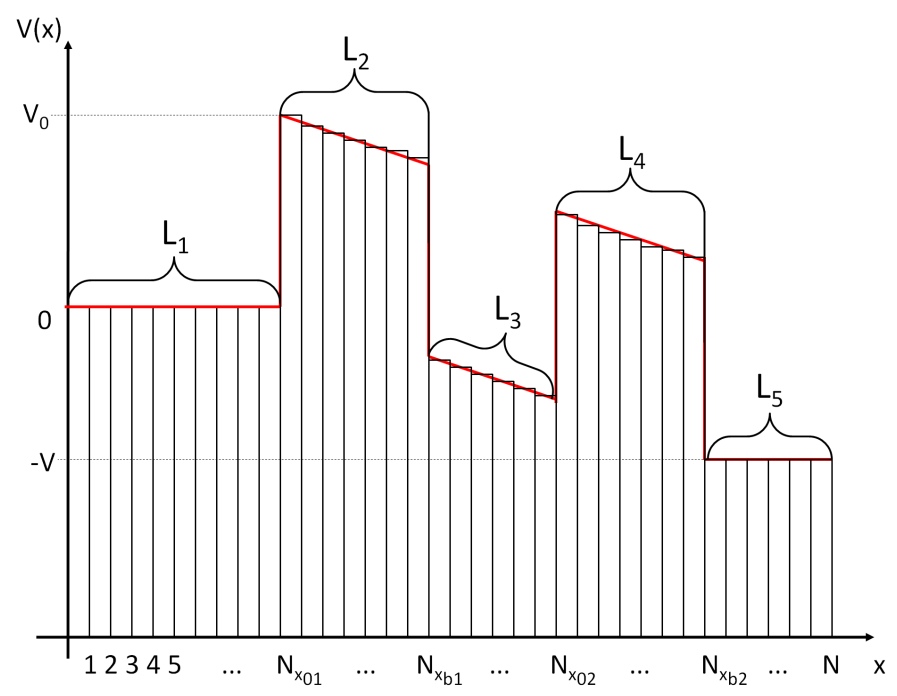

Figura 3.10: Representação de uma dupla bareira de potencial com polarização aplicada.

Resumindo:

$$
V(x)=\left\{\begin{array}{cc}
0 & 0<x \leqslant N_{x_{01}} \\
V_{0}-\frac{V}{L_{2}}\left(x-N_{x_{01}}\right) & N_{x_{01}}<x \leqslant N_{x_{b 1}} \\
-\frac{V}{L_{3}}\left(x-N_{x_{b 1}}\right) & N_{x_{b 1}}<x \leqslant N_{x_{02}} \\
V_{0}-\frac{V}{L_{4}}\left(x-N_{x_{02}}\right) & N_{x_{02}}<x \leqslant N_{x_{b 2}} \\
-V & N_{x_{b 2}}<x \leqslant N
\end{array}\right.
$$

onde $L_{2}=\left(N_{x_{b 1}}-N_{x_{01}}\right)$ e $L_{4}=\left(N_{b_{2}}-N_{x_{02}}\right)$ representam as larguras das barreiras e $L_{3}=$ $\left(N_{x_{02}}-N_{x_{b 1}}\right)$ a largura do poço de potencial.

A Eq.(3.99) é utilizada para calcular o coeficiente de transmissão de uma partícula através de uma barreira de potencial unidimensional com polarização aplicada. Sabendo que as barreiras tem comprimento de $20 \AA$ e o poço de $50 \AA$, cujas massas efetivas em suas regiões são $0,067 m_{0}$ e $0,1087 m_{0}$, Ref. [55], respectivamente, e considerando a altura das barreiras, $V_{0}=0,5 \mathrm{eV}$, pode-se encontrar o logaritmo do coeficiente de transmissão para uma tensão aplicada, $\mathrm{V}$, como representado na Ref. [55] para $0,16 \mathrm{~V}$ e $0,4 \mathrm{~V}$. Os resultados para diferentes tensões foram constrastadas nas Figs.(3.11-a) e (3.11-b) que representam as tensões aplicadas, 0,16 e 0, $4 V$, respectivamente, de acordo com a Ref. [55]. Os resultados mostrados na Fig.(3.11) revelam pleno acordo dos resultados obtido a partir da Eq.(3.99) com aqueles encontrados por Vatannia [55]. 

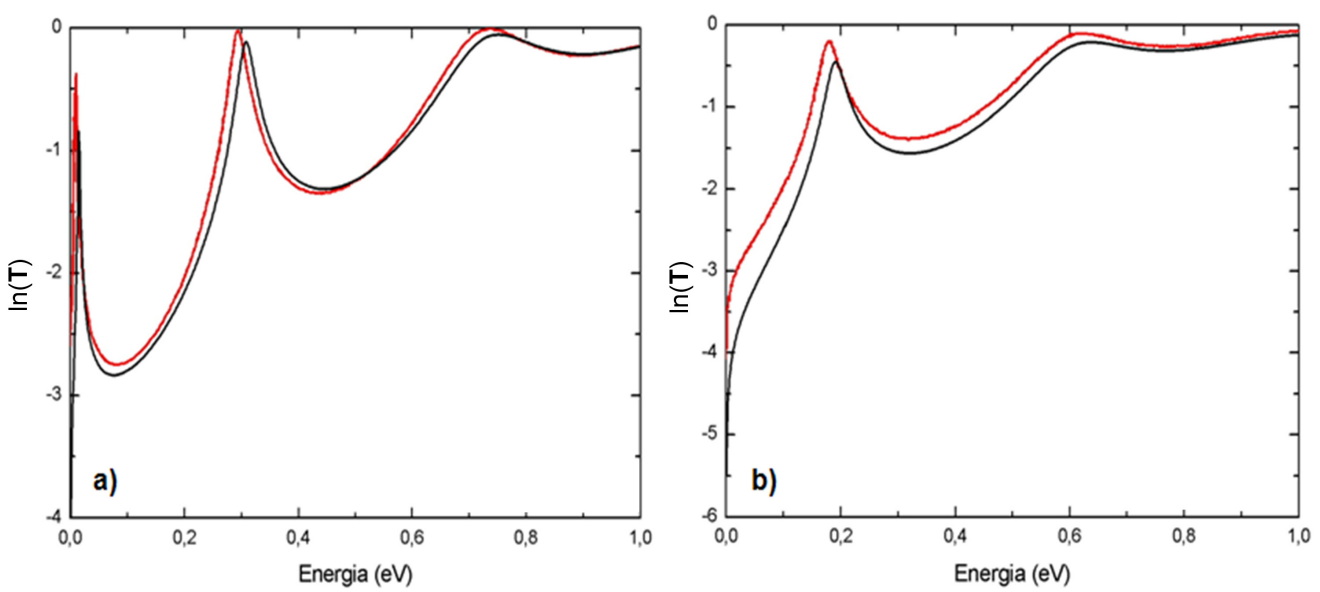

Figura 3.11: Coeficiente de transmissão em função da energia de uma dupla barreira: a) Tensão aplicada: 0,16V. b) Tensão aplicada: 0,4V. Em ambas as figuras: a linha preta, representa o nosso resultado para uma dupla barreira com polarização aplicada e a linha vermelha representa a Ref. [55].

\section{Tripla barreira de potencial}

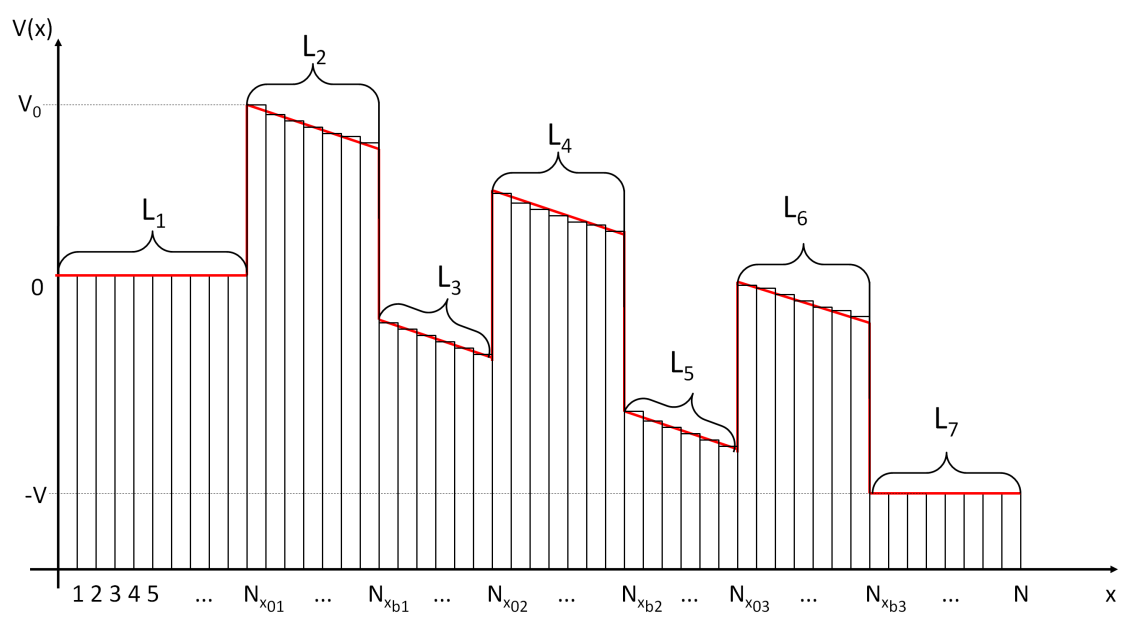

Figura 3.12: Representação de uma tripla barreira de potencial com polarização aplicada.

Na solução de uma estrutura com três barreiras de potencial com polarização aplicada, necessitamos do MMT, e como já discutido na Seção (3.2.3), é necessário conhecer também o potencial $V(x)$, para as regiões de interesse para o problema, ver Fig.(3.12).

Analogamente, na Seção (3.2.3), pode-se escrever o potencial $V(x)$ para todas as 
regiões, de acordo com a Fig.(3.12), de forma resumida:

$$
V(x)=\left\{\begin{array}{cc}
0 & 0<x \leqslant N_{x_{01}} \\
V_{0}-\frac{V}{L_{2}}\left(x-N_{x_{01}}\right) & N_{x_{01}}<x \leqslant N_{x_{b 1}} \\
-\frac{V}{L_{3}}\left(x-N_{x_{01}}\right) & N_{x_{b 1}}<x \leqslant N_{x_{02}} \\
V_{0}-\frac{V}{L_{4}}\left(x-N_{x_{02}}\right) & N_{x_{02}}<x \leqslant N_{x_{b 2}} \\
-\frac{V}{L_{5}}\left(x-N_{x_{b 2}}\right) & N_{x_{b 2}}<x \leqslant N_{x_{03}} \\
V_{0}-\frac{V}{L_{6}}\left(x-N_{x_{03}}\right) & N_{x_{03}}<x \leqslant N_{x_{b 3}} \\
-V & N_{x_{b 3}}<x \leqslant N
\end{array}\right.
$$

onde $L_{2}=\left(N_{x_{b 1}}-N_{x_{01}}\right), L_{4}=\left(N_{b_{2}}-N_{x_{02}}\right)$ e $L_{6}=\left(N_{x_{b 3}}-N_{x_{03}}\right)$ representam as larguras das barreiras e $L_{3}=\left(N_{x_{02}}-N_{x_{b 1}}\right), L_{5}=\left(N_{x_{03}}-N_{x_{b 2}}\right)$ representam as larguras do poço de potencial.
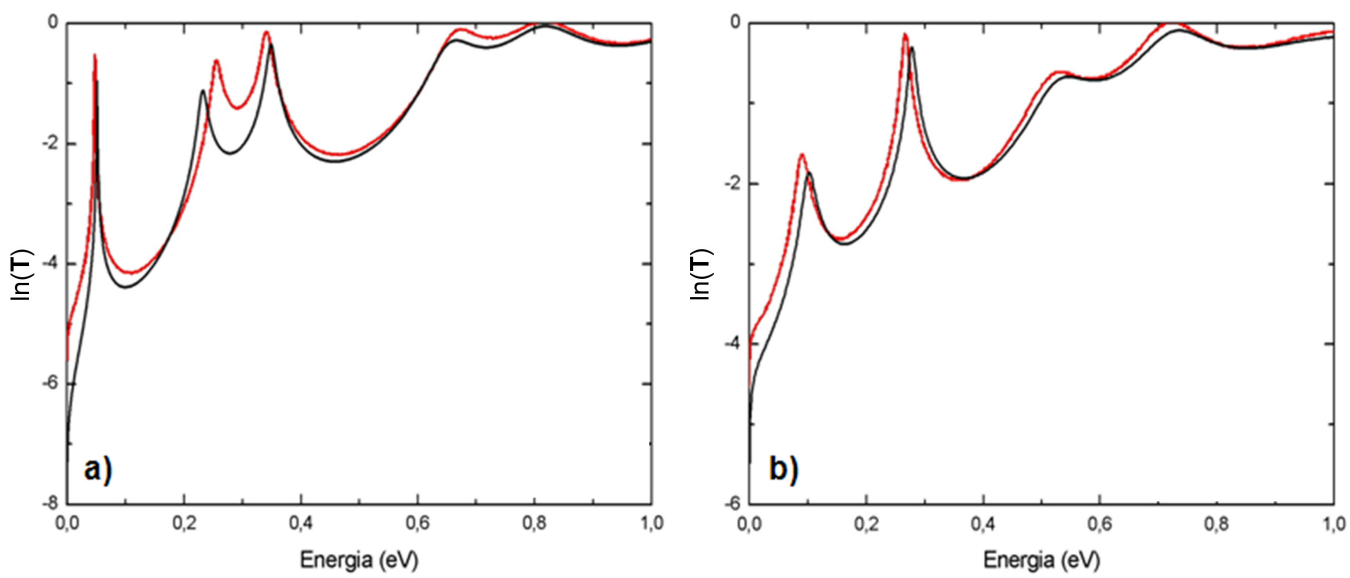

Figura 3.13: Logaritmo do coeficiente de transmissão em função da energia de uma tripla barreira: a) Tensão aplicada de: 0,16V. b) Tensão aplicada de: 0,4V. Em ambas as figuras: a linha preta, representa o nosso resultado para uma tripla barreira com polarização aplicada e a linha vermelha representa a Ref. [55].

A Eq.(3.99) é utilizada para calcular o coeficiente de transmissão de uma partícula através de uma tripla barreira de potencial unidimensional com polarização aplicada. Sabendo que as barreiras tem comprimento de $20 \AA$ e o poço de $50 \AA$, cujas massas efetivas em suas regiões são $0,067 m_{0}$ e $0,1087 m_{0}$, Ref. [55], respectivamente, considerando a altura das barreiras, $V_{0}=0,5 \mathrm{eV}$. Pode-se encontrar o logaritmo do coeficiente de transmissão para uma tensão aplicada, V, como representada na Ref. [55] para $0,16 \mathrm{~V}$ e $0,4 \mathrm{~V}$. Os resultados para diferentes tensões foram constrastadas nas Figs.(3.13-a) e (3.13-b) que representam as tensões aplicadas, 0,16 e $0,4 V$, respectivamente, de acordo com a Ref. [55]. Os resultados mostrados na Fig.(3.13) 
revelam pleno acordo dos resultados obtido a partir da Eq.(3.99) com aqueles encontrados por Vatannia [55].

\section{Quádrupla barreira de potencial}

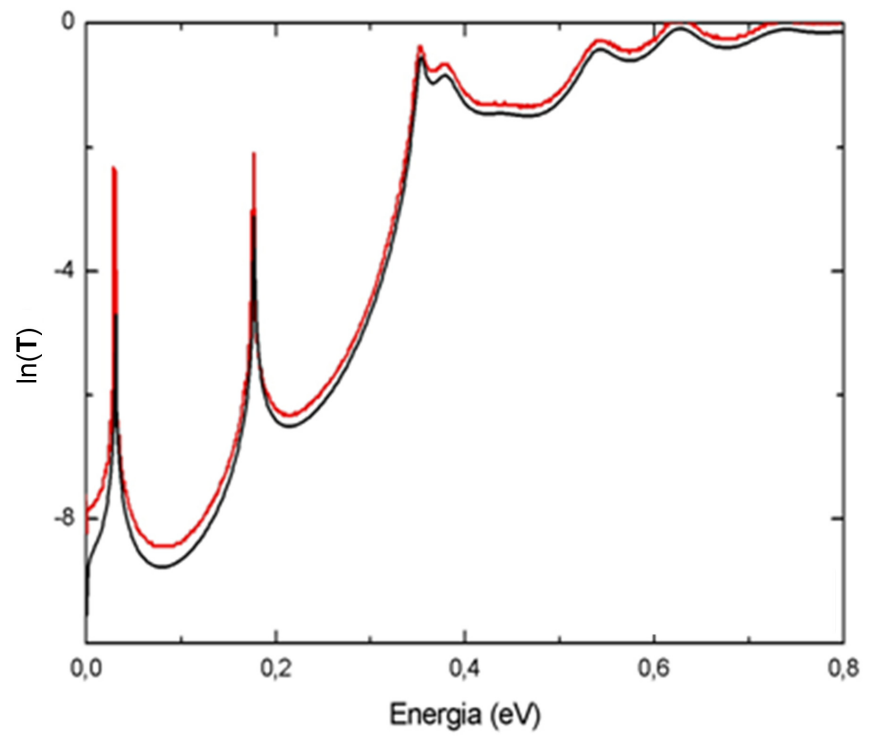

Figura 3.14: Coeficiente de transmissão em função da energia de uma quadrúpla barreira, para uma tensão aplicada de $0,3 \mathrm{~V}$. A linha preta representa o resultado deste trabalho para o caso da quadrupla barreira de potencial com polarização aplicada e a linha vermelha representa o resultado da Ref. [55].

Para aferir o resultado generalizado para n-barreiras, pode-se escolher $n=4$, com o comprimentos das barreiras $35 \AA$ e os poços medindo: 110,28, 2 e $22 \AA$ respectivamente, a altura das barreiras de $V_{0}=0,5 \mathrm{eV}$ e as massas efetivas nas regiões das barreiras e poços sendo, $0,067 m_{0}$ e $0,1043 m_{0}$, Ref. [55], respectivamente e a tensão aplicada de $V=0,3 V$. Ajustando o potencial (3.104) para $n=4$ barreiras e a Eq.(3.99), tem-se o coeficiente de transmissão em função da energia, onde os resultados foram contrastados com os resultados encontrados na Ref. [55], como pode-se verificar na Fig.(3.14).

\subsubsection{Resultados de múltiplas barreiras sem polarização externa aplicada}

Com a intenção de validar o formalismo do MMT apresentado na Seção (3.2.1) para múltiplas barreiras de potencial sem polarização aplicada, nesta seção calculou-se o coeficiente de transmissão para uma, duas, três e cinco barreiras de potencial. 


\section{Uma barreira de potencial}

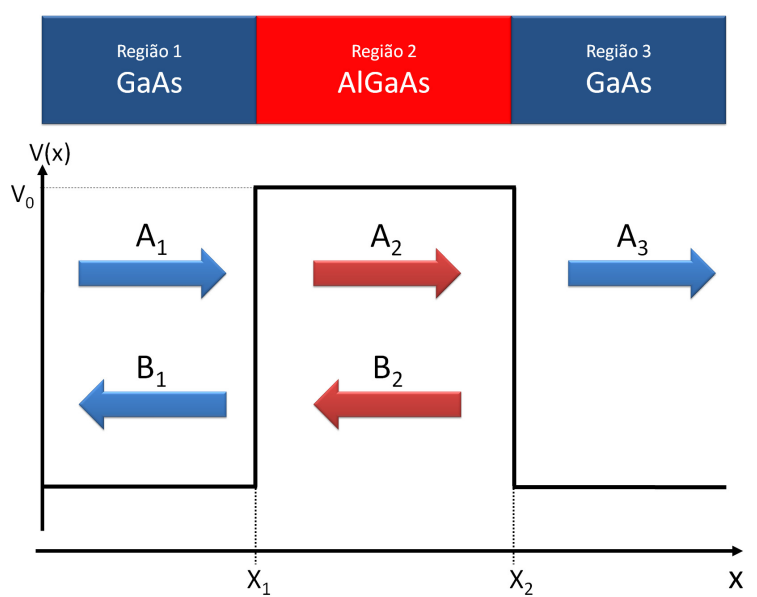

Figura 3.15: Representação da barreira de potencial finito de comprimento $x_{2}-x_{1}$ e altura $V_{0}$.

Considere a sequência de camadas semicondutoras mostrada na Fig.(3.15). Esquematicamente, pode-se representar o diagrama de banda de condução da estrutura como apresentado na Fig.(3.15), com uma barreira de potencial de altura $V_{0}$. De posse do MMT desenvolvido na Seção (3.2.1), é necessário conhecer apenas o perfil de potencial $V(x)$ :

$$
V(x)=\left\{\begin{array}{cc}
0 & 0<x \leqslant x_{1} \\
V_{0} & x_{1}<x \leqslant x_{2} \\
0 & x>x_{2}
\end{array}\right.
$$

De posse do potencial (3.108) e a Eq.(3.99) tem-se o coeficiente de transmissão, T, para uma barreira de potencial sem polarização aplicada. Com isso calculou-se o coeficiente de transmissão para duas estruturas distintas. A primeira estrutura é uma heteroestrutura semicondutora de $G a A s / A l G a A s / G a A s$, cuja representação esquemática encontra-se detalhada na Fig.(3.15), a qual forma uma barreira de potencial, como aquela proposta por Brennan na Ref. [48]. Para esta primeira estrutura, primeiramente, foi assumido a largura da barreira como $20 \AA$ e a altura da barreira, $V_{0}=0,347 \mathrm{eV}$, com as massas sendo iguais a massa do elétron livre, ou seja, $m_{1}^{*}=m_{2}^{*}=m_{3}^{*}=m_{0}$, Ref.[48].

Através do formalismo descrito na Seção (3.2.1) e dos parâmetros indicados acima, calculou-se o coeficiente de transmissão em função da energia da partícula incidente para as duas larguras de barreira, e o resultados é mostrado nas Figs.(3.16) e (3.17). 


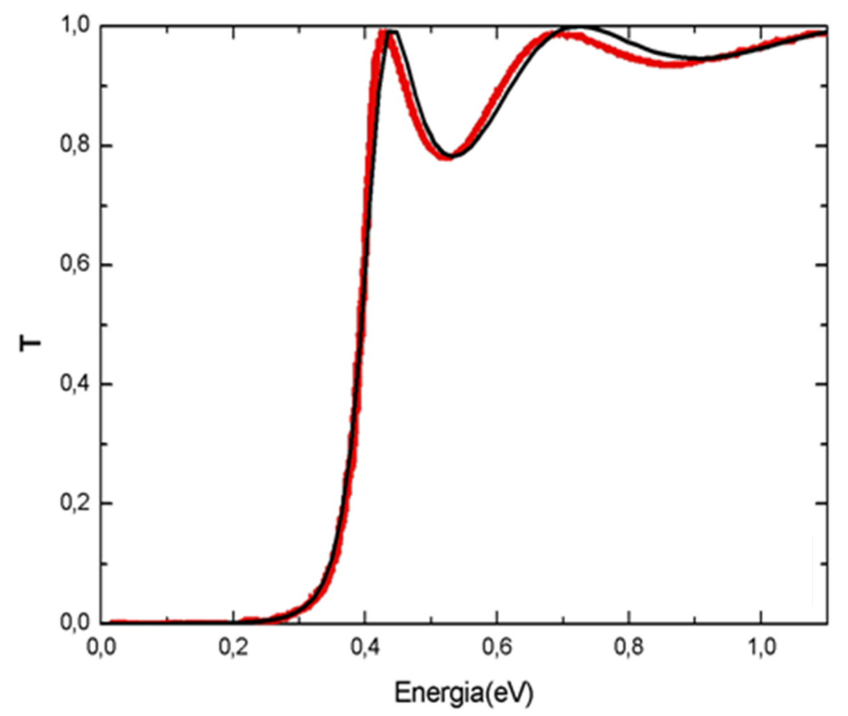

Figura 3.16: Coeficiente de transmissão através de uma barreira de potencial em função da energia da partícula incidente na barreira com o comprimento de $20 \AA$ e potencial de $0,347 \mathrm{eV}$, onde a linha preta representa o resultado do formalismo desenvolvido na Seção (3.2.4) e a linha vermelha representa o trabalho proposto por Brennan na Ref. [48].

Posteriormente, tomou-se a largura da barreira como $10 \AA$ e a altura da barreira, com as massas sendo iguais a massa do elétron livre, ou seja, $m_{1}^{*}=m_{2}^{*}=m_{3}^{*}=m_{0}$, Ref.[48].

De imediato, analisando os resultados obtidos através das Figs.(3.16) e (3.17) verifica-se o efeito de tunelamento nas estruturas investigadas, em que uma partícula com energia menor que a altura da barreira, $V_{0}$, tem uma probabilidade não-nula de passar através da barreira. Adicionalmente, pode-se observar que é necessário uma energia muito maior do que $V_{0}$, para que a partícula incidente não seja mais perturbada pelo potencial da barreira.

Vale observar que, os resultados obtidos para o coeficiente de transmissão em função da energia da partícula incidente mostrado nas Figs.(3.16) e (3.17) estão de acordo com os resultados obtidos por Brennan na Ref. [48], o que evidencia a validade do formalismo apresentado na Seção $(3.2 .1)$.

\section{Dupla barreira de potencial}

Considere a sequência de camadas semicondutoras mostrada na Fig.(3.18). Esquematicamente, pode-se representar o diagrama de banda de condução da estrutura, como apresentado na Fig.(3.18), como uma dupla barreira de potencial de altura $V_{0}$. De posse do MMT desenvolvido na Seção (3.2.1), é necessário conhecer apenas o perfil de potencial $V(x)$ : 


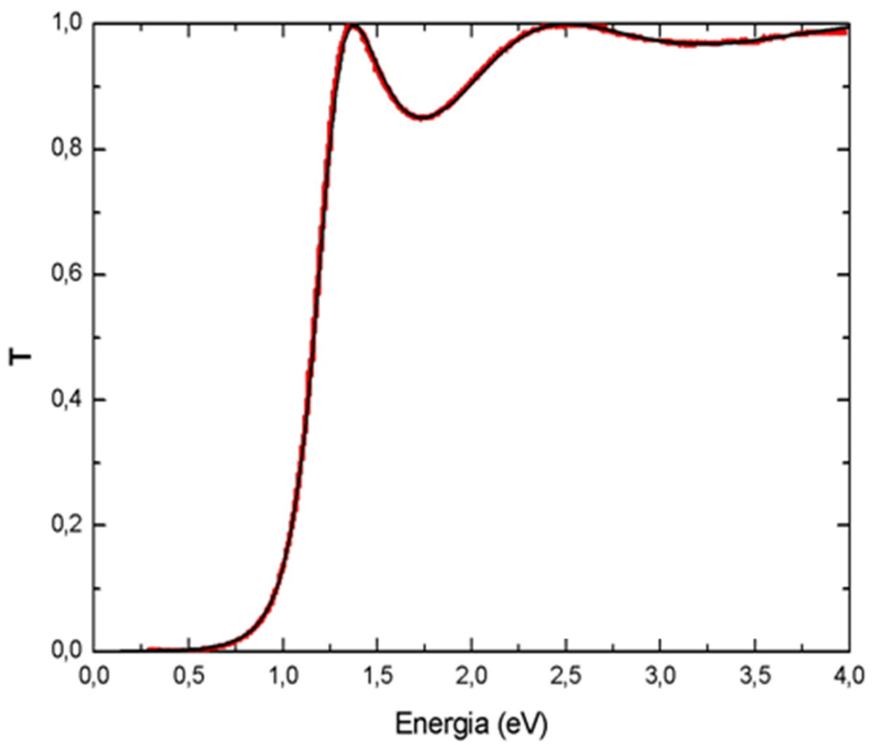

Figura 3.17: Coeficiente de transmissão através de uma barreira de potencial em função da energia da partícula incidente na barreira com o comprimento de $10 \AA$ e potencial de $1,0 \mathrm{eV}$, onde a linha preta representa o resultado do formalismo desenvolvido na Seção (3.2.4) e a linha vermelha representa o trabalho proposto por Brennan na Ref. [48].

$$
V(x)=\left\{\begin{array}{cc}
0 & 0<x \leqslant x_{1} \\
V_{0} & x_{1}<x \leqslant x_{2} \\
0 & x_{2}<x \leqslant x_{3} \\
V_{0} & x_{3}<x \leqslant x_{4} \\
0 & x>x_{4}
\end{array}\right.
$$

Dispondo-se do potencial (3.109) e a Eq.(3.99) tem-se o coeficiente de transmissão, $\mathbf{T}$, para duas barreiras de potencial sem polarização aplicada. Uma tal estrutura foi proposta por Dresselhaus na Ref. [54]. Para a obtenção do coeficiente de transmissão foi utilizado uma estrutura de dupla barreira, no qual a barreira possui um comprimento de $50 \AA$ e o poço de potencial com o comprimento de $60 \AA$ e a altura da barreira de $0,3 \mathrm{eV}$, onde tomou-se $k_{1}=k_{3}=k_{5}$ e $k_{2}=k_{4}, m_{1}^{*}=m_{3}^{*}=m_{5}^{*}=m_{G a A s}^{*}=0,067 m_{0}, m_{2}^{*}=m_{4}^{*}=m_{A l G a A s}^{*}=0,1087 m_{0}$, Ref. [54].

Observando-se a Fig.(3.19) pode-se verificar a existência de dois picos de transmissão, que correspondem a dois auto-estados de energias da partícula no poço de potencial finito presente na estrutura. De imediato, conclui-se que, no caso de estruturas com mais de uma barreira, surge o efeito de tunelamento ressonante, o qual representa uma interferência construtiva devido 


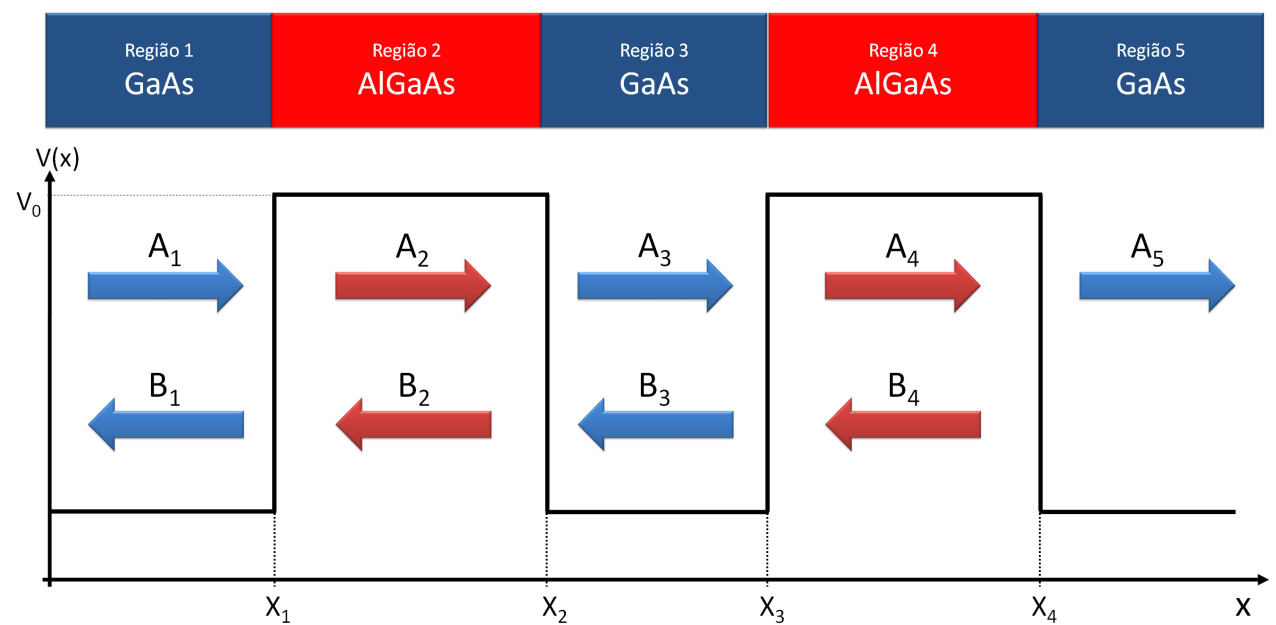

Figura 3.18: Representação do problema da barreira dupla de potencial finito, de altura $V_{0}$.

a presença de poços quânticos entre barreiras, resultando em picos de ressonância nas curvas de transmissividade em função da energia da partícula incidente. Além disso, pode-se observar também, através da Fig.(3.19), que, para energias bem abaixo da altura da barreira, 0, $3 \mathrm{eV}$, existe a probabilidade não-nula da partícula tunelar pela barreira. Vale a pena observar que, os picos que aparecem na Fig.(3.19) correspondem precisamente aos auto-valores de energia do poço quântico.

Como um resultado adicional, calculamos o coeficiente de transmissão sugerido na Ref. [53], para uma estrutura de dupla barreira, sendo o comprimento da barreira de $20 \AA$, do poço de potencial de $50 \AA$ e a altura da barreira de $0,5 \mathrm{eV}$. Adicionalmente, tomou-se $k_{1}=k_{3}=k_{5} \mathrm{e}$ $k_{2}=k_{4}, m_{1}^{*}=m_{3}^{*}=m_{5}^{*}=m_{G a A s}^{*}=0,067 m_{0}, m_{2}^{*}=m_{4}^{*}=m_{A l G a A s}^{*}=0,1087 m_{0}$, Ref. [55].

Vale à pena salientar que os resultados obtidos para o coeficiente de transmissão em função da energia da partícula incidente mostrado nas Figs.(3.19) e (3.20) estão de acordo com os resultados obtidos por Dresselhaus na Ref. [54] e Tsu e Esaki na Ref. [53], o que evidencia a validade da implementação do formalismo apresentado na Seção (3.2.1).

\section{Tripla barreira de potencial}

Considere a sequência de camadas semicondutoras mostrada na Fig.(3.21). Esquematicamente, pode-se representar o diagrama de banda de condução da estrutura como apresentado na Fig.(3.21), com uma tripla barreira de potencial de altura $V_{0}$. De posse do MMT desenvolvido 


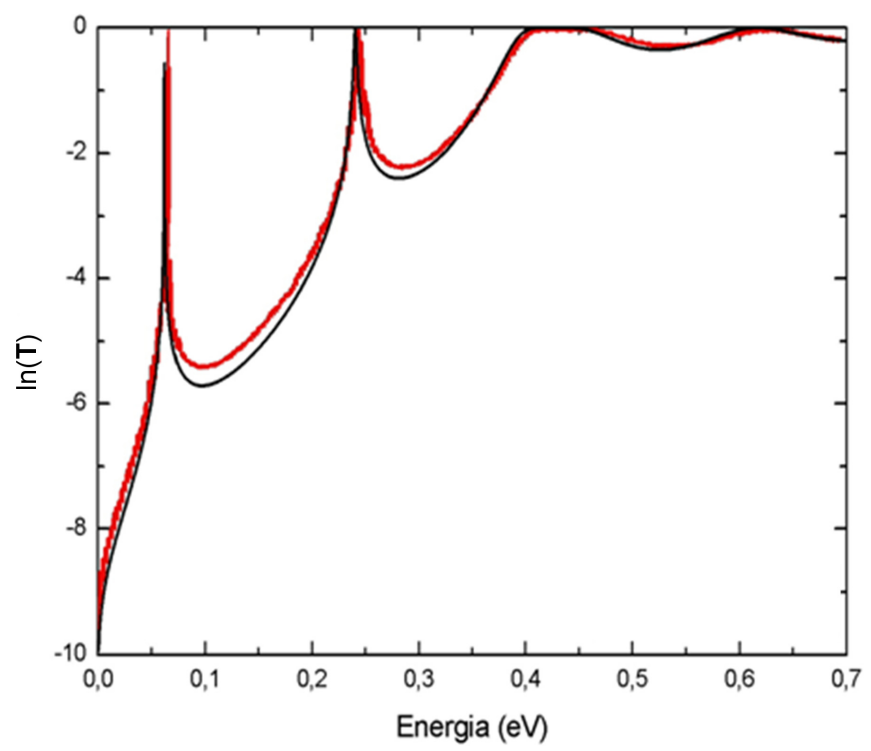

Figura 3.19: Coeficiente de transmissão através de uma dupla barreira de potencial em função da energia da partícula incidente. As barreiras apresentam comprimento de $50 \AA$ e altura 0,3 $\mathrm{eV}$, e o poço quântico um comprimento de $60 \AA$. A linha em preto representa o resultado obtido através do formalismo desenvolvido na Seção (3.2.4) e a linha em vermelho representa o resultado obtido por Dresselhaus na Ref. [54].

na Seção (3.2.1), é necessário conhecer apenas o perfil de potencial $V(x)$ :

$$
V(x)=\left\{\begin{array}{cc}
0 & 0<x \leqslant x_{1} \\
V_{0} & x_{1}<x \leqslant x_{2} \\
0 & x_{2}<x \leqslant x_{3} \\
V_{0} & x_{3}<x \leqslant x_{4} \\
0 & x_{4}<x \leqslant x_{5} \\
V_{0} & x_{5}<x \leqslant x_{6} \\
0 & x>x_{6}
\end{array}\right.
$$

De posse do potencial (3.110) e a Eq.(3.99) tem-se o coeficiente de transmissão, T, para três barreiras de potencial sem polarização aplicada.Com isso, pode-se investigar o tunelamento através da estrutura de tripla barreira proposta por Vatannia et al. na Ref. [55], na qual a barreira de potencial possui um comprimento de $20 \AA$, o poço de potencial um comprimento de $50 \AA$ e a altura da barreira de $0,5 \mathrm{eV}$. Além disso, onde tomou-se $k_{1}=k_{3}=k_{5}=k_{7}$ e $k_{2}=k_{4}=$ $k_{6}, m_{1}^{*}=m_{3}^{*}=m_{5}^{*}=m_{7}^{*}=m_{G a A s}^{*}=0,067 m_{0}, m_{2}^{*}=m_{4}^{*}=m_{6}^{*}=m_{A l G a A s}^{*}=0,1087 m_{0}$, Ref. [55]. O coeficiente de transmissão, $\mathbf{T}$, foi obtido e se encontra em pleno acordo com o resultado 


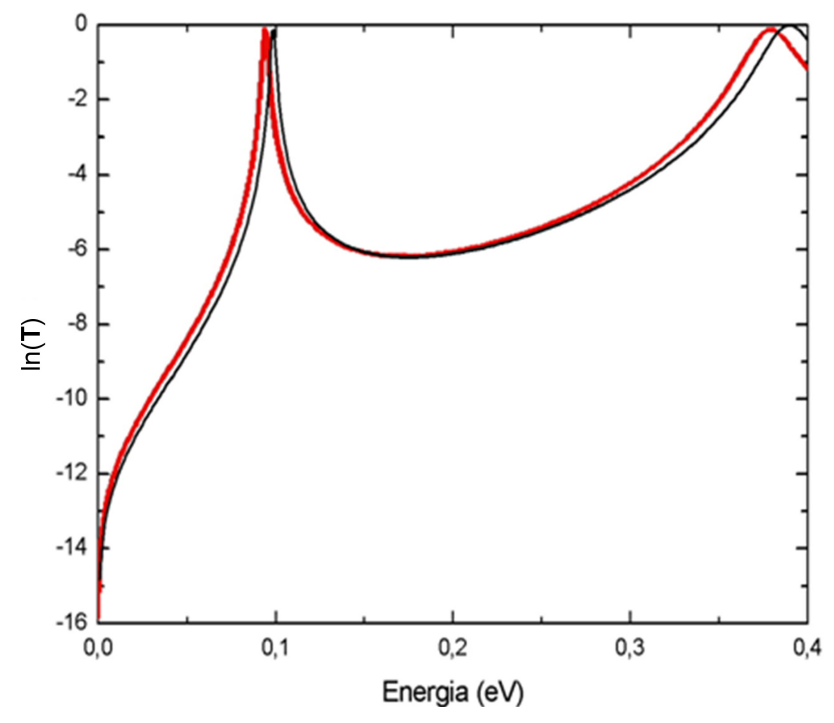

Figura 3.20: Coeficiente de transmissão através de uma dupla barreira de potencial em função da energia da partícula incidente. As barreiras apresentam comprimento de $20 \AA$ e altura 0,5 $\mathrm{eV}$, e o poço quântico um comprimento de $50 \AA$. A linha em preto representa o resultado obtido através do formalismo desenvolvido na Seção (3.2.4) e a linha em vermelho representa o resultado obtido por Tsu e Esaki na Ref. [53].

encontrado na Ref. [55], como pode ser facilmente verificado analisando-se a Fig.(3.22).

Como um resultado adicional, calculamos o coeficiente de transmissão sugerido por Tsu e Esaki na Ref. [53], que pode ser visualizada na Fig.(3.23), para uma estrutura de tripla barreira, na qual a barreira possui um comprimento de $20 \AA$, o poço quântico um comprimento de $50 \AA$ e a altura da barreira de $0,5 \mathrm{eV}$. Nestas condições tomou-se $k_{1}=k_{3}=k_{5}=k_{7}$ e $k_{2}=k_{4}=k_{6}$, $m_{1}^{*}=m_{3}^{*}=m_{5}^{*}=m_{7}^{*}=m_{G a A s}^{*}=0,067 m_{0}, m_{2}^{*}=m_{4}^{*}=m_{6}^{*}=m_{A l G a A s}^{*}=0,1087 m_{0}$, Ref. [55].

\section{Quíntupla barreira de potencial}

Para finalizar, através do resultado obtido na Eq.(3.99) e do perfil de potencial $V(x)$ análogo a Eq.(3.104), foi analisada uma heteroestrutura semicondutora que se assemelha com àquela representada esquematicamente na Fig.(3.8), fazendo-se $n=5$, a qual deve ser investigada usando-se o formalismo desenvolvido na Seção (3.2.1). Uma tal estrutura foi proposta por Tsu e Esaki na Ref. [53]. Para a obtenção do coeficiente de transmissão aqui, foi utilizado uma estrutura de cinco barreiras, no qual a barreira possui um comprimento de $20 \AA$ e o poço de potencial com o comprimento de $50 \AA$ e a altura da barreira de $0,5 \mathrm{eV}$, onde tomou-se $k_{1}=k_{3}=k_{5}=k_{7}=k_{9}=k_{11} \mathrm{e} k_{2}=k_{4}=k_{6}=k_{8}=k_{10}, m_{1}^{*}=m_{3}^{*}=m_{5}^{*}=m_{7}^{*}=m_{9}^{*}=m_{11}^{*}=$ 


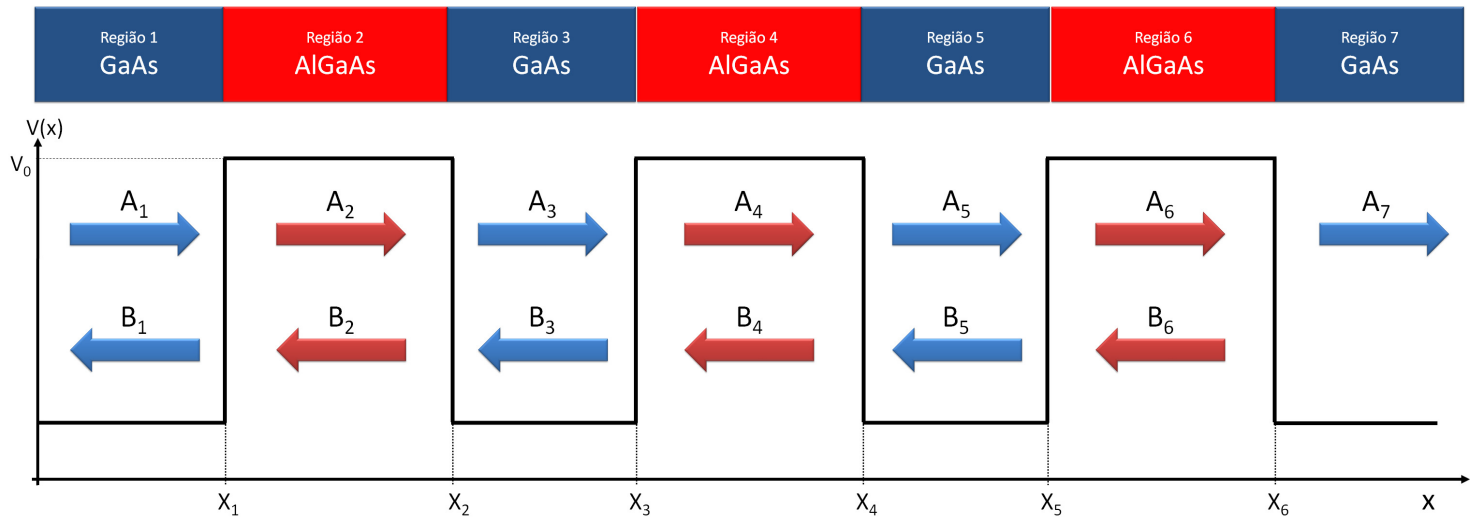

Figura 3.21: Representação do problema da barreira tripla de potencial finito de altura $V_{0}$.

$m_{G a A s}^{*}=0,067 m_{0}, m_{2}^{*}=m_{4}^{*}=m_{6}^{*}=m_{8}^{*}=m_{10}^{*}=m_{A l G a A s}^{*}=0,1087 m_{0}$, Ref. [55].

Tendo estudado o desenvolvimento e a validação do Método da Matriz Transferência com o objetivo de obter o coeficiente de transmissão $\mathbf{T}(E, V)$ para múltiplas barreiras de potencial com e sem polarização, agora pode-se calcular a densidade de corrente, conforme desenvolvido na Seção (3.1).

\subsection{Cálculo da densidade de corrente para heteroestruturas de duas e três barreiras}

As duas primeiras estruturas a serem investigadas neste trabalho para as quais serão realizados os cálculos de densidade de corrente usando-se a Eq.(3.17) e o formalismo de MMT desenvolvido nas seções anteriores, são as estruturas mostrada nas Fig.(3.25), que foram propostos por Tsu e Esaki [53] num trabalho pioneiro sobre o cálculo de corrente de tunelamento em estruturas do tipo superrede, no qual se baseiam inúmeros outros trabalhos encontrados na literatura, apresentando mais de 1352 (em 29 de maio de 2010) citações indexadas pelo banco de dados ISI. Um destes trabalhos foi desenvolvido por Forrest e Meijer [56], que produziram os resultados de corrente de tunelamento contra os quais confrontamos os resultados obtidos nesta dissertação. Os parâmetros utilizados nos cálculos na Ref. [53] são mostrados na Tabela (3.1).

De imediato, a partir das Eqs.(3.17) e (3.99), e conhecendo-se os parâmetros mostrados na Tabela (3.1), pode-se calcular a densidade de corrente de tunelamento como função da tensão aplicada, para os sistemas de heteroestruturas semicondutora $\mathrm{GaAs} / \mathrm{Al}_{0,5} \mathrm{Ga}_{0,5} \mathrm{As}$ mostradas na 


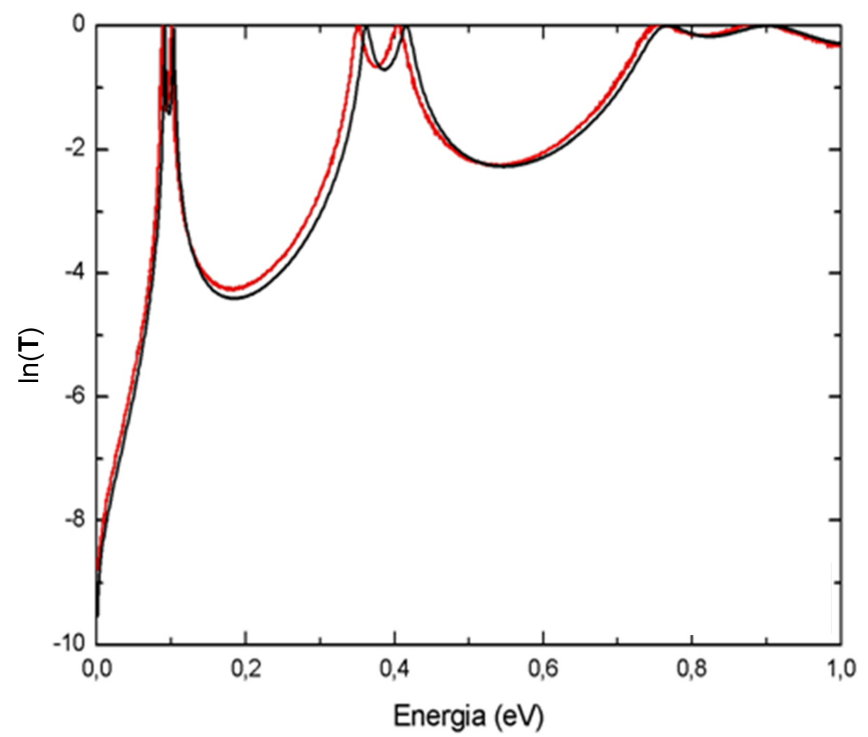

Figura 3.22: Coeficiente de transmissão através de uma tripla barreira de potencial em função da energia da partícula incidente. As barreiras apresentam comprimento de $20 \AA$ e altura 0,5 $\mathrm{eV}$, e o poço quântico um comprimento de $50 \AA$. A linha em preto representa o resultado obtido através do formalismo desenvolvido na Seção (3.2.4) e a linha em vermelho representa o resultado obtido por Vatannia na Ref. [55].

Fig.(3.25). A energia incidente considerada é de $0,005 \mathrm{eV}$, que corresponde ao valor da energia do nível de Fermi no GaAs dopado com uma concentração de elétrons de $n=10^{17} \mathrm{~cm}^{-3}$. O intervalo de tensões aplicadas encontra-se entre zero e $2 \mathrm{~V}$, que foi escolhido por representar um intervalo de tensões capaz de evidenciar como as ressonâncias nestas heteroestruturas influencia a característica densidade de corrente-tensão (J-V).

Os resultados obtidos por Tsu e Esaki [53] serão comparados com aqueles obtidos pelo formalismo descrito nas seções (3.1) e (3.2.1). As Figs.(3.26) e (3.27) mostram as curvas $\ln (\mathrm{J})$ versus $\mathrm{V}$ para heteroestruturas semicondutoras de duas e três barreiras, respectivamente. A curva para a heteroestrutura de duas barreiras apresentada na Fig.(3.26) é particularmente simples devido ao fato de existir apenas um poço em sua estrutura, indicando a existência de um pico de corrente em aproximadamente $0,23 \mathrm{~V}$ e outro próximo a $0,86 \mathrm{~V}$. Ao lado dos picos de corrente vê-se regiões de resistência diferencial negativa, fenômeno que são características das heteroestruturas semicondutoras com barreiras que apresentem o tunelamento ressonante. Com o pico da corrente em $0,23 \mathrm{~V}$ ocorre o alinhamento dos auto-estados do emissor com o autoestado do poço, ocasionando assim o tunelamento ressonante. Com o aumento da tensão ocorre a separação entre os auto-estados do emissor com o poço diminuindo o tunelamento ressonante 


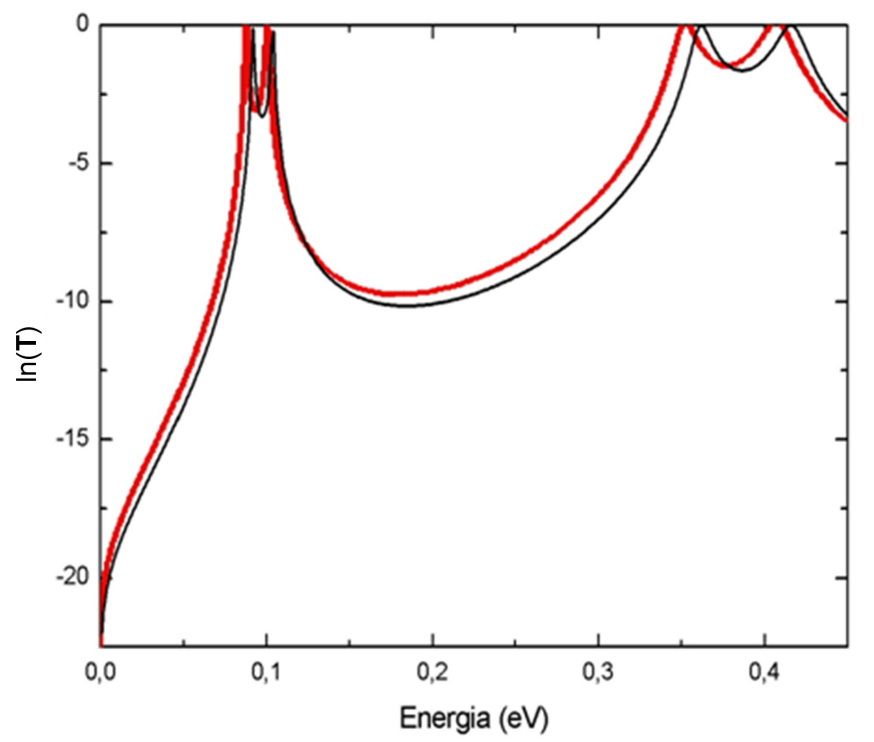

Figura 3.23: Coeficiente de transmissão através de uma tripla barreira de potencial em função da energia da partícula incidente. As barreiras apresentam comprimento de $20 \AA$ e altura 0,5 $\mathrm{eV}$, e o poço quântico um comprimento de $50 \AA$. A linha em preto representa o resultado obtido através do formalismo desenvolvido na Seção (3.2.4) e a linha em vermelho representa o resultado obtido por Tsu e Esaki na Ref. [53].

e com isso aumenta-se a resitência diferencial negativa. Comportamento pode ser análogo para o caso das três barreiras, modificando o número de picos e as regiões de resistência diferencial negativa, como pode ser verificado observando-se a Fig.(3.27).

As curvas obtidas para as heteroestruturas de duas e três barreiras estão mostradas nas Figs.(3.26) e (3.27), as quais encontram-se de acordo com as curvas obtidas por Forrest e Meijer, Ref.[56]. Com os resultados obtidos para a densidade de corrente-tensão das heteroestruturas propostas por Esaki e Tsu [53], consolidado por Forrest e Meijer [56], demonstrou-se a validade

\begin{tabular}{|l|r|}
\hline Altura da barreira & $0,5 \mathrm{eV}$ \\
\hline Largura da barreira & $20 \AA$ \\
\hline Largura do poço & $50 \AA$ \\
\hline Concentração de Al & 0,5 \\
\hline Massa efetiva do elétron na barreira & $0,094^{[56]}$ \\
\hline Massa efetiva do elétron no poço & $0,0636^{[56]}$ \\
\hline Energia incidente & $0,005 \mathrm{eV}$ \\
\hline
\end{tabular}

Tabela 3.1: Parâmetros usados para obtenção da densidade de corrente J para as estruturas mostradas na Fig. (2.34) e (2.35) 


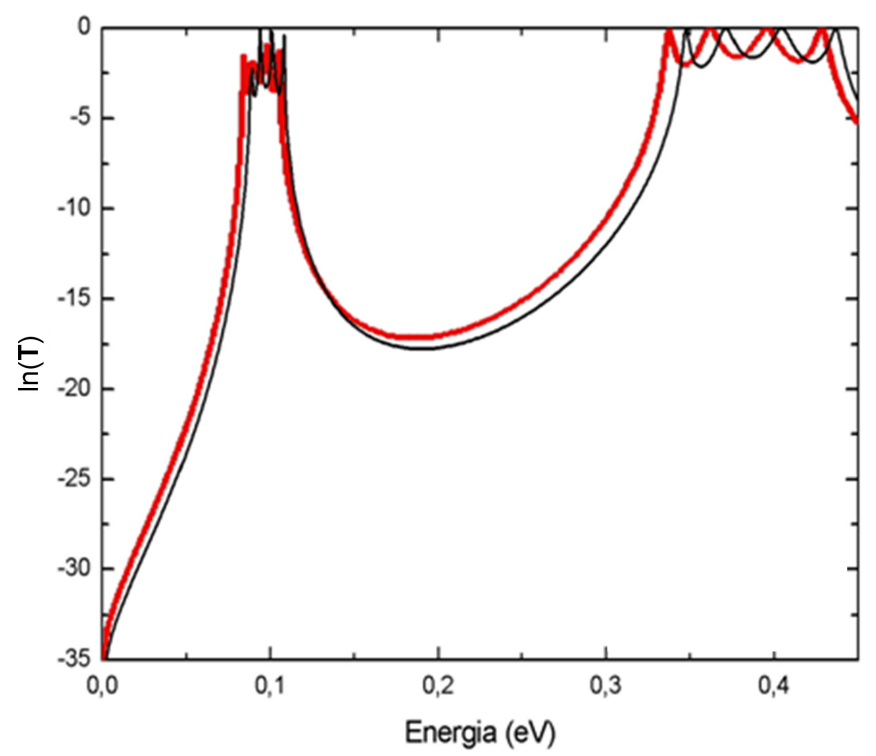

Figura 3.24: Coeficiente de transmissão através de uma quíntupla barreira de potencial em função da energia da partícula incidente. As barreiras apresentam comprimento de $20 \AA$ e altura $0,5 \mathrm{eV}$, e o poço quântico um comprimento de $50 \AA$. A linha em preto representa o resultado obtido através do formalismo desenvolvido na Seção (3.2.4) e a linha em vermelho representa o resultado obtido por Tsu e Esaki na Ref. [53].

do formalismo desenvolvido neste capítulo.

\subsection{Diodo de fio quântico com tunelamento ressonante}

O desenvolvimento das Seções anteriores permite extender estes cálculos analíticos desenvolvidos anteriormente para uma maior compreensão das estruturas de fios quânticos com tunelamento ressonante e propor dispositivos empregando tais estruturas unidimensionais.

Para a modelagem do diodo de tunelamento ressonante de fio quântico, mostrado na Fig.(3.28), tem-se que resolver a eq. de Schrödinger para as coordenadas cilíndricas

$$
\widehat{H} \Psi(\vec{r})=E \Psi(\vec{r})
$$

como o Hamiltoniano

$$
\widehat{H}=-\frac{\hbar^{2}}{2} \vec{\nabla} \frac{1}{\mu(z)} \vec{\nabla}+V(r, \theta, z)
$$

onde $\mu$ é a massa efetiva. Vale salientar que utilizou-se $\mu$ como massa efetiva, pois utilizou-se $m$ como número quântico magnético, que pode ser considerado constante na Região $j$, 


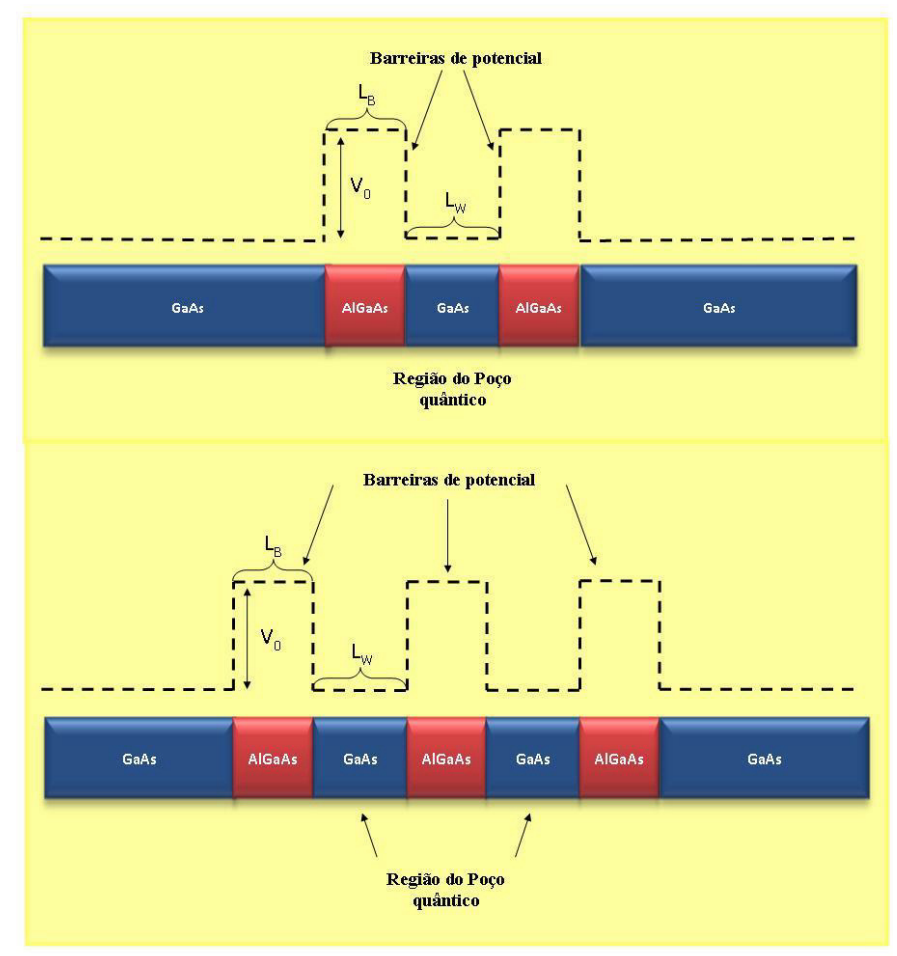

Figura 3.25: Perfil de potencial de uma heteroestutura semicondutora de duas e três barreiras, no qual as barreiras tem $20 \AA$ e os poços $50 \AA$, e a altura da barreira $0,5 \mathrm{eV}$, de acordo com a Ref. [53].

$$
\widehat{H}=-\frac{\hbar^{2}}{2 \mu_{j}} \nabla^{2}+V(r, \theta, z)
$$

que em coordenadas cilíndricas pode ser escrita como

$$
\widehat{H}=-\frac{\hbar^{2}}{2 \mu_{j}}\left[\frac{1}{r} \frac{\partial}{\partial r}\left(r \frac{\partial}{\partial r}\right)+\frac{1}{r^{2}} \frac{\partial^{2}}{\partial \theta^{2}}+\frac{\partial^{2}}{\partial z^{2}}\right]+V(r, \theta, z)
$$

aplicando a Eq.(3.113) na (3.111), tem-se

$$
\left\{-\frac{\hbar^{2}}{2 \mu_{j}}\left[\frac{1}{r} \frac{\partial}{\partial r}\left(r \frac{\partial}{\partial r}\right)+\frac{1}{r^{2}} \frac{\partial^{2}}{\partial \theta^{2}}+\frac{\partial^{2}}{\partial z^{2}}\right]+V(r, \theta, z)\right\} \Psi(r, \theta, z)=E \Psi(r, \theta, z)
$$

rearranjando

$$
\left\{-\frac{\hbar^{2}}{2 \mu_{j}}\left[\frac{1}{r} \frac{\partial}{\partial r}\left(r \frac{\partial}{\partial r}\right)+\frac{1}{r^{2}} \frac{\partial^{2}}{\partial \theta^{2}}+\frac{\partial^{2}}{\partial z^{2}}\right]+V(r, \theta, z)-E\right\} \Psi(r, \theta, z)=0
$$

sabendo que a função de onda $\Psi(r, \theta, z)$ pode ser escrita da seguinte maneira, devido a simetria azimutal de $\theta$ 


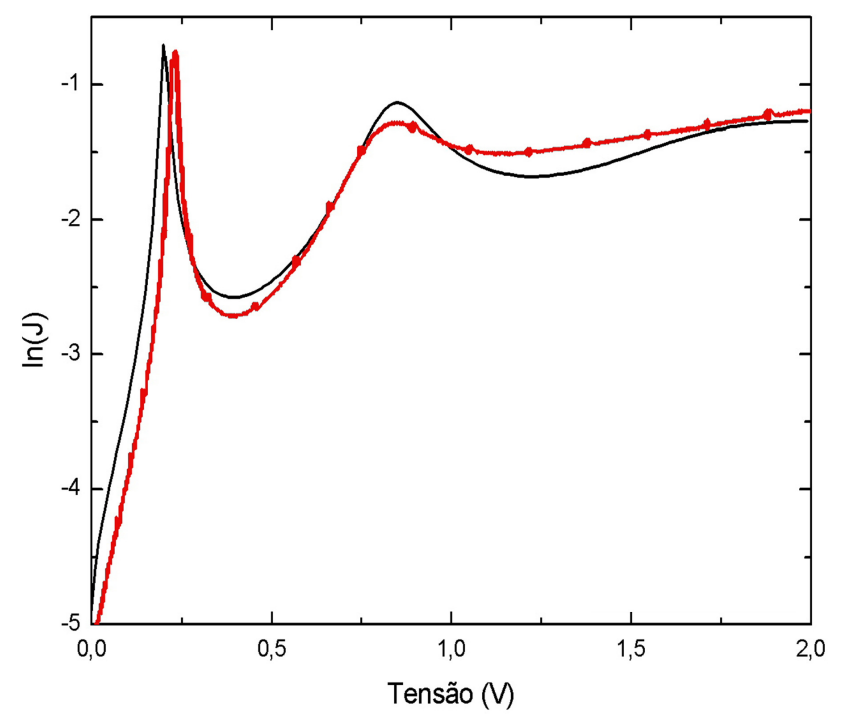

Figura 3.26: Densidade de corrente de tunelamento em função da tensão aplicada para a heteroestrutura semicondutora de duas barreiras, com a largura da barreira de $20 \AA$ e altura 0,5 $\mathrm{eV}$ e a largura do poço de $50 \AA$, no qual considerou-se a energia incidente do elétron com o valor de $0,005 \mathrm{eV}$. A linha preta representa o resultado obtido neste trabalho e a linha vermelha representa o trabalho proposto por Forrest e Meijer na Ref. [56].

$$
\Psi(r, \theta, z)=\frac{1}{\sqrt{2 \pi}} e^{i m \theta} R(r) \varphi(z)
$$

onde $m=0, \pm 1, \pm 2, \ldots$ é o número quântico magnético.

Substituindo a função de onda da Eq.(3.115) na (3.114), tem-se

$$
\left\{-\frac{\hbar^{2}}{2 \mu}\left[\frac{1}{r} \frac{\partial}{\partial r}\left(r \frac{\partial}{\partial r}\right)+\frac{1}{r^{2}} \frac{\partial^{2}}{\partial \theta^{2}}+\frac{\partial^{2}}{\partial z^{2}}\right]+V(r, \theta, z)-E\right\} \frac{1}{\sqrt{2 \pi}} e^{i m \theta} R(r) \varphi(z)=0
$$

após algumas manipulações algébricas, tem-se

$$
\left\{-\frac{\hbar^{2}}{2 \mu}\left[\frac{1}{R(r)}\left[\frac{1}{r} \frac{d R(r)}{d r}+\frac{r}{r} \frac{d^{2} R(r)}{d r^{2}}\right]-\frac{m^{2}}{r^{2}}+\frac{1}{\varphi(z)} \frac{d^{2} \varphi(z)}{d z^{2}}\right]+(V(r, \theta, z)-E)\right\}=0
$$

como o potencial não depende de $\theta$, então

$$
\left\{-\frac{\hbar^{2}}{2 \mu}\left[\frac{1}{R(r)}\left[\frac{1}{r} \frac{d R(r)}{d r}+\frac{d^{2} R(r)}{d r^{2}}\right]-\frac{m^{2}}{r^{2}}+\frac{1}{\varphi(z)} \frac{d^{2} \varphi(z)}{d z^{2}}\right]+(V(r, z)-E)\right\}=0
$$

com isso pode-se rearranjar a Eq.(3.116), em uma equação diferencial radial

$$
\left\{-\frac{\hbar^{2}}{2 \mu}\left[\frac{1}{R(r)}\left[\frac{1}{r} \frac{d R(r)}{d r}+\frac{d^{2} R(r)}{d r^{2}}\right]-\frac{m^{2}}{r^{2}}\right]+(V(r)-E)\right\}=0
$$




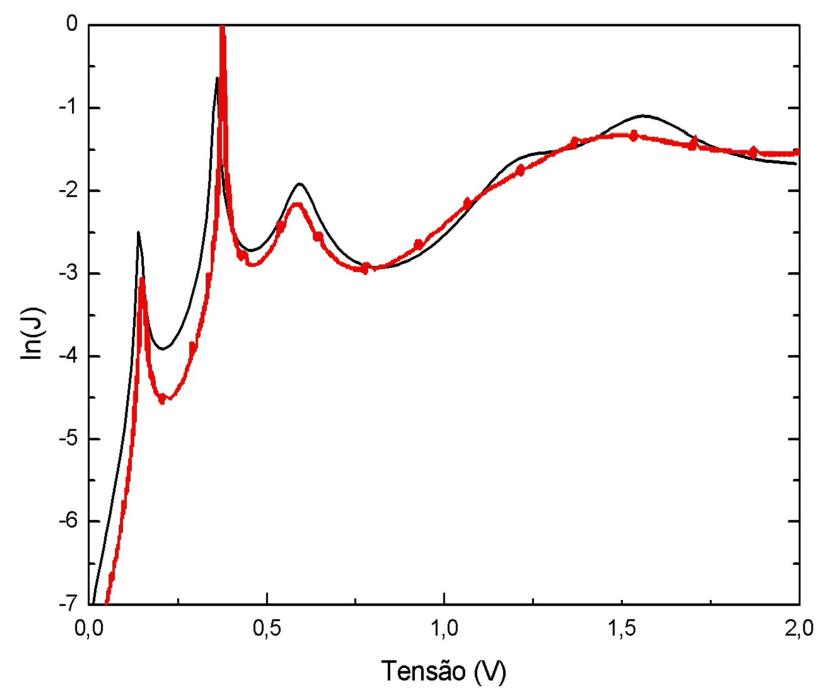

Figura 3.27: Densidade de corrente de tunelamento em função da tensão aplicada para a heteroestrutura semicondutora de três barreiras, com a largura da barreira de $20 \AA$ e altura 0,5 $e V$ e a largura do poço de $50 \AA$, no qual considerou-se a energia incidente do elétron com o valor de $0,005 \mathrm{eV}$. A linha preta representa o resultado obtido neste trabalho e a linha vermelha representa o trabalho proposto por Forrest e Meijer na Ref. [56].

reescrevendo

$$
\left\{-\frac{\hbar^{2}}{2 \mu}\left[\frac{d^{2} R(r)}{d r^{2}}+\frac{1}{r} \frac{d R(r)}{d r}-\frac{m^{2}}{r^{2}} R(r)\right]\right\}=(E-V(r)) R(r)
$$

A solução da parte radial da função de onda será:

$$
R(r)=\mathbf{A} J_{m}\left(\frac{\chi_{n_{r} m}}{r_{0}} r\right)
$$

onde A é um fator de normalização dado por [80],

$$
\mathbf{A}=\left[-\frac{r_{0}^{2} J_{m-1}\left(\chi_{n_{r} m}\right) J_{m+1}\left(\chi_{n_{r} m}\right)}{2}\right]^{-1 / 2}
$$

$J_{m}\left(\frac{\chi_{n_{r} m}}{r_{0}} r\right)$ é a função de Bessel de ordem inteira e $\chi_{n_{r} m}$ - raízes da função de Bessel $\left(n_{r}-\right.$ número quântico radial fixando o número da raiz da função de Bessel como $m$ ).

Portanto, a função de onda ficará da seguinte forma

$$
\Psi(r, \theta, z)=\frac{\mathbf{A}}{\sqrt{2 \pi}} J_{m}\left(\frac{\chi_{n_{r} m}}{r_{0}} r\right) \varphi(z) e^{i m \theta}
$$



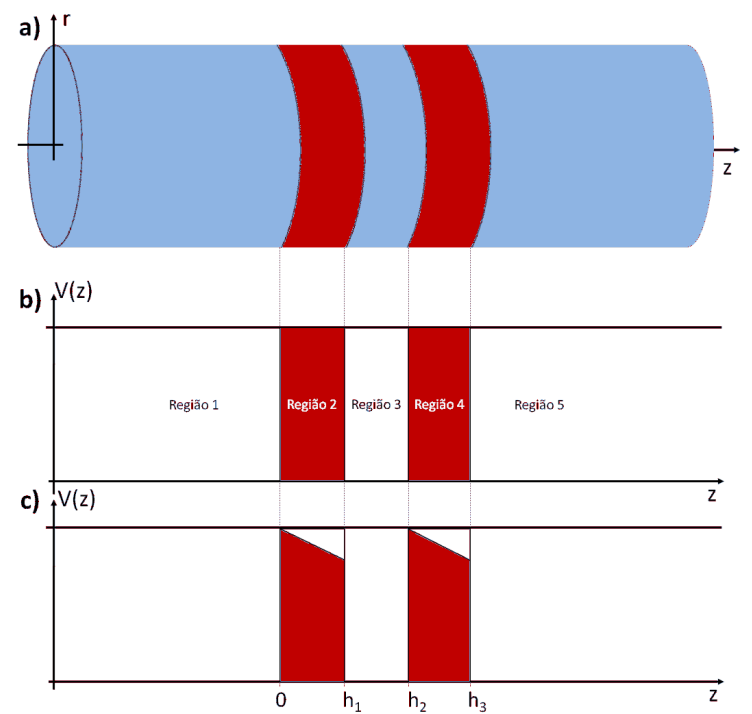

Figura 3.28: a) Representação esquemática do diodo de tunelamento ressonante de fio quântico. b) Representação da dupla barreira de potencial formada a partir das heteroestruturas inseridas no diodo de fio quântico, em função da distância para os casos: sem polarização aplicada e c) com polarização aplicada.

Substituindo a função de onda (3.121) na Eq.(3.116), tem-se a equação para z

$$
\left\{-\frac{\hbar^{2}}{2 \mu}\left[\frac{1}{r} \frac{\partial}{\partial r}\left(r \frac{\partial}{\partial r}\right)+\frac{1}{r^{2}} \frac{\partial^{2}}{\partial \theta^{2}}+\frac{\partial^{2}}{\partial z^{2}}\right]+(V(r, z)-E)\right\} \frac{\mathbf{A}}{\sqrt{2 \pi}} J_{m}\left(\frac{\chi_{n_{r} m}}{r_{0}} r\right) \varphi(z) e^{i m \theta}=0
$$

após algumas manipulações algébricas

$$
\left\{-\frac{\hbar^{2}}{2 \mu}\left[-\frac{\left(\left(\frac{\chi_{n_{r} m}}{r_{0}}\right)^{2} r^{2}-m^{2}\right)}{r^{2}}-\frac{m^{2}}{r^{2}}+\frac{d^{2} \varphi(z)}{d z^{2}} \frac{1}{\varphi(z)}\right]+(V(r, z)-E)\right\}=0
$$

rearranjando

$$
\begin{gathered}
\left\{-\frac{\hbar^{2}}{2 \mu}\left[\frac{d^{2} \varphi(z)}{d z^{2}} \frac{1}{\varphi(z)}-\frac{\left(\frac{\chi_{n_{r} m}}{r_{0}}\right)^{2} r^{2}}{r^{2}}+\frac{m^{2}}{r^{2}}-\frac{m^{2}}{r^{2}}\right]+(V(r, z)-E)\right\}=0 \rightarrow \\
\rightarrow\left\{-\frac{\hbar^{2}}{2 \mu}\left[\frac{d^{2} \varphi(z)}{d z^{2}} \frac{1}{\varphi(z)}-\left(\frac{\chi_{n_{r} m}}{r_{0}}\right)^{2}\right]+(V(r, z)-E)\right\}=0 \rightarrow
\end{gathered}
$$




$$
\rightarrow\left\{-\frac{\hbar^{2}}{2 \mu}\left[\frac{d^{2} \varphi(z)}{d z^{2}}-\frac{\chi_{n_{r} m}^{2}}{r_{0}^{2}} \varphi(z)\right]+(V(r, z)-E) \varphi(z)\right\}=0
$$

Finalmente tem-se a equação para a direção $z$

$$
\frac{d^{2} \varphi(z)}{d z^{2}}+\left(\frac{2 \mu}{\hbar^{2}}(E-V(r, z))-\frac{\chi_{n_{r} m}^{2}}{r_{0}^{2}}\right) \varphi(z)=0
$$

A solução da Eq.(3.123) com as seguintes notações

$$
\begin{aligned}
& k_{0}=\sqrt{\frac{2 \mu_{0}}{\hbar^{2}} E-\frac{\chi_{n_{r} m}^{2}}{r_{0}^{2}}} \\
& k_{1}=\sqrt{\frac{2 \mu_{1}}{\hbar^{2}}\left(V_{0}-E\right)+\frac{\chi_{n_{r} m}^{2}}{r_{0}^{2}}}
\end{aligned}
$$

\subsubsection{Cálculo do $\mathrm{T}(\mathrm{E})$ para o diodo de tunelamento ressonante}

O Método da Matriz Transferência precisou ser adaptado, devido a mudança de simetria, pois no desenvolvimento do MMT na Seção (3.2.1) assumimos as coordenadas cartesianas e nos fios quânticos cilíndricos a simetria agora é cilíndrica. A correção do MMT ocorre somente na substituição da Eq.(3.78) pela (3.125). O MMT é um formalismo versátil. Ele é utilizado para calcular o coeficiente de transmissão para múltiplas barreiras com e sem polarização, e agora pode ser extendido também para fios quânticos cilíndricos.

As funções de onda para as regiões mostradas na Fig.(3.28-b), para o diodo de tunelamento ressonante sem polarização aplicada

$$
\varphi(z)=\left\{\begin{array}{lc}
A_{1} \exp \left(i k_{0} z\right)+A_{2} \exp \left(-i k_{0} z\right), & z<0 \\
B_{1} \exp \left(k_{1} z\right)+B_{2} \exp \left(-k_{1} z\right), & 0<z<h_{1} \\
C_{1} \exp \left(i k_{0} z\right)+C_{2} \exp \left(-i k_{0} z\right), & h_{1}<z<h_{2} \\
D_{1} \exp \left(k_{1} z\right)+D_{2} \exp \left(-k_{1} z\right), & h_{2}<z<h_{3} \\
E_{1} \exp \left(i k_{0} z\right)+E_{2} \exp \left(-i k_{0} z\right), & z>h 3
\end{array}\right.
$$

onde as constantes $A, B, C, D, E$, serão determinadas através das condições de contorno e por meio do método da matriz de transferência (MMT), através da Eq.(3.95), para encontrarmos o coeficiente de transmissão $\mathbf{T}(E)$, como na Eq.(3.99).

As funções de onda para as regiões mostradas na Fig.(3.28-c), para o diodo de tunelamento ressonante com polarização aplicada 


$$
\varphi(z)= \begin{cases}A_{1} \exp \left(i k_{0} z\right)+A_{2} \exp \left(-i k_{0} z\right), & z<0 \\ B_{1} A i\left[-\xi\left(z+z_{B}\right)\right]+B_{2} B i\left[-\xi\left(z+z_{B}\right)\right], & 0<z<h_{1} \\ C_{1} \exp \left(i k_{0} z\right)+C_{2} \exp \left(-i k_{0} z\right), & h_{1}<z<h_{2} \\ D_{1} A i\left[-\xi\left(z+z_{B}\right)\right]+D_{2} B i\left[-\xi\left(z+z_{B}\right)\right], & h_{2}<z<h_{3} \\ E_{1} \exp \left(i k_{0} z\right)+E_{2} \exp \left(-i k_{0} z\right), & z>h 3\end{cases}
$$

onde $A i(z), B i(z)$ são as funções de Airy de primeiro e segundo tipo e

$$
\xi=\left(\frac{2 e F \mu}{\hbar^{2}}\right)^{1 / 3}, \quad z_{B}=\frac{E}{e F}
$$

no qual $F$ é o campo elétrico aplicado. Os cálculos envolvendo polarização de heteroestruturas foi discutido na Seção (3.2), e justificamos a utilização do Método da Matriz Transferência com a aproximação denominada "Step Approximation", principalmente pelo fato de diminuir o esforço computacional. Com isso utilizou-se o formalismo desenvolvido na Seção (3.2), representada pela Eq.(3.104). O coeficiente de transmissão eletrônico de heteroestruturas semicondutoras de fios quânticos cilíndricos com polarização aplicada será

$$
\mathbf{T}(E, V)=\left|\frac{1}{R_{1,1}}\right|^{2}
$$

com a matriz $\mathbf{R}_{j}$ sendo representada pela Eq.(3.95).

De posse da Eq.(3.117), pode-se calcular as energias do sistema através das raízes da função de Bessel, [81],

$$
-\frac{\hbar^{2}}{2 \mu}\left(\frac{d^{2}}{d r^{2}}+\frac{1}{r} \frac{d}{d r}-\frac{m^{2}}{r^{2}}\right) R(r)=E_{\perp} R(r), \quad r \epsilon\left[0, r_{0}\right]
$$

para

$$
R\left(r_{0}\right)=0 \rightarrow E_{\perp n}^{(m)}=\frac{\hbar^{2}}{2 \mu}\left(\frac{\chi_{n_{r} m}}{r_{0}}\right)^{2}
$$

$n=1,2, \ldots$ onde $\chi_{n_{r} m}$ é a $n_{r}$-ésima raiz da função de Bessel $J_{m}(x)$.

Após os cálculos do coeficiente de transmissão eletrônico de um fio quântico cilíndrico, Eq.(3.127), e de suas respectivas energias $E_{\perp n}^{(m)}$, Eq.(3.129), pode-se aplicar tais resultados para obter a dependência do coeficiente de transmissão de um diodo de fio quântico de tunelamento 


\begin{tabular}{|c|c|c|}
\hline Diâmetro $(\mathrm{nm})$ & $\mathrm{R}(\mathrm{nm})$ & $E_{\perp(n=1)}^{(m=0)}$ \\
\hline 30 & 15 & 42,63 \\
\hline 40 & 20 & 23,98 \\
\hline 70 & 35 & 7,83 \\
\hline 100 & 50 & 3,83 \\
\hline
\end{tabular}

Tabela 3.2: Tunelamento eletrônico em função do raio R do fio quântico de InAs com barreiras de InP.

ressonante InAs/InP, Ref. [31] em função do raio do fio quântico, pois o raio do fio quântico afeta diretamente as propriedades eletrônicas, influenciando consequentemente o transporte eletrônico. Um estudo do tunelamento eletrônico em função do raio $R$ do fio quântico de $\operatorname{InAs}$ com barreiras de InP, empregando-se as Eqs.(3.127) e (3.125) foi realizado. Os raios consi derados neste estudo estão mostrados na tabela (3.2), e os resultados correspondentes podem ser analisados através da Fig.(3.29). Nesta figura, vê-se a posição dos picos se alterarem com o raio da estrutura considerada. Entretanto, para raios superiores a $35 \mathrm{~nm}$ não observamos mais uma variação significativa na posição dos picos, ou seja, tais estruturas de fios quânticos comportam-se como estruturas volumétricas tipo "bulk". No trabalho experimental de Björk et al., Ref. [82], tem-se a construção de diodos de fios quântico com tunelamento ressonante, onde os raios dos fios quânticos construídos estão de fato entre 20 a $25 \mathrm{~nm}$. Tudo isto posto, pode-se concluir que, o estudo dos coeficientes de transmissão eletrônico dos diodos de fios quânticos com tunelamento ressonante aqui apresentados estão de acordo com os resultados encontrados na literatura, evidenciando a bem sucedida utilização do Método da Matriz Transferência para problemas desta natureza.

Um estudo incluindo espalhamento em heteroestruturas cilíndricas de tunelamento ressonante através de $n$ barreiras, já vem sendo conduzida por esse autor, a fim de se contrastar com os resultados experimentais de curvas I-V disponíveis na literatura [31]. No entanto, a validação desses cálculos encontram-se ainda em fase de desenvolvimento. 


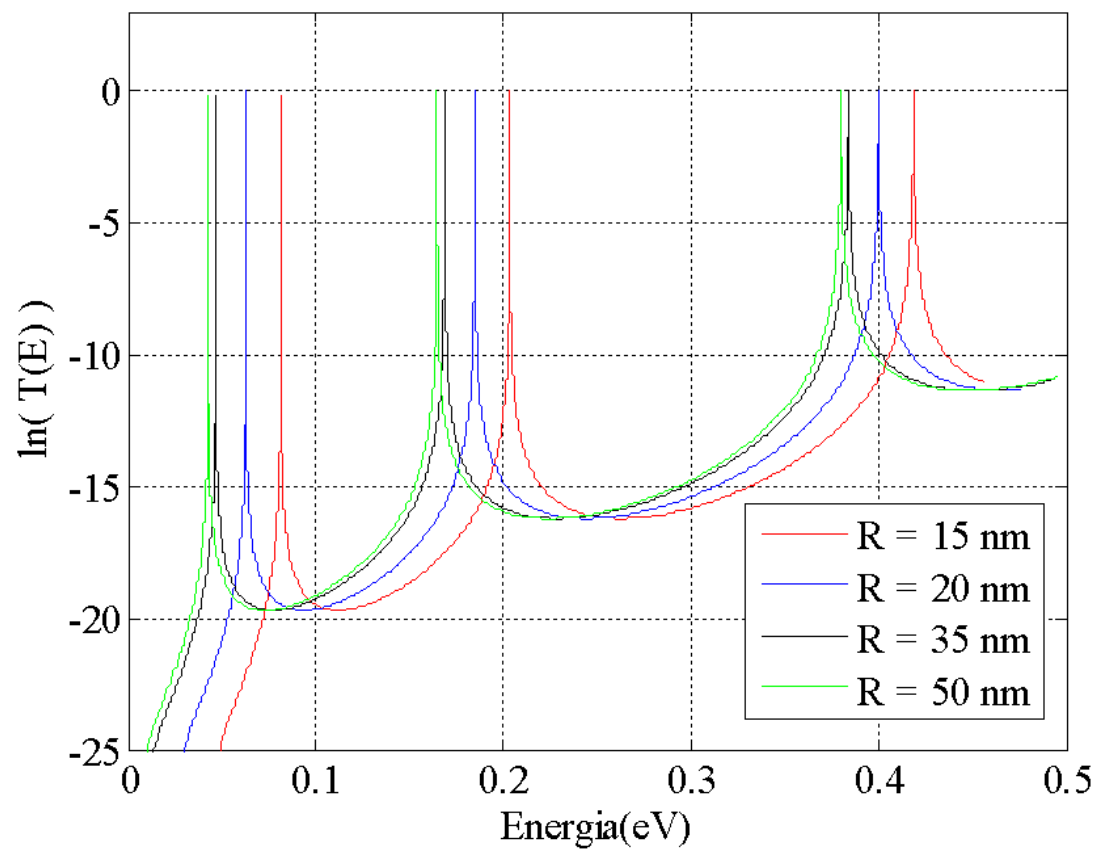

Figura 3.29: Coeficiente de transmissão eletrônico do diodo de fio quântico com tunelamento ressonante em função do raio do fio quântico. 


\section{Capítulo 4}

\section{Conclusão}

O foco desse trabalho foi a modelagem numérica e analítica de dispositivos de heteroestruturas semicondutoras, empregando fios quânticos, visando a obtenção de suas características elétricas.

Inicialmente desenvolveu-se um estudo numérico baseado em busca da solução autoconsistente das equações de Schrödinger-Poisson para a modelagem computacional de heteroestruturas semicondutoras que compreendem um gás de elétrons unidimensional (1-DEG) formado a partir do confinamento eletrostático de um gás eletrônico bidimensional (2-DEG). Tais estruturas são freqüentemente produzidas usando-se técnicas "top-down", a partir de técnicas de litografia e corrossão em estruturas do tipo "split-gate". Os resultados obtidos usando-se o formalismo numérico desenvolvido neste trabalho mostraram concordância bastante satisfatória com aqueles encontrados na literatura. Adicionalmente, ressalta-se que ferramenta numérica aqui apresentada pode ser aplicada a várias outras geometrias de interesse, bastando somente ajustar adequadamente as condições de contorno do sistema escolhido.

A partir de uma revisão da literatura, notou-se, paralelamente, um desenvolvimento experimental crescente e bem sucedido, em inúmeros laboratórios de pesquisa espalhados pelo planeta, permitindo a produção de nanoestruturas cilíndricas semicondutoras de diâmetros da ordem de $10 \sim 100 \mathrm{~nm}$, e comprimentos que podem chegar na ordem de microns, seja na abordagem "top-down" seja na "bottom-up".

Muitos dos fios quânticos assim fabricados, incorporam ainda estruturas de tunelamento ressonante, encontrando um grande potencial para aplicações em dispositivos. Sendo assim, em uma etapa subsequente do nosso trabalho, foi realizado o estudo analítico de heteroestruturas semicondutoras cilíndricas de tunelamento ressonante com n barreiras de poten- 
cial, através do Método da Matriz Transferência. Nossos resultados foram bem sucedidamente contrastados com inúmeros resultados encontrados na literatura.

Finalmente, utilizando-se o método analítico desenvolvido para dispositivos convencionais, com algumas adaptações para sistemas com simetria cilíndrica, calculou-se o coeficiente de transmissão eletrônico em um diodo de fio quântico, para fios de vários diâmetros diferentes. Como atividades futuras, em um programa de doutoramento, um estudo incluindo espalhamento em heteroestruturas cilíndricas de tunelamento ressonante, já vem sendo desenvolvido por esse autor, a fim de se contrastar com os resultados experimentais de curvas I-V disponíveis na literatura. No entanto, a validação desses cálculos encontram-se ainda em fase de desenvolvimento. Além disso, espera-se, em estágios posteriores, estender os cálculos numéricos desenvolvidos neste trabalho para modelar transistores de fios quânticos cilíndricos. 


\section{Referências Bibliográficas}

[1] L. Esaki, "New phenomenon in narrow germanium p-n junctions", Phys. Rev., 109, 2, pp. 603-604, (1958).

[2] J. P. Sun, G. I. Haddad, P. Mazumder, J. N. Schulman, "Resonant tunneling diodes: Models and properties", Proceedings of the IEEE, 86, 4, pp. 641-661, (1998).

[3] J.-S. Wu, C.-Y. Chang, C.-P. Lee, K.-H. Chang, D.-G. Liu, D.-C. Liou, "Characterization of improved AlGaAs/GaAs resonant tunneling heterostructure bipolar transistors", Jpn. J. Appl. Phys., 30, 2A, pp. L160-L162, (1991).

[4] C. Thelander, L.E. Fröberg, C. Rehnstedt, L. Samuelson, L.-E. Wernersson, "Vertical Enhancement-Mode InAs Nanowire Field-Effect Transistor With 50-nm Wrap Gate", IEEE Electron Device Letters, 29, 3, pp. 206-208, (2008).

[5] L. Esaki e R. Tsu, "Superlattice and Negative Differential Conductivity in Semiconductors", IBM Journal of Research and Development, 14, 1, pp. 61-65, (1970).

[6] P. Roblin e H. Rohdin, "High Speed Heterostructure Devices: From device concepts to circuit modeling", Cambridge University Press, Cambridge, UK, (2002).

[7] J. S. Moon, R. Rajavel, S. Bui, D. Wong, D. H. Chow, "Room-temperature InAlAs/InGaAs/InP planar resonant tunneling-coupled transistor", Appl. Phys. Lett., 87, 183110, (2005).

[8] H. Sakaki, "Scattering suppression and high-mobility effect of size-quantized electrons in ultrafine semiconductor wire structures", Jpn. J. Appl. Phys., 19, 12, pp. L735-L738, (1980).

[9] S. K. Islam e F. C. Jain, "Analysis of quantum wire high electron mobility transistor (HEMT) structure", Solid-State Electronics, 39, 4, pp. 615-620, (1996). 
[10] J. Sone, "Electron transport in quantum wires and its device applications", Semicond. Sci. Technol., 7, 3B, pp. B210-214, (1992).

[11] S. Y. Chou e Y. Wang, "Planar double gate quantum wire transistor", Appl. Phys. Lett. 63, 6, pp. 788-790, (1993).

[12] M. Haselman e S. Hauck, "The future of integrated circuits: A survey of nanoelectronics", Proceedings of the IEEE, 98, 1, pp. 11-38, (2010).

[13] G. L. Snider, I. H. Tan, E. L. Hu, "Electron states in mesa-etched one-dimensional quantum well wires", J. Apply. Phys., 68, 6, pp. 2849-2853, (1990).

[14] A. Scherer, M. L Roukes, H. G. Craighead, R. M. Ruthen, E. D. Beebe, J. P. Harbison, "Ultranarrow conducting channels defined in GaAs-AlGaAs by low-energy ion damage", Apply. Phys. Lett., 51, 25, pp. 2133-2135, (1987).

[15] A.V. Borzdov, Pozdnyakov DV, Borzdov VM, "Monte Carlo simulation of nonstationary electron transport in GaAs/AlAs quantum wire", 17th International Crimean Conference on Microwave and Telecommunication Technology, Conference Proceedings, 1 e 2, pp 595-597, (2007).

[16] A. V. Borzdov, D. V. Pozdnyakov, "Scattering of electrons in the GaAs/AlAs transistor structure", Phys. Sol. State, 49, 5, pp 963-967, (2007).

[17] J. B. Khurgin, D. Yang, "Resonant tunneling field-effect transistor based on wave function shape modulation in quantum wires", J. Appl. Phys., 85, 6, pp. 3218-3221, (1999).

[18] C. H. Yang, Y. C. Kao, H. D. Shih, "New field-effect resonant tunneling transistor: Observation of oscilattory transconductance", Appl. Phys. Lett., 55, 26, pp. 2742-2744, (1989).

[19] B. Vinter, A. Tardella, "Tunneling transfer field-effect transistor: A negative transconductance device", Appl. Phys. Lett., 50, 7, pp.410-412, (1987).

[20] H. Sakaki, "Velocity-Modulation Transistor (VMT) - A New Field-Effect Transistor Concept ", Jpn. J. Appl. Phys., 21, 6, pp. L381-L383, (1982).

[21] M. Tomizawa, H. Taniyama, A. Yoshii, "Two-dimensional simulation for Resonant Tunneling Transistor", IEEE Trans. Electron. Dev., 41, 6, (1994). 
[22] T. Waho, K. Maezawa, T. Mizutani, "Resonant Tunneling in a Novel Coupled-QuantumWell Base Transistor ", Jpn. J. Appl. Phys., 30, 12A, pp. L2018-L2020, (1991).

[23] W.-C. Liu, H.-J. Pan, W.-C. Wang, S.-C. Feng, K.-W. Lin, K.-H. Yu, L.-W. Laih, "On the Multiple Negative-Differential-Resistance (MNDR) InGaP/GaAs Resonant Tunneling Bipolar Transistors", IEEE Trans. Electron. Dev., 48, 6, pp. 1054-1059, (2001).

[24] W. P. Wang, Y. Hou, N. Li, Z. F. Li, X. S. Chen, W. Lu, W. X. Wang, H. Chen, J. M. Zhou, E. Wu, H. P. Zeng, "Field effect enhanced quantum dot resonant tunneling diode for high dynamic range light detection", Appl. Phys. Lett. 94, 093511, (2009).

[25] S. Lang, L. Worschech, M. Emmerling, M. Strauß, S. Höfling, A. Forchel, "Selective etching of independent contacts in a double quantum-well structure: Quantum-gate transistor", Appl. Phys. Lett., 92, 062101, (2008).

[26] J. A. Simmons, M. A. Blount, J. S. Moon, S. K. Lyo, W. E. Baca, J. R. Wendt, J. L. Reno, M. J. Hafich, "Planar quantum transistor based on 2D-2D tunneling in double quantum well heterostructures", J. Appl. Phys., 84, 10, pp. 5626-5634, (1998).

[27] J. Wensorra, M.I. Lepsa, S. Trellenkamp, J. Moers, K. M. Indlekofer, H Lüthl, "Gatecontrolled quantum collimation in nanocolumn resonant tunneling transistors", Nanotecnology, 20, 46, (2009).

[28] K. Hiruma, T. Katsuyama, K. Ogawa, M. Koguchi, H. Kakibayashi, G. P. Morgan, "Quantum size microcrystals grown using organometallic vapor phase epitaxy", Appl. Phys. Lett. 59, 4, pp. 431-433, (1991).

[29] W. Wang, M. Liu, A. Hsu, "Hybrid nanoelectronics: Future of computer technology", Journal of Computer Science and Technology, 21, 6, pp. 871-886, (2006).

[30] B. J. Ohlsson, M. T. Björk, M. H. Magnusson, K. Deppert, L. Samuelson, L. R. Wallenberg, "Size-, shape-, and position-controlled GaAs nano-whiskers", Appl. Phys. Lett. 79, 20, pp. 3335-3337, (2001).

[31] C. Thelander, M.T. Björk, M.W. Larsson, A.E. Hansen, L.R. Wallenberg, L. Samuelson, "Electron transport in InAs nanowires and heterostructure nanowire devices", Solid State Communications, 131, 9-10, pp. 573-579, (2004). 
[32] M. Meyyappan, M. K. Sunkara, "Inorganic Nanowires: Applications, Properties, and Characterization", CRC Press, Florida, USA, (2009).

[33] M. S. Gudiksen, L. J. Lauhon, J. Wang, D. C. Smith, C. M. Lieber, "Growth of nanowire superlattice structures for nanoscale photonics and electronics", Nature, 415, pp. 617-620, (2002).

[34] L.-E. Wernersson, T. Bryllert, E. Lind. L. Samuelson, "Wrap-gated InAs nanowire FieldEffect Transistor", International Electron Devices Meeting, Washington, DC, USA, (2005).

[35] M. A. Reed, J. N. Randall, R. J. Aggarwal, R. J. Matyi, T. M. Moore, A. E. Wetsel, "Observation of discrete electronic states in a zero-dimensional semiconductor nanostructure", Phys. Rev. Lett., 60, 6, pp. 535-537, (1988).

[36] M. Tewordt, L. Martín-Moreno, V. J. Law, M. J. Kelly, R. Newbury, M. Pepper, D. A. Ritchie, J. E. F. Frost, G. A. C. Jones,"Resonant tunneling in an $A l_{x} G a_{1-x} A s / G a A s$ quantum dot as a function of magnetic field", Phys. Rev. B, 46, 7, pp.3948-3952, (1992).

[37] M. Tewordt, V. J. Law, M. J. Kelly, R. Newbury, M. Pepper, D. C. Peacock, J. E. F. Frost, D.A. Ritchie, G.A.C. Jones, "Direct experimental determination of the tunneling time and transmission probability of electrons through a resonant tunnelling structure", J. Phys.: Condens. Matter, 2, 45, pp. 8969-8975, (1990).

[38] S. Tarucha, Y. Hirayama, T. Saku, T. Kimura, "Resonant tunneling through one- and zerodimensional states constricted by $A l_{x} G a_{1-x} A s / G a A s / A l_{x} G a_{1-x} A s$ heterojunctions and high-resistance regions induced by focused Ga ion-beam implanation", Phys. Rev. B, 41, 8, pp. 5459-5462, (1990).

[39] D.G. Austing, T. Honda, S. Tarucha, "A new design for submicron double-barrier resonant tunnelling transistors", Semicond. Sci. Technol., 11, 3, pp. 388-391, (1996).

[40] M. Griebel, K. M. Indlekofer, A. Förster, H. Lüth, "Transport properties of gated resonant tunneling diodes in the single electron regime", J. Appl. Phys., 84, 12, pp. 6718-6724, (1998).

[41] J. Wensorra, K. M. Indlekofer, M. I. Lepsa, A. Förster, H. Lüth, "Resonant tunneling in nanocolumns improved quantum colimation", Nano Lett., 5, (2005). 
[42] K. Haraguchi, K. Hiruma, T. Katsuyama, K. Tominaga, M. Shirai, T. Shimada, "Selforganized fabrication of planar GaAs nanowhisker arrays", Appl. Phys. Lett., 69, 3, pp. 386-387, (1996).

[43] K. Haraguchi, K. Hiruma, K. Hosomi, M. Shirai, T. Katsuyama, "Growth mechanism of planar-type GaAs nanowhiskers", J. Vac. Sci. Technol. B, 15, 5, pp. 1685-1687, (1997).

[44] L. Samuelson, C. Thelander, M. T. Björk, M. Borgström, K. Deppert, K. A. Dick, A. E. Hansen, T. Martensson, N. Panev, A. I. Persson, W. Seifert, N. Skold, M. W. Larsson, L. R. Wallenberg, "Semiconductor nanowires for OD and 1D physics and applications", Physica E, 25, 2-3, pp. 313-318, (2004).

[45] E. P. A. M. Bakkers, J. A. van Dam, S. De Franceschi, L. P. Kouwenhoven, M. Kaiser, M. Verheijen, H. Wondergem, P. van der Sluis, "Epitaxial growth of InP nanowires on germanium", Nature Mater., 3, pp. 769-773, (2004).

[46] R. Meijers, T. Richter, R. Calarco, T. Stoica, H.-P. Bochem, M. Marso, H. Lüth,"GaNnanowhiskers: MBE-growth conditions and optical properties", J. Cryst. Growth, 289, 1, pp. 381-386, (2006).

[47] H. Tan, G. L. Snider, L. D. Chang, E. L. Hu, "A self-consistent solution of SchödingerPoisson equations using a nonuniform mesh", J. Appl. Phys., 68, 8, pp. 4071-4076, (1990).

[48] K. Brennan, "The Physics of Semiconductors - with applications to optoelectronics devices", Cambridge University Press, Cambridge, UK, (1999).

[49] A. F. J. Levi, "Applied Quantum Mechanics ", Second Edition. New York. Cambridge University Press, (2006).

[50] J. E. Manzoli, M. A. Romero, O. Hipólito, "On the capacitance-voltage modeling of strained quantum-well MODFETs", IEEE Journal of Quantum Electronics, 34, 12, pp. 2314-2320, (1998).

[51] R. Ragi, J. Manzoli, M. A. Romero, "Modeling the $C$ - $V$ Characteristics of Heterodimensional Schottky Contacts", 32o. European Solid-State Device Research Conference, pag. 623, realizada no período de 24-26 de Setembro de 2002, em Florença, Itália. 
[52] R. Ragi, M. A. Romero e B. Nabet, "On the Modelling of the Electronic Characteristics of Heterodimensional Schottky Contacts ", IEEE Transactions on Electron Devices, 52, 2, pp. 170-175, (2005).

[53] R. Tsu e L. Esaki, "Tunneling in a finite superlattice", Apply. Phys. Lett., 22, 11, pp. 562-564, (1973).

[54] M. S. Dresselhaus, "Solid State Physics. Part 1. Transport Properties of Solids", 6.732 Fall, (2001).

[55] S. Vatannia e G. Gildenblat, "Airy's Functions Implementation of the Transfer-Matrix Method for Resonant Tunneling in Variably Spaced Finite Superlattices", IEEE Journal of Quantum Electronics, 32, 6, pp. 1093-1105, (1996).

[56] K. A. Forrest e P. H. E. Meijer, "Tunneling calculations for GaAs-Al(x)Ga(1-x) as graded band-gap sawtooth superlattices", NASA Center: Goddard Space Flight Center, (1991).

[57] G. T. Einevoll, P. C. Hemmer, J. Thomsen, " Operator ordering in effective-mass theory for heterostructures I. Comparison with exact results for superlattices, quantum wells, and localized potentials", Phys. Rev. B, 42, 6 pp. 3485-3496, (1990); G. T. Einevoll, "Operator ordering in effective-mass theory for heteroestructures. II. Strained systems", Phys. Rev. B, 42, 6, pp. 3497-3502, (1990).

[58] L. Hedin, B. I. Lundqvist, "Explicit local exchange-correlation potentials", J. Phys.C: Solid St. Phys., 4, 14, pp. 2064-2083, (1971).

[59] P. Ruden, G. H. Döhler, "Electronic structure of semiconductors with doping superlattices", Phys. Rev. B, 27, 6, pp. 3538-3546, (1983).

[60] S. Adachi, "GaAs, AlAs and $A l_{x} G a_{1-x} A s:$ Material parameters for use in research and device applications", J. Appl., 58, 3, pp. R1-R29, (1985).

[61] B. Ricco e M. Ya. Azbel, "Physics of ressonant tunneling. The one-dimensional doulebarrier case", Phys. Rev. B, 29, 1970, (1984).

[62] O. Pinaud, "Transient simulations of a resonant tunneling diode", J. Apply. Phys. 92, 4, 1987, (2002).

[63] B. G. Streetman, "Solid State Electronic Devices", Prentice Hall, (1995). 
[64] L. Hrivnák, "Determination of $\Gamma$ electron and light hole effective masses in $A l_{x} G a_{1-x} A$ s on the basis of energy gaps, band-gap offsets, and energy levels in $A l_{x} G a_{1-x} A s / G a A s$ quantum wells", Appl. Phys. Lett., 56, 24, (1990).

[65] M. D. Feit, J. A. Fleck Jr., e A. Steiger. "Solution of the Schrodinger equation by a spectral method", J. Comp. Phys., 47, pp. 412-433, (1982); M. D. Feit e J. A. Fleck Jr., "Solution of the Schrödinger equation by a spectral method II. Vibrational energy levels of triatomic molecules", J. Chem. Phys., 78, pp. 301-308, (1983).

[66] M. H. Degani e J. P. Leburton, "Single-electron states and conductance in lateral-surface superlattices", Phys. Rev. 44, pp. 10901-10904, (1991).

[67] J. E. Manzoli, "Dinâmica de Pacotes de Onda em Fios e Poços Quânticos Duplos Assimétricos e Acoplados", Tese de Mestrado, Instituto de Física e Química de São Carlos, USP. (1993).

[68] M. Goano, ACM Transactions on Mathematical Software, " Algorithm 745: computation of the complete and incomplete Fermi-Dirac integral", 21, 3, pg 221 - 232, (1995); J. A. Slinkman, "Method for efficiently determining a fermi-dirac integrals", US Patent Issued, (2000).

[69] B. Vinter, "Subbands and charge control in a two-dimensional electron gas field-effect transistor", Appl. Phys. Lett. 44, 307, (1984).

[70] A. Trellakis, A. T. Galick, A. Pacelli e U. Ravaioli, "Iteration scheme for the solution of the two-dimensional Schrödinger-Poisson equations in quantum structures", J. Appl. Phys., 81, 12, pp.7880, (1997).

[71] F. A. Berezin, "The Schrödinger Equation", Kluwer Academic Publishers, Moscou, (1983).

[72] S. E. Laux, D. J. Frank e F. Stern, "Quasi-one-dimensional electron state in a split-gate GaAs/AlGaAs heterostructure", Surf. Sci. 196, 1-3, pp. 101-106, (1988).

[73] R. Crook, C. G. Smith, M. Y. Simmons e D. A. Ritchie, "One-dimensional probability density observed using scanned gate microscopy", J. Phys.: Condens. Matter, 12, 50, pp. 735-740, (2000). 
[74] S. Pleutin, H. Grabert, G.-L. Ingold e A. Nitzan, " The electrostatic potential profile along a biased molecular wire: A model quantum mechanical calculation", J. Chem. Phys., 118, 8, pp. 3756-3763, (2003).

[75] C. B. Duke, "Tunneling in solids", Academic, New York, USA, p.32, (1969).

[76] J. H. Davies, "The physics of low-dimensional semiconductors: an introduction", Cambridge University Press, Cambridge, UK, (1998).

[77] A.C. Phillips, "Introduction to quantum mechanics", John Wiley \& Sons Ltd., West Sussex, UK, (2003).

[78] M. Abramowitz e I. A. Stegun. "Handbook of Mathematical Functions", Dover Publications Inc., (1968).

[79] M. Abe, H. Hamaguchi, H. Yamamoto, N. Yamada, "Dwell Time in Asymmetrical DoubleBarrier Structures under DC Bias Voltage", Electronics and Communications in Japan, Parte 2, 88, 3, pp. 1-10, (2005).

[80] N.V. Tkach, I.V. Pronishin, A.M. Makhanets, "Spectrum of an electron in a superlattice along a cylindrical quantum wire", Russian Physics Journal, 41, 2, pp. 178-184, (1998).

[81] P. Racec, "Quantum transpor $t$ in c ylindrical nanowire heterostructures", Mathematical aspects of transport in mesoscopic systems, Dublin Institute for Advanced Studies, Dublin 4-7 December 2008.

[82] M. T. Björk, B. J. Ohlsson, C. Thelander, A. I. Persson, K. Deppert, L. R. Wallenberg, e L. Samuelson, "Nanowire resonant tunneling diodes", Appl. Phys. Lett., 81, 23, pp. 4458-4460, (2002).

[83] E. C. de Oliveira e W. A. Rodrigues Jr., "Funções Analíticas e Aplicações", Editora Livraria da Física, São Paulo, (2006).

[84] R. M. Esiberg, R. Resnick, "Física quântica: Átomos, moléculas, sólidos, núcleos e partículas", Campus, Rio de Janeiro, (1979).

[85] D. U. von Rosenberg, "Methods for the numerical solution of partial differential equations Modern Analytic and Computational Methods in Science and Mathematics", Elsevier, New York, (1975). 


\section{Apêndice A}

\section{Métodos de Diferenças Finitas}

\section{Não-Uniforme (MDFNU)}

A resolução das equações de Schrödinger-Poisson Eqs.(2.1 e 2.5) envolvem derivadas de segunda ordem, aqui escritas na forma de diferenças finitas. Isto significa que o domínio espacial tem sua variável representativa, $x$, tratada não mais como contínua mas como discreta. Assim um erro é introduzido no procedimento de cálculo, erro este que é menor quanto menor for o intervalo de discretização, $\Delta x$, Também a variável temporal, $t$, é discretizada em intervalos $\Delta t$. A denominação diferença finita vem do fato das derivadas envolvidas nas equações diferenciais não serem mais consideradas como o limite quando o intervalo de variação de uma função vai a zero, o intervalo agora tem um valor não nulo e da sua escolha depende a precisão dos cálculos.

Para todos os dispositivos expostos, utilizou-se uma discretização não-uniforme do domínio $x,[47]$. Dessa forma, tem-se a redução dos números de pontos em regiões onde é necessária uma "grande precisão"evitando assim um tempo maior de processamento.

Sendo o domínio espacial discretizado como ilustrado no esquema ilustrado na Fig. (A.1), as derivadas segunda num dado ponto $i$ são indicadas a seguir: 


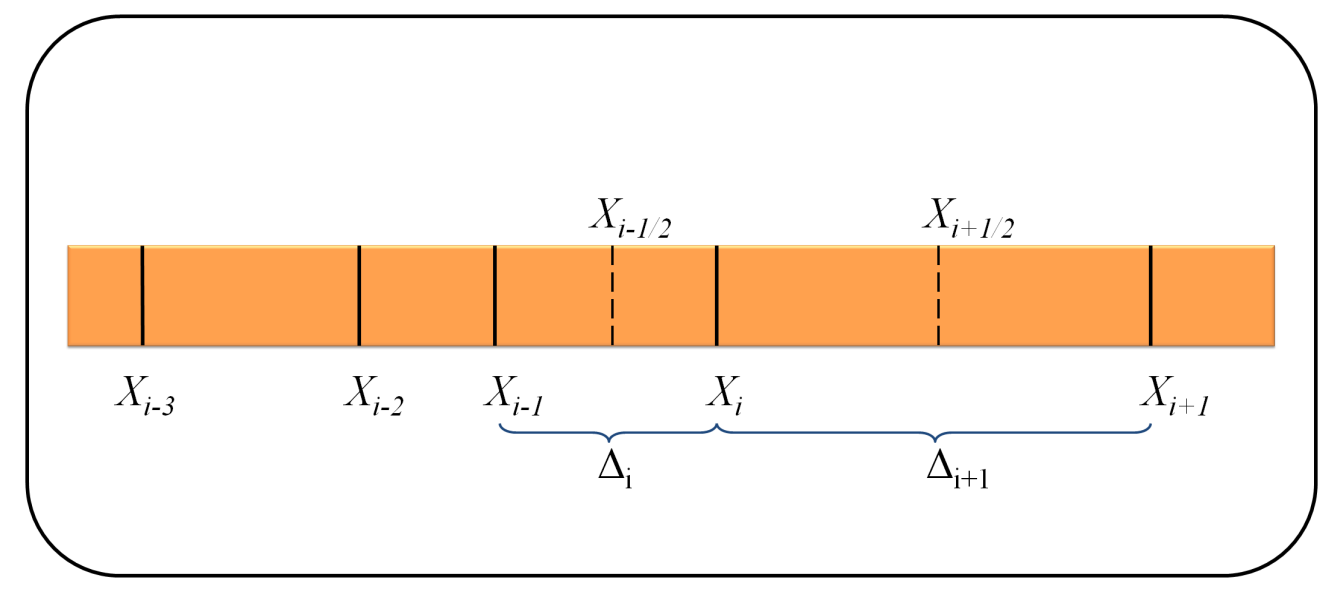

Figura A.1: Representação esquemática da discretização não-uniforme na direção $x$.

$$
\begin{gathered}
\frac{d}{d x} g(x) \frac{d}{d x} f(x) \stackrel{N}{\cong} \frac{d}{d x} g(x)\left[\frac{f_{i+1 / 2}-f_{i-1 / 2}}{\Delta_{i}+\Delta_{i+1}} 2\right]= \\
=\left(g_{i+1 / 2} \frac{f_{i+1}-f_{i}}{\Delta_{i+1}}-g_{i-1 / 2} \frac{f_{i}-f_{i-1}}{\Delta_{i}}\right) \frac{2}{\Delta_{i}+\Delta_{i+1}}= \\
=\left(\frac{2 g_{i+1 / 2}}{\Delta_{i+1}\left(\Delta_{i}+\Delta_{i+1}\right)}\right) f_{i+1}+\left(\frac{2 g_{i+1 / 2}}{\Delta_{i+1}\left(\Delta_{i}+\Delta_{i+1}\right)}+\frac{2 g_{i-1 / 2}}{\Delta_{i}\left(\Delta_{i}+\Delta_{i+1}\right)}\right) f_{i}+\left(\frac{2 g_{i-1 / 2}}{\Delta_{i}\left(\Delta_{i}+\Delta_{i+1}\right)}\right) f_{i-1}
\end{gathered}
$$

Os valores da função $g$ nos pontos intermediários $(i \pm 1 / 2)$ são calculados por interpolação linear.

Temos, então, um conjunto de $N$ expressões da forma descrita na Eq. (A.1), uma para cada ponto de discretização, onde $N$ é o número de pontos em que foi discretizado espacialmente o domínio. Para calcular a derivada num ponto, os 2 pontos vizinhos são utilizados. A expressão para cada ponto é igualada a valores conhecidos e a incógnita a ser determinada é a própria função $f$ em cada ponto (caso da equação diferencial). Este conjunto constitue um sistema linear de equações cuja forma matricial é tridiagonal. Para este trabalho utilizamos o algorítmo de Thomas, [85], em sua resolução. De posse então desse método, podemos agora aplicá-lo a fim de obtermos as soluções auto-consistentes das equações de Schrödinger-Poisson. 


\section{Apêndice B}

\section{Fundamentos de mecânica quântica}

\section{para solução de poços e barreiras de potencial}

Introduzimos inicialmente as noções básicas que serão necessárias para se construir um resultado generalizado para o cálculo do tunelamento através de super-heteroestruturas, envolvendo qualquer número de barreiras e poços quânticos na estrutura, no estado de equilíbrio. O primeiro sistema discutido é aquele aplicado ao problema de condução de elétrons em metais, no qual o sólido é suposto um gás de elétrons livres. Por isso, o modelo que o descreve é conhecido, como modelo da partícula livre. Em seguida são analisados os casos de poços de potencial retangular, infinito e finito, simétricos e assimétricos, triangular, sendo este último muito útil para resolver casos de poços quânticos formados por uma única interface na heteroestruturas semicondutoras com dopagem modulada. Para finalizar, revisitamos a análise de uma única barreira de potencial, adaptamos a análise para os casos de duas e três barreiras de potencial, e a partir desses resultados pode-se generalizar para o caso de qualquer número de poços e barreiras de potencial, na ausência de polarização [48]. Os modelos estudados são de grande interesse para o entendimento de dispositivos que combinam camadas semicondutoras nas mais variadas formas, tendo os casos investigados neste capítulo apresentado resultados satisfatórios quando contrastados com a literatura, [48], [53], [54] e [76]. 


\section{Partícula Livre}

Neste modelo, considere uma partícula de massa $m$, sujeita à um potencial nulo, $V(x)=$ 0. Nestas condições pode-se escrever a Equação de Schrödinger como

$$
H \Psi=E \Psi
$$

$H$ é o hamiltoniano do sistema, escrito em termos do operador energia cinética, $K$, e do operador potencial da partícula, $V$, da forma mostrada abaixo

$$
H=K+V
$$

com

$$
K=\frac{p^{2}}{2 m}
$$

Substituindo a Eq.(B.3) na Eq.(B.2), e assumindo-se o potencial $V$ como uma função da posição $x$, tem-se

$$
H=\frac{p^{2}}{2 m}+V(x)
$$

O operador momento linear $\mathbf{p}$ definido pela mecânica quântica pode ser escrito como

$$
\mathbf{p}=\frac{\hbar}{i} \nabla
$$

assim, a Eq.(B.4) pode ser reescrita como

$$
H=-\frac{\hbar^{2}}{2 m} \nabla^{2}+V(x)
$$

Para problemas unidimensionais e em coordenadas cartesianas,

$$
\nabla^{2}=\frac{\partial^{2}}{\partial x^{2}}
$$

Substituindo a Eq.(B.6) na Eq.(B.5) ficamos com

$$
H=-\frac{\hbar^{2}}{2 m} \frac{\partial^{2}}{\partial x^{2}}+V(x)
$$

A equação de Schrödinger independente do tempo em função da posição $x$ se torna 


$$
\left(-\frac{\hbar^{2}}{2 m} \frac{\partial^{2}}{\partial x^{2}}+V(x)\right) \Psi(x)=E \Psi(x)
$$

ou ainda

$$
-\frac{\hbar^{2}}{2 m} \frac{d^{2} \Psi(x)}{d x^{2}}+V(x) \Psi(x)=E \Psi(x)
$$

Para o caso específico da partícula livre, $V(x)=0$, logo, a Eq.(B.9) pode ser escrita como

$$
-\frac{\hbar^{2}}{2 m} \frac{d^{2} \Psi(x)}{d x^{2}}=E \Psi(x)
$$

e reescrita como

$$
\frac{d^{2} \Psi(x)}{d x^{2}}+\left(\frac{2 m}{\hbar^{2}}\right) E \Psi(x)=0
$$

Fazendo

$$
k=\sqrt{\frac{2 m E}{\hbar^{2}}}
$$

a Eq.(B.11) pode ser reescrita como

$$
\frac{d^{2} \Psi(x)}{d x^{2}}+k^{2} \Psi(x)=0
$$

Logo reconhecemos a Eq.(B.12) como uma equação diferencial de segunda ordem homogênea, cuja solução é uma combinação linear de exponenciais, linearmente independentes:

$$
\Psi(x)=A^{\prime} \exp (i k x)+B^{\prime} \exp (-i k x)
$$

Utilizando a relação de de Moivre [83], teremos:

$$
\Psi(x)=A^{\prime}(\cos (k x)+i \sin (k x))+B^{\prime}(\cos (k x)-i \sin (k x))
$$

Rearranjando, a relação acima:

$$
\Psi(x)=\left(A^{\prime}+B^{\prime}\right) \cos (k x)+\left(i A^{\prime}-i B^{\prime}\right) \sin (k x)
$$

onde $A^{\prime}, B^{\prime}, A$ e $B$ são constantes positivas. Reescrevendo $A=\left(A^{\prime}+B^{\prime}\right)$ e $B=\left(i A^{\prime}-i B^{\prime}\right)$ a função de onda se torna 


$$
\Psi(x)=A \cos (k x)+B \sin (k x)
$$

Sendo assim, a função de onda obtida a partir da Eq.(B.14) descreve o movimento de uma partícula que se move na direção de $x$. Neste caso em particular, o da partícula livre, analisandose a Eq.(B.14) pode-se observar que, as densidades de probabilidade obtidas serão constantes, ou seja, a partícula terá igual probabilidade de ser encontrada em qualquer lugar do espaço, consequentemente, a incerteza da posição será infinita. Tomando-se o princípio da Incerteza de Heisenberg no qual

$$
\Delta p \Delta x \geqslant \frac{\hbar}{2}
$$

sendo $\Delta x=\infty$, concluí-se que $\Delta p \rightarrow 0$, e o momento linear pode ser determinado através da relação [84].

$$
\begin{aligned}
\Delta p & =p-\langle p\rangle=0 \\
p & =\langle p\rangle
\end{aligned}
$$

com $\langle p\rangle$ sendo o valor esperado para o momento, o qual pode ser obtido através da relação

$$
\langle p\rangle=\int_{-\infty}^{\infty} p \Psi^{*}(x) \Psi(x) d x
$$

\section{Poços de Potencial}

Muitas situações de interesse prático como por exemplo as heteroestruturas semicondutoras mais simples podem ser estudadas a partir de modelos ideais da mecânica quântica. Considere a situação exemplificada na Fig.(B.1), que corresponde ao perfil de potencial para uma heteroestrutura semicondutora formada por três regiões diferentes, podendo ser, entre muitas outras combinações possíveis, AlGaAs/GaAs/AlGaAs, formando um poço quântico na região de GaAs, devido a diferença de energia na banda de condução dos diferentes materiais.

Em seguida, apresentamos quatro casos de aproximação que podem ser usadas para descrever situações reais. Entre elas, tem-se o poço de potencial retangular infinito, o poço de potencial retangular finito simétrico, o poço de potencial retangular finito assimétrico e o poço triangular. 


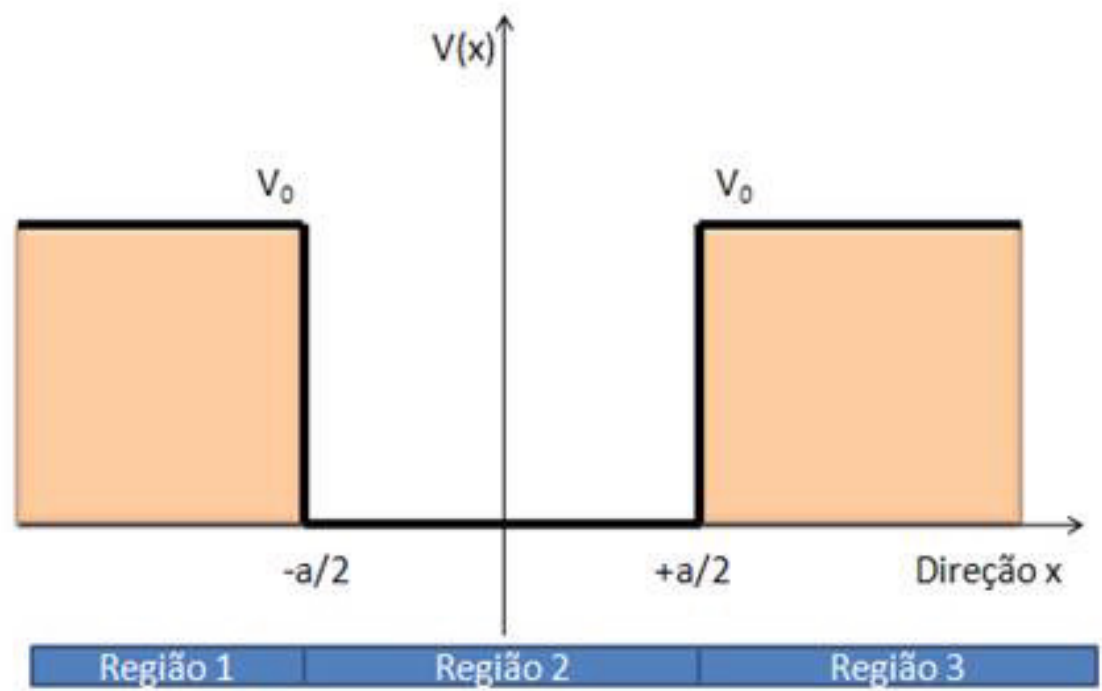

Figura B.1: Representação do poço retangular de potencial finito simétrico de comprimento $a$ e altura $V_{0}$, dividido em três regiões: Região 1,2 e 3 .

Poço de potencial retangular infinito Neste caso, diferentemente do exemplo da partícula livre, confina-se a partícula de massa $m$, dentro de um poço retangular, semelhante àquele mostrado na Fig.(B.1), porém, fazendo-se $V_{o}=\infty$. Por isso, esta aproximação é chamada de poço de potencial infinito, com $V(x)$ satisfazendo a seguinte relação

$$
V(x)=\left\{\begin{array}{lrr}
0, & \text { se } \quad-a / 2 \leq x \leq a / 2 \\
\infty, & \text { se } \quad x<-a / 2 \quad \text { e } \quad x>a / 2
\end{array}\right.
$$

Nas regiões onde o potencial é infinito necessariamente as suas funções de ondas devem ser nulas, ou seja, não existe qualquer probabilidade de encontrar a partícula "fora"do poço. Por outro lado, podemos solucionar a equação de Schrödinger dentro do poço:

Para $-a / 2 \leqslant x \leqslant+a / 2$, temos:

$$
\begin{gathered}
-\frac{\hbar^{2}}{2 m} \frac{d^{2} \Psi_{2}(x)}{d x^{2}}=E \Psi_{2}(x) \rightarrow-\frac{\hbar^{2}}{2 m} \frac{d^{2} \Psi_{2}(x)}{d x^{2}}+E \Psi_{2}(x)=0 \\
\frac{d^{2} \Psi_{2}(x)}{d x^{2}}+k_{2}^{2} \Psi_{2}(x)=0 \quad \text { com } \quad k_{2}=\sqrt{\frac{2 m E}{\hbar^{2}}}
\end{gathered}
$$

Como solução podemos utilizar a Eq.(B.14), 


$$
\Psi_{2}(x)=A \cos \left(k_{2} x\right)+B \sin \left(k_{2} x\right)
$$

Para as regiões $x<-a / 2$ e $x>+a / 2$, teremos respectivamente:

$$
\Psi_{1}(x)=0 \quad \text { e } \quad \Psi_{3}(x)=0
$$

Utilizando as seguintes condições de contorno:

$$
\begin{aligned}
& \Psi_{1}(x=-a / 2)=\Psi_{2}(x=-a / 2) \\
& \Psi_{2}(x=+a / 2)=\Psi_{3}(x=+a / 2)
\end{aligned}
$$

Da Eq.(B.20), obtém-se

$$
0=A \cos \left(k_{2} \frac{a}{2}\right)-B \sin \left(k_{2} \frac{a}{2}\right)
$$

Da Eq.(B.21), obtém-se

$$
0=A \cos \left(k_{2} \frac{a}{2}\right)+B \sin \left(k_{2} \frac{a}{2}\right)
$$

Somando as Eq.(B.22) e Eq.(B.23), tem-se

$$
2 A \cos \left(k_{2} \frac{a}{2}\right)=0
$$

Subtraindo as Eq.(B.22) e Eq.(B.23), tem-se

$$
2 B \sin \left(k_{2} \frac{a}{2}\right)=0
$$

Agora, as Equações (B.24) e (B.25), precisam ser satisfeitas simultaneamente. Assumindo que $A$ e $B$ sejam constantes diferentes de zero

$$
\begin{aligned}
& \cos \left(k_{2} \frac{a}{2}\right)=0 \\
& \sin \left(k_{2} \frac{a}{2}\right)=0
\end{aligned}
$$


Com isso, precisa-se encontrar uma condição que possa resolver as Equações (B.26) e (B.27) simultaneamente

$$
\begin{gathered}
\cos \left(k_{2} \frac{a}{2}\right)=0 \rightarrow k_{2} \frac{a}{2}=\frac{\pi}{2}, \frac{3 \pi}{2}, \frac{5 \pi}{2}, \ldots \rightarrow k_{2}=\frac{n \pi}{a}, n=1,3,5 \ldots \\
\sin \left(k_{2} \frac{a}{2}\right)=0 \rightarrow k_{2} \frac{a}{2}=\pi, 2 \pi, 3 \pi, \ldots \rightarrow k_{2}=\frac{n \pi}{a}, n=2,4,6 \ldots
\end{gathered}
$$

Assim, pode-se substituir os valores de $k_{2}$ na solução (B.18) e levar em conta as condições de contorno do problema

$$
\begin{aligned}
& \Psi_{1}(x)=A_{n} \cos \left(\frac{n \pi}{a} x\right) \quad\left(1^{a} \text { Classe }\right) \\
& \Psi_{2}(x)=B_{n} \sin \left(\frac{n \pi}{a} x\right) \quad\left(2^{a} \text { Classe }\right)
\end{aligned}
$$

Encontrada as soluções, sem determimar as constantes $A_{n}$ e $B_{n}$, que serão encontradas utilizando a condição de normalização

$$
\int_{-\infty}^{+\infty} \Psi^{*}(x) \Psi(x) d x=1
$$

Substituindo a Eq.(B.30) na Eq.(B.32), obtém-se

$$
\int_{-a / 2}^{+a / 2}\left[A_{n} \cos \left(\frac{n \pi}{a} x\right)\right]^{*}\left[A_{n} \cos \left(\frac{n \pi}{a} x\right)\right] d x=1 \rightarrow A_{n}^{2} \int_{-a / 2}^{+a / 2}\left[\cos ^{2}\left(\frac{n \pi}{a} x\right)\right] d x=1
$$

Fazendo uma mudança de variáveis $u=\frac{n \pi}{a} x$

$$
\begin{aligned}
A_{n}^{2}\left(\frac{a}{n \pi}\right) \int_{-n \pi / 2}^{+n \pi / 2}\left[\cos ^{2}(u)\right] d u & =1 \rightarrow A_{n}^{2}\left(\frac{a}{n \pi}\right)\left(\frac{n \pi}{2}\right)=1 \\
\rightarrow A_{n} & =\sqrt{\frac{2}{a}}
\end{aligned}
$$

Substituindo a Eq.(B.31) na Eq.(B.32), obtém-se

$$
\int_{-a / 2}^{+a / 2}\left[B_{n} \sin \left(\frac{n \pi}{a} x\right)\right]^{*}\left[B_{n} \sin \left(\frac{n \pi}{a} x\right)\right] d x=1 \rightarrow B_{n}^{2} \int_{-a / 2}^{+a / 2}\left[\sin ^{2}\left(\frac{n \pi}{a} x\right)\right] d x=1
$$


Fazendo uma mudança de variáveis $w=\frac{n \pi}{a} x$

$$
\begin{aligned}
B_{n}^{2}\left(\frac{a}{n \pi}\right) \int_{-n \pi / 2}^{+n \pi / 2}\left[\sin ^{2}(w)\right] d w & =1 \rightarrow B_{n}^{2}\left(\frac{a}{n \pi}\right)\left(\frac{n \pi}{2}\right)=1 \\
\rightarrow B_{n} & =\sqrt{\frac{2}{a}}
\end{aligned}
$$

Com isso temos as soluções de ambas as classes

$$
\begin{aligned}
& \Psi_{2}(x)=\sqrt{\frac{2}{a}} \cos \left(\frac{n \pi}{a} x\right) \quad\left(1^{a} \text { Classe }\right) \\
& \Psi_{2}(x)=\sqrt{\frac{2}{a}} \sin \left(\frac{n \pi}{a} x\right) \quad\left(2^{a} \text { Classe }\right)
\end{aligned}
$$

Para finalizar o problema precisamos encontrar a expressão para os auto-estados de energia

$$
\begin{array}{ll}
k_{2}^{2}=\left(\frac{n \pi}{a}\right)^{2}=\frac{2 m E_{n}}{\hbar^{2}} \rightarrow E_{n}=\frac{\pi^{2} \hbar^{2}}{2 m a^{2}} n^{2} & n=1,3,5, \ldots \\
k_{2}^{2}=\left(\frac{n \pi}{a}\right)^{2}=\frac{2 m E_{n}}{\hbar^{2}} \rightarrow E_{n}=\frac{\pi^{2} \hbar^{2}}{2 m a^{2}} n^{2} & n=2,4,6, \ldots
\end{array}
$$

Como as Equações (B.35) e (B.36), são equivalentes, pode-se generalizar para todos os $\mathrm{n} \in \mathbb{N}:$

$$
E_{n}=\frac{\pi^{2} \hbar^{2}}{2 m a^{2}} n^{2} \quad \mathrm{n}=1,2,3, \ldots
$$

Por fim, pode-se verificar um diagrama da função de onda $\Psi(x)$, Fig.(B.2) encontrada e sua densidade de probabilidade, $|\Psi(x)|^{2}$, Fig.(B.3) para $n=1,2$ e 3 .

De acordo com o Princípio da Incerteza de Heisenberg, é impossível determinar a posição da partícula dentro do poço devido a sua incerteza conjunta com o momento linear $\hat{p}$. Sendo assim, com o intuito de conhecer as regiões de menor probabilidade de se encontrar o elétron, obteve-se o comportamento da densidade de probabilidade $|\Psi(x)|^{2}$ com função da posição $x$, como ilustrado na Fig.(B.3), para os três primeiros modos, $n=1,2,3$. 


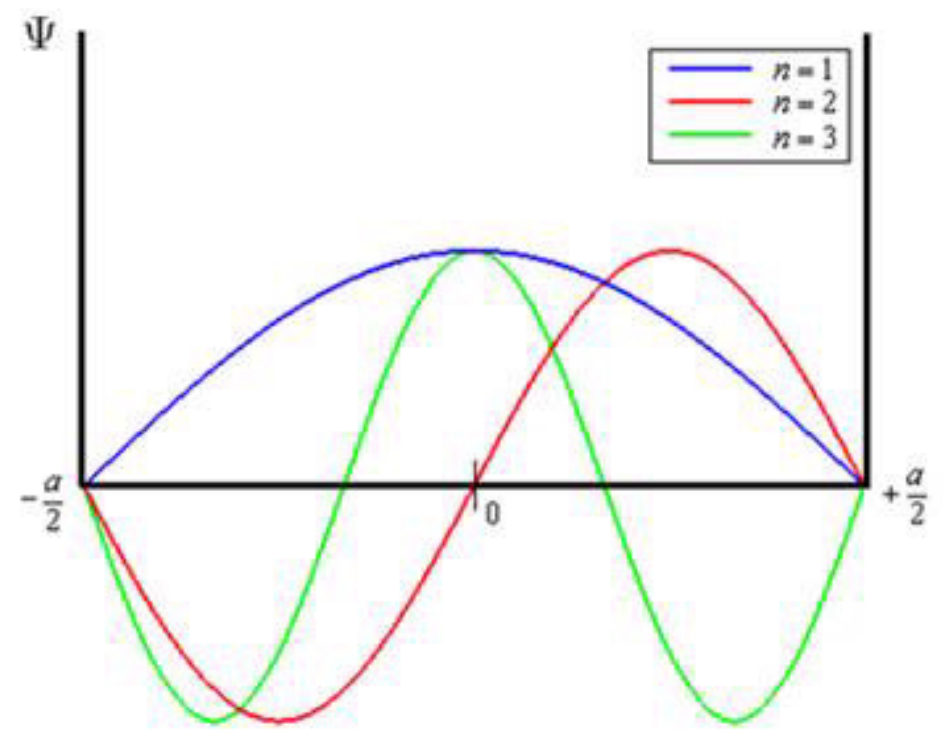

Figura B.2: Representação das funções de ondas $(\Psi(x))$ para $n=1,2$ e 3 .

Poço de potencial retangular finito simétrico $\mathrm{O}$ desenvolvimento de técnicas de fabricação de heteroestruturas semicondutoras, tais como MBE, MOCVD, etc. propiciou a possibilidade de se preparar estruturas que apresentam níveis discretos de energia para elétrons e lacunas como resultado de um confinamento quântico, obtido a partir da deposição camada a camada de semicondutores compostos em camadas adjacentes com diferentes intervalos de banda proibida.

A estrutura de um poço de potencial finito simétrico é representado na Fig.(B.1). A representação mostra a divisão do poço em três regiões denominadas por 1, 2 e 3 . Será necessário resolver a Equação de Schrödinger para as respectivas regiões assim como as condições de contorno nas interfaces. Iremos tratar aqui o caso em que $E<V_{0}$.

A equação de Schrödinger nas regiões 1 e 3, onde o potencial é o mesmo, é:

$$
-\frac{\hbar^{2}}{2 m} \frac{d^{2} \Psi(x)}{d x^{2}}+V_{0} \Psi(x)=E \Psi(x)
$$

onde $\Psi_{1}(x)=A e^{k_{1} x}$ e $\Psi_{3}(x)=B e^{-k_{1} x}$, as constantes $A$ e $B$ são reais e positivas. A constante $k_{1}$ é denominada

$$
k_{1}=\sqrt{\frac{2 m\left(V_{0}-E\right)}{\hbar^{2}}}
$$

A Equação de Schrödinger e a solução da região 2 será 


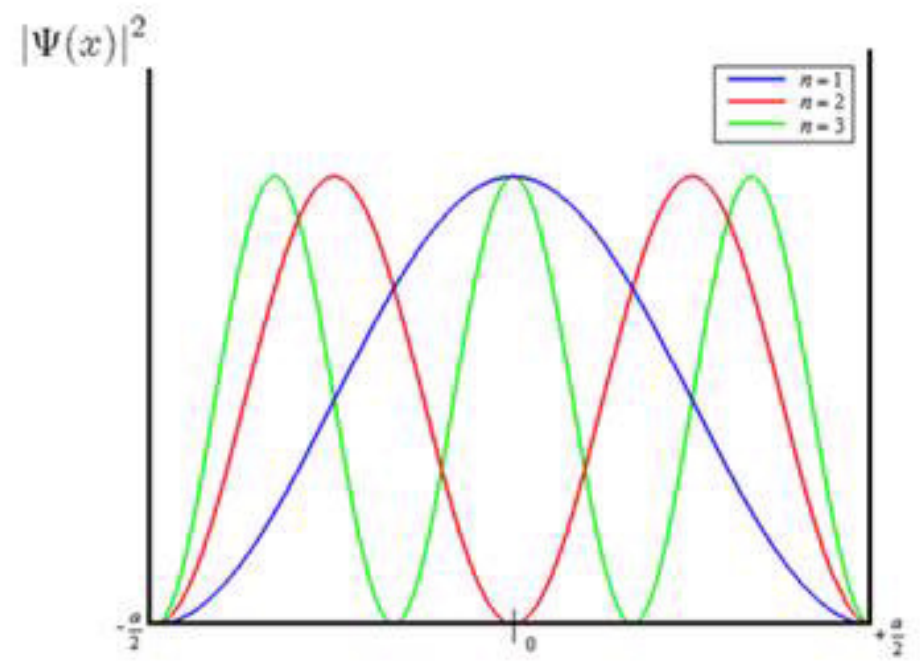

Figura B.3: Representação das densidades de probabilidade $\left(|\Psi(x)|^{2}\right)$ para $n=1,2$ e 3 .

$$
\begin{gathered}
-\frac{\hbar^{2}}{2 m} \frac{d^{2} \Psi(x)}{d x^{2}}=E \Psi(x) \\
\Psi_{2}(x)=C \cos \left(k_{2} x\right)+D \sin \left(k_{2} x\right)
\end{gathered}
$$

com

$$
k_{2}=\sqrt{\frac{2 m E}{\hbar^{2}}}
$$

Aplicando as condições de contorno nas interfaces entre os distintos materiais semicondutores

$$
\begin{aligned}
\Psi_{i}(x) & =\Psi_{j}(x) \\
\frac{1}{m_{i}} \frac{d}{d x} \Psi_{i}(x) & =\frac{1}{m_{j}} \frac{d}{d x} \Psi_{j}(x)
\end{aligned}
$$

Substituindo $x=\frac{-a}{2}$ nas condições de contorno (B.41), tem-se

$$
\begin{gathered}
A e^{\frac{-k_{1} a}{2}}=C \cos \left(\frac{k_{2} a}{2}\right)-D \sin \left(\frac{k_{2} a}{2}\right) \\
k_{1} A e^{\frac{-k_{1} a}{2}}=k_{2} C \cos \left(\frac{k_{2} a}{2}\right)+k_{2} D \sin \left(\frac{k_{2} a}{2}\right)
\end{gathered}
$$


Substituindo $x=\frac{a}{2}$ nas condições de contorno (B.41), tem-se

$$
\begin{gathered}
B e^{\frac{-k_{1} a}{2}}=C \cos \left(\frac{k_{2} a}{2}\right)+D \sin \left(\frac{k_{2} a}{2}\right) \\
-k_{1} B e^{\frac{-k_{1} a}{2}}=-k_{2} C \sin \left(\frac{k_{2} a}{2}\right)+k_{2} D \cos \left(\frac{k_{2} a}{2}\right)
\end{gathered}
$$

Somando as Equações (B.42) e (B.44), obtém-se

$$
(A+B) e^{\frac{-k_{1} a}{2}}=2 C \cos \left(\frac{k_{2} a}{2}\right)
$$

Subtraindo as Equações (B.44) e (B.42), obtém-se

$$
(B-A) e^{\frac{-k_{1} a}{2}}=2 D \sin \left(\frac{k_{2} a}{2}\right)
$$

Somando as Equações (B.43) e (B.45), obtém-se

$$
k_{1}(A-B) e^{\frac{-k_{1} a}{2}}=2 k_{2} D \cos \left(\frac{k_{2} a}{2}\right)
$$

Subtraindo as Equações (B.43) e (B.45), obtém-se

$$
k_{1}(A+B) e^{\frac{-k_{1} a}{2}}=2 k_{2} C \sin \left(\frac{k_{2} a}{2}\right)
$$

Divindo as Equações (B.49) e (B.46) e assumindo que as constantes $A$ e $B$ sejam diferentes de zero, teremos uma equação transcendental, denominada $1^{\circ}$ Tipo:

$$
k_{1}=k_{2} \tan \left(\frac{k_{2} a}{2}\right)
$$

Divindo as Equações (B.48) e (B.47) e assumindo que as constantes $A$ e $B$ sejam diferentes de zero, teremos outra equação transcendental, denominada $2^{o}$ Tipo:

$$
-k_{1}=k_{2} \cot \left(\frac{k_{2} a}{2}\right)
$$

Antes de resolver as equações de $1^{o}$ e $2^{o}$ Tipo, utilizaremos a seguinte mudança de variáveis,

$$
y=\sqrt{\frac{m E a^{2}}{2 \hbar^{2}}}
$$

teremos: 


$$
\begin{aligned}
y \tan y & =\sqrt{\frac{m V_{0} a^{2}}{2 \hbar^{2}}-y^{2}} \\
-y \cot y & =\sqrt{\frac{m V_{0} a^{2}}{2 \hbar^{2}}-y^{2}}
\end{aligned}
$$

Considere um elétron de massa $m=9,11 \times 10^{-31} \mathrm{~kg}$, preso a um poço de potencial de largura $a=10 \AA$ e altura do poço, $V_{0}=0,347 \mathrm{eV}$. Para encontrar a energia do estado fundamental do elétron deve-se resolver as equações transcendentais dadas pelas Eqs.(B.52 e B.53), através de um método gráfico, ou um método numérico, que pode ser bissecção ou NewtonRaphson. A solução gráfica obtida forneceu para $n=1$

$$
E=\frac{2 \hbar^{2} y^{2}}{m a^{2}} \Longrightarrow E_{1}=0,128260 \mathrm{eV}
$$

O método de bissecção utilizado com uma tolerância de $\varepsilon=1 \times 10^{-5}$ fornece o valor da energia do estado fundamental de $E_{1}=0,1283 \mathrm{eV}$, e o método de Newton-Raphson, considerando uma raiz inicial de $x_{i}=0,45$, e uma tolerância de $\varepsilon=1 \times 10^{-5}$, um valor de energia de $E_{1}=0,128305 \mathrm{eV}$.

Vale a pena mencionar que, como era de se esperar, para alturas de barreira, $V_{o}$, cada vez maiores, o resultado fornecido pelo modelo do poço de potencial finito se aproxima dos valores para o poço de potencial infinito.

Aplicação do poço retangular de potencial finito simétrico Neste ponto, vamos mostrar que os resultados obtidos a partir de modelos ideais, simples, da mecânica quântica pode nos fornecer resultados úteis. Considere uma heteroestrutura semicondutora como a indicada na Fig.(B.4). Nas condições em que foi construída a sequência de camadas semicondutoras, a estrutura forma um dispositivo de interessante aplicação, funcionando como um laser de poço quântico. Suponha que desejemos conhecer o comprimento de onda no qual será emitido o laser, e assim determinar a faixa de frequência no espectro eletromagnético. Sendo assim, é possível chegar a esse valor se for calculado a energia $E_{p h}$ com a qual um fóton é emitido durante a recombinação elétron-lacuna na estrutura de poço quântico, que pode ser obtida se for determinado os níveis de energia dos elétrons e lacunas no poço quântico, como mostrado na seção anterior. A soma dessas energias com a energia de banda proibida do material, $E_{G}$, fornece a energia com que o fóton é emitido: 


$$
E_{p h}=E_{1 \text { condução }}+E_{1 \text { valência }}+E_{G}
$$

Considere um poço retângular de $G a A s / A l_{0,45} G a_{0,55} A s$ com largura de $a=25 \AA$, para o qual deseja-se calcular para os níveis $n=1$ dos elétrons e das lacunas neste poço. Assumindo a discontinuidade na banda de condução de $0,345 \mathrm{eV}$ e a descontinuidade na banda de valência de 0,213eV , é possível conhecer os níveis de energias de forma análoga ao discutido anteriormente. Seguindo-se então aqueles mesmos passos chega-se a $E_{1}=0,037271 \mathrm{eV}$ para a energia dos elétrons na banda de condução e $E_{1}=0,033122 \mathrm{eV}$ para a energia das lacunas na banda de valência, ambos através do método gráfico.

Sendo a banda proibida do $\mathrm{GaAs}$ de $1,42 \mathrm{eV}$, portanto a energia do fóton emitido, é simplesmente

$$
E_{p h}=0,037271+0,033122+1,42=1,490393 e V
$$

Sabendo que a energia se relaciona com o comprimento de onda da seguinte forma

$$
E_{p h}=\frac{\mathrm{h} c}{\lambda} \rightarrow \lambda=\frac{\mathrm{h} c}{E_{p h}}
$$

onde h é a constante de Planck e $c$ é a velocidade da luz no vácuo.

Desta forma, um laser de semicondutor com as características acima emite uma luz com comprimento de onda igual a $\boldsymbol{\lambda}=\mathbf{8 3 4 . 6 6} \mathbf{n m}$, que corresponde a uma emissão na faixa do infravermelho.

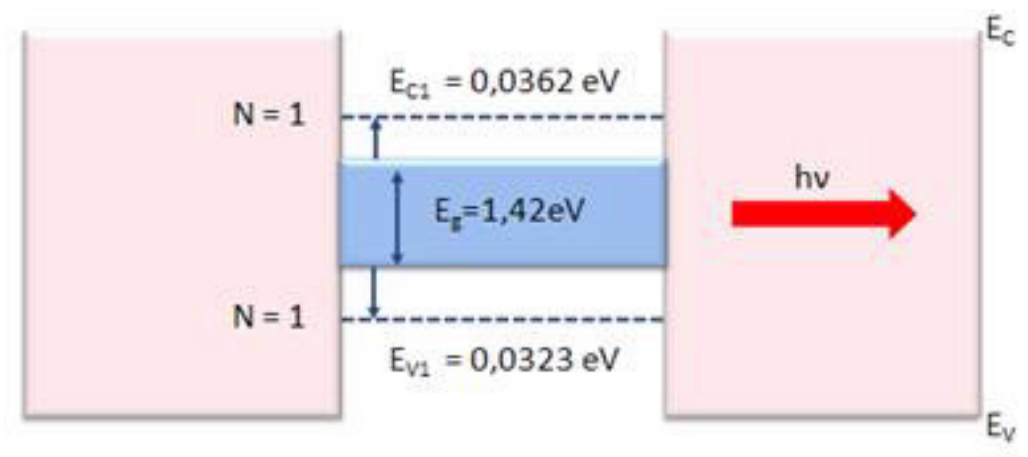

Figura B.4: Esquema das bandas de energia para um sistema de heteroestrutura semicondutora mostrando os efeitos da quantização espacial e a energia de um fóton emitido após uma recombinação elétron-lacuna. 
Poço de potencial retangular finito assimétrico Neste exemplo, iremos encontrar a solução para o poço de potencial finito assimétrico Fig.(B.5), assumindo também que a massa da partícula seja a mesma nas três regiões. Para resolvermos o problema é necessário resolver a Equação de Schrödinger para cada uma das três regiões. Posteriormente utilizar as condições de contorno nas intersecções das regiões 1,2 e 3 e o potencial $V_{1}$ para a região $1, V_{2}$ igual a zero na região 2 e $V_{3}$ para a região 3 .

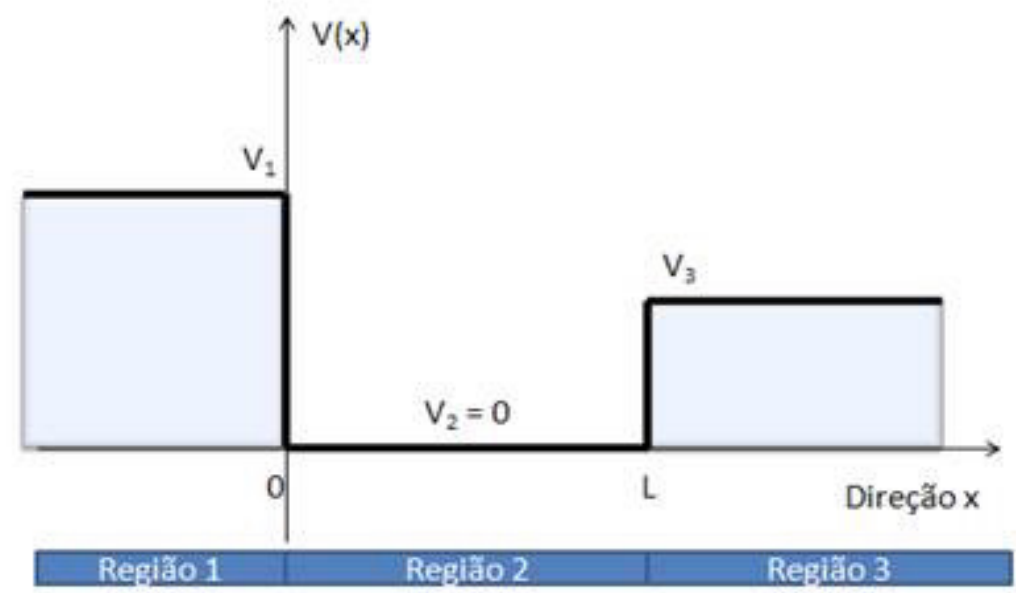

Figura B.5: Poço quadrado de potencial finito assimétrico de comprimento L, dividido em três regiões: Região 1,2 e 3.

Escrevendo agora a solução da equação de Schrodinger para as três regiões tem-se

\section{Região 1:}

$$
\Psi_{1}(x)=A e^{k_{1} x} \quad \text { com } \quad k_{1}=\sqrt{\frac{2 m\left(V_{1}-E\right)}{\hbar^{2}}}
$$

Região 2:

$$
\Psi_{2}(x)=A \cos k x+B \sin k x \quad \text { com } \quad k=\sqrt{\frac{2 m E}{\hbar^{2}}}
$$

\section{Região 3:}

$$
\Psi_{3}(x)=(A \cos k L+B \sin k L) e^{-k_{3}(x-L)} \quad \operatorname{com} \quad k_{3}=\sqrt{\frac{2 m\left(V_{3}-E\right)}{\hbar^{2}}}
$$

Utilizando agora as condições de contorno, em $x=0$ : 


$$
\begin{aligned}
A-A & =0 \\
k_{1} A-A k & =0
\end{aligned}
$$

$\operatorname{Em} x=L$, temos:

$$
\begin{aligned}
A \cos k L+B \sin k L-(A \cos k L+B \sin k L) & =0 \\
A\left(k_{3} \cos k L-k \sin k L\right)+B\left(k_{3} \sin k L+k \cos k L\right) & =0
\end{aligned}
$$

Montando uma equação matricial, tem-se

$$
\left[\begin{array}{l}
A \\
B
\end{array}\right]\left[\begin{array}{cc}
k_{1} & -k \\
k_{3} \cos k L-k \sin k L & k_{3} \sin k L+k \cos k L
\end{array}\right]=\left[\begin{array}{l}
0 \\
0
\end{array}\right]
$$

Como $A$ e $B$ são diferentes de zero, tem-se

$$
\operatorname{det}=\left[\begin{array}{cc}
k_{1} & -k \\
k_{3} \cos k L-k \sin k L & k_{3} \sin k L+k \cos k L
\end{array}\right]=0
$$

Efetuando o determinante

$$
\sin k L\left(k_{1} k_{3}-k^{2}\right)+\cos k L\left(k_{1} k+k k_{3}\right)=0 \Longrightarrow \tan k L=\frac{k_{1} k+k k_{3}}{k^{2}-k_{1} k_{3}}
$$

Multiplicando ambos os lados por $L^{2}$ e rearranjando

$$
\tan k L=\frac{\left(k_{1} L+k_{3} L\right) k L}{k^{2} L^{2}-k_{1} L k_{3} L}
$$

Manipulando as expressões de $k_{1}, k$ e $k_{3}$

$$
\begin{aligned}
& k_{1}=\sqrt{\frac{2 m\left(V_{1}-E\right)}{\hbar^{2}}}=\sqrt{\frac{2 m V_{1}}{\hbar^{2}}-\frac{2 m E}{\hbar^{2}}}=\sqrt{Q-k^{2}} \\
& k_{3}=\sqrt{\frac{2 m\left(V_{3}-E\right)}{\hbar^{2}}}=\sqrt{\frac{2 m V_{3}}{\hbar^{2}}-\frac{2 m E}{\hbar^{2}}}=\sqrt{Q^{6}-k^{2}}
\end{aligned}
$$

fazendo a seguinte mudança de variável, $x=k L$ e substituindo na Eq.(B.63), tem-se 


$$
\tan x=\frac{x\left(L \sqrt{Q-\frac{x^{2}}{L^{2}}}+L \sqrt{Q^{6}-\frac{x^{2}}{L^{2}}}\right)}{x^{2}-L^{2}\left(\sqrt{Q-\frac{x^{2}}{L^{2}}} \sqrt{Q^{6}-\frac{x^{2}}{L^{2}}}\right)}
$$

Definindo as seguinte funções:

$$
\begin{aligned}
& f(x)=\tan x \\
& g(x)=\frac{x\left(L \sqrt{Q-\frac{x^{2}}{L^{2}}}+L \sqrt{Q^{6}-\frac{x^{2}}{L^{2}}}\right)}{x^{2}-L^{2}\left(\sqrt{Q-\frac{x^{2}}{L^{2}}} \sqrt{Q^{6}-\frac{x^{2}}{L^{2}}}\right)}
\end{aligned}
$$

Com isso encontramos mais uma equação transcendental que não apresenta uma solução analítica e sim uma solução numérica, que será apresentada a seguir.

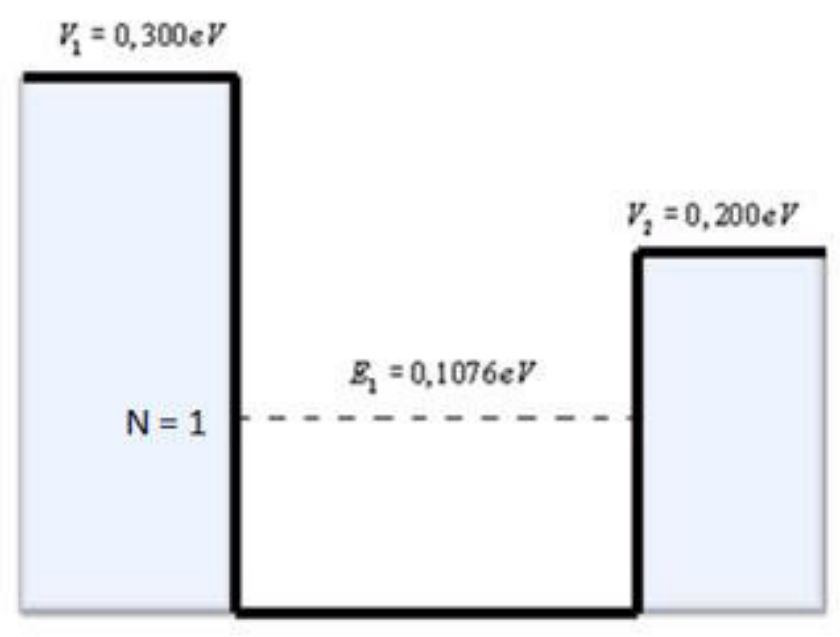

Figura B.6: Esquema do estado acessível do poço de potencial finito assimétrico.

Considere um poço de potencial assimétrico como mostrado na Fig.(B.5), no qual, $L=10 \AA, V_{1}=0,200 \mathrm{eV}$ e $V_{3}=0,300 \mathrm{eV}$. Procedendo-se de forma análoga ao realizado para o caso do poço de potencial retangular finito simétrico, utilizando-se o método gráfico, obtém-se

$$
x=k L \Longrightarrow\left(\frac{x}{L}\right)^{2}=\frac{2 m E}{\hbar^{2}} \Longrightarrow E_{1}=\frac{x^{2} \hbar^{2}}{2 m L^{2}} \Longrightarrow E_{1}=0,1076 \mathrm{eV}
$$

Poço de potencial triangular O poço triangular é uma aproximação do perfil de potencial produzido devido a dopagem modulada de heterointerfaces semicondutoras, conforme ilustrado 
pela Fig.(B.7). Há uma barreira infinta em $z<0$ (Região 1) com um potencial linear $V(z)=e F z$ para $z>0$ (Região 2). De acordo com a Lei de Gauss, $V(z)$ é descrito pela carga $e$ e o campo elétrico $F$ (assumindo que o produto $e F$ será positivo). Note que $F$ é usado para descrever o campo elétrico para não haver confusão com a energia $E$ da Equação de Schrödinger [76].

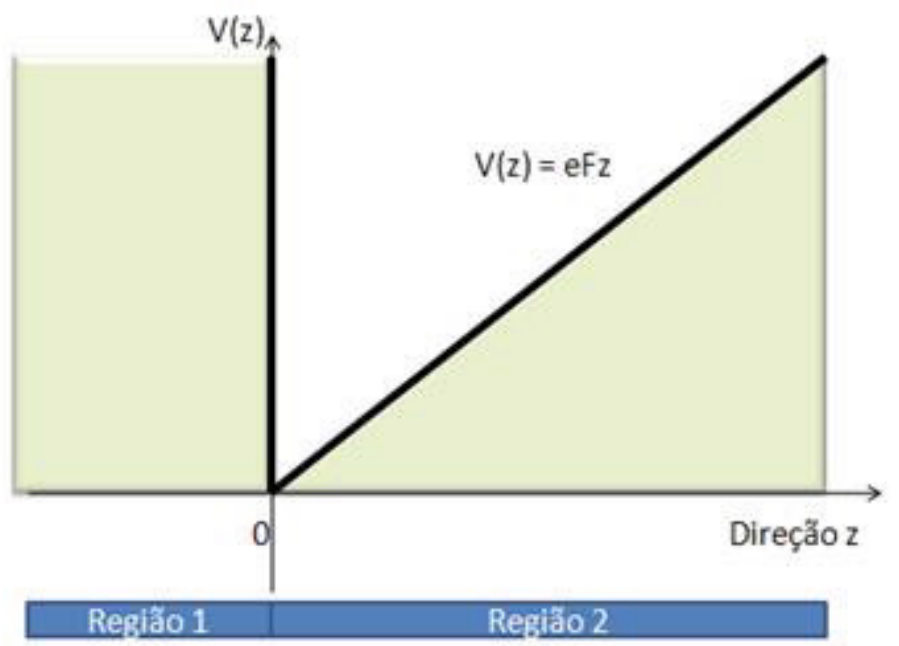

Figura B.7: Representação do poço de potencial triangular, dividido em duas regiões: Região 1 e 2 .

Precisamos resolver a Equação de Schrödinger

$$
\left[-\frac{\hbar^{2}}{2 m} \frac{d^{2}}{d z^{2}}+e F z\right] \Psi(z)=E \Psi(z)
$$

onde a condição de contorno $\Psi(z=0)=0$, é devido a barreira infinita. Agora iremos introduzir uma mudança de variáveis.

$$
z_{0}=\left(\frac{\hbar^{2}}{2 m e F}\right)^{1 / 3}, \quad E_{0}=\left[\frac{(e F \hbar)^{2}}{2 m}\right]=e F z_{0}
$$

A Equação de Schrödinger será

$$
\frac{d^{2} \Psi}{d \bar{z}^{2}}=(\bar{z}-\bar{E}) \Psi(z)
$$

assim nós podemos definir uma nova mudança de variavel $x=\bar{z}-\bar{E}$, a Eq.(B.71) se reduz a Equação de Airy

$$
\frac{d^{2} \Psi}{d x^{2}}=x \Psi
$$


As funções de Airy provém da solução da Equação de Schrödinger com um potencial linear, de suma importância para o 2-DEG em heteroestruturas. A Eq.(B.72) é uma equação diferencial de segunda ordem que tem duas soluções que são linearmente independentes. Estas soluções serão as Funções Integrais de Airy $A i(x)$ e $B i(x)$.

As duas soluções independentes, as funções de $A i(x)$ e $B i(x)$,

$$
\Psi(x)=C A i(x)+D B i(x)
$$

onde $C$ e $D$ são constantes.

Precisamos analisar quando $z \rightarrow+\infty$, ou seja $x \rightarrow+\infty$. A solução escolhida tal que $A i(x) \rightarrow 0$ com $x \rightarrow+\infty$, entretanto $B i(x)$ diverge.

$$
\Psi(x)=C A i(x)
$$

para $|x|$ grande, temos as expansões assintóticas das funções de Airy, no qual Ai(x) será

$$
\begin{gathered}
A i(x) \sim \pi^{-1 / 2} x^{-1 / 4} \exp \left(-\frac{2}{3} x^{3 / 2}\right) \\
A i(-x) \sim \frac{1}{2} \pi^{-1 / 2} x^{-1 / 4} \cos \left(\frac{2}{3} x^{3 / 2}-\frac{1}{4} \pi\right)
\end{gathered}
$$

A função de onda não-normalizada será

$$
\Psi(x) \simeq A i(x) \simeq A i(\bar{z}-\bar{E}) \simeq A i\left(\frac{e F z-E}{E_{0}}\right)
$$

condição de normalização:

$$
\begin{aligned}
& \int_{-\infty}^{+\infty} \Psi^{*}(x) \Psi(x) d x=1 \rightarrow \int_{0}^{L_{W}} C^{2}[A i(x)]^{2} d x=1 \\
& C^{2} \int_{0}^{L_{W}}\left[\pi^{-1 / 2} x^{-1 / 4} \exp \left(-\frac{2}{3} x^{3 / 2}\right)\right]^{2} d x=1 \rightarrow
\end{aligned}
$$

Para encontrarmos o valor de $C$, precisamos utilizar o computador para resolver esta integral, como isso não é de suma importância, ignoramos o seu cálculo.

Temos $V(x)=e F x$ para $x>0$ e uma parede em $x=0$. As energias dependem dos zeros de $A i(x)$, que pode ser encontrada numericamente, por uma simples aproximação 


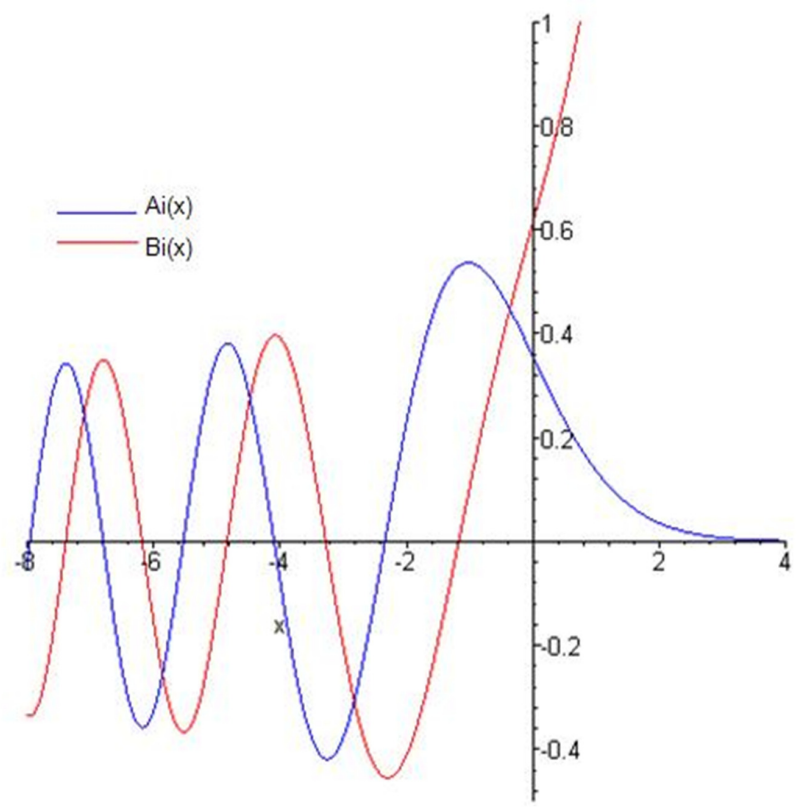

Figura B.8: Comportamento das duas funções de Airy: $A i(x)$ e $B i(x)$.

analítica. Considerando as condições de contorno do lado esquerdo sendo $x_{L}=0$ e a do lado direito $x_{R}=E / e F$. A condição de quantização é

$$
\begin{aligned}
\left(n-\frac{1}{4}\right) \pi & =\int_{x_{L}}^{x_{R}} k(x) d x=\int_{0}^{E / e F}\left[\frac{2 m}{\hbar^{2}}(E-e F x)\right]^{1 / 2} d x \\
& =\left[\frac{2 m E_{n}}{\hbar^{2}}\right]^{1 / 2} \frac{E_{n}}{e F} \int_{0}^{1} \sqrt{1-s} d s
\end{aligned}
$$

onde $s=x / x_{R}$. A integral será

$$
\begin{gathered}
\int_{0}^{1} \sqrt{1-s} d s=\frac{2}{3} \\
\rightarrow\left(n-\frac{1}{4}\right) \pi=\left[\frac{2 m E_{n}}{\hbar^{2}}\right]^{1 / 2} \frac{E_{n}}{e F} \frac{2}{3}
\end{gathered}
$$

Logo $E_{n}$ será

$$
E_{n}=\left[\frac{3}{2} \pi\left(n-\frac{1}{4}\right)\right]^{2 / 3}\left[\frac{(e F \mathrm{~h})^{2}}{2 m}\right]^{1 / 3}
$$


Então, temos os auto-estados de energia do poço de potencial triangular para $n=$ $1,2,3, \ldots$, onde o $E_{n}$ é energia associada à n-ésima banda. 